\title{
VARIATION, TRANSMISSION, AND \\ MAINTENANCE OF TRADITIONAL ECOLOGICAL \\ KNOWLEDGE ON MALEKULA ISLAND, \\ VANUATU
}

By

Joe McCarter

A thesis

submitted to the Victoria University of Wellington

in fulfilment of the requirements for the degree of

Doctor of Philosophy

in Environmental Studies

Victoria University of Wellington 


\section{Abstract}

The traditional ecological knowledge (TEK) of local and indigenous people supports the resilience of social-ecological systems and is an important aspect of global biocultural diversity. However, TEK is at risk of erosion amid social and ecological change, and may be threatened by homogenising influences such as formal school systems. Loss of TEK is of particular concern in countries such as Vanuatu, a culturally diverse nation where TEK is a critical component of resource management, medicinal practice, and adaptive capacity. This thesis aims to fill four key literature gaps surrounding the variation, transmission, and maintenance of TEK, and is situated in four rural communities on Malekula Island, Vanuatu.

This research employs an interdisciplinary mixed-methods approach across four core chapters. The first uses multi-model inferencing to show that systems of TEK are dynamic and complex, and that change is variable across domains of knowledge and segments of the population. Data in this chapter indicate that ethnomedical knowledge is at risk of erosion in younger age cohorts. The second core chapter shows that the cultural transmission of TEK is predominantly vertical in nature (i.e. passed from parent to child), is initiated during the early teens, and that some skills (such as the carving of ceremonial items) appear to be less salient to younger age cohorts. This chapter also notes a changing developmental niche for TEK transmission, and suggests that changes in both the setting and strategies for knowledge transmission are key mechanisms that drive TEK variation. The third and fourth core chapters discuss aspects of TEK maintenance: first, by examining top-down strategies for TEK revitalisation by eliciting local perceptions of the value of TEK to formal school systems; and second, by identifying the triggers and barriers to bottom-up, in situ TEK maintenance in local organisations known as 'kastom schools'. Both approaches have potential to conserve aspects of TEK, however in situ approaches may be best for encouraging the maintenance of knowledge and practice within an appropriate cultural context. There are, however, significant epistemological barriers to both top-down and bottom-up approaches to TEK maintenance, which may ultimately limit their effectiveness.

This research fills several key literature gaps and employs a diverse and novel range of analytical tools. The data presented here highlight the depth, diversity and importance of TEK in Vanuatu, and emphasise the need for careful and considered approaches to its maintenance. However, these findings also show the dynamic and complex nature of cultural change, and suggest that attempts at TEK measurement and maintenance must be cognisant of temporal and spatial variation in the 
drivers and impact of TEK change. Moreover, this research emphasises that TEK systems are inextricably bound in wider issues of power, heterogeneity, and political ecology, and that TEK maintenance is fundamentally linked to self-determination and the lived value of tradition in contemporary social contexts.

\section{Keywords}

Traditional ecological knowledge, cultural change, ethnobotanical knowledge, multi-model inferencing, diversity indices, cultural transmission, developmental niche, formal education, contextualised education, cultural conservation, cultural revitalisation, in situ cultural conservation, linguistic ecology, Vanuatu, Malekula Island, Malakula Island, Oceania, mixed-methods research. 


\section{Samari}

Long fulap ples long world, kastom save blong envaeroment (KSBE) hemi stap lus. Ol jenis ia bambae i mekem i hard blong ol man blong lukaotem ol risos blong hem, mo bambae i mekem i hard blong ol jeneresen long fiuja blong stap strong taem envaeromen i jenis. Vanuatu hemi wan ples we i gat fulap difren kalja mo difren kaen envaeromen, mo kastom mo tredisen oli gat fulap importance long laef long ol man. I gat fulap rison we from wanem KSBE hemi important long Vanuatu: sam example olsem saed long lukaotem ol risos, saed long givim meresin long ol man, mo saed long givhan long ol man blong stap strong taem envaeromen i jenis. Be long Vanuatu, i gat wan problem, from we KSBE mo kastom i save lus, from we fulap niufala samting i kam insaed, ol envaeromen i jenis, mo ol youngfala oli no lanem gud ol save blong ol bubu blong olgeta.

Risej ia i stap givhan blong saed long lanem save hao nao KSBE i stap lus, mo hao nao yumi save lukaotem hem mo mekem se i stap oltaem. Mi bin work long fofala komuniti long Malekula Island, mo mi bin traem save trifala samting. Namba wan samting, mi stap traem luk sipos KSBE hemi stap lus, mo wanem kaen samting i stap mekem se KSBE i lus. Mi bin yusum wan kaen interviu wetem 177 man mo woman long West Coast Malekula, mo mi askem evri wan blong telemaot fulap samting, olsem ol lif blong meresin. Ol risal oli telem se ol youngfala oli gat fulap KSBE, be olgeta oli no gat save olsem ol oldfala. Ol oldfala nomo oli gat save blong medel bus, be ol youngfala oli no stap lanem ol samting ia. Save ia i stap lus bigwan wetem ol man we oli go long market fulap mo we oli no laekem blong toktok lanwis tumas. Namba tu samting, mi stap traem luk hao nao ol man mo woman oli stap lanem ol save blong olgeta. Mi faendem se fulap long ol man mo woman oli lanem long mama mo papa blong olgeta, be mi luk save se ol fasin blong tijim ol difren kaen KSBE hemi stap jenis. Namba tri samting, mi stap luk long saed long hao nao yumi save lukaotem ol KSBE mo mekem se i stap ol taem. Mi bin storian wetem fulap man mo woman long saed long wanem oli stap tingting nao saed long lukaotem KSBE. Mi bin faendem se i gat sam kaen KSBE we yumi save putum i go long skul, olsem save blong mekem garen. Be i gat fulap samting we yumi no save putum insaed skul, minim se yumi mas faendem wan nara fasin blong lukaotem KSBE. Ol 'kastom skul' blong Malekula bambae oli gud smol: oli gat fulap problem, be oli save lukaotem fulap difren kaen kastom we yumi no save putum insaed skul.

Risej ia hemi soemaot importance blong KSBE long Vanuatu, mo hemi telem se i important we bambae yumi lukaotem KSBE blong mekem se i no lus. Be risej ia I telem tu se ol jenis we i stap kam long saed long kastom long komuniti, bambae oli hard lelebit blong lukaotem KSBE long evri 
ples blong evri man. Ol fasin blong jenis long KSBE bambae oli jenis evri yia, mo bambae oli difren long evri ples long Malekula mo long Vanuatu. Mo tu, risej ia i telem se ol rison we KSBE i lus hemi fastem taet long ol fasin blong power mo long difrens bitim ol difren ples. Wan ples we samting ia i important hemi saed long skul long vilaj mo komuniti. Blong finisem risej ia, mi mekem sam recommendation blong policy saed long lukaotem KSBE blong Malekula. 


\section{Acknowledgements}

Although only one name appears on the cover, this thesis would not have come close to completion without the input, support, and coaxing of a large number of people.

The first and loudest thanks must go to my primary supervisor at Victoria, Dr Michael Gavin, whose enthusiasm, wisdom, and commitment were always above and beyond the call. Mike, I'm deeply grateful for your guidance and input over the past four years, and am looking forward to continuing this work into the future. My secondary supervisors, Dr Andrew McGregor and Dr Sean Weaver, made insightful contributions at critical times, and I sincerely appreciate the time and effort they've extended on my behalf.

Of course, this thesis would not have happened without the support, patience, and generosity of my friends, hosts, and research associates in Vanuatu. In particular, I'd like to acknowledge the fieldworkers of Malekula, including the curator of the Malekula Cultural Centre Numa Fred Longga, Chief Virambath at Unmet (and his wife Yvette), Chief Joachim Kaibaba (Dixon Reef), Hantison Sangmuru (Tisvel), James Teslo (Lewinevet), and Chief Alben Reuben (Lawa); as well as other friends who helped, hosted, and drank kava with me along the way, including Melton Sinmor (Wigmo), Roy Wariat (Lawa), Chief Tony Mbong (Tisvel), and Eric Sangmuru (Tisvel). The staff at the Vanuatu Cultural Centre in Port Vila were integral in shaping this research, and I'd particularly like to thank Ralph Regenvanu and Sue Baraeleo for their sustained input and guidance. The knowledge of Sam Chanel at the Vanuatu Department of Forestry has been a critical resource to many a researcher in Vanuatu, and I'd like to thank him for the important role it played here. Finally, I'd like to reserve a special mention for Chief Mateas Battick of Lorlow, who passed away in 2009. His patience, generosity, and infectious chuckle form some of the most indelible memories from my first field trip to Malekula, and I've no doubt his warmth and incredible depth of knowledge continue to be greatly missed in South-West Bay and around Vanuatu.

Back in New Zealand, I have been ably assisted in my endeavours by a large cast of characters. Editorial support from Nigel McCarter, Tim Garlick, and Connie Nisbet greatly improved the final product, and was much appreciated. My erstwhile colleagues in the Ministry of Hard Work (Mark, John, Ed, Claire, Aaron, Charlie, Rob, Matilda, Ruifei, Amani, and Mary, amongst others) provided the hilarity, competition, and motivation to keep going, despite mostly absenting themselves a couple of years too early. Other colleagues at Victoria (including Laura, Richie, Meghan, Monica, Abu, Emilio, Jeroen, Jodie and Perrine) proved themselves to be excellent foils for discussion and vents 
for frustration, a task which has also been stoically borne by a gang of mates in Wellington whom I'll thank (and apologise to) in person. The Bell family provided the perfect writer's retreat at Hicks Bay for which I remain extremely grateful. Finally, to Mum, Dad, Tom and Sarah go sincere thanks: the unflappable McCarter brand of kindness and humour has helped me immensely throughout.

I am extremely grateful to the primary funders of this research, the Ryoichi Sasakawa Young Leaders Fellowship program, who supported this work between 2009 and 2011. Additional funding was provided by the JL Stewart Scholarship (2008); the Education New Zealand Study Abroad Award (2008); the NZAID Postgraduate Field Research Award (2008); the Royal Society of New Zealand (2010); and by two grants from the Victoria University Faculty of Science (2008 and 2009). 


\section{Abbreviations}

CBD: Convention on Biological Diversity

CRP: Cultural Research Policy

DN: Developmental Niche framework

HEC: Human Ethics Committee

IPR: Intellectual Property Rights

MCC: Malekula Cultural Centre

NCC: Vanuatu National Council of Chiefs

SIL: Summer Institute of Linguistics

SPC: Secretariat of the Pacific Community

TEK: Traditional ecological knowledge

VCC: Vanuatu Cultural Centre

VUW: Victoria University of Wellington 


\section{Table of Contents}

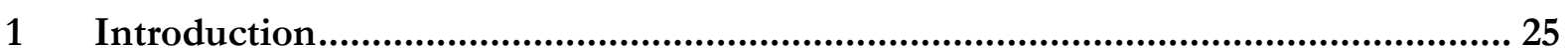

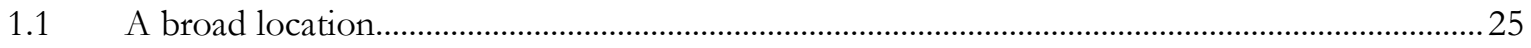

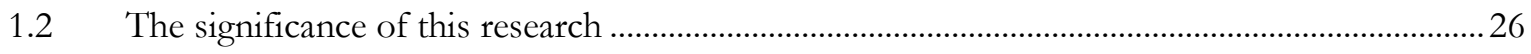

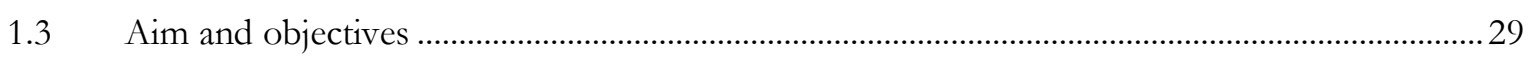

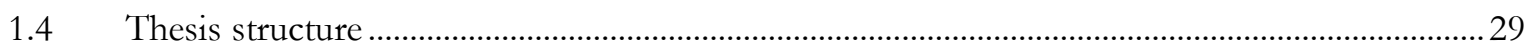

2 TEK: Definition, application, and current trends .................................................31

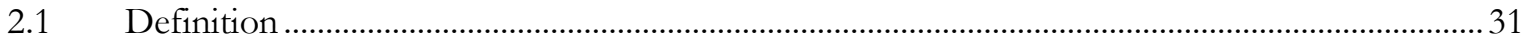

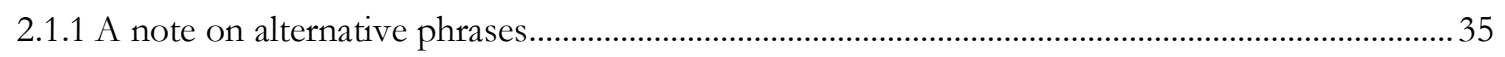

2.2 Why TEK? Values of TEK in contemporary academia ....................................................................

2.3 Controversies and representation in TEK research ................................................................... 40

2.3.1 TEK and 'western' knowledge.................................................................................................... 40

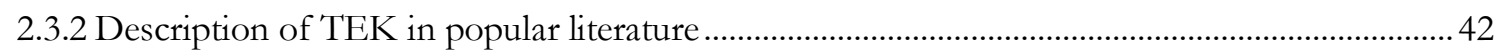

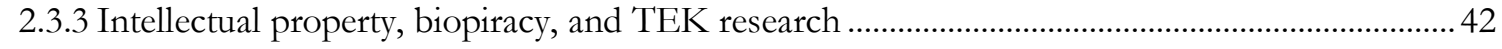

2.4 A resource at risk? TEK erosion and the problems of measurement ........................................... 44

2.4.1 Measuring variation and erosion of TEK …………................................................................... 46

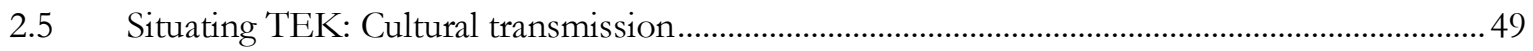

2.6 Revitalising culture: TEK maintenance ................................................................................... 49

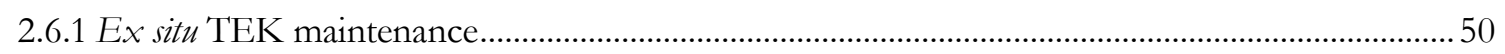

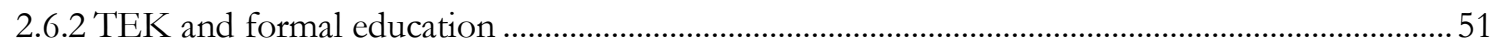

2.6.3 In situ TEK maintenance............................................................................................................ 53

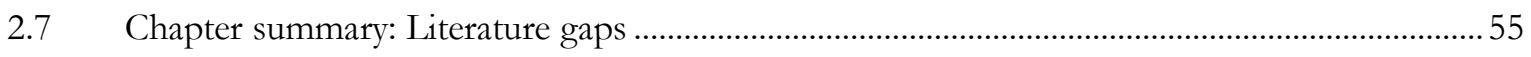

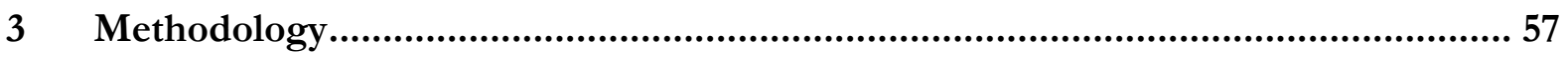

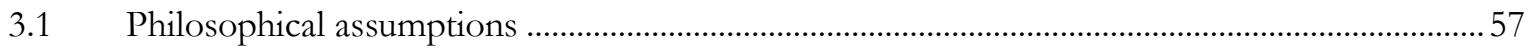

3.1.1 Positionality..................................................................................................................... 57 


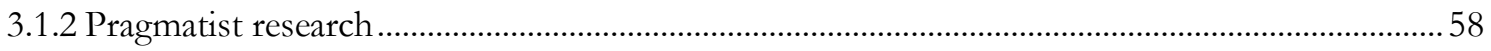

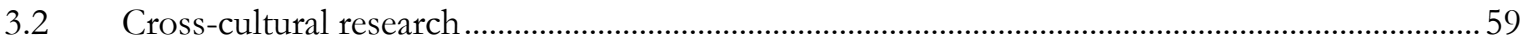

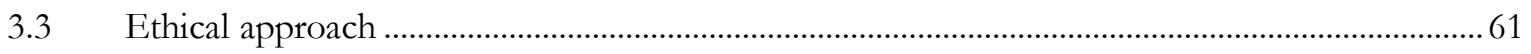

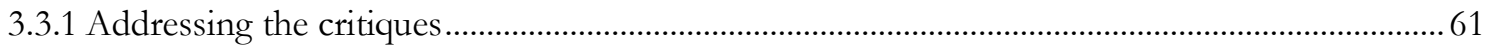

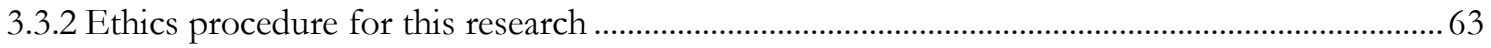

3.3.3 Strengths and weaknesses of ethical approach............................................................................... 64

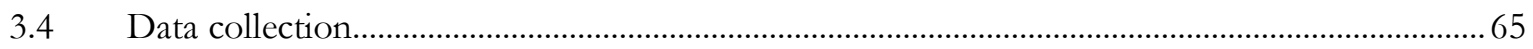

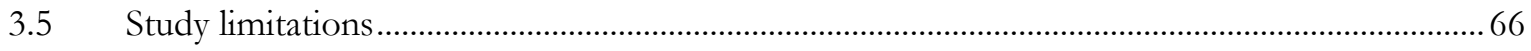

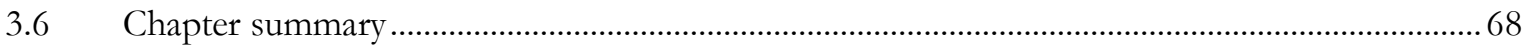

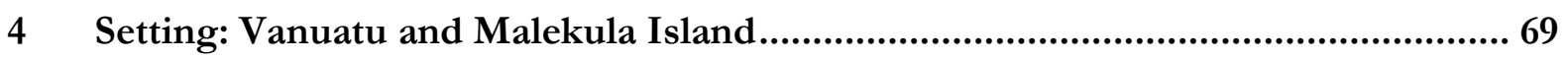

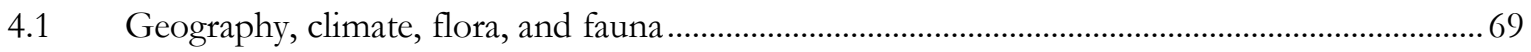

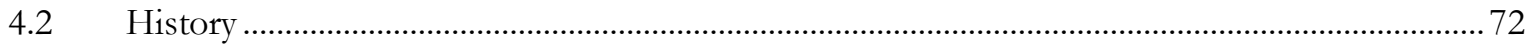

$4.3 \quad$ Current snapshot............................................................................................................. 74

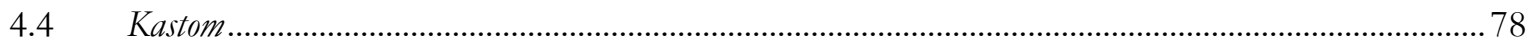

4.5 The Vanuatu Cultural Centre and the fieldworkers ………........................................................... 80

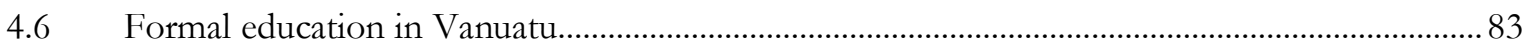

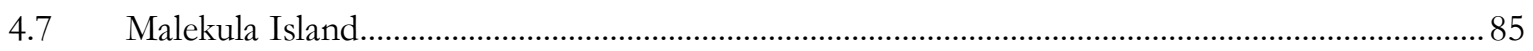

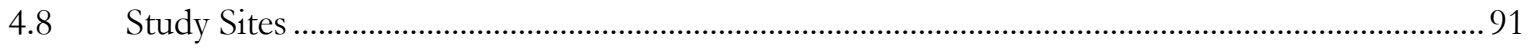

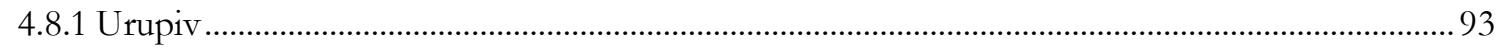

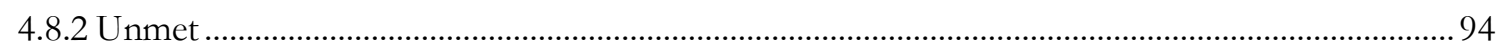

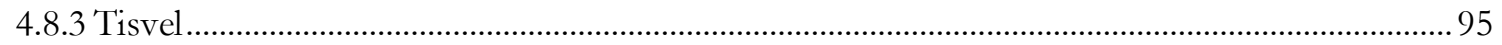

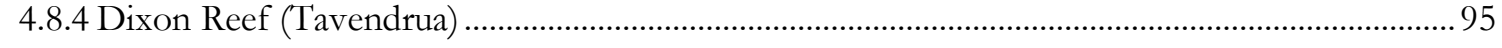

4.8.5 South West Bay (Lawa and Wintua) ............................................................................................. 97

4.9 Scoping study: Perceptions of TEK change on Malekula ...........................................................97

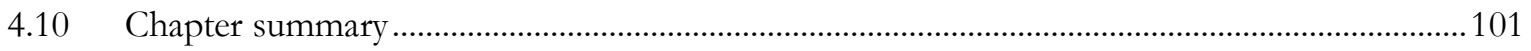

5 Explaining variation in TEK on Malekula..........................................................103

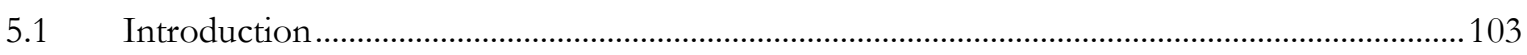

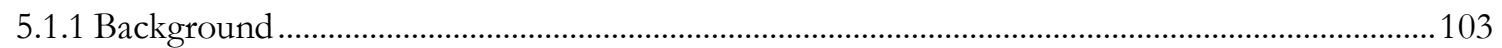




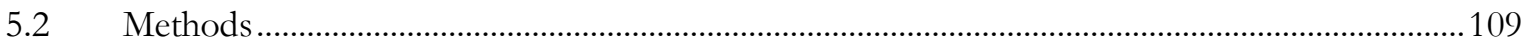

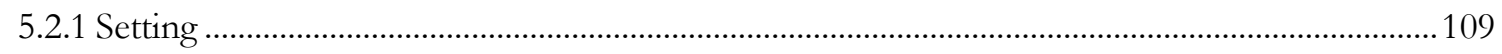

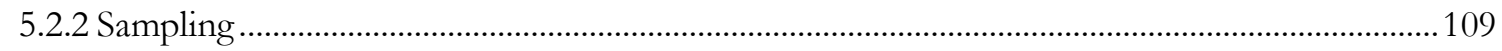

5.2.3 Variability in medicinal plant knowledge ............................................................................ 110

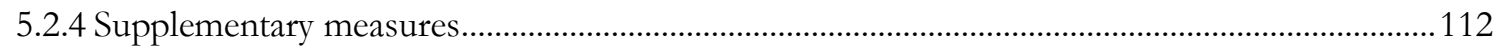

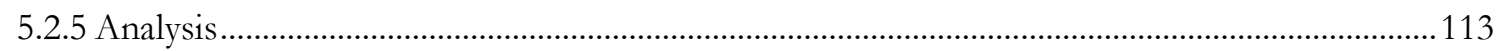

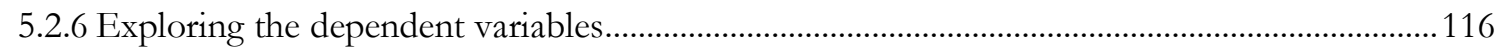

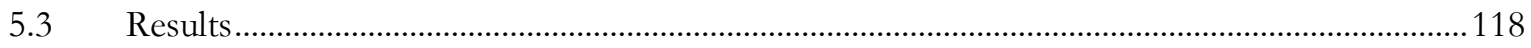

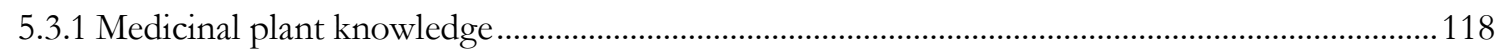

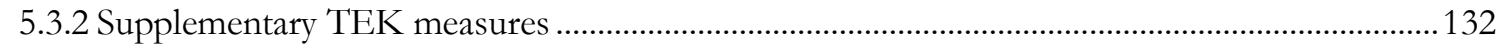

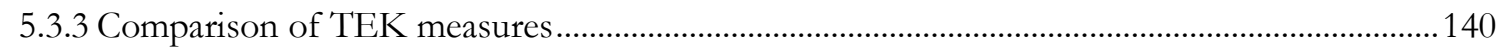

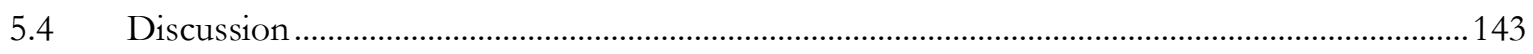

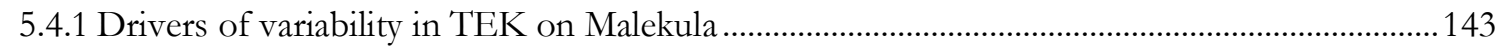

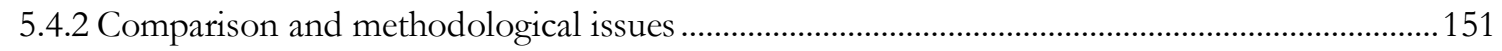

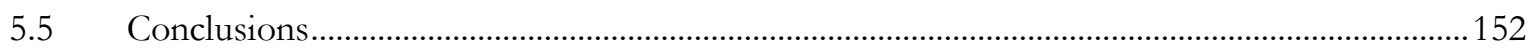

6 The cultural transmission of TEK on Malekula ...................................................155

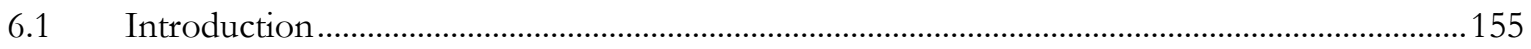

6.1.1 Cultural transmission: theoretical background ...................................................................156

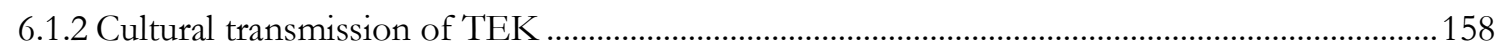

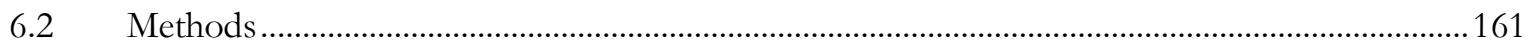

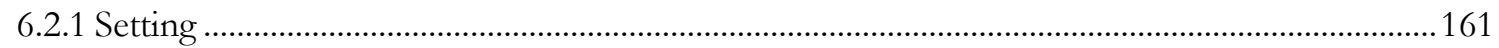

6.2.2 Mixed methods procedure and ethics..................................................................................... 161

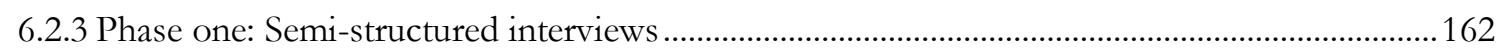

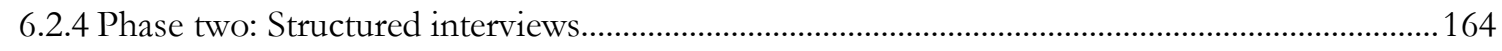

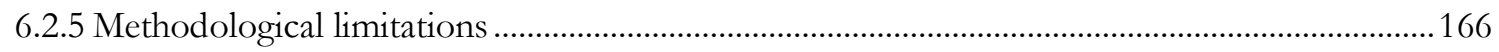

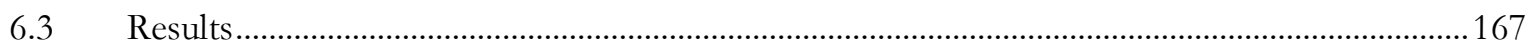

6.3.1 Cultural transmission in the focus communities (structured interviews)................................167 
6.3.2 Perceptions of changes in TEK transmission (semi-structured interviews).

6.4 Discussion.

6.4.1 Transmission pathways on Malekula

6.4.2 Changes in cultural transmission on Malekula

6.4.3 TEK transmission, TEK variation, and mixed methods approaches ..................................... 184

6.5 Conclusions

7 The value of TEK to formal school in Vanuatu

7.1 Background

7.2 Methods

7.2.1 Setting

7.2.2 Field methods

7.2.3 Methodological limitations

7.2.4 Analysis

7.3 Results

7.3.1 Could TEK be legitimately included into the formal school system? 195

7.3.2 How might TEK be integrated into the formal school system?

7.3.3 What are the possible barriers to teaching TEK in schools?.

7.4 Discussion

7.4.1 Barriers to including TEK in the formal education system ..................................................205

7.4.2 Moving forward with TEK maintenance and revitalisation ....................................................206

7.5 Conclusions

8 The in situ maintenance of TEK on Malekula

8.1 Introduction

8.1.1 International in situ TEK maintenance

8.1.2 In situ TEK maintenance in Vanuatu: The kastom schools

8.2 Methods

8.2.1 Setting 


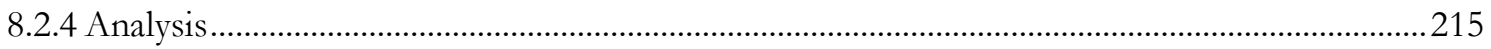

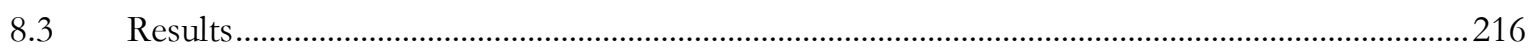

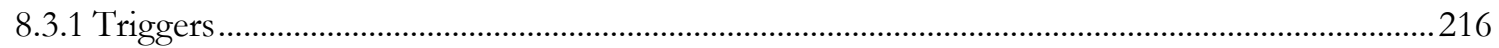

8.3.2 Challenges and barriers …………………………...............................................................2.

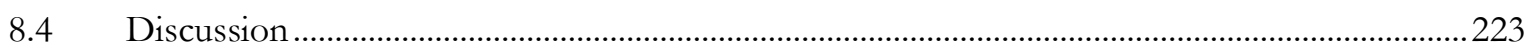

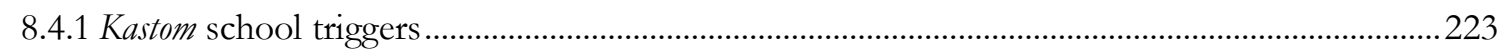

8.4.2 The conceptual barriers to the kastom schools .............................................................................224

8.4.3 The kastom schools and in situ TEK maintenance ..........................................................................227

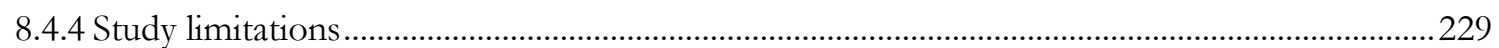

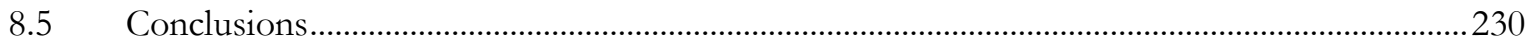

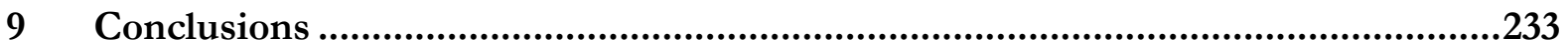

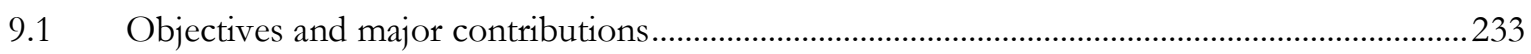

9.1.1 Objective one: To assess variation of TEK in four focus communities....................................23

9.1.2 Objective two: Cultural transmission of TEK on Malekula …………………..........................226

9.1.3 Objective 3(a): To assess perceptions of the value of TEK to formal school curricula ...........238

9.1.4 Objective 3(b): To assess community perceptions of in situ TEK conservation.........................240

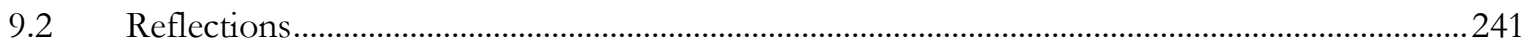

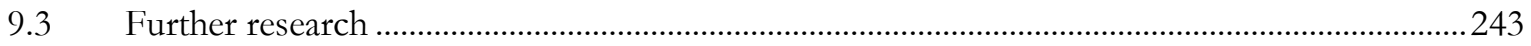

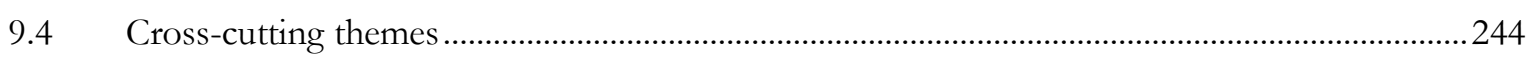

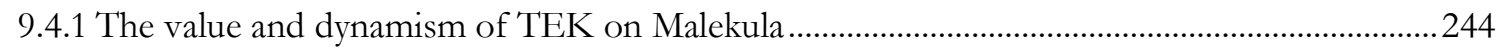

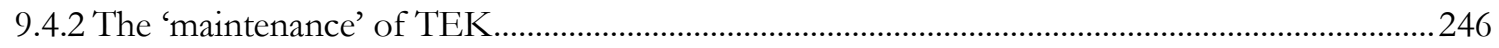

9.4.3 Multiple processes, multiple approaches......................................................................................24

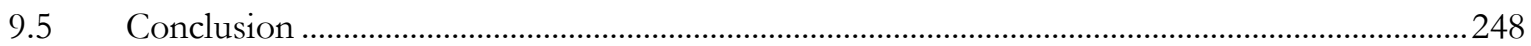

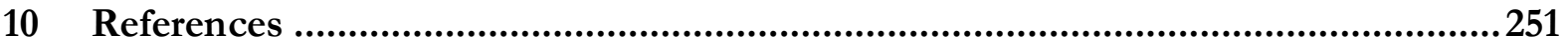

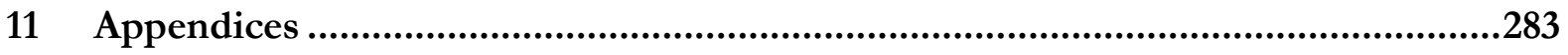

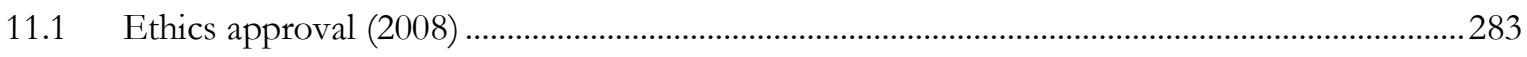

11.2 Information and informed consent forms (2008) …………………………………………....2. 284 
11.2.1 Community interviewees (information sheet and informed consent) ...............................225

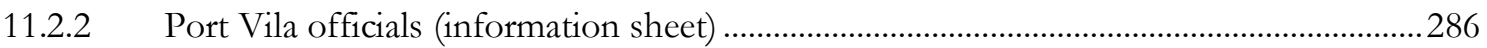

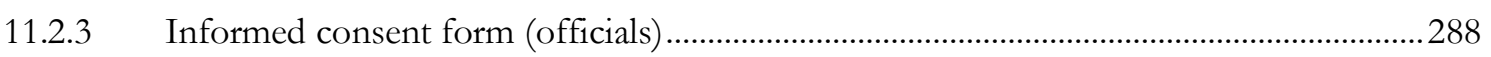

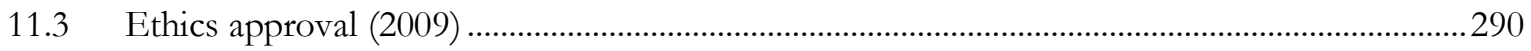

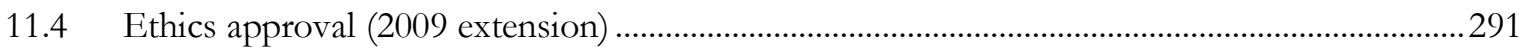

11.5 Information and informed consent forms (2009-2010) ……………………………………......2. 292

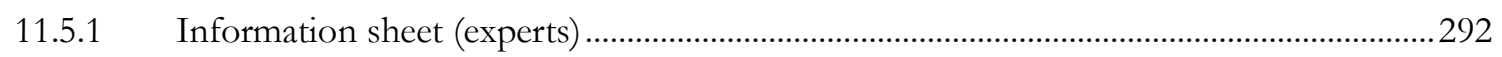

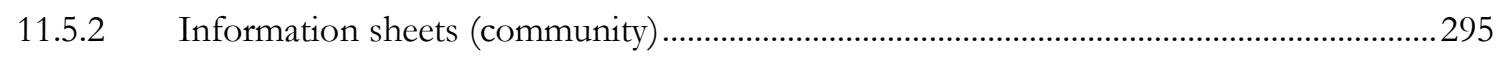

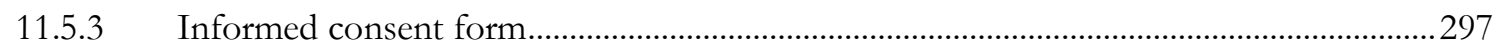

11.6 Semi-structured interview schedule (Chapter Seven) ………………………………………......29

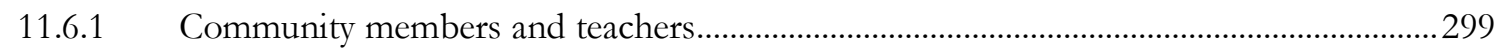

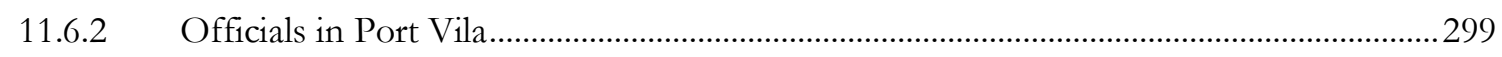

11.7 Semi-structured interview schedule 2009-2010.............................................................................. 301

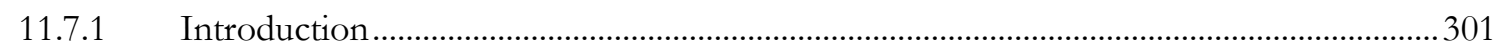

11.7.2 Freelisting (interview creation for Chapters Five and Six) .................................................. 301

11.7.3 TEK transmission (qualitative data for Chapter six).............................................................. 301

11.8 Semi-structured interview schedule (Chapter Eight)....................................................................... 303

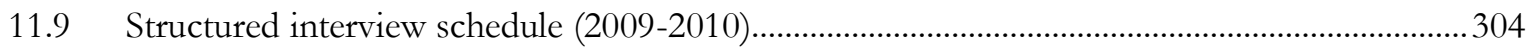

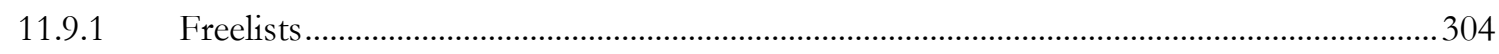

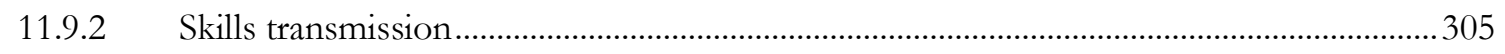

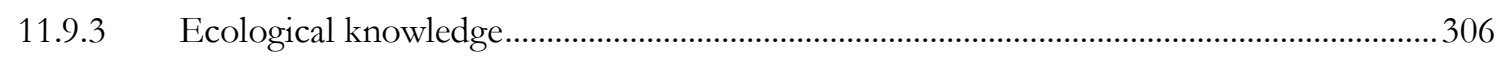

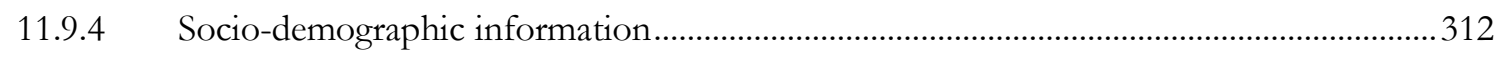

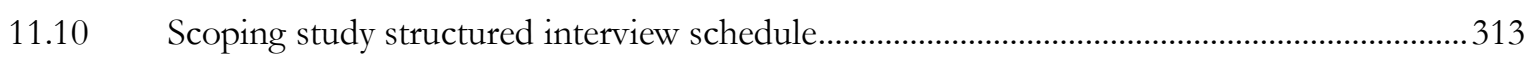

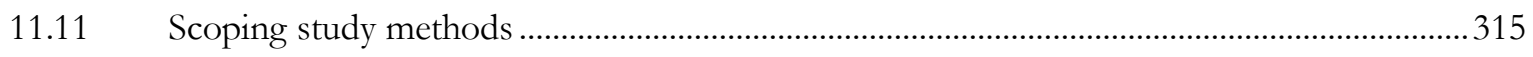

11.11.1 Sample

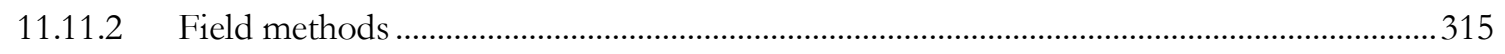

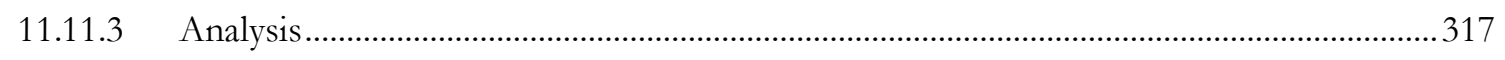

11.12 Vascular plant families cited during freelisting.......................................................................... 318

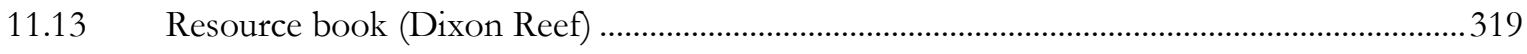




\section{Table of Tables}

Table 3.1: Summary of research methods used in this thesis .66

Table 4.1: Key characteristics of focus communities on Malekula Island 92

Table 4.2: Perceived drivers of TEK change on Malekula

Table 5.1: Predictor variables for measuring variation in TEK within and between communities... 107

Table 5.2: Participants in structured interview on TEK variation. 110

Table 5.3: 30 most salient illnesses derived from initial freelisting with 18 expert informants. 111

Table 5.4: Summary table of dependent variables 114

Table 5.5: Eleven basic models for testing via AIC analysis.

Table 5.6: Descriptive statistics for medicinal plants cited in ten illness domains. 120

Table 5.7: Bivariate correlations of dependent variables with predictor variables. 121

Table 5.8: Model ranking for LogRichness of citations from ten illness freelists 124

Table 5.9: Multiple regression model for LogRichness under Model Eight. 124

Table 5.10: AIC model ranking for Individual-level Shannon diversity index score 125

Table 5.11: Multiple regression model for individual-level Shannon score under Model Eight. 126

Table 5.12: Comparison between categories based on Model Eight 127

Table 5.13: AIC model ranking for square root reef fish citation abundance 134

Table 5.14: Multiple regression model for square root reef fish citation abundance under Model One

Table 5.15: AIC model ranking for score in ecological knowledge test. 137

Table 5.16: Multiple regression model for percentage score in ecological knowledge quiz under Hypothesis One..

Table 5.17: AIC model ranking for proficiency scores 139 
Table 5.18: Multiple regression for proficiency scores under Model One

Table 5.19: Bivariate Pearson's correlations between dependent variables

Table 5.20: Summary of multiple regression results of best models for four dependent variables... 142

Table 6.1: A model of cultural transmission (after Hewlett and Cavalli-Sforza 1986) 157

Table 6.2: Participants for structured interview on TEK transmission 164

Table 6.3: 18 key skills included in structured interview 165

Table 6.4: Percentage of responses per skill per mode of transmission 170

Table 6.5: Pearson's correlation of TEK measures with individual percentage of each transmission pathway cited

Table 6.6: Median age of initial skill transmission of 18 key skills 172

Table 6.7: Major location for teaching TEK skills, per domain (percentage of total responses) 174

Table 6.8: Summary of perceived intergenerational changes in transmission of TEK knowledge and skills, categorised into the developmental niche components 176

Table 7.1: Interviewee characteristics for semi-structured interviews. 193

Table 7.2: TEK subject domains for inclusion in the school curriculum 198

Table 7.3: Barriers to the inclusion of TEK in school on Malekula 200

Table 8.1: Key characteristics of three kastom schools on Malekula Island

Table 11.1: Participants in structured interview (Phase one)..... 315

Table 11.2: Domains of knowledge for inclusion in structured interview. 


\section{Table of Figures}

Figure 2.1 : Levels of analysis in traditional ecological knowledge and management systems (after

Berkes 2008)

Figure 4.1: South Pacific and Vanuatu

Figure 4.2: Malekula Island, with focus communities

Figure 4.3 Actively spoken languages on Malekula

Figure 4.4: Avava-speaking locations past and present.

Figure 4.6: Perceived loss of TEK on Malekula (historical and future), utilising trend analysis technique 98

Figure 4.7: Perceived changes in TEK amongst different subject domains......

Figure 5.1: Number of citations per life form

Figure 5.2: Richness of cited medicinal plants across ten freelists, per community and gender.

Figure 5.3: Mean percentage of cited plants cultivated, per age cohort.

Figure 5.4: Mao-Tao rarefaction curves of expected richness vs. number of informants with variables under hypothesis eight

Figure 5.5: Jack1 and MMMeans richness estimators against Mao-Tao species accumulation curve 132

Figure 5.6: Abundance of reef fish citations per community, split via gender.

Figure 5.7: Mean percentage score per section of ecological knowledge quiz

Figure 5.8: Scores in ecological knowledge quiz, per community and gender 136

Figure 6.1: Schematic diagram of data collection methods and integration...... 161

Figure 6.2: Percentage of respondents recording at least one transmission event for 18 ethnobotanical skills. 168 
Figure 6.3: Percentage of vertical transmission events for all 18 skills, split via gender of respondents.

Figure 6.4: Major place of learning key skills, by community and gender ........................................ 174

Figure 7.1: Percentage of respondents per grouping who support the teaching of TEK in formal

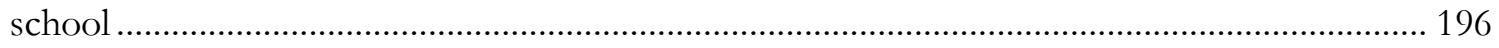

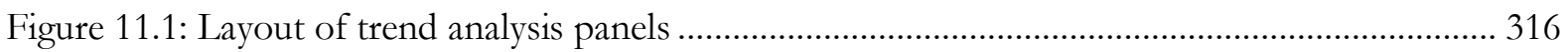




\section{Table of Plates}

Plate 4.1: James Teslo, a fieldworker from interior South West Bay, with pigs in his 'pig bank' ......... 82

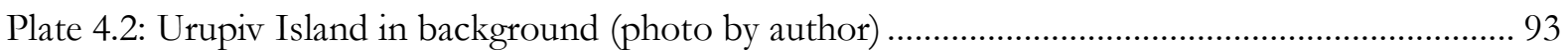

Plate 5.1: Interviewing informant using Likert scale (photo by author) ........................................... 113

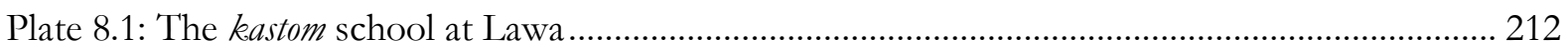




\section{Introduction}

\subsection{A broad location}

It is hard to imagine a time when the environmental and social challenges facing human society have been so urgent, so visible, or so obdurate. As we have pursued the gains in material wellbeing that characterise the modern era, we have rapidly depleted stores of accumulated natural and cultural capital (Pretty 2011). The resulting decline in biodiversity continues at unprecedented rates, and demand for natural capital continues to outstrip supply by $25 \%$ and rising (Pollard et al. 2010). The cultural diversity of the human species, too, is rapidly descending toward 'monocultures of the mind', with at least half of the world's 6,909 languages in danger of extinction and a language disappearing every ten days (Harrison 2007; Krauss 2007; Shiva 2006). Some authors (e.g. Steffen 2007) suggest that the extensive damage we have inflicted on the bio- and ethnospheres over the last two hundred years constitutes a distinct period of the earth's history, the 'anthropocene'. This is both an indictment on our rapid progress toward global homogenisation as well as an indication of the scale of the challenge we now face in attaining a sustainable future.

The current crises of cultural and biological diversity have prompted significant conceptual progress from academics and policy makers (Pretty 2011). At the heart of recent approaches to sustainability science is the recognition that human-environment systems are fundamentally linked (Perrings 2007). In some circumstances these 'social-ecological systems' (c.f. Westley et al. 2002) can be resilient to external shocks or perturbations, but erosion of natural and cultural capital can lead to increased vulnerability and reduced adaptive capacity (Adger 2003; Folke et al. 2002). Our emerging understanding of the dynamics of resilience in socio-ecological systems suggests that solutions lie not in the endorsement of universally applied panacea solutions (Ostrom et al. 2007), but rather the promotion of diversity and interdisciplinary enquiry (Kates et al. 2001). In particular, the role of local and indigenous systems of knowledge in supporting the resilience and adaptive capacity of socialecological systems has emerged as one of the most important (and controversial) discussions of the past three decades (Stephenson and Moller 2009; Turner and Berkes 2006b).

The increase in academic and policy interest in local and indigenous knowledge systems has been driven, in part, by the recognition of the links between cultural, linguistic and biological diversity. 
Luisa Maffi and colleagues have defined this 'biocultural' diversity as a new field of research and action, noting that it encompasses "...the diversity of life in all its manifestations... which are interrelated within a complex socio-ecological system" (Maffi and Woodley 2010: p 5). ${ }^{1}$ These links are evident at broad spatial scales: of the nine countries that harbour $60 \%$ of the world's languages, six are also centres of unique plant and animal species (Posey 2001). Evidence has also accumulated at a local scale: for example, numerous studies have noted that local and indigenous societies have played a key role in creating and maintaining biodiversity in many areas of the world (Zent and Zent 2007). Moreover, because indigenous peoples occupy many of the world's most important biodiversity hotspots, there is little doubt that indigenous ways of knowing and understanding the world will be a critical facet of natural resource management in the future (Maffi and Woodley 2010). In promoting the importance of indigenous and local knowledge, commentators do not typically argue for the abandonment of scientific, evidence-based, approaches to policy and practice. Rather, authors emphasise the importance of deploying the full range adaptive tools developed by various societies over millennia (e.g. Berkes and Jolly 2001), and for the ongoing maintenance of “...a diversity of ways of thinking about problems" (Ford and Martinez 2000: p 1250).

In short, we find ourselves at an interesting juncture. At precisely the time that we are beginning to understand the importance of indigenous knowledge systems, both natural and cultural capital are at risk amid pervasive global change. This thesis is located in the niche created by these intersecting concerns, and begins with the observation that, for much of the world, the erosion, maintenance, and revitalisation of indigenous knowledge systems are critical issues for the sustainable management of resources into the future.

\subsection{The significance of this research}

The subset of indigenous knowledge concerning the environment is termed traditional ecological knowledge (henceforth TEK, fully defined in Section 2.1). TEK is a valuable facet of biocultural diversity, and is based in contact between humans and their surrounding environment over millennia. As such, TEK represents an important store of knowledge and practice for the sustainable use and management of the environment (Berkes 2008), and has been widely utilised over recent decades toward aims spanning from biodiversity conservation (Charnley et al. 2007) to participatory development (Sillitoe 1998). However TEK is at risk of erosion amid social and ecological change (Quinlan and Quinlan 2007). This thesis is a response to the ongoing erosion of TEK, and seeks to

\footnotetext{
1 There remain considerable issues with the use of 'biocultural diversity', largely because there is “...no single way to measure a term with so many meanings" (Cailion and Desgorges 2007: p 2921). The mechanisms underlying the links between biological and cultural diversity, the robustness of correlations at fine spatial scales, and the policy implications of links between biological and cultural diversity all warrant further research attention (Harmon and Loh 2008). It is, however, a useful conceptualisation, and will be used in passing throughout this thesis.
} 
fill four critical literature gaps surrounding the variation, transmission, and maintenance of TEK in small-scale rural communities.

This research is important for several reasons. First, TEK may be impacted by processes of cultural homogenisation and is likely to be eroding in many areas of the globe (Quinlan and Quinlan 2007). In order to understand drivers of TEK erosion, scholars have attempted to quantify variation of individual-level knowledge in association with socio-demographic indicators of modernisation (e.g. Reyes-García et al. 2005). Some of this research corroborates the hypothesis that TEK can erode under the influence of globalisation (e.g. Benz et al. 2000), but other studies have produced mixed or conflicting results (e.g. Zarger and Stepp 2004). For example, integration into market economies can impact on some domains of knowledge, while others persist (Godoy et al. 1998). These results indicate the inherently complex nature of cultural change, and also highlight methodological inconsistencies and difficulties in the measurement of TEK (Zent and Maffi 2009). There is a clear need for quantitative research that adds to knowledge of the ways in which modernisation affect TEK and addresses methodological concerns (Zent and Maffi 2009). Increasing the global spread of research is also important, because previous research has rarely been situated in Oceania, despite it being a hotspot of biocultural diversity (Reyes-García et al. 2007a). I address these literature gaps in Chapter Five of this thesis by assessing the drivers of variation in TEK on Malekula Island, Vanuatu.

Second, TEK variation at an individual-level must be situated within its wider cultural context (Ohmagari and Berkes 1997). Like all facets of culture, TEK is perpetuated and sustained by cultural transmission (Reyes-García et al. 2009). Changes in systems of cultural transmission are a key mechanism through which knowledge persists or erodes, and may vary across domains of TEK and socio-demographic groupings (Hewlett and Cavalli-Sforza 1986).Therefore understanding the cultural transmission of TEK is an important facet of describing the process of knowledge erosion, and will be critical to addressing TEK homogenisation (Zent 1999). However, few ethnobiological studies have focussed on the cultural transmission of TEK. I address this literature gap in Chapter Six with mixed-methods research on the cultural transmission of TEK on Malekula.

Third, the suspected erosion of TEK has driven some commentators to call for increased research attention to its maintenance and revitalisation (Heckler 2002; Zent 1999). Several authors have proposed specific mechanisms, such as top-down approaches that incorporate in the formal school system (Reyes-García et al. 2010), or bottom-up approaches such as in situ conservation of TEK (Agrawal 1995). However, viability and salience of these mechanisms at a community-level, especially regarding the potential barriers to the success of TEK maintenance measures, have not been tested. My research begins to fill this literature gap by outlining community perceptions of both bottom-up and top-down approaches to TEK maintenance (Chapters Seven and Eight). 
This research is situated on Malekula Island in the Republic of Vanuatu, for several reasons. In particular, Vanuatu is one of the world's hotspots of biocultural diversity, encompassing 106 languages and being situated on the eastern edge of the East Melanesia biodiversity hotspot (Conservation International 2009; Lynch and Crowley 2001). The erosion and persistence of TEK are critical issues in Vanuatu, as TEK underpins resource management (Hickey 2006b), adaptive capacity to natural hazards (Campbell 2006), and is a valuable source of ethnomedical practice (Bradacs 2011). Practically, working in Vanuatu allowed my participation in a broader study on TEK variation and change on Malekula, and to utilise the institutional infrastructure of the Vanuatu Cultural Centre (VCC) and their network of volunteer fieldworkers (described in Section 0). 


\subsection{Aim and objectives}

The core aim of this thesis is to understand processes of the variation, change and maintenance of TEK on Malekula Island, Vanuatu. In doing so, I address the literature gaps noted in the paragraphs above. These contributions, both theoretical and methodological, will be important in guiding future research and responses to TEK erosion.

The major objectives of this thesis are:

1. To assess variation in TEK in four focus communities.

a. To assess TEK variation in relation to socio-demographic explanatory variables.

b. To assess evidence of TEK change or erosion.

c. To assess whether different indicators of TEK reveal divergent patterns.

2. To investigate the cultural transmission of TEK on Malekula; to examine changes in the transmission pathway of TEK in the focus communities.

3. To assess community perceptions of the value of top-down and bottom-up approaches to TEK maintenance.

a. To assess perceptions of top-down measures for the maintenance and revitalisation of TEK, by assessing the value of TEK to formal school curricula.

b. To assess community perceptions of in situ TEK conservation by examining the triggers and barriers to three local kastom schools.

\subsection{Thesis structure}

This thesis is built around four core chapters that are presented as stand-alone, publication-oriented studies. Each of the chapters has its own theoretical and methodological approach to answering a distinct set of questions, and each addresses a specific research objective:

Chapter Five addresses objective one, and examines the variation of TEK in four focus communities on Malekula using a structured interview with 177 informants.

Chapter Six addresses objective two, and investigates the cultural transmission of TEK in the same communities using both quantitative and qualitative methodologies. 
Chapter Seven was published as McCarter and Gavin (2011), and addresses objective 3(a). This chapter uses semi-structured interview data to discuss perceptions of the value of TEK to formal school curricula in Vanuatu.

Chapter Eight addresses objective 3(b), and uses qualitative methodology to discuss three kastom schools (small local organisations for the maintenance and revitalisation of TEK) on Malekula.

These core chapters are prefaced by three introductory sections. Following this opening chapter, Chapter Two discusses the literature that underpins the thesis in some detail, including defining and delineating the concept of TEK. Chapter Three briefly outlines the overall methodological approach, including a consideration of my positionality and its impact on the research process.

Chapter Four introduces the setting of the research and outlines the geography, ecology, and history of Vanuatu and Malekula Island. This chapter also briefly details the results of a scoping study outlining perceptions of TEK change on Malekula.

The thesis concludes in Chapter Nine. In that section, I summarise the contributions of each of the core chapters before reflecting on the success of the methodological approach in addressing my objectives. I close the thesis by discussing three key cross-cutting themes that are woven throughout the thesis. 


\section{TEK: Definition, application, and current trends}

In this chapter I review the literature surrounding traditional ecological knowledge (TEK). My aim is to provide a broad theoretical basis for the core chapters that follow, in order to allow nuanced discussion of the concept of TEK and fully situate each chapter in the relevant literature. As such, the following sections cover a range of topics, and outline the definition of TEK, including some commonly-cited conceptual issues (Section 2.1); values of TEK within academia and beyond (Section 2.2); criticisms of TEK research (Section 2.3); and the current state of knowledge regarding the erosion, transmission, and maintenance of TEK (Sections 2.4 - 2.6). The final section of this chapter (Section 2.7) summarises the key findings of the literature review and emphasises the four literature gaps that are filled by the research presented in this thesis. In addition to this chapter, each core chapter is itself prefaced by a short introduction that builds on the literature reviewed here.

\subsection{Definition}

TEK is the central concept of this thesis. It has emerged over the past three decades as a distinct and well-researched domain of knowledge, and is now understood to be a critical facet of biocultural diversity (Berkes 2008; Brosi et al. 2007; Hunn 1999; Pierotti and Wildcat 2000). In this thesis I utilise the operational definition developed by Fikret Berkes and colleagues, who suggest that TEK be conceptualised as:

“...a cumulative body of knowledge, belief and practice, evolving by adaptive processes and handed down through generations by cultural transmission, about the relationship of living beings (including humans) with one another and with their environment" (Berkes 2008: p7). 
As such, the term 'TEK' represents the facets of indigenous and local knowledge that concern the environment, and is the product of millennia of human-environment interaction (Berkes et al. 2000; Hunn 1999).

Several intersecting points emerge from this definition. First, TEK is defined as a complex of knowledge, practice, and belief. The concept is therefore inherently broad, and covers multiple dimensions of cultural knowledge. This has been represented in Berkes (2008) as a heuristic 'nested egg' model, encompassing the multiple overlapping dimensions of TEK (Figure 2.1). The first layer consists of the empirical and place-based knowledge (for example, species identification) that is often held by local and indigenous people (demonstrated in Coe and Anderson 1996; Lewis 2003; Salick et al. 1997). These are often the aspects of TEK that are described in ethnobiological research, and are readily acceptable in a cross-cultural context. The second layer consists of local resource management practice, which utilises stores of local knowledge in combination with an appropriate sets of practices, tools, and techniques (e.g. Bridges and McClatchey 2009; Hickey 2003b; Johannes 1998). The third layer comprises the social institutions within which management practices and knowledge are encapsulated (e.g. Becker and Ostrom 1995; Dietz et al. 2003). These include institutions for the maintenance of norms, relationships, and values, which form the basis for collective action (Adger 2003). Finally, the outer layer represents the worldview and belief structures which 'shape environmental perceptions and gives meaning to observations of the environment' (Berkes 2008; Lertzman 2002). This layer is inextricable from culture more broadly.

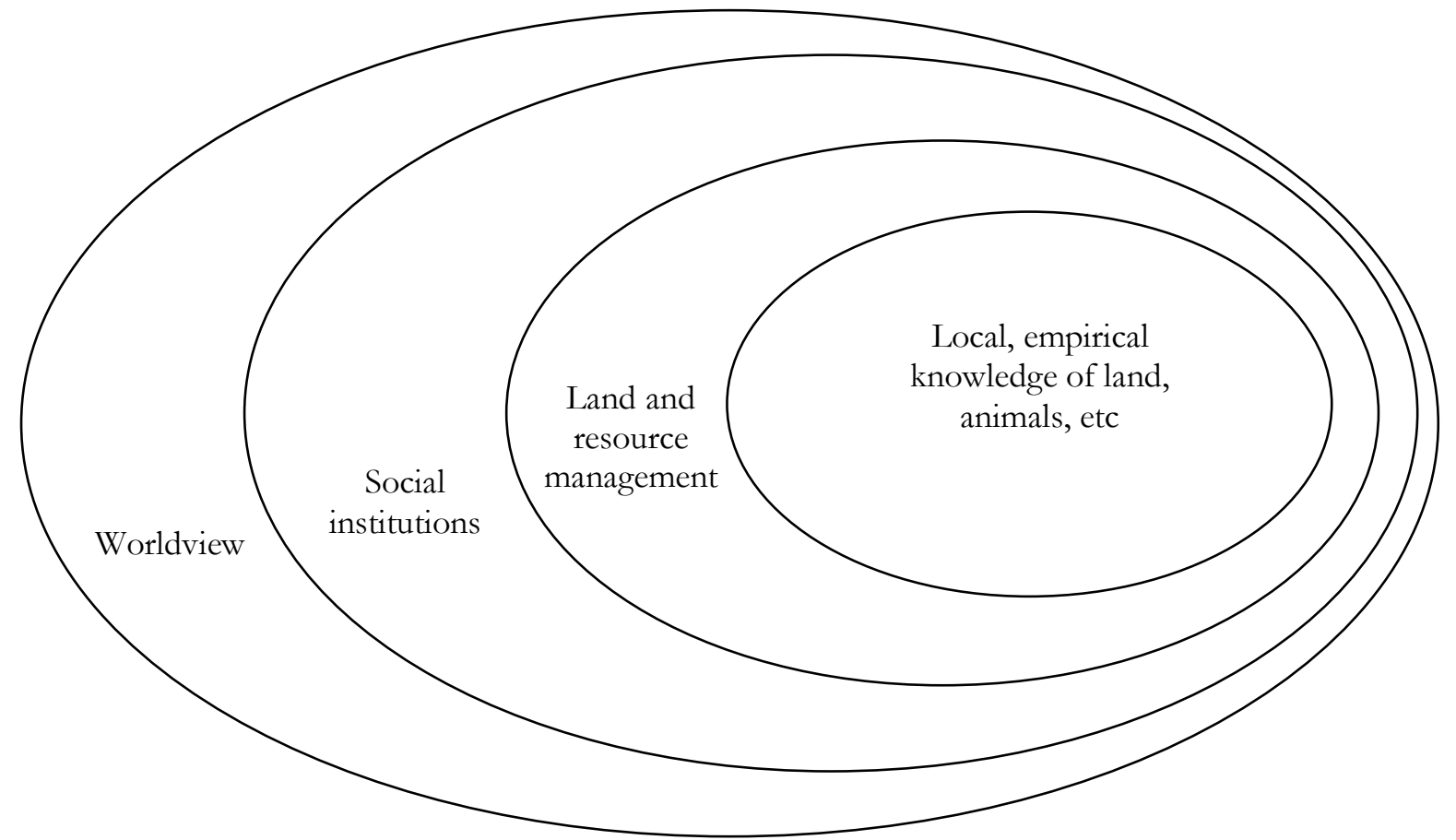

Figure 2.1: Levels of analysis in traditional ecological knowledge and management systems (after Berkes 2008) 
The second point stemming from Berkes' definition is that TEK is conceptualised as an adaptive body of knowledge. I do not mean to suggest here that TEK is universally well adapted to all social and ecological contexts (on the contrary, TEK is inherently local, a point I'll return to below). Rather, in developing over long periods of time in close relationship to the land and natural resources, TEK has by necessity developed through adaptation to local conditions. Berkes and Turner (2006) suggest that TEK, and specifically conservation and management knowledge, can develop through two nonmutually exclusive scenarios they term the 'depletion crisis model' and the 'ecological understanding model'. The former occurs following resource crises, which enforce the development of conservation knowledge through necessity: by crossing ecological limits (such as sustainable yields from fish stocks), TEK is learned and deployed to avoid repetitions in the future. This is likely to have been the case in areas such as remote Oceania, where fragile island ecosystems would have been rapidly depleted on initial colonisation (Diamond 2005; Jackson et al. 2001), resulting in continued habitation being unsustainable were it not for the development of conservation knowledge (Johannes 2002). The latter occurs over long periods of time, and suggests that human societies can develop sophisticated ecological understandings based on observation, incremental modification, and trial and error and monitoring, all of which are perpetuated through oral histories and discourse (Turner and Berkes 2006a).

As the previous paragraph suggests, TEK is also fundamentally dynamic. In this sense, the use of the term 'traditional' is something of a misnomer. TEK, like culture more broadly, is cumulative in nature and is susceptible to the influence of new ideas, ideologies, and information (Ellen et al. 2000). As such, 'tradition' does not refer to the inflexible transmission of knowledge from the past into the present; rather, it refers to the lens through which new information is interpreted and included into the contemporary context (Berkes 2008). For example, Ohmagari and Berkes (1997) observed at James Bay in Canada that: “...the Cree [local indigenous people] are eclectic about what they consider 'traditional'. For example, goose hunting is a traditional pursuit. The use of a modern shotgun, which is a tool only, does not change the fact, in the Cree mind, that the goose hunt is defined as a traditional activity" ( $p$ 203). The dynamism of TEK can be obscured by efforts at 'conservation' of knowledge and practice, or by dialogues surrounding the 'erosion' of knowledge (Bates 2009).

Third, TEK is handed down through generations through cultural transmission. There is a large anthropological literature on cultural transmission, which has been defined as the "...process of social reproduction in which the culture's technological knowledge, behaviour patterns, cosmological beliefs, etc., are communicated and acquired" (Cavalli-Sforza et al. 1982: p 19). TEK transmission has, in the past, been predominantly oral and experiential in nature (Ruddle 1993); however, this may be changing due to the influence of Western norms and values, such as those introduced through 
formal school systems (Ohmagari and Berkes 1997). Systems of transmission are highly structured and based around a hierarchical progress from novice to expert (Ruddle and Chesterfield 1977). Quantitative studies have suggested that most TEK transmission occurs between parents and children (vertical transmission; e.g. Eyssartier et al. 2008; Hewlett and Cavalli-Sforza 1986), although ethnographic accounts often stress the role of horizontal transmission from peers (Aunger 2000; Lancy 1996; Rogoff 1981). Importantly, as suggested in the definition of TEK above, questions of TEK erosion cannot be considered in isolation from processes of cultural transmission and acquisition of knowledge (Zarger and Stepp 2004). I examine cultural transmission of TEK on Malekula Island in some depth in Chapter Six.

Fourth, in concentrating on the relationships between living beings and the environment, TEK is holistic in nature (Reichel-Dolmatoff 1976; Salmon 2000). TEK is tied to specific localities, meaning that all aspects of the physical space can be considered as part of the community, including plants, animals, and landforms (Pierotti and Wildcat 2000). This puts it in contrast to contemporary western attitudes, in which humans are often conceived to be autonomous from the remainder of the natural world (Pierotti and Wildcat 2000). It has been argued that this tendency means that TEK systems are more likely to display conservation-oriented behaviour than those of western societies (Salmon 2000), though this is disputed (cf. Redford and Stearman 1993). I return to this argument below, however I note that the holistic outlook of TEK is somewhat more congruent with some emerging literatures from the western science paradigm such as those on linked social-ecological systems (Berkes and Folke 1998; Carpenter et al. 2001; Folke et al. 2007; Gadgil et al. 1998) and biocultural diversity (Cocks 2006; Maffi 2005; Turner et al. 2003). The holistic nature of TEK can make it problematic to represent through academy-based literature, as separating the technical from the non-technical or the rational from the non-rational is difficult (Ellen and Harris 2000).

Fifth, in defining TEK as having accumulated within cultures based on contact with their environment, Berkes' definition above hints at the inherently local nature of TEK. TEK is developed through contact with a particular local environment, and therefore the empirical information within TEK systems is unlikely to be widely applicable outside that context (Zent and Maffi 2009). This characteristic of TEK opens a critical node of discussion, which is the degree to which broad lessons can be drawn from the various documented examples of TEK and applied to other areas of the globe (Hunn 1999). As noted by Ellen and Harris (2000): “...the salience of the same series of...plant species for a particular indigenous people compared with global science is likely to be very different, as their taxonomic and utilitarian linkages are on the one hand, local, culture-specific and restricted, and on the other, global, culturally de-contextualised, and extensive" (p 4). It is argued here, and elsewhere, that the inherently local nature of TEK, rather than representing a weakness, 
reminds us of the importance of using place-based knowledge to guide actions toward the natural world (Pretty 2011).

\subsubsection{A note on alternative phrases}

Throughout this thesis I use the phrase 'TEK' to discuss local and indigenous sources of information on environmental processes and elements. There has been, however, considerable debate about the phrasing of the concept, and various commentators have disagreed on a fundamental level with each of the 'traditional', 'ecological', and 'knowledge' components (Nadasdy 1999). In particular, the use of 'traditional' has been criticised as denoting $19^{\text {th }}$ century attitudes of 'simple, savage and static' (Warren 1995: p 13), and thus inadequately portraying the dynamic, contemporary, and relevant nature of TEK (Morrow and Hensel 1992). 'Ecological' too has been disputed as narrowly representing the branch of western science concerned with interrelationships within the biophysical realm, which may exclude humans (Berkes 2008). As noted above, this may be opposed to indigenous views of the world and the concept of TEK itself (Nadasdy 1999). Finally, 'knowledge' too has been argued to poorly represent the multi-dimensional nature of the concept as represented in Figure 2.1, in which TEK is as much a 'way of life' as a discrete and empirical product of human intellect (Nadasdy 1999).

In response, authors have used a range of broadly synonymous terms (Wenzel 1999). These have developed over recent years as debates about the nature of TEK and the terminology that surround it have become more sophisticated (Berkes 2008). Several prefer the terms 'indigenous knowledge' or 'indigenous ecological knowledge', arguing that they better reflect the situated and dynamic nature of the knowledge (e.g. Ellen et al. 2000; Sillitoe 2002; Warren 1995). The definition of the term indigenous, however, is highly contentious, and deserves an entire literature review in its own right (Sundar 2002). In particular, the usage of terms such as indigenous knowledge have been criticised, on the basis that they imply a kind of knowledge that is restricted to indigenous peoples only, and presuppose there is a discrete kind of knowledge which can be labelled as 'indigenous' (Agrawal 1995; Berkes 2008). As observed by Sundar (2002) while discussing 'indigenous' in the Indian context: “...to use the term indigenous...is to be complicit in a history of someone else's making, to accept a category of Hindu and tribal defined by a century and more of colonial knowledge creation, to deny the possibilities of common construction and common destruction by indigenous and nonindigenous alike" ( $p$ 81). Others prefer the terms ecoliteracy (Pilgrim et al. 2007), local ecological knowledge (Davis and Wagner 2003), or ethnoecological knowledge (Toledo 2002), however these terms have yet to find widespread acceptance in the literature. Berkes (2008: p 5) notes that the aboriginal people of the Canadian north prefer the use of 'land' to 'ecological': for them, the land is more than a physical landscape, and includes the living environment as well. In this case, the author 
responds by broadening his definition of 'ecological' to encompass this sense, while noting that ecological has a greater meaning to English speakers than land.

I acknowledge that there are potentially significant issues with the conceptualisation and definition of TEK. This debate is an important, as the terminology that is used in this context reveals something of the authors approach to the subject (Ellen and Harris 2000). This theme recurs throughout the thesis, and I discuss the implications of these challenges in the conclusion (Section 9.2). My position is, however, that for all of the definitional issues inherent in the use of the term, TEK is wellestablished in the literature and has a correspondingly solid theoretical framework. Moreover, there are no more universally-accepted alternatives that are more appropriate to this discussion. Therefore I use the 'TEK' throughout this thesis, and find that it provides a useful point of departure for discussing the knowledge, practice, and belief of community members in Vanuatu related to the environment. Where necessary, I also use the terms 'indigenous knowledge' or 'traditional knowledge'. Like Wenzel (1999), I interpret these terms to include TEK, and note they are understood to be a significant, if not exclusive, part of culture more broadly (Berkes 2008; Warren 1995; Wenzel 1999).

\subsection{Why TEK? Values of TEK in contemporary academia}

TEK is an important resource, and has value in a wide variety of contexts. Below, I detail six fields where TEK has applied value, in order to demonstrate the ways in which TEK has been utilised and deployed in the literature (and why it is such an important resource).

Ecological information: Scientists conducting botanical and zoological surveys or inventories in the field have often leveraged the knowledge of local naturalists to locate, collect and identify new species (e.g. Diamond and Bishop 1999). Empirical information contained in systems of TEK may include detailed knowledge on natural histories, behaviours, and species interrelationships, for which data may not exist in Western science (Berkes 2008). As noted, a good example is the work of Johannes (1981), whose detailed research showed that local fishers in Palau maintained TEK that surpassed (by a significant margin) that of the academic world at the time. Another pertinent example is given by the ongoing work of Henrik Moller and colleagues in a cross-cultural partnership between university researchers and local Māori in New Zealand (Moller 2009; Moller et al. 2009a; Moller et al. 2010; Moller et al. 2009b). This work has utilised a combination of scientific methodologies and matauranga Mäori (traditional knowledge) to examine the harvest of tïti (Puffinus griseus), and has produced novel insights, including description of the impact of El Nino weather systems on seabird nesting density (Pretty 2011; Stephenson and Moller 2009). 
Medicine: The accumulated folk knowledge and use of plants, animals and fungi for medicine is of central importance to much of the world's population. Recent estimates suggest that, in some areas of the world, $80 \%$ of the population rely on traditional ethnomedical knowledge (World Health Organisation 2008). As such, this knowledge is central to the wellbeing of a significant percentage of the world's population, especially in areas where access to western biomedical care is limited (Voeks and Leony 2004). The traditional ethnomedical knowledge of healers has also proved to be of significant value for pharmacological development, and has resulted in the development of drugs for cancer and diabetes, amongst others (Cox 2000). Apart from obvious human benefits, the commercial importance of such knowledge could be extremely significant: Voeks and Leony (2004) suggest the market could be worth $\$ 3.2$ to 4.7 billion US dollars. The considerable market value of ethnomedical knowledge has spurred a significant resurgence in interest in medicinal TEK in recent years, particularly in the Amazon Basin (Voeks 2004). This has been accompanied by concern surrounding the protection of the intellectual property of the TEK holders against biopiracy (Posey and Dutfield 1996) and the unsustainable commercial extraction of useful medicinal plants (Cox 1999).

Natural resource management: There are many documented examples of traditional management regimes that promote practices which are consistent with goals of conservation and sustainability. Specific practices include actively managing consumption pressure on key species (Smith et al. 2007); preserving a diversity of famine-ready crops (Campbell 1990); use of small-scale patchiness and fallow management (Niamir-Fuller 1998); and the use of transitory rotation systems of use in fragile ecosystems (Campbell 2006); wetland burning for enhancing biodiversity and ecosystem resilience (McGregor et al. 2010; Prober et al. 2011); and communal property arrangements (Alcorn and Toledo 1998; Ostrom 1990). TEK may also effectively contribute where the extensive datasets needed for forming management plans are not available, as in the management of topical nearshore fisheries (Johannes 1998), or the conservation management of arid areas (Kellner and Bosch 2003).

Disaster risk reduction: A growing body of literature emphasises the importance of TEK in disaster risk reduction (Mercer et al. 2010). This has been especially emphasised in small island developing states, which are typically characterised by inherent vulnerability and a high propensity to environmental hazards (Pelling and Uitto 2001). TEK has been documented as an important component of volcano hazard management (Cronin et al. 2004); as supporting a high diversity of crops which offer resilience to extreme weather events (Campbell 1990); as providing the essential knowledge for the construction of resilient housing, which may withstand and be regenerated more efficiently after extreme weather events (Campbell 2006; Schilderman 2004); and through supporting the social networks which provide coping strategies in the event of large natural disasters, such as the eruption of Mt Pinatubo in 1991 (Seitz 1998). TEK as a source of resilience to extreme weather 
events is likely to become more important over the coming years, as the challenges posed by global climate change are realised (Adger 2003; Alexander et al. 2011).

Development: The inclusion of indigenous knowledge and TEK into development policy and practice was heralded as a 'revolution' and was associated with a shift toward participatory, bottomup approaches (Escobar 1995; Sillitoe 1998). The appropriate use of TEK was noted, amongst other benefits, to promote local involvement and empowerment (Brokensha et al. 1980; Warren et al. 1995), allow diversification of income streams through knowledge of non-timber forest products and medicines (Begossi et al. 2000; Berkes 2008), and promote and maintain resilient and diverse food supplies (Nazarea 1998). However, the incorporation of indigenous knowledge into development strategies has met with limited success (Briggs 2005), which has been variously ascribed to issues of trust in different knowledge forms (Briggs and Sharp 2004; Sillitoe 2010), inadequate recognition of community heterogeneity (Agrawal and Gibson 1999), or the use of TEK to promote fundamentally top-down, etic, aims (Heckler 2007).

Formal education: Education systems in many areas of the world fail to adequately account for cultural diversity, and often focus on teaching a limited set of curricula derived from western, colonial, models (Luke 2009; Teasdale et al. 2005; Thaman 2002). As a result, student performance is affected, and formal schooling can remain an ineffective and traumatic experience for indigenous peoples (Kimmerer 2002; Snively and Corsiglia 2001). There is a burgeoning literature examining the positive impact of contextualising education systems, so as to better make them more directly relevant and provide a better sense of place and identity to students (Castagno and Brayboy 2008; Ruiz-Mallen et al. 2010; Ruiz-Mallen et al. 2009). Systems that integrate TEK and indigenous knowledge can enhance understanding of curricular knowledge, in part through linking the theoretical knowledge of the classroom with practical, lived, reality (Castagno and Brayboy 2008; Reyes-García et al. 2010). Moreover, contextualised education systems can increase knowledge acquisition by empowering students, reinforcing learner self-esteem, and maintaining individual and collective cultural identity (Lipka 1994; Reyes-García et al. 2010; UNICEF 2003). The inclusion of TEK in formal schooling may also increase broader environmental awareness in pupils, potentially leading to an increased capacity for knowledge and pro-environmental action (Ruiz-Mallen et al. 2009; Taylor and Mulhall 2001).

TEK also has value to other fields, such as biodiversity conservation (Byers et al. 2001; Charnley et al. 2007), ecological engineering (Martin et al. 2010), and environmental ethics (Lertzman 2002; Suzuki and McConnell 1997). Moreover, because TEK is also a manifestation of human-environment links over millennia, TEK is a critical indicator of the health of biocultural diversity more broadly (Zent and Maffi 2009). 
Scholars have also argued for the importance of TEK in maintaining the resilience of some socialecological systems. Resilience refers to the ability of a given system to undergo disturbance and maintain its function and controls (Carpenter et al. 2001). Resilience is built through the interaction of several ecological and cultural components, including natural capital, which delivers a flow of ecosystem goods and services; social capital, in the form of relationships, trust, and institutions for collective action; buman capital, in the form of knowledge and skills for human wellbeing; and physical and financial capital that provides structure and economic resources (Pretty 2011; Pretty and Smith 2004). In many areas of the world, TEK is a critical component of both social capital (because it is intimately related to social networks and institutions: e.g. Pretty and Smith 2004) and human capital (because it often comprises a large body of skills and knowledge: e.g. Reyes-García et al. 2006b). Consequently, TEK may serve to increase the adaptive capacity (and thus the resilience) of socioecological systems, by providing coping mechanisms and a large array of traditional practices (Berkes and Folke 1998; Berkes and Folke 2002; Tompkins and Adger 2004). Specific aspects of TEK that support resilience include its dynamic and iterative nature, its awareness of ecological feedback loops, its historical depth, and its underlying worldviews (which are often holistic and support robust codes of environmental ethics) (Folke et al. 1998).

In describing the value of TEK in the utilitarian terms above, I do not wish to obscure the fundamental value of TEK as a facet of cultural diversity. The diversity of peoples, languages, and knowledge has an incalculable and intrinsic importance in its own right, and does not need the recognition and validation of academia to have value. Harmon (2002: p 45) suggests a number of reasons why cultural diversity should be maintained, including that it acts as a reservoir for human creativity; because it is a matter for social justice and a recognition that people have a right to cultural self-determination; and because cultural diversity is critical in the formation of identity, and therefore of well-being. Moreover, cultural diversity delivers large economic benefits to societies worldwide, both as a source of income (i.e. through cultural tourism) and as an avoidance of costs (by offering novel solutions to social problems that would otherwise require outlays of money). As such, cultural diversity is now considered to be a key pillar of sustainable development in many areas of the world (United Nations 2011). For example, in the Pacific, cultural diversity as an expression of creativity is being harnessed for economic growth and self-determination throughout the region (SPC 2010). 


\subsection{Controversies and representation in TEK research}

The value of TEK is, therefore, well established in the literature. However, scholars have noted a number of interlinked conceptual and methodological tensions inherent in research into TEK, which I briefly outline here. These tensions broadly coalesce into three themes involving the placement of TEK against western or scientific knowledge; the description and representation of TEK in the academic literature; and concerns about the nature of the research process from TEK holders and indigenous peoples.

\subsubsection{TEK and 'western' knowledge}

There are a number of ongoing debates around the validity and efficacy of TEK and indigenous knowledge, within which TEK is often placed in opposition to 'western', scientific knowledge (Nature 1999). Prominent examples have occurred within the field of biodiversity conservation, where there has been considerable disagreement about the 'conservation ethic' of indigenous societies (Johannes 2002), as well as broader discussions around the role in which TEK and local peoples should play in the creation and management of protected areas (Brechin et al. 2002; Drew 2005; Mascia et al. 2003; Nabhan 1995). Some, such as Schwartzman et al. (2000) argue for the extensive role of indigenous peoples in conservation initiatives, while others, such as Terborgh (2000) and Redford and Sanderson (2000) note that such approaches may serve to obscure the wider sociopolitical processes which drive ecosystem degradation. Similarly, in the arena of fisheries management, Foale (2006a) has observed that while the inhabitants of Lihir Island (Papua New Guinea) hold in-depth ecological knowledge of the reef, their TEK is lacking in understanding of certain fundamental characteristics that would be necessary for sustainable management of resources.

These debates are complex and context-dependent. Arguments for the use of TEK have been framed in the past by popular representations of indigenous people as 'noble savages', and have been predicated on the perception that indigenous people are close to nature and 'live in balance' with the environment (Berkes 2008). In reality, not all groups who have extensive TEK of the local environment display conservation-minded behaviour (Berkes 2008; Redford and Stearman 1993), and indigenous societies have commonly had significant impacts on biodiversity (Flannery 2002). In New Zealand, it has been argued that the renaissance of matauranga Mãori (Māori traditional knowledge) has led to a politicisation of the science process (Don 2010; Marie and Haig 2009). This, it is contended, has impacted on the quality of natural resource governance and "...elevate[d] a traditional knowledge system that bears little resemblance to evidence-based science" (Don 2010: p 43). 
On the other hand, Schwartzman et al. (2000) argue persuasively that focusing on protected areas neglects the political nature of the conservation process, and a lacks appreciation of the value-driven nature of biodiversity conservation. Maffi and Woodley (2010) corroborate this argument by contending that the debate of 'TEK vs. western science' is ill-founded, because the theories and practices which can lead to sustainable use of natural resources do not have to be comparable to those used in academic science. Further, they note that misconceived expectations of this sort can lead the further disenfranchising of local and indigenous peoples (Maffi and Woodley 2010). Similarly, Nadasdy (2005) argues that: "the debate over whether indigenous people are or are not ecologically noble is a spurious one, since it necessarily entails evaluating their behaviour according to imposed Euro-North American cultural assumptions. As a result, this whole debate serves to obscure rather than illuminate the complexities of the relationship between indigenous peoples and the environmental movement" ( $\mathrm{p}$ 322). Indeed, considerable progress has been made on the 'interfacing' of TEK and science with regards to natural resource management, and a variety of recent projects have shown that indigenous knowledge can contribute to scientific knowledge and vice versa (Stephenson and Moller 2009).

More fundamentally, however, scholars have disagreed with the delineation and conceptualisation of indigenous knowledge in opposition to western knowledge (Agrawal 1995; Agrawal 2009; Dove et al. 2007). Much of this disagreement stems from the difficulty of defining 'indigenous', which is not an intrinsic category in itself but is rather derived from modern political constructions of place and locality (Dove et al. 2007; Ellen and Harris 2000; Sundar 2002). Agrawal (1995) goes further, and labels the dichotomy between western and indigenous knowledge as 'ridiculous'. In this oft-cited article, the author notes that although indigenous and western knowledge have been separated on substantive, methodological, and contextual grounds, none of these arguments are robust. Therefore, he argues, “...it makes much more sense to talk about multiple domains and types of knowledge, with differing logics and epistemologies. It is something of a contradiction - though an unavoidable one - that the same knowledge can be classified one way or the other, depending on the interests it serves, the purpose for which it is harnessed, or the manner in which it is generated" ( $p 5)$. Other research has noted that, in practice, the two types of knowledge are difficult to delineate, and are constructed by various actors to suit various purposes over time (Dove 2000; Dove 2002). 


\subsubsection{Description of TEK in popular literature}

Similarly, scholars have argued that descriptions of TEK (and of indigenous people more broadly) in popular and academic literature have at times been over-simplified and distorted. Much of the popular interest in ethnobotanical knowledge of indigenous peoples has been driven by evocative descriptions by authors such as Wade Davis (Davis 1996; Davis 2009; Davis and Henley 1990) and Mark Plotkin (Plotkin 1993). These depictions cast indigenous peoples in a role as knowledgeable stewards of the land, imbued with ethic of sustainability and ethereal understanding, and often framed in opposition of the rapacious and reductionist 'western' forces of logging companies and scientists (Voeks 2004).

Commentators have argued that these descriptions have been driven by the need of environmentalists and indigenous activists to raise awareness, and funds, to their cause. To do this, these groups need to have a narrative that is recognisable and has value to the western audience, leading to a focus on over-simplified descriptions of indigenous peoples as stewards of pristine landscapes (Brosius 1997). For example, the anthropologist Peter Brosius (1997) describes the situation of Penan in Borneo, who were the subject of worldwide attention for their efforts to stop logging during the 1980's and 1990's. Brosius uses a variety of publications, in particular Davis and Henley (1990), to illustrate that in order to gain support and interest for indigenous peoples popular authors must appeal to the audience at hand. As he notes: “...to save something, or to mobilise an audience to want to save something, requires that it be made beautiful and profound, or to have some transcendent value. In creating that value, however, the thing itself is transformed" ( $p$ 67). Not only do these constructions impose false meanings onto TEK and indigenous peoples that may be quite imaginary, but it makes generic the very diversity that the arguments are trying to advance (Brosius 1997). Voeks (2004) notes similar phenomena regarding the medicinal pharmacopeia in the Amazon rainforest, where the construction of indigenous ethnomedical knowledge ignores the profoundly human altered nature of the landscape, as well as the high value of secondary and disturbed habitats for medicinal foraging (Stepp and Moerman 2001).

\subsubsection{Intellectual property, biopiracy, and TEK research}

Research into TEK and indigenous knowledge has remained a site of significant conflict, due to the threat of unauthorised use and exploitation of knowledge and genetic resources (Posey 2000; Posey and Dutfield 1996). In the past, the knowledge and practice of indigenous peoples have been appropriated for commercial gain in areas from pharmaceutical development (Cox 1999; Posey 2000) to adventure tourism (Regenvanu 2006). Intellectual Property Rights (IPR) are promoted by major international agreements, such as the Convention on Biological Diversity, as the primary mechanism 
for the equitable sharing of benefits stemming from commercial exploitation of TEK. However, IPR are problematic in the context of indigenous knowledge, for several reasons (more fully discused in Posey 2000: p 40). These include that they undermine the free and communal sharing of culturally held resources; that they are intended to benefit society through the granting of individual rights, which may not apply to collective entities; that they cannot protect information that does not result from an act of 'discovery'; and they do not mesh well with non-western systems of ownership, norms, and values.

Given the richness and importance of biological and cultural diversity in the Pacific, debates over biopiracy and IPR are of particular concern throughout the region (Marahere 2004). The issues have been given prominence by several issues over the past decade, including the patenting of kavalectone, an extract of kava, by German and US companies as a recreational and medicinal drug (Lindstrom 2009); and the success of AJ Hackett's bungy jumping operation in New Zealand and abroad, which is widely acknowledged as being based on the Nagol ceremony of Pentecost Island in Vanuatu (Regenvanu 2006). In both cases, acknowledgement of the original TEK holders has been limited, and designation of IPR remains contested (Lindstrom 2009). The draft 'Model Law for the Protection of Traditional Knowledge and Expressions of Culture' was developed by the Secretariat of the Pacific Community (SPC) in 2002, in an attempt by Pacific Island nations to protect their traditional knowledge and expressions of culture. However, it has yet to be converted into legislation in most Pacific states (Blakeney 2011).

Given the potential commercial value of TEK, research in the area of traditional knowledge is inextricably connected with issues of IPR. Specific concerns are often voiced about the valuable nature of ethnomedical knowledge to bioprospecting companies (e.g. Cox 1999); however issues with the regulation of the IPR of TEK permeate the full spectrum of cultural expression and practice (Regenvanu 2006; Marahere 2004). There are two key sites of concern. First, there are significant asymmetries of information and power between the research and informant, meaning that research participants often have little control over where their information goes and how it is utilised. Second, the nature of scientific publishing is such that knowledge, once released, can be obtained by anyone without the prior consent of the TEK holder (or, indeed, the researcher). This means that even research with benign intentions has potential to be used for commercial purposes. Preventing the misuse and appropriation of TEK research is a key concern of Human Ethics procedures worldwide, and underpins local efforts to regulate and control research, such as the Cultural Research Permit (CRP) in Vanuatu (discussed in Section 4.5). I acknowledge that these issues are relevant to my research, and outline my response to them in Section 3.3. 
More broadly, research into indigenous knowledge and TEK has been accused of maintaining neocolonialist agendas which perpetuate existing asymmetries and historical injustices (Smith 1999). Because western and scientific methodologies are deeply "...embedded in the multiple layers of imperial and colonial practices" (Smith 1999: p 2), they may be incapable of providing benefits to indigenous groups or of accurately describing the knowledge which they seek to research. Most researchers are fundamentally outsiders, and they can fail to recognise the lived nature of knowledge and the historical relationships which permeate the bodies of culture and knowledge (Holmes 1996, cited in Berkes 2008). Moreover, in seeking to describe knowledge, researchers intrinsically pass TEK and indigenous knowledge through their own 'cultural lens', and in so doing can fundamentally change the nature of the knowledge itself. A number of recent works have sought to address power imbalances within research agendas (see, for example, Crawford 2009; Smith 1999); and again, I discuss my own response to these critiques in Section 3.3 below.

\subsection{A resource at risk? TEK erosion and the problems of measurement}

I've shown above that our appreciation of the value of TEK has been enhanced by a number of research programs over the past three decades. The rise in academic valuations of TEK has come, however, at a time where we know TEK and cultural diversity to be increasingly at risk in the face of global homogenisation (Davis 2009). Indeed, a sense of urgency is now pervasive in most academic writing on the subject, as the magnitude of linguistic and cultural erosion becomes clear (Harmon 2002). For example, the ethnobotanist Paul Cox (2000) notes that whilst we are better positioned to understand the value of traditional knowledge, “... an increasing number of aged healers are dying, with their knowledge left unrecorded" ( $p$ 45). The imperative to document and maintain disappearing knowledge was one of the key initial drivers of this research program: whilst, as I will show, the on the ground reality is usually more complicated than such simple generalisations, it is nevertheless clear that the conservation and discussion of TEK is a critical area for future research.

Much of the concern surrounding TEK erosion is driven by the parlous state of the world's linguistic diversity. Although 6,909 languages currently spoken (Lewis 2009), ${ }^{2}$ the distribution of speakers is extremely uneven: the top ten largest languages account for over $50 \%$ of the population, whereas the 3,586 smallest languages are spoken by only $0.2 \%$ of humans (Harrison 2007). In total at least half our global stocks of linguistic diversity are expected to be extinct by the end of the century (Krauss

\footnotetext{
${ }^{2}$ Counting languages is a fraught task, and statistics such as these are constrained by two key factors. First, although the SIL Ethnologue database cited here gives the most widely-cited figures for global linguistic diversity, data are fairly unreliable and are often based on 'wildly uneven' sources, ranging from almanac citations to notes from field linguists (Harmon 2002). Second, because many languages in fact exist in a continuum of tongues, dividing up these into discrete entities is a purely socio-political (rather than scientific) enterprise (Harrison 2002; Muhlhausler 1996).
} 
1992; Krauss 2007), an extinction rate that dwarfs the better known crisis of biological diversity (Sutherland 2003). Moreover, the weight of socio-political imperialism and the momentum of cultural homogenisation is such that these trends will prove difficult to reverse (Muhlhausler 1996; Romaine 2008). Linguistic diversity is an imperfect proxy for cultural diversity more broadly, however it is the only measure for which sufficient data is available to estimate extinction rates (Harmon 2002). Using this information, there seems little doubt that many small indigenous languages (in which the bulk of TEK is encoded) are at extreme risk over coming generations.

A growing body of empirical, often quantitative, evidence has supported this hypothesis in a number of regions of the globe (Zent and Maffi 2009). This evidence has been based in a number of biogeographic contexts and amongst a wide variety of peoples (Zent and Maffi 2009). Authors have identified a number of factors as driving TEK erosion, both individually and interactively. These include religious conversion (Caniago and Siebert 1998; Voeks and Sercombe 2000), competition from Western medicine (Straume 2009; Ugent 2000), formal education (Boster 1986; Lizarralde 2001; Voeks and Leony 2004), economic integration (Benz et al. 2000; Brodt 2001; Voeks and Nyawa 2001) values change (Case et al. 2005; Estomba et al. 2006; Ohmagari and Berkes 1997), ecological changes (Ladio and Lozada 2004; Ross 2002a), and language shift (Harrison 2007; Maffi 2004; Nettle and Romaine 2000). In general, commentators refer to the 'seductive influence of modernisation and the western worldview' (Voeks and Leony 2004: S295). The prevalence of many of these factors worldwide has led the general sense of urgency that now pervades research in the area.

The erosion of TEK is likely to be having multiple impacts, given its importance in a number of socio-ecological contexts. Broadly, loss of TEK may be increasing incidences of 'cultural blind spots' that impede recognition of systemic problems (Muhlhausler 1995), and the loss of adaptive tools for coping with unforeseen environmental problems (Berkes et al. 2000; Maffi 2004). Detailed TEK, built through historical connection with the land, can impact on the way in which human groups perceive and manage the environment and is a key determinant of group-level ability to manage common-property resources (Atran 1999). Specific examples of the impacts of TEK loss include suggestions that erosion of TEK has been impacting on the functionality of reef management systems (Smith et al. 2007); eroding the ability of community members to be medicinally selfsufficient (Berlin 1999); and reducing the diversity (and thus the resilience) of agriculture in Brazil (Hanazaki et al. 2000). Other evidence shows that loss of traditional knowledge surrounding disaster management in the Pacific (such as the preservation of famine foods) may have increased vulnerability to extreme weather events (Campbell 1990; Campbell 2006). Moreover, there has been anecdotal evidence put forward that the erosion of TEK in the community context has contributed to social problems, including loss of respect for traditional values and community structures (Odochao et al. 2006). However, few studies have set out to directly quantify the impacts of TEK 
loss, probably due to the difficulty of unravelling the specific causal relationships between erosion of TEK and environmental or social problems (Berkes 2008).

\subsubsection{Measuring variation and erosion of TEK}

Given that TEK is likely to be at risk in a number of contexts, a key task of ethnobiological research is the assessment of TEK change and erosion. However, the erosion of TEK is a difficult process to empirically measure, largely due to a widespread lack of longitudinal time series data. In an ideal world, to measure change one would compare current levels of a particular indicator - say, knowledge of medicinal herbal plants - against baseline data, and check for significant difference. However, these data do not exist in most areas of the world. To account for the lack of longitudinal data, researchers have studied individual-level variation in TEK (Reyes-García et al. 2007a). Research of this kind draws from the field of cognitive anthropology, and is based on a definition of culture as shared knowledge (Romney et al. 1996). Using this definition, it has used a variety of techniques to measure intra-cultural variation (Reyes-García 2001). This research shows that knowledge is heterogeneously patterned within and between communities (Ghimire et al. 2004), and that this variation is associated with demographic characteristics such as gender (Boster 1986), kinship affiliation (ibid.), and age (Phillips and Gentry 1993).

Investigators have noted the variation of TEK in concert with a number of socio-demographic indicators. These have included age (Voeks 2004; Zent and Zent 2004), gender (Begossi et al. 2002; Case et al. 2005; Voeks 2007), birth locality (Voeks and Leony 2004), residence time (Nyhus et al. 2003), time spent in formal education (Quinlan and Quinlan 2007; Reyes-García et al. 2005), church attendance (Voeks 2004; Voeks and Sercombe 2000), linguistic ability (Benz et al. 2000; Reyes-García et al. 2005), rates of market visitation (Apaza et al. 2003; Reyes-García et al. 2005), and employment in a commercial occupation (Godoy et al. 1998; Godoy et al. 2005; Reyes-García et al. 2005). It is primarily these data which have been used to infer the erosion of TEK suggested in the paragraphs above.

Trends in the individual and community-level variation of TEK have been inconsistent across studies, however. While some research has noted strong age-related variation and have used this to infer loss of TEK over time (e.g. Voeks and Leony 2004), others have found no evidence of change (Byg and Balslev 2004; van Etten 2006; Zarger and Stepp 2004), or have even observed increases in TEK under certain economic conditions (Guest 2002). Similarly, some authors have found that formal education is implicated in the erosion of TEK (Quinlan and Quinlan 2007; Zent 2001), while others have noted that the number of years spent in formal education is positively associated with measures 
of ethnobotanical knowledge (Byg and Balslev 2004; Reyes-García et al. 2005; Reyes-García et al. 2007b). As Zent and Maffi (2009) observe after their review of the literature:

"...one of the main overall tendencies that stands out...is the dynamic complexity of TEK in space and time. At a local level, such complexity is marked by widespread sharing alongside internal variation, simultaneous continuity and change...the derivation of the corpus from endogenous as well as exogenous sources highlights the inherently fluid and shifting nature of TEK, which... [is] undergoing a continuous regenerative process encompassing a complex mix of replication, loss, addition, and transformation of constituent elements" ( $\mathrm{p} 39)$.

As such, change or erosion of TEK is an inherently difficult phenomenon to measure. Moreover, a number of methodological and conceptual difficulties have hampered interpretation of the trends in TEK variation.

One issue is the lack of time series data noted above. In order to infer erosion of TEK from the synchronous data available, researchers have typically followed three steps (Godoy et al. 2009). First, they have taken measurements of an indicator of TEK from people of different ages. Next, they have compared these measures between age cohorts (usually in concert with other socio-economic data). Finally, they have inferred change based on differences between older and younger age cohorts. This kind of study is useful, and if interpreted alongside the other common socio-economic explanatory variables mentioned above, can allow researchers to make some comment on the patterning of knowledge within the community at that time. However, estimating change based on age cohorts is often confounded by normal knowledge accumulation curves: that is, older people have had more time to assimilate knowledge about the environment than younger people, and as such we would expect them to know more. This effect can be examined by looking for non-linear trends between age cohorts (sensu Voeks and Leony 2004), however remains as a constraint of the study method.

Another methodological challenge is that TEK is cognitively broad, and is made up of a number of constituent domains (such as knowledge of medicinal plants, of animals, or of the marine environment). To date, most research on TEK variation has focussed on ethnobotanical knowledge, within the domains of useful plants (Byg and Balslev 2004; Casagrande 2002; Hanazaki et al. 2000), medicinal plants (Benz et al. 2000; Sternberg et al. 2001; Voeks and Leony 2004), wild food plants (Godoy et al. 1998; Zarger and Stepp 2004; Zent 2001), and crops (Boster 1986; Peroni and Hanazaki 2002; Salick et al. 1997; van Etten 2006). Other researchers have used cognitive methods (Atran et al. 2002; Boster 1986; Zent 2001) or objective tests (Godoy et al. 1998; Ross 2002b) to measure wider environmental knowledge. There remains very little research investigating TEK 
variation outside these domains (Reyes-García et al. 2007a; Zent and Maffi 2009). This is important, as the impacts of modernisation may impact on different domains of knowledge in diverse ways, and result in erosion of some domains and not others.

A third methodological challenge, as indicated by Figure 2.1, is that TEK consists of multiple dimensions including knowledge, practice, and belief. Some of these aspects of TEK are collectively held, whereas others are individual. Therefore, the successful study of variation or erosion across dimensions of knowledge requires the use of a range of methodologies. For example, while methods that focus on collecting individual responses may be appropriate for capturing the inner rings of the nested egg (i.e. empirical knowledge of plants and animals), other dimensions (such as the change in the worldview of participants), would be better captured by shared group techniques, such as Participatory Rural Appraisal methods (Kumar 2002). In practice, only a few studies have successfully differentiated between theoretical and practical dimensions of knowledge (i.e. intellectual ability versus practical skills), and have found that the two dimensions of TEK are not necessarily correlated (Byg and Balslev 2001; Ladio and Lozada 2004). Other research has focussed on providing a broader overview of TEK erosion by using a systems perspective (Brodt 2001). However, in general, empirical TEK research into the outer layers of the nested egg has remained minimal, and research has remained focussed on individual responses that deal with easily-described empirical knowledge and practice. ${ }^{3}$

Further, quantitative research into the variation of TEK thus far has shown a significant geographic skew. Of the 34 quantitative ethnobotanical studies reviewed by Reyes García et al. (2007a), all had been conducted in developing countries. Of these, 70\% took place in Latin America, 21\% in Africa, and $9 \%$ in Asia. The authors of that study note that they find no studies from North America, Europe, and implicitly (though this was not noted), none from Oceania. This was ascribed to the fact that much of the research attention in the field has come from a limited number of research programs, including that of Reyes-García et al. in Bolivia, Begossi et al. in Brazil, and Atran et al. in Central America and Mexico. Clearly, processes of TEK erosion and change are likely to be locally specific, as drivers will differ in nature and importance at local, regional, and national levels. Therefore, results from one area of the world may not be generalisable to another.

In summary, there is little doubt that TEK is under threat. However, most of the evidence produced thus far provides a compelling illustration of the complexity of any changes that are taking place, and offers proof (if any were needed) that processes of globalisation and homogenisation are never

\footnotetext{
${ }^{3}$ I'd note here that the apparent lack of research attention may be indicative of my focus on the ethnobiological literature, as there as there are large literatures on cultural change within, for example, sociology (e.g. Inglehart and Baker 2000) and the evolution of social institutions for resource management (e.g. Ostrom 1990). These are, however, outside the scope of this literature review (see Section 3.5 for a discussion of the limitations of this thesis).
} 
straightforward (Quinlan and Quinlan 2007). Our understanding of TEK change or erosion is further hampered by the methodological dissonance and conceptual difficulties noted above, and by the geographic skew which precludes our understanding of these processes in Oceania.

\subsection{Situating TEK: Cultural transmission}

The literature outlined in Section 2.4.1 has increased our understanding of the ways in which TEK is patterned within and between communities. However, such analyses are able to say very little about how the TEK holders in their research came to acquire their knowledge (Reyes-García et al. 2009). This is a critical issue because TEK, as with all facets of culture, is perpetuated and sustained by processes of cultural transmission (Ohmagari and Berkes 1997). If we do not understand the ways in which actors in a social network acquire and disburse information, we are unable to comment on the mechanisms that influence patterns of variation (Ross 2002a; Zarger and Stepp 2004). Importantly, we are also unable to comment on the processes that may be driving change or erosion in TEK (Cristancho and Vining 2009). Therefore, the cultural transmission of TEK should be a critical target for future research. As yet, however, it remains under-represented in the ethnobiological literature (Zent 1999).

I address this literature gap using mixed-methods research in Chapter Five. The cultural transmission of TEK is extensively reviewed in this chapter (Section 5.1), so will not be presented here.

\subsection{Revitalising culture: TEK maintenance}

The literature reviewed so far indicates that TEK is both important and at risk. This has led to a veritable " ... outpouring of eminent scholarly opinion declaring the study and preservation of [TEK] to be a most urgent task of great scientific, economic, and humanitarian value" (Zent 1999: p 91). The same author has proposed an 'ethnoecological imperative', in which refers to the urgent need to document and conserve traditional knowledge and management systems before they are gone forever (Zent 1999: p 91), a sentiment echoed in a number of other works (for example Harmon 2002; Harrison 2007; Maffi 2004). Others, such as Heckler (2002), have noted that approaches to TEK maintenance should focus on 'innovative' strategies, which should be urgently put in place in order to ameliorate the ongoing erosion of TEK. The imperative to conserve and maintain systems of TEK has been driven by several international agreements, including the Convention on Biological Diversity (Article 8(j): see Posey 2000) and the United Nations Declaration on Indigenous Peoples (Articles 11 and 31; see Barelli 2010). There has been, however, surprisingly little evidence and discussion in the ethnobiological literature surrounding the maintenance of TEK, a literature gap 
which is in need of filling (Pilgrim et al. 2007; Pretty 2003; Zent 1999). The literature that does exist has focussed on three primary methods for the conservation of TEK: ex situ approaches, the formal school, and in situ approaches. These are detailed below.

\subsubsection{Ex situ TEK maintenance}

One group of authors has advocated the recording and dissemination of TEK in databases (Warren 1995; Warren 2001; Warren et al. 1995). This has variously been termed 'ex situ' or 'extractive' TEK conservation (Agrawal 1995; Zent 1999). These approaches typically seek to record ethnobiological knowledge and to store it away from its source culture, often in online databases (e.g. Rahmann 2000; TKDL 2011; UNESCO 2011; World Intellectual Property Organisation 2011). Ex situ TEK conservation programs cite a variety of motivations, from aiming to harness the economic potential of ethnobiological knowledge to inspire products of technological and commercial value (e.g. the Korean Traditional Knowledge Portal 2011), to seeking to broadly share successful local adaptations and further goals of resilience and sustainability (Gadgil et al. 2000; Plants for a Future 2011; UNFCCC 2011). The latter motivation is guided by the ideology that “... by recording knowledge, and making it available to the global community... community-based systems will in the near future begin to be regarded as contributions to global knowledge" (Warren 1995: p 32). Other organisations, such as the World Intellectual Property Organisation (WIPO), maintain databases of ethnobiological and other knowledge so as to facilitate the study of the application of intellectual property rights to traditional knowledge and practice.

Ex situ approaches face two common criticisms. First, it has been contended that they may “...overlook the culture- and environment-specific nature of ethnoecological knowledge, the information content of such knowledge being necessarily altered and distorted by removing it from its original cultural-ecological context" (Zent 1999: 93). If traditional knowledge gains value from being part of lived, everyday experience, then there is little point in removing and isolating it from the source culture. Moreover, such approaches negate the dynamic and fluid nature of ethnobiological knowledge (Agrawal 1995). Second, such approaches raise serious questions about intellectual property and access to knowledge, as it can easily lead to the private appropriation and commercial exclusion of the knowledge from the source communities (Agrawal 1995).

Recent technological advances may go some way towards adjusting these dynamics. For example, in a long-running program at Marovo Lagoon in the Solomon Islands, researchers have been utilising widespread wireless broadband internet and cheap laptops to create a locally run Wikipedia-type encyclopaedia of TEK (Hae Rokona 2011; Hviding 2005; Tuiono 2011). Community-members have been trained in updating and checking the database, with the underlying purpose that this technique 
both empowers locals and conserves TEK in a secure database. However, technological innovations (such as the storage and dissemination of TEK on CDs and books) have been argued to fundamentally misunderstand the nature of knowledge transmission in traditional societies (Bates 2009). Moreover, the use of technology potentially increases reliance on outside help and diminishes the importance of traditional modes of knowledge transmission.

\subsubsection{TEK and formal education}

The second approach to TEK conservation which has been mooted in the ethnobiological literature is the inclusion of TEK in the school curriculum (Heckler 2002; Reyes-García et al. 2005; Zent 1999). The integration of TEK into the formal school curricula has been suggested as potentially having a number of benefits, and may maintain TEK by utilising the infrastructure and reach of formal school systems (Reyes-García et al. 2005); by empowering local knowledge and placing it on the same standing as that of western or non-indigenous knowledge (Kimmerer 2002); and by facilitating new structures for intergenerational knowledge transmission where older social networks are no longer functional (Aikenhead and Ogawa 2007).

Such moves may mitigate some of the negative effects that formal schooling has been shown to have on TEK. As noted above, there may also be distinct educational benefits from contextualising education curricula with local content, including the promotion of pupil self-esteem and identity and improved comprehension through the connection of abstract concepts with local environments and realities (Brayboy and Castagno 2009; Castagno and Brayboy 2008). Moreover, the teaching of TEK in school is likely to facilitate learning from the environment and from the land, thus mitigating the 'acquisition deprivation' that comes from separation from the environment and facilitating the development of pro-environmental behaviour (Bates 2009; Hunn 2002).

It is worth noting that these debates, largely confined to the ethnobiology literature, run parallel to a large body of work within the field of education. Especially within the Pacific, educators have noted the need for localisation of school curricula, which are typically based on Western models that are argued to bear little relevance to daily life (Pene et al. 2002; Sanga et al. 2004; Teasdale et al. 2005; Thaman 2000; Thaman 2002). Proponents argue that education should be a process of 'worthwhile' learning, which passes the skills of daily life on to pupils, and note that "...the failure of education in the Pacific can be attributed in large measure to the imposition of an alien system designed for Western social and cultural contexts, which are underpinned by quite different values" (Taufe'ulungaki 2002: p 15). More broadly, there is a vigorous debate around the merits of 'universalist' versus 'multiculturalist' positions in science education, with the former party arguing for the universal application of science, and the latter party arguing for appropriate recognition of the 
diversity of ways of knowing and learning (Matthews 1994; Siegel 2002; Sillitoe 2007; Snively and Corsiglia 2001; Stanley and Brickhouse 2001). Multiculturalists have argued that the universal application of western modes of knowing and knowledge are akin to forced acculturation (Aikenhead 2001; De Beer and Whitlock 2009), and have gone as far to claim that there is 'systemic racism' present in science curricula worldwide (Aikenhead 1993 p: 659).

These discussions have been reflected in an evolving body of practice and policy with regards to curriculum reform (Teasdale 2005). A number of countries, particularly within the Asia-Pacific region, have begun re-orientating their curriculum around localised and contextualised educational objectives (Bajunid 2008; Maclean 2002; Teasdale et al. 2005). For example, in Vanuatu senior figures at the national Ministry of Education have noted that "...it appears that the time is here for the cultivation of the local agenda, [local] interpretations, and man ples [local] perspectives" (Sanga and Niroa 2004 p: 15), and a recent Curriculum Statement admits that "...the current curriculum is not designed for Vanuatu's diverse cultures and communities... our national curriculum is in need of reform so it reflects our national identity"(Vanuatu Ministry of Education 2010 p: 2). One key driver of this shift has been the publication of a keystone UNESCO document (Delors 1996), which suggested education policy is revised around four 'pillars': learning to know, learning to do, learning to live together, and learning to be. Whilst the western model of teacher-centred learning traditionally focuses on the first two, Jacques Delors and his Commission accorded equal importance to latter two pillars, emphasising the need for students to be aware of their history and place in the world. Although implementation of the goals of the Delors Report (in particular, the recognition of the latter two pillars) has been difficult (Delors 2002), the high-level recognition that local knowledge has value in education has legitimated the inclusion of indigenous knowledge in school, and has provided fertile ground for discussion about the use of education systems for TEK maintenance (Teasdale et al. 2005).

That said, proposals for the integration of TEK in formal school curricula remain controversial. Some have argued that such moves risk de-contextualising TEK by forcing it to be transmitted in a fundamentally different manner to 'traditional' forms of knowledge transmission (Bates 2009; Shukla and Gardner n.d.). Others have noted that widespread arguments for the insertion of local perspectives in formal education risk replacing 'one form of colonial mindset with another', by oversimplifying the dynamic and diverse nature of culture (Burnett 2007; Burnett 2009). Moreover, such moves may do little to address the underlying power imbalances which drive discrimination against local and indigenous knowledge in the first place. Sundar (2002) observes that “...the valorisation of a certain body of knowledge as 'indigenous' and its incorporation into a formal education system depends on the status and power of the social group claiming 'indigenous' status, rather than the substantive content of the knowledge. Indeed, the distinction between 'indigenous' 
and 'non-indigenous' is particularly invidious when it comes to knowledge, masking the imbrication of all forms of knowledge in particular regimes of truth and power" ( $p 373$ ).

\subsubsection{In situ TEK maintenance}

The third key approach to TEK revitalisation is the promotion of in situ approaches to TEK maintenance. Broadly defined, in situ approaches seeks to maintain and revitalize knowledge and practice within the source community, as opposed to extracting it for storage in databases or use in state-sponsored initiatives such as formal schools (Agrawal 1995). Such approaches may be more cost effective than the approaches outlined above, as a result of being based within the source communities and thus requiring limited infrastructure (Pilgrim et al. 2009). Moreover, they may be more sustainable than ex situ and centralised TEK conservation, as they are rooted in local power structures and are able to be responsive to local needs and concerns. In situ approaches to knowledge conservation may also have wider benefits that accompany cultural conservation, such as facilitating sustainable resource management (Pilgrim et al. 2009), or promoting the conservation of indigenous crop diversity (Altieri and Merrick 1987; Louette et al. 1997).

More broadly, in situ approaches allow for holistic conservation of the institutions and 'cultural landscape' of the TEK holders (Agrawal 1995). As such, they are akin to programs which seek to maintain and revitalise vernacular languages through the recognition of 'linguistic ecology' (Florey 2009; Haugen 1972; Muhlhausler 1996). Such programs recognise that “...ecological factors bring language into being, define their boundaries, and decide their growth and survival" (Muhlhausler 1996: p 3), and so recommend the deployment of bottom-up strategies that serve to maintain the entire cultural framework of the language (Florey 2009).

Similarly, the conservation of discrete domains of TEK (such as ethnomedical knowledge) independent of the cultural context in which that knowledge has evolved is likely to fundamentally change the TEK itself. In situ approaches, in placing control for the conservation of knowledge in the hands of the communities themselves, may address this deficiency (Agrawal 1995). More fundamentally, however, approaches such as this may have potential to address the root causes of loss of traditional knowledge and vernacular language by empowering the indigenous and local people who hold it. As noted by Agrawal (1995): "If indigenous knowledge systems are disappearing, it is primarily because the pressures of modernisation and cultural homogenisation, under the auspices of the modern nation-state and the international trade system, threaten the lifestyle, practices, and cultures of nomadic populations, small agricultural producers, and indigenous peoples. The appropriate response from those who are interested in preserving the diversity of different knowledge systems, might then lie in attempting to reorient and reverse state policies to permit 
members of threatened populations to determine their own future, thus facilitating the in situ preservation of indigenous knowledge" (p 5). Successful in situ conservation of TEK is, then, a question of addressing power imbalances that are usually present between state actors and minority groups who hold the TEK. Of course, this is no small task, and is likely to pose significant political and ethical challenges (Agrawal 1995).

In situ TEK conservation approaches have taken a number of forms, however published accounts are relatively rare. One group of approaches aims to conserve both biological and cultural diversity through promoting the self-determination of indigenous and local peoples and raising the status of TEK and vernacular language (see Maffi and Woodley 2010). One example of this has been to focus on guaranteeing the natural resource base of TEK holders by granting land titles or establishing reserves, such as through the UNESCO Man Biosphere program (for example, see Dessisa in Maffi and Woodley 2010). Similarly, some approaches have sought to maximise the self determination of indigenous peoples and other TEK holders on their own land, in order to increase the relevance and transmission of TEK (for example, see Barnard and DeMarco in Maffi and Woodley 2010). Other, related, programs have sought to legally enshrine TEK and TEK systems in legal systems, such as the use of traditional resource management approaches in Marine Protected Areas (Aswani et al. 2007). Programs such as these have good potential to increase the transmission of TEK, and thus contribute to its maintenance. However, at least one scholar has argued that they may focus on incorrect solutions to the problem of TEK conservation, as they are often not based on a detailed understanding of knowledge loss, and thus cannot account for context-specific drivers of cultural change (Zent 1999). As such, they may be more "...concerned with conserving the necessary conditions for knowledge survival rather than the sufficient conditions” (Zent 1999: 94).

Another group of in situ TEK conservation approaches aim to directly facilitate the intergenerational transmission of TEK within the source communities. These approaches may sidestep Zent's criticism above, however may also risk not addressing the underlying causes of knowledge erosion (Agrawal 1995). Pilgrim et al. (2009) outline a number of responses that fall into this grouping, including culturally-appropriate education systems (with examples from Canada, Alaska, and Australia) and programs for the revitalisation of traditional food systems and knowledge (with examples from Greenland, South Africa, and Russia). Other good examples have been documented, including: Aikenhead (2001), discussing the formation of a 'Rekindling Traditions' project in Northern Canada for reconnecting elder and younger generations; Plotkin (1993), in a popular book describing the 'Shaman's Apprentice' program; or a variety of accounts from India, documenting efforts at the recognition and maintenance of TEK in various community contexts (Brodt 2001; Gadgil et al. 2000; Singh et al. 2010). Authors have also suggested the implementation of 'bush camps' for the teaching of TEK in direct proximity to the environment, which would facilitate the 
transmission of land-based knowledge such as the ability to hunt and track wildlife (Bates 2009; Ohmagari and Berkes 1997).

\subsection{Chapter summary: Literature gaps}

In this chapter I have defined TEK and discussed some of the key controversies around the way it is researched and represented. I have also reviewed literature which indicates that TEK is a critical resource in many areas of the world, but that it is at risk of erosion. I then highlighted the difficulty of inferring change and erosion in TEK, briefly noted the lack of research investigating the cultural transmission of TEK, and discussed three key options for the maintenance and revitalisation of TEK.

Four key literature gaps emerge from the literature review:

1. Variation of TEK (Section 2.4.1): Research into the drivers of variation in TEK has thus far produced mixed and conflicting results. This may be partially due to methodologies which do not consider multiple domains and dimensions of TEK. Moreover, little research of this kind has been based in Oceania, despite its status as a hotspot of biocultural diversity.

- Literature gap: Broad-based research is needed into the drivers of TEK variation, especially in the Pacific region. Forms objective one, addressed in Chapter Five.

2. Cultural transmission of TEK (Section 2.5): Processes of cultural transmission govern the persistence or erosion of TEK, but have rarely been researched within ethnobiology. Moreover, changes in systems of cultural transmission are poorly understood. Quantitative and qualitative approaches often yield different results.

- Literature gap: Mixed methods research into the cultural transmission of TEK is needed. Research should utilise quantitative and qualitative methodologies and examine how systems of cultural transmission can change. Forms objective two, addressed in Chapter Six

3. TEK and formal education (Section 2.6.2): The introduction of TEK into formal school curricula may be able to add value to education and maintain TEK. However little research has examined perceptions of the validity of this approach, despite the potential for negative consequences 
- Literature gap: Qualitative research that examines the value of TEK to formal school curricula, and the ability of formal education to maintain TEK, is needed. Forms objective 3 (a), addressed in Chapter Seven.

4. In situ maintenance of TEK (Section 2.6.3): In situ maintenance of TEK may present the best option for the maintenance of TEK, but little is known of the factors which help or hinder the establishment of these schemes.

- $\quad$ Literature gap: Research is needed into the triggers and barriers to in situ TEK maintenance, which may then be used to discuss the merits and flaws of in situ approaches. Forms objective 3(b), addressed in Chapter Eight. 


\section{Methodology}

In this chapter I outline my methodological approach to addressing the objectives of this thesis. The approach and issues outlined herein underpin each of the core chapters, and impact on the conclusions I am able to derive from the data. I begin by describing my positioning regarding the research process and reflecting on how that may have influenced the nature and outputs of this research. I also note several key concerns regarding power dynamics and research into indigenous knowledge. Next, I outline my approach to accounting for these critiques, which involve rigorous ethical procedures and collaborative research design. I then briefly note the key methods used in this research, while leaving in-depth description to the relevant chapters. Finally, I define the scope of this research by discussing the limits of my approach, and briefly consider how this may impact on my findings.

\subsection{Philosophical assumptions}

I acknowledge that my worldview has fundamentally impacted on the nature of this dissertation (Guba and Lincoln 2005). In this section I explain my positioning with regards to the research process, the philosophical paradigm under which this research has been conducted, and discuss some of its key methodological assumptions, particularly regarding my approach to indigenous knowledge and to gender issues.

\subsubsection{Positionality}

I am an English-born male New Zealander, trained in the academy and scientific tradition through comprehensive secondary and tertiary education (focused on biological and social sciences). The social and political norms and values that have characterised my upbringing have fundamentally shaped and influenced the outputs and outcomes of this dissertation. Broadly, I see myself as having elements of both the 'bioenvironmentalist' and 'social green' worldviews, as defined by Clapp and Dauvergne (2005). The former group stress the biological limits of the earth to support economic growth, and oppose economic globalisation on the grounds that it has negative environmental impacts. I do not agree, however, that overpopulation is the key driver of environmental issues; 
rather, I'd argue that vastly unequal patterns of consumption are to blame. The latter group argue that economic globalisation serves to exacerbate inequality and reinforces the domination of the global plutocratic and patriarchal elite. As such, social greens advocate major reforms to the status quo, including the strengthening of community autonomy and the promotion of local and indigenous knowledge systems (Clapp and Dauvergne 2005). In addition, I stress my belief of the fundamental and inextricable links between social and ecological systems (or, if you will, between biological and cultural diversity), which positions human beings as one component within the larger ecosystem (Folke et al. 2007). These perspectives have fundamentally shaped this research, which is grounded in the assertion that epistemological diversity has an immense intrinsic value. In terms of my research methodology I work within the status quo, and hope to use established methodologies and approaches to increase the prevalence of work of this type.

\subsubsection{Pragmatist research}

I took a pragmatist methodological approach to this research (Creswell and Plano Clark 2007; Morgan 2007). Pragmatist research is rooted in the approaches of early theorists such as William James and James Dewey, and has recently grown in popularity due to theoretical and epistemological difficulties with the metaphysical research paradigm (Morgan 2007). Pragmatist research is based on a principle of 'workability' and a focus on the most practical and appropriate methods for answering the objectives at hand (Cresswell and Plano Clark 2007). As such, it is closely linked with mixedmethods and interdisciplinary work such as this, and emphasises that outputs gleaned from different research paradigms or approaches (such as constructivism and positivism) are often commensurable (Morgan 2007).

Philosophically, pragmatist research accepts multiple positions on axiology (values) within research, such as including both biased and unbiased perspectives; a relativist ontological perspective that recognises singular and multiple realities; and thus a diverse epistemological basis that can recognise the value of both objectivist and subjectivist viewpoints (or, as phrased by Morgan 2007, 'intersubjectivity'). Therefore this research approach, while accepting the need for awareness of the epistemological issues that shape our response to research problems, is critical of any knowledge of notion of knowledges in absolute (Creswell and Plano Clark 2007). This makes it an ideal approach to take to research that deals with indigenous knowledge and practice, which may be based in different epistemological frameworks (Cresswell and Plano Clark 2007).

I note that Chapter Five and elements of Chapter Six have elements of a positivist approach to research. These include a deductive, quantitative framing of the research questions and focus on empirical enquiry. These approaches are reductionist in nature and seek to describe TEK and TEK 
transmission in quantitative and abstract terms, in order to verify theories regarding processes and dynamics of knowledge change. That said, the focus of the overall research program is on the outcomes and consequences of enquiry (rather than the antecedent conditions as in positivism) and so fit into the largely pragmatic approach of the research (Creswell and Plano Clark 2007).

More broadly, I follow Lancy (1996) and position myself between nomothetic (universalist) and ideographic (particularist) perspectives. The former (such as neoclassical economists and sociobiologists) premise their investigations on the uniformities of structure and content in human life. The latter (such as cultural phenomenologists) emphasise the uniqueness of culture and tend to reject transcultural categories and comparative methods (LeVine 1984). I believe that the variation and diversity that characterises Vanuatu is great, and profoundly influences processes of cultural change and the persistence of ethnobiological knowledge. The processes described herein are unique to the study sites, and derive from a particular set of processes being interpreted through a unique cultural lens. At the same time, the variation in these processes does not result in an infinite variety of patterns, and I believe that the theoretical insights gleaned provide grounds for robust speculation with regards to other contexts and spaces (Lancy 1996).

\subsection{Cross-cultural research}

The philosophy and positionality noted above have influenced the nature of this dissertation and the research described herein. In particular, my positioning as a western, male, researcher, working crossculturally in the area of indigenous knowledge, should rightfully attract scrutiny. In recent years, research within the social sciences has become increasingly critical, interpretative, and reflective on the process of research and documentation, with the result that we are considerably more aware of the implications of research (Guba and Lincoln 2005). There are several powerful critiques of research of the type that I have undertaken, which I briefly outline and attempt to respond to below.

Scholars have acknowledged the power dynamics that fundamentally shape the nature of fieldwork and research in the cross-cultural setting, to the extent of noting that relationships are "...always structurally weighted on the side of the researcher, who is implicated in Western colonialism" (Marcus 1997, cited in Bolton 1999a: p 3). Commentators have also rejected the notion of 'benign research', by observing that "...research is not just a highly moral and civilised search for knowledge; it is a set of very human activities that reproduce social relations of power" (Smith 2005: p 88). Therefore, fieldwork and research relations are fundamentally infused with issues of appropriation, representation and misrepresentation. This is often enacted both through the act of 'research' (such as talking to people), but also through the ways in which this data is represented after the fact, a process that is usually controlled by the researcher and which is often characterised by a fundamental 
lack of power on the part of the researched. This is a critical dynamic, as observed by Agrawal (2002) who "...insist[s] on the need to keep in the foreground the ways in which power works. Without explicit and continuing attention to how power structures knowledge, it will remain impossible to achieve the aim of working in the interests of indigenous or other marginal peoples" ( $p$ 295). The growing acknowledgement within academia of these fundamental power imbalances has led to some researchers to cease work in cross-cultural contexts altogether, on the basis that they have no social claim to answer the questions posed by the research process (Scheyvens and Storey 2003).

As already noted in Section 2.3.3 above, the ways in which research has been conducted with (or into) indigenous peoples and indigenous knowledge have been a site of severe criticism (Bishop 2005; Crawford 2009; Smith 1999; Smith 2005). Because the “...history of research from many indigenous perspectives is so deeply embedded in colonisation..." (Smith 2005: 87), and because the present realities of research are so implicated by uneven dynamics of power, there is widespread scepticism at the goals of cross-cultural researchers (Scheyvens and Storey 2003). As England (1994) observes, it is extremely problematic to incorporate the voices of the 'others' without colonising them in a manner that reinforces patterns of domination, an issue which is difficult to resolve given the fundamentally etic nature of most academic research questions (Scheyvens and Storey 2003). Scholars have argued that even when terms such as co-researcher or participant are substituted for the standard anthropological 'informant', they merely serve to obscure the fundamentally exploitative nature of the research relationship (Bolton 1999a). The result, in the New Zealand context, has been "...the development of a tradition of research into Māori people's lives that addresses the concerns and interests of the predominantly non-Māori researchers' own making, as defined and held accountable in the researchers' own cultural worldview(s)" (Bishop 2005: p 110).

Power dynamics are also critically important with regards to gender in research (Bernard 1998). Foreign, particularly male, researchers are often limited in their ability to use research to reveal the perceptions or thoughts of the opposite gender (Scheyvens and Storey 2003). This may be partially related to practical constraints, such as the fact that women in many places are extremely busy, and time to sit and talk may be restricted to evenings where it may not be possible, or appropriate. The ability of women to participate in the research process may be further constrained by social norms, by a lack of self-esteem on the part of women, or by the sensitive nature of the subject area (Scheyvens and Storey 2003). As Keesing (1985) comments:

"What women can and will say about their lives also reflects the politics of the elicitation situation-both in relation to male control over the ethnographic process and in relation to the values and pressures that shape women's self-conceptions. Reflective autobiography is possible only when subjects believe that their own lives are important enough to 
deserve recounting, and when social support is provided...if a people's dominant ideologies, expressions of male political hegemony, define what women know and do as secondary and unimportant, then creating a context where women can and will talk about themselves and their partly separate realms of life and expertise may indeed be difficult or ultimately impossible" (p 37).

This is particularly important with regards to TEK and indigenous knowledge, which is often structured by gender and changes or adapts in gender-specific ways (Andresen 2001; Briggs and Sharp 2004; Shiva 1988; Voeks 2007). Such patterning of knowledge has often not been reflected in research or research methodologies, in which women often remain under-represented or invisible. This may be partially due to the methodological constraints noted above (Agarwal 1992; Rocheleau 1991).

\subsection{Ethical approach}

\subsubsection{Addressing the critiques}

The critiques outlined above have the potential to undermine the validity of this research as a whole. In response, I argue that there is an intrinsic importance of this research program, and as such have attempted where possible to ameliorate and recognise the power differentials noted above. Of course, my starting point - that research into TEK is valuable in and of itself - could be questioned, and potentially will be by some. However I believe in light of the literature discussed in Section 2.2, and my own belief in the value of cultural diversity (as well as my assumption that this research can effectively address some of the key issues), this research area is important; as such, I do not spend further time justifying the underlying premise of the work.

It's worth noting that although the power dynamics noted above are important in shaping the nature and outcomes of the research process, it should not be assumed from the outset that research participants are passive and powerless, actors in a narrative driven entirely by the researcher (Scheyvens and Storey 2003). Participants can, for example, exercise control over the research process by refusing to answer questions, or (in group interviews) by discussing answers in the local vernacular language before answering back in a language understood by the researcher. Indeed, this was often the case during my work in Vanuatu: participants would sometimes explicitly note areas of discussion that were not available for conversation, or would simply refuse to be interviewed further on particular themes. By the same token, women would often decline to be interviewed. As noted by the anthropologist Peter Metcalf (2002): “...there is nothing self-evident about why anyone would bother talking to a would-be ethnographer - assuming they do - except perhaps for polite platitudes. 
In unfamiliar surroundings, he or she is usually socially inept, and often linguistically incompetent. Such people are usually avoided...we [also] have to consider the conceptual premises and conversational constraints under which they select what to tell and how to tell it" (p 1).

Nonetheless, the critiques noted above are important: even if there was no power differential in the gathering of data (which is unlikely to be the case), the information gathered still has to be repackaged so as to be relevant to an academic audience in products such as this dissertation. This raises two fundamental problems. The first is the inherent difficulty in describing knowledge that is not lived, and interpreting different epistemological positions such as those of my informants through the lens I described in Section 3.1 above (Brosius 1997). I deal with this issue through my pragmatist approach to research, which recognises multiple ontological, axiological, and epistemological positions regarding knowledge; and through my research design, which steers away from descriptive accounts of ni-Vanuatu TEK for precisely this reason.

The second key problem is that TEK, as understood and lived by the informants in this work, is inherently decontextualised and uprooted by the process of recording knowledge in this thesis. By nature this disempowers the original knowledge holders, as the products of this research will largely be outside their sphere of influence (Briggs and Sharp 2004). I attempted to address this issue through strict adherence to carefully constructed ethical guidelines, which spanned the research process from design to the production of outputs.

The ethical guidelines for this research were adapted from the Code of Ethics developed by the International Society of Ethnobiology (ISE: International Society of Ethnobiology 2006). Based on the founding principles of the Society and the 1988 Declaration of Belem (International Society of Ethnobiology 1988), the Code of Ethics was developed over a long period with a high degree of indigenous participation (Smith 2005). It is based on 15 principles on which ethical conduct rests, including principles of self-determination, traditional guardianship, and active participation.

Moreover, the Code of Ethics suggests that research be built on principles of meaningful partnership and collaboration with indigenous communities.

All procedures used in this research program were approved by the Human Ethics Committee (HEC) at Victoria University. This research was approved in two phases, once for the Masters/scoping section of the work in 2008 (approval number 15452; Appendix 11.1), and subsequently for the remainder of the research program in 2009 (approval number 16500; Appendices 11.3 and 11.4). This process assures that all research being undertaken by Victoria University scholars is congruent with the Human Ethics Policy ensures the rights of individuals and groups who are the subjects of research (Victoria University of Wellington 2009). 
The ethical approach of this research also benefited from the detailed institutional infrastructure that exists within Vanuatu to ensure indigenous control over the activities of foreign researchers (Regenvanu 2005; Regenvanu 2006; Regenvanu 2007a). All cultural research in Vanuatu must gain approval from the National Cultural Council, who issue an annual quota of Cultural Research Permits to foreign researchers (Vanuatu Cultural Centre 2011). Their Cultural Research Policy (CRP) is implemented by the Vanuatu Cultural Centre (VCC) under legislated powers, and since its inception in 1995 has provided an effective tool for ensuring local participation in research activities (Regenvanu 2006). Its provisions include requirements for local community approval of the research project: maximum involvement of community members in the research process; full recognition of collaboration with community members; training for community members in research documentation; provision of a cultural product of immediate benefit to the community; deposit of all copies of research products with the VCC and community; provision of a service of benefit to the nation; and respect for traditional copyright protocols (Regenvanu 2006). Again, my research gained approval from the National Cultural Council twice, first in 2008 for the Masters/scoping phase, and again in 2009 for the remainder of the $\mathrm{PhD}$ work.

\subsubsection{Ethics procedure for this research}

In this research I undertook a number of procedures to ensure that the research met the ethical standards of the VCC, the HEC, and the ISE's Code of Ethics.

Input from VCC staff at research design: Key VCC staff in Port Vila and on Malekula assisted with the design of this research by offering comments on overall direction, the appropriateness and salience of interview questions, and assisting with the design of community outputs. As noted, this research was granted a Cultural Research Permit by the National Cultural Council.

Free Prior Informed Consent: All interviewees were asked for their consent to the interview process, after being thoroughly briefed in Bislama on the aims, methods, and products of the research. Literate participants (officials in Port Vila sampled during research toward Chapter Seven) were asked to sign consent forms. Non-literate participants, including all interviewees from communities on Malekula, were asked for their verbal consent to the key questions on the informed consent forms. Information and informed consent forms are included in Appendices 11.2 and 11.5.

Confidentiality of participants: All participants in this research remain confidential

Confidentiality of data: Because of the intellectual property concerns surrounding biopiracy and the use of medicinal plant data (see Section 2.3.3), all medicinal plant data remain confidential. Therefore, I will not publish the binomial species names of any of the plants cited by informants during this 
research. This chiefly pertains to the research conducted toward Chapter Five. I note that scientific plant names are included in ethnobotanical resource books for use in the communities, however after consultation with the VCC and community members it was decided not to include specific use data.

Work in close contact with fieldworkers: The VCC is represented in the study communities by a network of volunteer 'fieldworkers' (see Section 0 below) who are trained in the maintenance and recording of traditional knowledge and vernacular language. They were gatekeeper informants in each community, and were available to advise on protocol, check the appropriateness of interview questions, and assist in sample selection.

Work with local female research assistants: Because of the concerns surrounding research with women identified above, female research assistants were employed to conduct structured interviews with all female informants. The research assistants were selected in consultation with the fieldworker in each community and were thoroughly trained. I was not present for any interviews they conducted, except those used for training purposes.

Payment for key experts: All participants who contributed significant amounts of time (over 3 hours input) to this research were paid. This included research assistants, botanical experts, translators, and the VCC fieldworkers. The rate of pay was set by the VCC at 1000 vatu (around NZD \$15.00) per day, or part thereof.

Outputs returned to communities: Products of this research were of direct benefit to communities. Most importantly, I designed four ethnobotanical resource books for use in teaching in the community (Appendix 11.13). These were designed in consultation with the VCC fieldworkers and other key experts in each community, and consisted of photo identification, lists of key species, and a short amount of text. A summary of the competed thesis, in Bislama, will also be sent to communities.

Outputs returned to Vanuatu: All research outputs, including published papers and the final thesis, will be returned to Vanuatu to be stored in the National Library in Port Vila. I will also write a short article for publication in the national newspaper (the Daily Post) on the findings of my research.

\subsubsection{Strengths and weaknesses of ethical approach}

These undertakings sought to address the concerns regarding power dynamics noted above. I attempted to minimise the power differentials between myself and interviewees through the use of informed consent procedures and the heavy involvement of local and VCC representatives. I have produced a wide range of outputs that include some of direct relevance to the focus communities. 
Moreover, I have worked closely with VCC staff throughout to ensure that the questions being asked were relevant and valuable to Vanuatu as a whole.

However, two key concerns remain. First, I observe that I am limited in my ability to address all concerns regarding my gender and provenance. In particular regarding gender, although I worked with female research assistants for structured interviews, by necessity I did conduct semi-structured interviews with women. Given the power dynamics of gender in Vanuatu (cf. Bolton 2003), it is possible that female interviewees were intimidated or uncomfortable with these interviews. I remained aware of the dynamics involved and used three tactics to limit any discomfort: soliciting interviews well in advance, through my gatekeeper informant, and seeking completely voluntary participation; clear explanation of the informed consent procedure, which stressed that interviewees could terminate the interview when they wanted; and discussing potential problems with gatekeeper informants prior to interviews. Ensuring that other community members are present at all times has been suggested by some (Scheyvens and Storey 2003). In my experience, however, in the context of Malekula this may not always be appropriate, as internal power dynamics (such as between my gatekeeper informant and the interviewee) may serve to exacerbate the problem while being invisible to me (McCarter, unpublished field notes). In sum, I note that although I remained mindful of these issues, I cannot claim to have completely ameliorated the power dynamics inherent in the research relationship. Indeed, it seems likely that were this research to be undertaken by a ni-Vanuatu woman, the research design and findings would be different. Regarding gender, then, this thesis cannot (and does not) claim to give a balanced view.

Second, I note a general problem with institutionalised ethics agreements such as those of the HEC, which may not have the scope or the broad foundations of the ISE's Code of Ethics or the CRP. As noted by Smith (2005), ethics agreements can circumvent the collective nature of indigenous communities by focussing on individual-level rights and IPR, and can be biased by the need for ethics committees needs to advance research activities. Moreover, such 'top-down' approaches to ethical behaviour may not address some of the fundamental power dynamics outlined above (Smith 2005). As such, I attempted to design research which reflected the principles of the ISE's Code of Ethics and the foundations of the Cultural Research Policy. I reflect on my success (and otherwise) in this endeavour in the conclusion in Section 9.2.

\subsection{Data collection}

Data were collected between 2008 and 2010 on Malekula Island. I used both qualitative and quantitative methods, in accordance with the pragmatist approach that guided my pursuit of the research objectives (Table 3.1). The primary instruments for data collection were structured and 
semi-structured interviews, though I also used focus groups to pursue objective 3(b). Interviews used a variety of sampling techniques and were targeted at different participants, and interview schedules are included in Appendices 11.6 to 11.9. Full details of the data collection methods used throughout the thesis are included in the relevant chapters.

Table 3.1: Summary of research methods used in this thesis

\begin{tabular}{|c|c|c|c|c|c|}
\hline Method & Objective & Description & Timing & Sample & $\begin{array}{l}\text { Sampling } \\
\text { strategy }\end{array}$ \\
\hline \multirow{2}{*}{$\begin{array}{l}\text { Structured } \\
\text { interviews }\end{array}$} & $\begin{array}{l}\text { Scoping } \\
\text { study }\end{array}$ & $\begin{array}{l}\text { Structured interview conducted with } 120 \\
\text { participants regarding perceptions of TEK } \\
\text { change. Included trend analysis }\end{array}$ & 2008 & $\begin{array}{l}\text { Community } \\
\text { participants }\end{array}$ & $\begin{array}{l}\text { Snowball } \\
\text { sampling, } \\
\text { stratified by age } \\
\text { and gender }\end{array}$ \\
\hline & $\begin{array}{l}\text { One and } \\
\text { two }\end{array}$ & $\begin{array}{l}\text { Structured interview conducted with } 177 \\
\text { participants to elicit individual-level } \\
\text { ethnobiological knowledge and transmission } \\
\text { of key skills }\end{array}$ & $\begin{array}{c}2009- \\
2010\end{array}$ & $\begin{array}{l}\text { Community } \\
\text { participants }\end{array}$ & $\begin{array}{l}\text { Snowball } \\
\text { sampling, } \\
\text { stratified by age } \\
\text { and gender }\end{array}$ \\
\hline \multirow{3}{*}{$\begin{array}{l}\text { Semi- } \\
\text { structured } \\
\text { interviews }\end{array}$} & $\begin{array}{l}\text { One and } \\
\text { two } \\
\text { (piloting) }\end{array}$ & $\begin{array}{l}\text { Freelisting of medicinal plant domains for } \\
\text { objective two; creation of key skills list for } \\
\text { objective three; discussion of TEK } \\
\text { transmission and transmission changes }\end{array}$ & $\begin{array}{c}2009- \\
2010\end{array}$ & TEK experts & $\begin{array}{l}\text { Purposive, non- } \\
\text { random }\end{array}$ \\
\hline & $3(a)$ & $\begin{array}{l}\text { Semi-structured interviews regarding value } \\
\text { of TEK to formal school and vice versa }\end{array}$ & 2008 & $\begin{array}{l}\text { TEK experts, } \\
\text { teachers, } \\
\text { Officials }\end{array}$ & $\begin{array}{l}\text { Purposive, non- } \\
\text { random }\end{array}$ \\
\hline & $3(b)$ & $\begin{array}{l}\text { Semi-structured interviews regarding } \\
\text { triggers and barriers to formation of kastom } \\
\text { schools }\end{array}$ & $\begin{array}{l}2009- \\
2010\end{array}$ & $\begin{array}{l}\text { Kastom school } \\
\text { protagonists }\end{array}$ & $\begin{array}{l}\text { Purposive, non- } \\
\text { random }\end{array}$ \\
\hline Focus groups & $3(b)$ & $\begin{array}{l}\text { Group interviews with kastom school } \\
\text { committees }\end{array}$ & $2009-$ & $\begin{array}{l}\text { Kastom school } \\
\text { committees }\end{array}$ & $\begin{array}{l}\text { Purposive, non } \\
\text { random }\end{array}$ \\
\hline
\end{tabular}

\subsection{Study limitations}

In this chapter I have outlined the methodological approach I will use to address the objectives set out in Chapter One. This methodology allows me to address aspects of the variation, transmission, and maintenance of TEK and fill the literature gaps outlined in Chapter Two. As with any research this study has a number of inherent constraints and is limited in a number of respects. Four interlinked examples are noted below:

Rapid vs. in-depth approach: The methodological approach chosen is rapid rather than in-depth in nature. I spent two to three months in each community, and employed formal interview techniques in Bislama. This approach allowed me to compare variation between several communities and to rapidly build up a body of data examining the key areas of interest. It stands in contrast, however, to in-depth ethnographic methods, which typically seek to spend over a year in a community and base data collection on the incremental accumulation of knowledge over time (Bernard 1998). Moreover, such approaches would seek to use local vernacular languages during the interview process.

Ethnographic research approaches allow a deeper grounding of the research within the communities 
and an enhanced potential to describe and recognise nuance. They do not, however, offer the scope of the rapid methods, which build on earlier ethnobiological studies (as outlined in Section 2.4.1) and allow comparison, both between communities and with previous studies.

Reliance on the methodology of others: Following this, I note that the lack of ethnographic grounding in the focus communities somewhat constrains the methods and analysis I was capable of developing. In particular, it meant that I was largely reliant on the literature reviewed in Chapter Two to develop hypotheses for analysis, which may have limited the local applicability of the variables I chose to measure. For example, in Melanesia, clan membership is likely to be a critical variable in the structuring of knowledge within the communities, which means it would make an ideal variable for inclusion in the models described in Chapter Five (Sanga pers. comm., April 2012). However, because few studies of this type have been conducted in the region it was not included in the study methodology.

Publication-oriented thesis design: The construction of this work as a series of publicationoriented chapters, as opposed to a single dissertation, allows the effective targeting of literature gaps and the rapid dissemination of research results. It does, however, entail a trade-off of depth for breadth and a sacrificing of some local detail in favour of universally relevant, publication worthy, description. In point of fact, it is worth noting here that each of the core chapters (Five to Eight) could easily have been the subject of an entire thesis in their own right; and as such, do not claim to be exhaustive in nature. Rather, they are targeted directly at filling the literature gaps identified in Chapter Two.

Academic 'lens': More broadly, it is worth noting that this thesis is largely situated within the ethnobiological literature, reviewed in Chapter Two. This literature is the basis for my objectives and defines my approach to answering the key questions: as such, it represents the academic 'lens' through which I view my research. However, the broader questions with which this work is concerned could be (and have been) addressed from the vantage point of a number of different academic disciplines, such as sociology, anthropology, and political ecology. Each of these disciplines would have its own literature, theoretical perspective, and methodologies for defining and answering questions regarding TEK on Malekula. While I have tried to consider all the appropriate literature where possible, a full review of relevant work from every discipline would be a herculean task and would be quite beyond the scope of this thesis. 


\subsection{Chapter summary}

This chapter has outlined my approach to achieving the objectives set out above. I have described my own positioning with regard to the research process and the philosophical underpinnings of this research, which are important as they ultimately shape the nature of the research program. I then identified some key concerns regarding power dynamics and research, particularly those concerning academic research relationships with indigenous people and gender. I attempted to mitigate these power dynamics through the use of rigorous ethical procedures, which were outlined in Section 3.3.2. Finally, I sketched the methods used to gather data toward the key research objectives, and outlined four limitations of my approach to this topic. The next chapter outlines the setting of this research, Malekula Island in the Republic of Vanuatu. 


\section{Setting: Vanuatu and Malekula Island}

The preceding chapters have described the importance of TEK, as well as the methods and structure that I have used to address the objectives of this thesis. As noted in Section 2.1, TEK is fundamentally linked to place, and processes of erosion and persistence of TEK are contingent on a myriad of environmental, social, and historical factors. Therefore, the context in which my research was set is a critical aspect of understanding and contextualising the findings described in the core chapters.

In this section I detail the setting of this work in Vanuatu, including its ecological and cultural characteristics and a brief sketch of relevant historical developments. I also discuss the key concept of kastom and the characteristics of the formal school system in Vanuatu, as both are critical aspects of social and cultural change throughout the archipelago. I then describe Malekula Island and the focus communities, including details of the potential factors (such as presence of formal schools) that may influence the persistence, erosion, and maintenance of TEK.

\subsection{Geography, climate, flora, and fauna}

Vanuatu consists of over 80 islands scattered throughout the South-Western Pacific, between the latitudes of $13^{\circ}$ and $21^{\circ} \mathrm{S}$ (Figure 4.1). Some 67 of the islands are inhabited, from Aneityum in the south to the isolated Banks Islands in the north, but only 12 of the islands can be regarded as significant in terms of population (Bradacs 2008). Its closest neighbours are the Solomon Islands 170 $\mathrm{km}$ to the north, New Caledonia $230 \mathrm{~km}$ to the south, and Fiji $800 \mathrm{~km}$ to the east. The total land area is just over 12,000 $\mathrm{km}^{2}$, mostly made up of rugged and mountainous islands that range between 600 and 1200m in elevation (Mueller-Dombois and Fosberg 1998). Vanuatu is one of the most active regions of the Pacific ring of fire: there are small active volcanoes on four islands, and seismic events are commonplace throughout the group (Garanger 1996). 


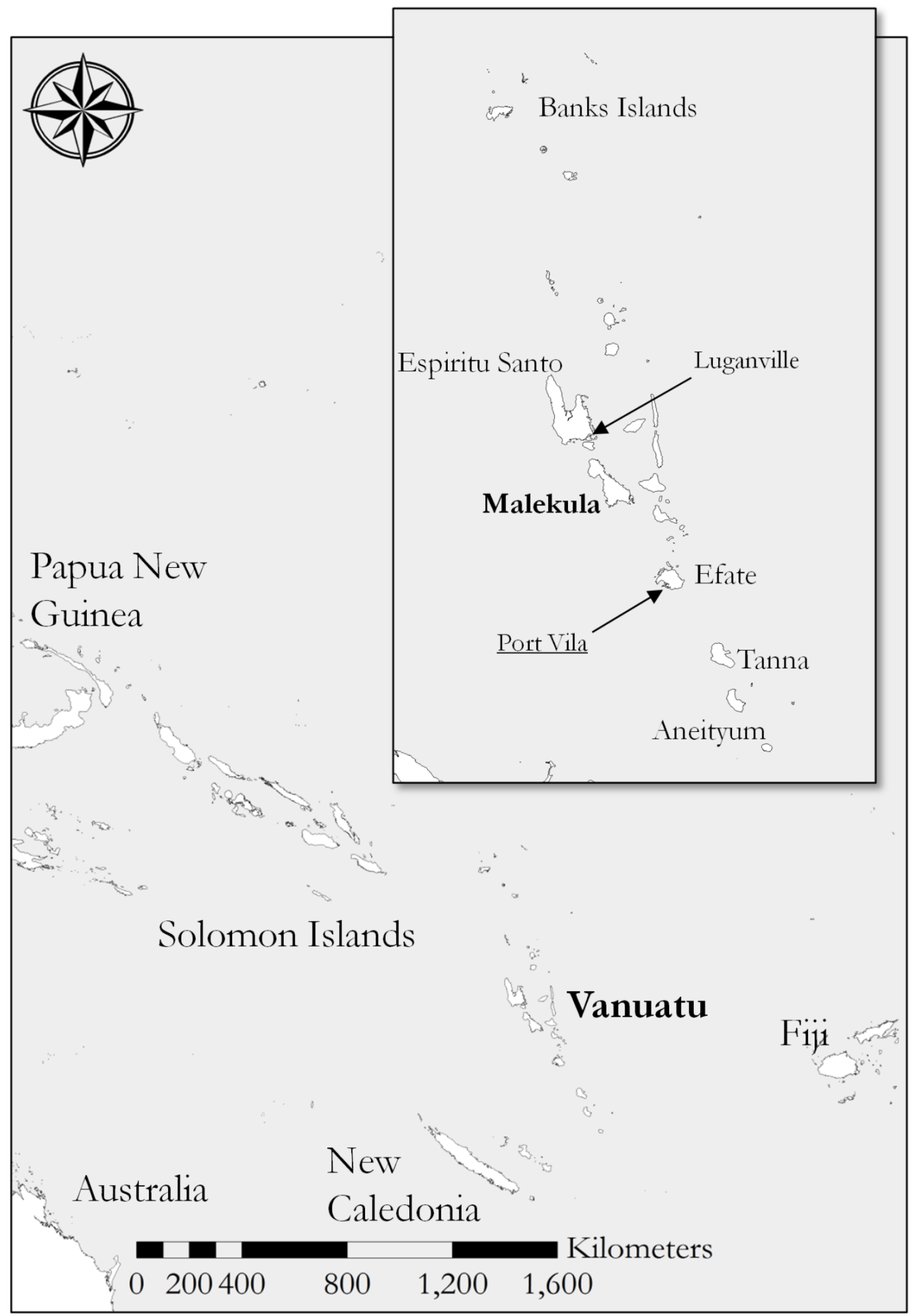

Figure 4.1: South Pacific and Vanuatu (inset) 
The climate of Vanuatu ranges between 'wet tropical' in the in the northern Banks and Torres islands (around $4000 \mathrm{~mm}$ of rain a year) to 'dry subtropical' in the southern section of the chain (around 2200 mm of rain per year) (Mueller-Dombois and Fosberg 1998). There are two distinct seasons: the cooler trade wind season occurs between May and October, during which the northern leeward sides of the islands experience mini dry spells; while the hurricane season lasts from November to April (Mueller-Dombois and Fosberg 1998). Median monthly temperatures range from $23.5^{\circ} \mathrm{C}$ in Aneityum to $26.1^{\circ} \mathrm{C}$ in the northern island of Vanua Lava (Mueller-Dombois and Fosberg 1998). During the hurricane season an average of four to six cyclones of hurricane strength can be expected in the Eastern Melanesian region, and in Vanuatu they have occurred an average of 2.6 times per year over the last 40 years (Chambers 1992). Vanuatu's climate is highly susceptible to the cycles of El Nino and La Nina, which, respectively, exacerbate the risks of droughts and flood. These climatic and geographic factors mean that Vanuatu faces a number of hazards, resulting in the Commonwealth Vulnerability Index ranking the country as the most vulnerable out of 111 developing countries (Warrick 2009).

Vanuatu is located in the biodiverse Indo-Malaysian region, and forms the easternmost bastion of the East Melanesian biodiversity hotspot (Conservation International 2009). The number of flowering plants has been estimated at between 900 (Chambers 1992) and 1200 (Cabalion et al. 1991), although as yet there has been no comprehensive description of the vegetation of Vanuatu as a whole (Mueller-Dombois and Fosberg 1998). Vanuatu is floristically less diverse than neighbouring groups, and has a much lower level of endemism than neighbouring groups such as New Caledonia (3,100 species in 24,000 $\left.\mathrm{km}^{2}\right)$ and Fiji (1,600 species in 18,000 km²) (Chambers 1992). The lack of endemism is likely to be a result of the relative geological youth of the group, which has left a short period of time during which the islands could be colonised. There remains, however, a considerable diversity in varieties of key crops cultivated and propagated by indigenous ni-Vanuatu, ${ }^{4}$ including breadfruit (Navarro et al. 2004), yam (Weightman 1989), and taro (Caillon et al. 2004).

Forested land covers about $75 \%$ of total land area, including exotic plantation forests (Rosillo-Cale and Woods 2003). Much of the natural forest is on steep and inaccessible land, which has limited its exposure to commercial logging activities (ibid.). Mueller-Dombois and Fosberg (1998) derive six primary vegetation types present throughout the archipelago, which vary according to altitude, substrate, windward vs. leeward orientation, and human influence. These include lowland rainforest, montane cloud forest, coastal vegetation, and secondary/cultivated woodland mosaics. The latter type is characterised by a predominance of fast-growing, heliophytic colonisers patterned with cultivated woodlands of commercial plantations of coconut (Cocos nucifera) and cacao (Theobroma cacao),

\footnotetext{
${ }^{4}$ Citizens of Vanuatu are referred to as ni-Vanuatu. The prefix $n i$ - derives from a preposition meaning 'of' in a number of languages throughout the country (after Lynch and Crowley 2001)
} 
and it is this vegetation type which surrounded each of the focus communities. A full vegetation map (though not one that necessarily corresponds with the classificatory system above) of Malekula Island is included in Appendix 11.14.

The fauna of Vanuatu is similarly species-poor, and most groups of animals have few representative species compared with the older, neighbouring islands of the Solomons, Fiji, and New Caledonia (Chambers 1992). Bats are the only naturally-occurring terrestrial mammal, though feral and domestic animals, three species of rats and the house mouse are also present. There are two (nonvenomous) snakes, and 19 species of lizard (mostly skinks and geckoes). There are also 121 species of birds (Bregulla 1992). Given the large areas of fringing reef habitat, it is no surprise to find that Vanuatu boasts a rich marine fauna: there are 295 species of stony coral, as well as 469 species of reef fish. Dugongs (Dugong dugon) and estuarine crocodiles (Crocdylus porosus) are also present in some areas, although the latter may be at risk of extinction (Chambers 1992).

\subsection{History}

The northern tip of the Solomon Islands was settled by around 28,000 years ago, at a time when low sea levels would have facilitated travel throughout the archipelago (Gorecki 1996). However evidence of settlement is not noted in Vanuatu until the expansion of the Lapita people, who rapidly spread over 4,500 $\mathrm{km}$ in one of the great migrations in human history (Bedford and Galipaud 2011). Archaeological evidence appears to date human settlement as back as far as 3,200 years B.P (Bedford and Galipaud 2011), a timeframe that is roughly corroborated by recent linguistic evidence (Gray et al. 2009).

Around 2,500 years ago, a second wave of migrants arrived from the Solomons, bringing with them stone tools and another style of pottery (the Mangaasi style). By around 2000 years ago the Lapita style of pottery becomes less frequent in the archaeological record, and knowledge of the inhabitants of this era becomes less detailed (Spriggs 1996). There is some evidence, however, that there was influence from Polynesia to the East, inferred by the borrowing of material culture and of social organisational structures (such as the presence of chiefs on some islands) (MacClancy 2002).

The first European contact with Vanuatu was through the Spanish explorer de Quiros, who anchored off Big Bay (Espiritu Santo) in May 1606. This did not result in permanent settlement, and until 1768 the islands remained out of reach of the European explorers. However, during the latter half of the $18^{\text {th }}$ Century, contact was made by de Bougainville (French) and Cook (British), ushering in a period of rapid cultural change and decimation. Driven by trade (especially for the valuable sandalwood) and missionary zeal, the population of foreigners in Vanuatu expanded rapidly, and 
Vanuatu (or the New Hebrides, as it was known until independence in 1980) soon became famous in Europe as a place of "...fever-ridden islands and inhabited by unfriendly cannibals" (MacClancy 2002: $\mathrm{p} 40$ ). For much of the 19 ${ }^{\text {th }}$ century, British subjects from Australia formed the bulk of the foreign population. However, the establishment of the Caledonian Company in 1882 led to a surge in the number of French nationals in the islands, and they soon outnumbered the British two to one (U.S. Department of State 2011).

As across much of the Pacific, the missionary contact was to form the most pervasive interaction of ni-Vanuatu with foreigners. After a faltering start, Anglican, Presbyterian, and Catholic forms of Christianity spread throughout the islands, and by the early $20^{\text {th }}$ century there were at least nine mission stations and an increasing proportion of local adherents (MacClancy 2002). The process of conversion was complex on most of the islands: typically, missionaries would set up stations on the coast, and through a combination of 'push' (such as local competition) and 'pull' factors (such as the availability of western medical care) local interior populations would gradually coalesce on the coast (Lake 1985). As a result, Vanuatu is now a predominantly a Christian country, though this worldview is overlaid and influenced by indigenous spirituality to varying degrees in different places (Hickey 2006b). Regenvanu (2005) argues that the process of conversion was assisted by 'psychological warfare' on the part of the missionaries, who imparted a message of the inferiority of indigenous traditions, and would use strategies designed to forcibly convert locals (such as the withholding of medicines for the sick).

Trading activities were the other major source of contact between ni-Vanuatu and foreigners. In addition to the sandalwood trade (which had largely ceased by 1860) settlers in Vanuatu focussed on growing cotton, coffee, cocoa, bananas, and coconuts (MacClancy 2002). They often employed large numbers of ni-Vanuatu to work on the plantations, resulting in significant migration within and between islands. The international trade in labour between Melanesia and the sugarcane fields of Northern Queensland and Fiji (known as 'blackbirding') also had a significant impact on ni-Vanuatu populations. Indeed, between 1860 and 1900 some 40,000 ni-Vanuatu were moved overseas, of which only 30,000 ever returned back to their communities (MacClancy 2002). Whilst the blackbirding trade over this period is now characterised by stories of injustice and poor working conditions, for some ni-Vanuatu it was also an exciting opportunity to gain wealth and experience a new way of life (MacClancy 2002). This period was also critical in the formation of Bislama, a neo- 
Melanesian pidgin which has become the lingua franca of the archipelago, as the vast linguistic diversity of Vanuatu was forced to interact for the first time (Crowley 1990). ${ }^{5}$

For ni-Vanuatu, however, the primary dynamic of $19^{\text {th }}$ and early $20^{\text {th }}$ centuries was one of depopulation (Regenvanu 2005). During this period, it is estimated that over $80 \%$ of the population was killed by disease (especially pneumonia, dysentery, influenza, measles, diphtheria, and whooping cough) and through the introduction of firearms, which greatly exacerbated the human cost of skirmishes. There remains a paucity of reliable data: the best examples come from islands such as Aneityum, where a 95\% drop in population was recorded between 1850 and 1905 (Spriggs 1985), and Erromango, where a population of 4,500 before the discovery of sandalwood was reduced to only 500 by 1930 (MacClancy 2002).

In 1906 the French and British colonial governments, recognising mutual interest in the natural and human resources of Vanuatu, agreed to administer the islands and formed a joint government known as the 'Condominium'. This was a fairly unique arrangement, with both governments maintaining independent education, judicial, and governance systems. Throughout this period neither government took much of an interest in the indigenous inhabitants of the islands, and the inefficiency of governance quickly became legendary (Larcom 1990). Ni-Vanuatu nationalism grew slowly throughout middle part of the $20^{\text {th }}$ century, aided by the arrival (and mythologising through cargo cults) of American troops during World War Two. In the early 1970's the first political party (the New Hebrides National Party, later the Vanua'aki Party) was formed and after a period of intense unrest elections were held in 1979. Independence was declared on the $30^{\text {th }}$ of July 1980 , and the new republic of Vanuatu (meaning 'our land') under new Prime Minister Father Walter Lini was ushered into the forum of new Pacific nations. From the beginning, the new government emphasised the importance of indigenous ni-Vanuatu kastom and culture as exemplifying the 'Melanesian way' (Tonkinson 1982). At a governmental level, the 30 years since independence have been characterised by political schisms and splits.

\subsection{Current snapshot}

In 2009 the population of Vanuatu was 234,023 (Vanuatu National Statistics Office 2009). 94\% of the population are indigenous ni-Vanuatu, with the remainder originating from a number of countries including Europeans, Vietnamese, Chinese, and other Pacific nations (Vanuatu National Statistics Office 2006). The population is extremely youthful, with a median age of 20.5 and nearly $60 \%$ under the age of 24 (Vanuatu National Statistics Office 2009). 80\% of ni-Vanuatu are based in rural

${ }^{5}$ Of course, the various language groups of Vanuatu had been interacting on a limited scale (i.e. with their close neighbours) for millennia (Tryon 1996). However, the scale of the mixing during the blackbirding era was such that new means of communication were required, which was a key driver of the formation of Bislama (Crowley 1990) 
areas, although the urban population of 57,195 is growing rapidly at a rate of $3.5 \%$ per year (Vanuatu National Statistics Office 2009). ${ }^{6}$ The two major urban centres are the capital Port Vila (Efate) and Luganville (Santo).

Agriculture is important in Vanuatu and 98\% of the rural population is engaged to some degree, usually utilising shifting cultivation systems based around the common staples of taro and yam (Vanuatu National Statistics Office 2006). The economy is heavily reliant on agricultural exports: copra (coconut meat) accounts for 34-56\% of gross national exports, followed by markets for timber, beef, cacao, and kava (Piper methysticum) (Vanuatu National Statistics Office 2006). In total, these crops make up 75\% of total agricultural exports and 20\% of GDP (Rosillo-Cale and Woods 2003). Other key components of the economy include tourism, spurred on by interest from several large cruise ship companies, and the financial sector, which benefits from the tax-haven status of Vanuatu.

Culturally, the most outstanding feature of Vanuatu is its diversity. In keeping with the biocultural diversity that characterises the Indo-Melanesian region, an estimated 105 (Tryon 1979) or 106 (Lynch and Crowley 2001) languages are found on the islands throughout the country. ${ }^{7}$ This may represent the highest diversity of languages per capita of any country in the world; indeed, although there are relatively few speakers, the languages of Vanuatu constitute fully $10 \%$ of the languages of its parent family Austronesian (Tryon 1996). There are major language divisions between northern, central, and southern sections, which are supported by a number of commonalities in material culture and dialects that appear to have been linked through 'dialect chaining' (Tryon 1996). These dialect chains were connected with extensive trade networks which linked every island in the group, based on items including pigs, wives, ochre, pottery, mats, and songs (Bonnemaison 1996; Tryon 1996).

The varied cultural traditions of Vanuatu remain a central facet of everyday life. Indeed, Vanuatu is one of the places in the Pacific, and the world, where cultural traditions have been retained to the greatest extent (Regenvanu 2005). A large proportion of ni-Vanuatu live on customary land, held and used according to customary tenure; satisfy most of their food and nutritional needs through subsistence farming; live in communities where the governance structure is largely derived from customary forms and principles; speak their vernacular language; and have been involved, at some point, in customary ceremonies at important life stages, such as weddings or funerals (Regenvanu 2005). A central plank of the importance of tradition in Vanuatu is the land tenure system, which (in theory) is guaranteed to the custom owner by the Constitution of Vanuatu, thus ceding most

\footnotetext{
${ }^{6}$ This is high in global terms: by way of comparison, the global urban growth rate is 1.97\% (United Nations 2009).

7 The delineation of languages can be a political, rather than a purely linguistic, task: as noted by Muhlhausler (1995): "...the identification of languages and their subsequent naming is far from being an act of objective description, and it can constitute a very serious trespass on the linguistic ecology of an area...” (p 5)
} 
responsibility for the management of land to the local level (Haccius 2011). ${ }^{8}$ Therefore, for most of the population of Vanuatu, life is organised around customary social institutions and ancestral land.

\subsubsection{TEK in Vanuatu}

Accordingly, TEK holds a central importance in contemporary ni-Vanuatu society. The strong bonds and institutions that have an important part of traditional systems are manifested as stocks of human and social capital (Hickey 2006b). At least three specific examples of TEK in Vanuatu have been noted in the literature:

Natural resource management: Given the land tenure arrangements noted above, the most visible application of tradition to the environment is in the form of natural resource management, particularly of near shore marine resources (e.g. Hickey 2003a; Hickey 2003b; Hickey 2005; Hickey 2006a; Hickey 2006b; Hickey and Ruddle 2008). There is significant variation in practices between islands throughout the group, and numerous customary rules and protocols permeated customary life and served to control access to resources. This made the direct conservation of resources possible throughout Vanuatu (Hickey 2003a). Specific measures included spatial-temporal refugia, privileged user rights, closed seasons, and gear restrictions. Detailed management schemes remain in place in many areas, and there have been examples of successful contemporary conservation areas based on both western and customary techniques (Hickey 2006a). Some studies have shown, however, that customary resource management in Vanuatu does not relate to underlying distributions of fisheries stocks, and may have issues with equity (Mees and Anderson 1999); or that the institutional arrangement in Vanuatu is insufficient for integrated fisheries management (Lane 2006).

Natural hazard management: TEK would have been vital to mitigating the impact of the regular natural hazards which occurred on the islands throughout Vanuatu. In particular, extreme weather events in the shape of hurricanes regularly cause immense damage to food production systems, houses, and infrastructure, particularly in the northern Banks and Torres Islands. A number of practices were important, including the maintenance of a diverse range of crops in addition to using wild or feral species (see also Caillon et al. 2004; Navarro et al. 2004; Weightman 1989); fragmentation and regular rotation of garden land (Weightman 1989); regular inter- and intracommunity cooperation through the promotion of feasting and trade (Bonnemaison 1996); and the features of some buildings throughout the archipelago (Campbell 1990; Campbell 2006). TEK

\footnotetext{
${ }^{8}$ In practice, Vanuatu maintains two separate tenure systems: a formal system inherited from the colonial period, and an un-codified customary system. The latter system retains a prominent importance, as social organisation is closely bound to land rights and management (to the extent that place of origin and personal identity have been described as synonymous; Haccius 2011). Currently, the “...interaction of the customary and formal land tenure systems often has negative consequences, such as dispossession of customary land owners and deterrence of investors. Neither system properly understands the other..." (Haccius 2011: p 2).
} 
systems may also have been useful in predicting, and thus facilitating preparation for, extreme weather events (Campbell 2006). Other work has utilised TEK systems on Ambae to improve volcano monitoring systems (Cronin et al. 2004).

Traditional medicine: Vanuatu's cultural diversity is reflected in a large and varied body of traditional ethnomedical knowledge (Bradacs et al. 2011). Customary medicines are an integral part of everyday life for most rural inhabitants, especially given the difficulty of access to western medical care in many areas (Grace 2001). As noted, a complete description of Vanuatu's flora are lacking, though there have been a limited number of ethnomedical investigations (Billo et al. 2005; Bourdy et al. 1996; Bourdy and Walter 1992; Bradacs 2008; Bradacs et al. 2011; Cabalion et al. 1991; Weightman 1989). These studies have reflected a relatively high richness of medicinal plants species, compared to other quantitative ethnomedical studies such as those reviewed in Begossi et al. 2002. For example, Bradacs et al. (2011) documented 133 medicinal species across three islands, while Bourdy and Walter (1992) recorded around 120 species used by women in health and reproduction. Such studies confirm anecdotal accounts that traditional medicine is an important source of resilience. There are serious concerns surrounding the protection of the IPR of medicinal TEK holders in Vanuatu, driven by cases of bioprospecting in other countries (Regenvanu 2005), and the patenting of a kava extract (kavalectone) by US and German companies (Lamont 2009; see Section 2.3.3).

It is worth noting here that issues of ownership and visibility of TEK in Vanuatu are mutable depending on the type of knowledge in question. Much TEK is mundane (i.e. in daily usage) and is circulated widely at national, local, and clan levels. For example, some ethnomedical knowledge (such as the use of particular plants to cure headaches) may be found throughout the archipelago, and may have no particular spiritual or secret significance. Other types of knowledge, however, are closely held within 'knowledge communities', which are defined by a collective responsibility that necessitates the guardianship of secret and sacred information (Sanga, pers. comm.; May 2012). The transmission of this sacred knowledge is closely guarded and is dependent on the background and rank of the learner. These 'knowledge communities' exist at clan or sub-clan levels, and may be closely linked with animistic beliefs and the strict systems of graded rank that were prevalent across much of Vanuatu before Christianity (Bonnemaison 1996). Although strict adherence to the graded rank system is now no longer the norm in Vanuatu, ideals of rank and ownership still wield considerable influence on social structure in the contemporary Malekulan context (Curtis 2002). I'd note at this stage that the nature of my methodology and approach to the research questions means that this thesis only deals with mundane knowledge, or TEK that is widely shared throughout the community. 


\subsection{Kastom}

Kastom is one of the central themes of contemporary life in Vanuatu, and it is difficult to discuss TEK in Vanuatu without first briefly exploring the concept. In particular, it and forms a key part of discussions in Chapters Seven and Eight. According to Terry Crowley's (2003) Bislama dictionary, kastom refers to 'custom', 'tradition', or 'traditional'. Similarly, Bolton (1999a) notes that for local fieldworkers (discussed in Section 0), kastom is understood to be anything derived from pre-colonial knowledge and practice ( $\mathrm{p} 7)$. However, the definition of kastom has proven to be a difficult task, as it is being constantly re-interpreted over time, means different things to different actors, and is embodied at a number of scales ranging from the local to the national (Regenvanu 2005). At a broad scale, kastom is simply lived experience, and is central to everyday life:

“...kastom...is not merely an odd collection of dances and rituals, but a whole way of life that dictates almost all of one's actions, and provides its own particular interpretation for almost anything that happens. Distinctions used to describe modern Western society may not necessarily be applicable to it. 'Social organisation', 'economics', 'politics', 'communications'....may be separate categories in the accounts of the French (or the English) way of life, but in kastom they are woven together into such an intricate complex whole that our understanding...would be gravely distorted if they were to be used here..." (MacClancy 2002: p 21).

In addition, Bolton (2003) stresses that kastom is defined by the people who enact it as something belonging to them, in opposition to that which comes from outside:

“'Kastom” is a cognate terms for culture in Bislama, Vanuatu's lingua franca. It is used to refer to knowledge and practice that ni-Vanuatu understand to be authentically their own, deriving from their pre-colonial past and from their place...it is a term that derives from contact with outsiders yet describes what belongs to people of the place...” (p 6).

There have been a diverse range of academic discussion around the nature of kastom over the past three decades (see Bolton 2003 p 23 for a review), however the thread that underlies most of them is an idea of kastom as a multi-faceted and contested concept, that is " ...an apt and powerful symbol precisely because it can mean (almost) all things to all people" (Keesing 1982, cited in Bolton 2003: $\mathrm{p}$ 24).

The term was first used extensively by the nascent independence-era political movement during the 1970’s (Tonkinson 1982). As noted by Bolton (1999b), “...the nation’s incipient politicians made much of kastom as representing a unified identify for all the indigenous inhabitants of the archipelago. At this stage, a national discourse about the importance of kastom began to develop" ( $\mathrm{p}$ 349). This 
process was assisted by three key events: the development of a regular radio slot, run by the French broadcaster Paul Gardissat, which focussed on the importance of 'kastom, kalja, mo tredisen' (custom, culture, and tradition); the first National Arts Festival in 1979, strongly promoted by the Vanua'aki Party; and the development of the Vanuatu Cultural Centre (VCC) under the curatorship of British anthropologist Kirk Huffman (Bolton 1999b; Bolton 2003). As such, this period saw the development of kastom as a symbol of resistance against colonial rule by ni-Vanuatu, and the postindependence government used 'kastom' and the Melanesian way as the principle guide for the development of a raft of stridently anti-colonial policies during the 1980's (Regenvanu 2005).

However, 'kastom' has always embodied a number of tensions. In this initial period, perhaps the strongest tension was between national, regional, and local versions of kastom. While the Vanua'aki Party and the VCC were espousing one version of kastom from the capital Port Vila, the concept was also being used to claim legitimacy for separatist movements, such as the Nagriamel movement (see MacClancy (2002) or Vigestad (1984) for more information). Tonkinson (1982) writes that community members were confused as to whether a return to kastom values would entail a return to a life devoid of the trappings of modernity, such as outboard motors. Moreover, there was concern about exactly whose kastom would be represented at a national level. This lack of definition was valuable to nationalist politicians for a time, because "... as long as the notion of kastom remained vague and undefined, or at least undifferentiated, it was an excellent vehicle for the expression of the indigenous people's sense of cultural loss and anger at Europeans... but Vanuatu's very considerable cultural diversity meant that shared kastom was inadequate as a basis for the creation of a countrywide, well organised, popular political movement" (Tonkinson 1982: p 308).

More recently, the use of the kastom in the national arena has caused tensions between groups of niVanuatu, as it is often used to claim legitimacy or historical precedent to the actions of certain parties. This 'invention of tradition' has been widely discussed in the Pacific literature, and illustrates that 'kastom' and 'tradition' do not necessarily represent historical roots (Bolton 2003). For example, the National Council of Chiefs (NCC) have agitated for a greater official role in the state, on the basis that they are the true kastom leaders of Vanuatu. The legitimacy of this interpretation has been questioned by some, as for most of Vanuatu the chiefly system was only enacted during the colonial administration (Regenvanu 2005). The 'kastom' role of women has also been debated. While bodies such as the NCC have promoted a version of kastom which holds that a woman's role should be one of 'quiet subservience', this is not strictly accurate for most of Vanuatu (Regenvanu 2005). Indeed, this version of kastom, in which the role of women is immobilised in time and space, depends on an interpretation of gender relations that exaggerates male dominance (Jolly 1994), and may draw more from the views and practices introduced by the missionaries (Regenvanu 2005). The recognition that women's kastom is legitimate, and that women have the right to operate and be visible in Vanuatu, is 
a relatively recent one that stems from the first meeting of women fieldworkers in 1991 (Bolton 2003).

Finally, the development of the national discourse on kastom has also served to define local and regional differences, and so has served to link particular versions of kastom to place (Bolton 1999c). As noted by Joan Larcom (1990), working in South Malekula, the idea that the local Mewun people had kastom that was intrinsically theirs had changed their ideas about knowledge and practice: "...the Mewun [a local group] have not perceived themselves as a separate cultural entity until recently...growing Mewun consciousness of the power inherent in cultural distinctions has arisen with their awareness of national politics and kastom" ( $\mathrm{p}$ 188-199). Indeed, the concept of kastom as a whole has developed in response to other ways of thinking (specifically, of the colonial administrations), and so serves to identify a version of 'ni-Vanuatu' that is unique from the 'other'. This has been evident at national, regional, and local scales. In this sense, kastom refers to dynamic reconceptualisations of the ways in which the past is represented in the present and future, such that "...kastom is not so much a nostalgic recuperation of the past, nor even a conservative statement about the power of 'tradition' in the present, but a projection of individual and collective power into the future" (Curtis 2002: p 241).

Although 'kastom' is essentially fluid in nature, much of the discourse surrounding traditional knowledge and practice in Vanuatu is driven by concerns that they are at risk in contemporary social contexts. Commentators have speculated that the same drivers of TEK loss observed in other parts of the world (in particular formal education) may also be at work in Vanuatu (Regenvanu 2005; see Section 4.6). Other concerns are driven by a lack of governmental recognition and protection of TEK, and a national 'psychology of dependency' that stems from the practices of the early missionaries and results in "...the view that our own custom is somehow inherently flawed and is not as good as what comes from outside" (Regenvanu 2005: p 4). A third perceived threat to kastom is land alienation throughout the archipelago. Although the constitution of Vanuatu is intended to ensure that land remains with the customary owner, land can be leased for up to 75 years and must be effectively re-purchased by the owner at the end of the lease (Haccius 2011). Legislative inadequacies such as this are compounded by the difficulties in linking concepts of individual ownership with Melanesian norms; a lack of information and understanding on the part of the custom owners; and a lack of governmental control and oversight (Haccius 2011; Regenvanu 2008). Given the inseparable links of kastom and TEK with land, the alienation of land represents a direct threat to traditional knowledge and practice (Regenvanu 2008). 


\subsection{The Vanuatu Cultural Centre and the fieldworkers}

The Vanuatu Cultural Centre (VCC) is the lead agency in Vanuatu for the protection and maintenance of ni-Vanuatu kastom and culture. It dates to 1956, and has grown in importance since the appointment of Kirk Huffman as its first salaried curator in 1977. The VCC is a statutory body under the laws of Vanuatu, and is governed through the National Cultural Council of Vanuatu. It serves as an umbrella organisation that includes, most notably, the National Museum of Vanuatu, and is located in the capital Port Vila. There is a regional satellite of the VCC on Malekula Island. Known as the Malekula Cultural Centre (MCC), it was opened in 1991, and seeks to provide support for kastom and culture around the island (McCarter, unpublished field notes).

As noted above, the VCC played a key role in discussions of kastom during the early days of independence. In keeping with the newly independent spirit of the Republic, the VCC declared a moratorium on all humanities and field-based research in 1985 that lasted for nine years (Bolton 1999a). During this time, with very few exceptions, no new research projects were carried out, and cultural research by foreign academics only recommenced after the enactment of the Cultural Research Policy (Regenvanu 1999). The lifting of the moratorium was spurred by the increasingly urgent mandate of the VCC to protect and develop kastom throughout the country, a task that was beyond the capacity of the resident staff (Regenvanu 1999).

There remained, however, significant research activity within Vanuatu, mostly under the auspices of the fieldworker program (Tryon 1999). This program was developed through the 1980's and 1990's under the leadership of Huffman and the linguist Darrel Tryon, and consisted of a growing group of volunteer community members who aimed to record and maintain kastom, and particularly vernacular language, within their own communities. The enactment of the VCC's Women's Culture Program in 1991 led to the formation of a network of women's fieldworkers, and both groups have met every year since that point (Bolton 1999a). ${ }^{9}$ The role of the fieldworkers has developed and matured over the years, both through the influence of the workshop facilitators (in particular Huffman, Tyron, and Bolton), and as a consequence of interaction amongst themselves. Currently, while much of the work of the fieldworkers is in documentation and recording, they also run a variety of projects from the reopening of traditional dancing grounds to the revival of old ceremonies (Bolton 1999b). As noted above, they are also critical figures in terms of research in Vanuatu, and most foreign research is directed and guided by the advice and connections of the fieldworkers. Importantly, it is the fieldworkers who have taken the responsibility of conserving kastom within their own communities:

\footnotetext{
${ }^{9}$ Unconfirmed reports from Vanuatu indicate that there may be no workshop in 2012, for the first time in 30 years.
} 
and in doing so, they are key figures in constructing the discourse around kastom and tradition at the local level (Regenvanu 1999).

The VCC maintains a number of other ongoing projects aimed at the revitalisation of kastom in Vanuatu. These include:

1. Promotion of the 'kastom economy', during which they placed a heavy influence on the importance of traditional wealth items (such as mats, traditional food items, and pigs) as a means of ensuring self-reliance and the maintenance of social capital (Regenvanu 2007a; Regenvanu 2007b). This included innovative projects such as 'pig banks' on Malekula (Plate 4.1), which aimed to increase the importance and availability of traditional forms of wealth, while raising awareness about the opportunities afforded by Vanuatu's unique cultural heritage (Regenvanu 2007a).

2. The development of educational resource books for use in schools which emphasise the history and emergence of Vanuatu as a nation (Lightner and Naupa 2005). Until these books were produced schools in Vanuatu had little focus on national matters, and instead taught material derived from New Zealand and Australian curricula.

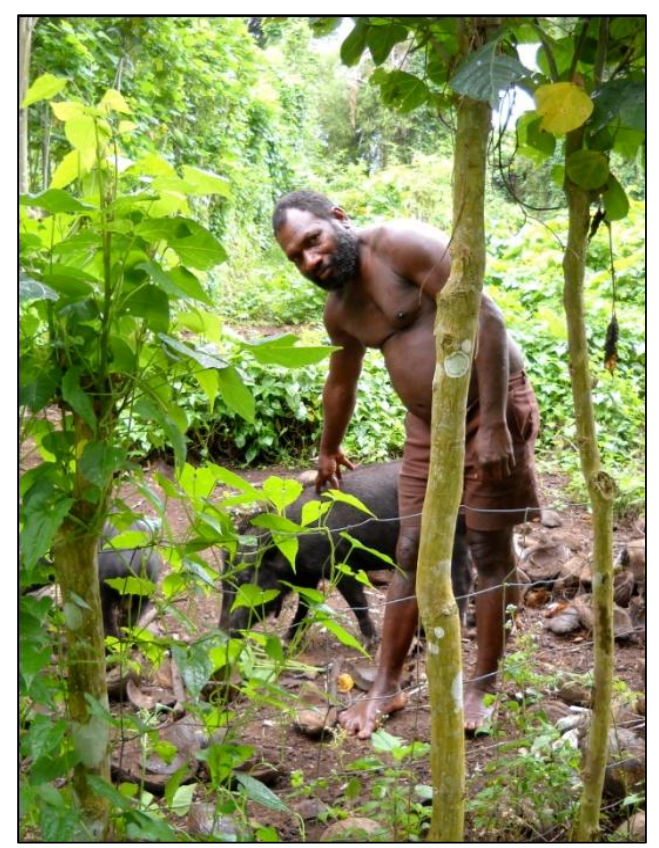

Plate 4.1: James Teslo, a fieldworker from interior South West Bay, with pigs in his 'pig bank'. Photo by author, used with permission of subject

3. The Vanuatu Young People's Project, which was initiated in 1997 to research the role that kastom and culture played in young people's lives in Port Vila (Regenvanu 2005). Currently, the project runs a number of youth 'kastom schools', both in order to advocate and facilitate the uptake of vernacular language and traditional knowledge in Port Vila, and to give a forum in which young people's voices can be heard (Vanuatu Cultural Centre 2005).

4. The development of curriculum modules for use in schools in order to facilitate the teaching and transmission of TEK (Baeraleo 2010; Regenvanu 2002; Regenvanu 2004). This project emerged from collaboration between the Local and Indigenous Knowledge Systems (LINKS) group of UNESCO and the VCC, and seeks to increase contact between the schools and resource people and experts within the community. This project was aimed at addressing some of the key failings of the formal education system, as discussed below. These units 
were produced by Sue Baraeleo and the VCC, and were scheduled for distribution during 2011. No information is available at the present time as to their success or otherwise.

\subsection{Formal education in Vanuatu}

Amongst the myriad drivers of cultural change present in Vanuatu, the formal education system has been singled out for criticism by a number of authors (Baeraleo 2004; Regenvanu 2002; Sanga et al. 2004). Formal education in Vanuatu was initially the preserve of French and British missionaries, and until the 1960's neither colonial administration took any interest in the education of ni-Vanuatu (Lake 1985; Siegal 1996). Both administrations slowly took control throughout the 1970's, until responsibility was combined under the new Vanuatu Ministry of Education after independence in 1980. For much of the 1980's and 1990's education priorities were driven by those of the aid agencies who provided much of the funding, and curricula continued to be based on the English and French models of the colonial administration. Currently, the formal school system is split into primary (years 1-6) and secondary (years 7-12) phases. At primary level, most schools are local and village-based. Secondary schools are less common, and pupils usually travel and board at the schools.

At present, $85.9 \%$ of pupils between the ages of 6 and 13 are enrolled at school, which has translated into a literacy rate of $84.8 \%$ for those above 15, 64\% of children with basic English abilities, and 37\% of children with basic French abilities (Vanuatu National Statistics Office 2009). Although those figures, in the context of the wider Pacific, are impressive, a number of recent reviews have criticised the current orientation of the formal school system throughout the country. For example, the Rethinking Vanuatu Education Together program, which brought together over 200 participants from a variety of stakeholder groups, concluded that "...three decades of considerable investments in education has not made a significant impact on ...educational development" (Sanga and Niroa 2004: p13), and a variety of contributions stressed the need for considerable reform to allow the input of indigenised and localised content (e.g. Baeraleo 2004; Nako 2004; Niroa 2004; Nirua 2004; Regenvanu 2004).

Specifically, scholars have argued that the current system fails on a number of fronts (see Regenvanu 2005 for more information). First, it removes children from the traditional learning context at an early age: at primary school, children are expected to attend for at least 30 hours per week, and at secondary level they commonly board at the schools. Second, it does not cater for the entire population, and most pupils are 'pushed out' by year 10 due to a lack of resources. Third, in maintaining a centralised curriculum based on New Zealand and Australian models and subjects (such as Science, English/French, and Maths), it ignores and devalues the diversity of indigenous knowledge and practice throughout the country. Fourth, it provides no instruction in values of issues 
related to citizenship or identity. Fifth, schools usually employ non-local teachers and are not integrated with communities and traditional power structures As a result, it has been argued that the school environment does not adequately prepare students for life in the urban environments of Port Vila and Luganville, but moreover that it prevents them from leading fulfilling and active roles within the village environment (Niroa 2004).

The inclusion of vernacular languages into education in Vanuatu has been a site of considerable debate (Siegal 1996). In the early days of formal education, missionaries often taught in vernacular languages, in order to more effectively educate and proselytise. However the focus in recent decades has been on education in English or French, despite the benefits of mother-tongue education being recognised by the international literature (Siegal 1996). Recently, there have been a number of initiatives, especially under the Education Master Plan (2000-2010) aimed at initiating the use of vernacular language in a series of pilot schools. These programs failed, however, due to a lack of capacity on the ground (Nako 2004). One key barrier to the success of vernacular education is the inherent difficulty in facilitating the teaching of vernacular language with such a diverse population and limited resources: “... any program of vernacular instruction in Vanuatu schools must be implemented with the greatest care and preparation. A massive job of linguistic surveying, linguistic description and development or improvement of writing systems therefore lies ahead before all of the vernaculars of the country can even be thought of being brought into the program for initial vernacular education..." (Crowley 2000: p 2). This is especially so because, ideally, a program of vernacular education will reflect the values and knowledge of the community in which it is based (Paviour-Smith 2005; Shipman 2008).

There are signs that steps are being taken in Vanuatu toward the inclusion of more local content in schools. A recent review of the curriculum statement acknowledges that "...the current curriculum is not designed for Vanuatu's diverse cultures and communities. Despite significant budget commitments to education by governments and other external agencies, our national curriculum is in need of reform so it reflects our national identity" (Vanuatu Ministry of Education 2010: p 2), and goes on to propose significant reform around the model proposed by Delors (1996): see Section 2.6.2 above). Moreover, there have been recent reports from Vanuatu that vernacular education is to be taught in all primary schools to year three, though I have yet to locate any published sources confirming this (M. Durand, pers comm. October 2011). 


\subsection{Malekula Island}

The primary location for this research was Malekula Island, ${ }^{10}$ in the central Malampa province (Figure 4.2). Geographically, Malekula is typical of many islands in Vanuatu, with steep-sided mountains rising to between 600 and 800 meters in the interior and southern sections, a narrow coastal plain along the eastern and northern coastlines, and several small islands off the coasts surrounded by fringing reefs. Of the 206,756 ha of Malekula, over 75,000 ha is forested, with much of the remainder on the coastal plains now dominated by large commercial plantations of coconut and cacao (Tacconi and Bennet 1993; Vanuatu National Statistics Office 2006). A map of dominant vegetation types is included in Appendix 11.14.

${ }^{10}$ In other work Malekula is sometimes spelled Malakula or, in the French, Malicollo 


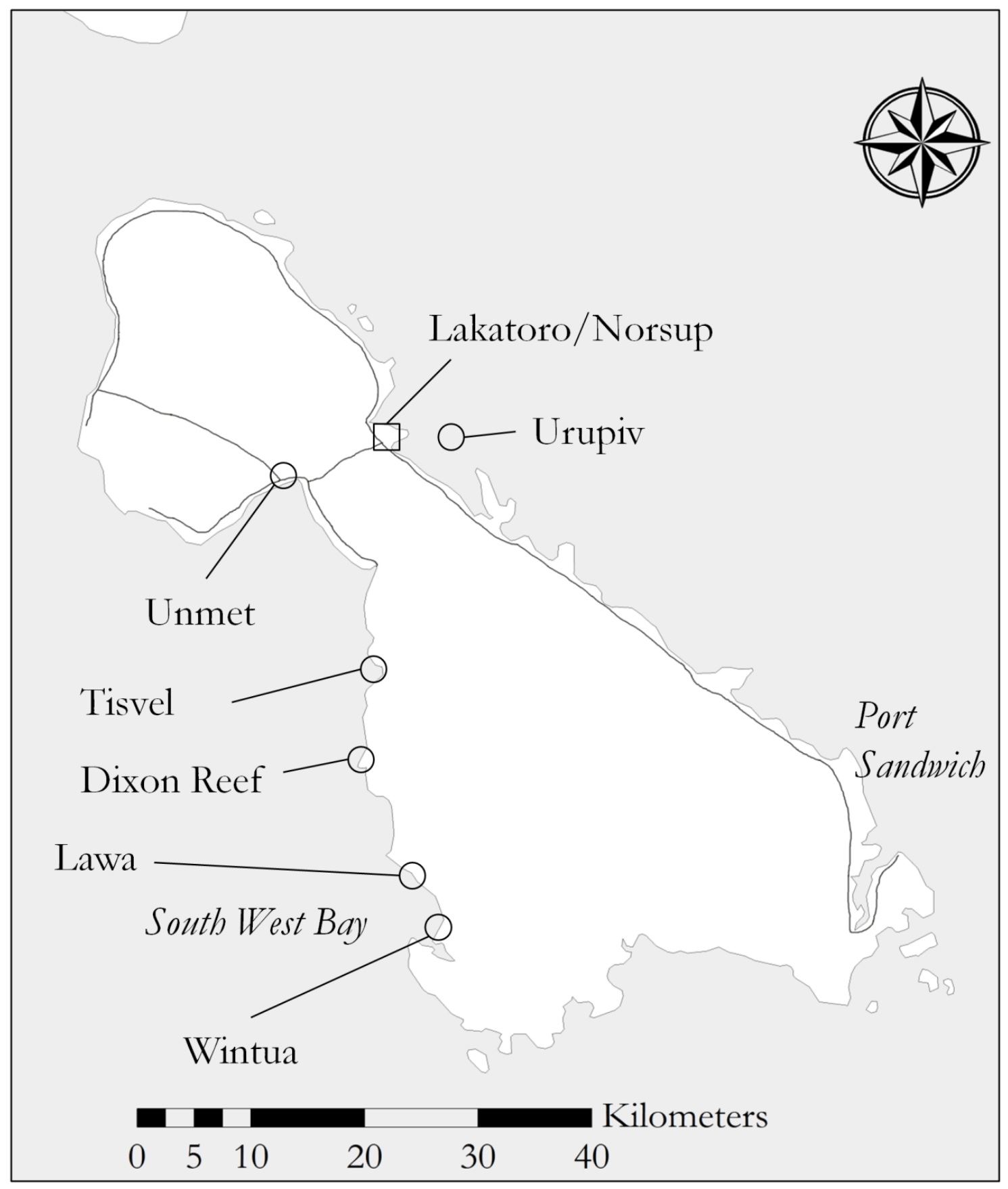

Figure 4.2: Malekula Island, with focus communities

Pre-contact, most of the population lived in the interior, and there were strong distinctions between coastal and interior dwellers (Charpentier 1982). There were trading links present between groups throughout the island, despite the often fierce antagonism between 'saltwater' and the 'bush' peoples: exchange was normally completed via an exchange of women, thus opening a 'rod blong pis' (road of peace) (Huffman 1996). Most Malekulans lived in micro-settlements of 300 to 600 people, based around individual nakamal (dancing grounds) (Charpentier 1982). 
The first contact with Europeans was via Captain Cook, who anchored briefly at Port Sandwich in 1774 and gave the island its name (which according to colloquial accounts is drawn from the French 'mal au cuP). ${ }^{11}$ Malekula suffered massive depopulation during the late $19^{\text {th }}$ and $20^{\text {th }}$ centuries, as witnessed by the stark accounts of the anthropologist Bernard Deacon working in Southwest Bay in 1926: “...intensive study here is impossible - there is no intensive life....you have scarcely an idea of the terrible depopulation here in the last ten years... well, the total number of survivors of the Wilemb district... is twenty two, from forty clans within living memory...there has been a constant gravitation from the interior to the coast, so that there is now not a single village remaining in the whole of Wilemp and Nahate districts, whereas upwards of sixty existed some years ago...depopulation and alcohol sum up the situation." (Deacon 1934: p 20; emphasis in original). Other sources record major outbreaks of dysentery (1905), influenza (1919-1920), and whooping cough (1937 and 1943), all of which had major population-level impacts around the island (Lake 1985).

The establishment of mission stations on the coast at several locations led to large-scale demographic shifts over the course of the $20^{\text {th }}$ century (Lake 1985). Groups from the interior gradually moved to coastal villages, where they were encouraged to become practising Christians. Contact with Europeans slowly increased over the $20^{\text {th }}$ century, with large commercial plantations of coconut being run by French companies (in particular the Caledonian Company of the New Hebrides, now renamed PRV) in the East and North, and missionary activity centred on the central and southern east coast and at Southwest Bay. Tautu, on the east central coast, was the site of major land disputes between PRV and local landowners during the 1960's and 1970's, which influenced the nationalist discourse of the independence era (Van Trease 1987).

\footnotetext{
${ }^{11}$ Literally, 'pain in the arse'
} 
The population of 27,000 (including coastal small islands such as Urupiv, Wala, and Atchin) are now largely based around the coast, and are characterised by exceptional cultural diversity: Lynch and Crowley (2001) estimate that there are 24 languages actively spoken on Malekula, with a further 15 moribund or extinct languages (Figure 4.3). This may make Malekula the most linguistically diverse island, per head of population, in Vanuatu (Crowley 2006a). It is worth noting that whilst these calculations are interesting, they are somewhat subjective: most Malekulans are bi- or tri-lingual, and therefore drawing boundaries between linguistic groups on a map can be difficult (Charpentier 1982). As a result of the earlier demographic declines, an unknown number of small languages have probably disappeared, while others have had their populations dispersed to a number of coastal locations. Most dislocated communities have "...generally retained the ancestral languages in their new locations, while at the small time acquiring an additive competence in the language of their new home communities" (Lynch and Crowley 2001: p 69).

There are two broad cultural divisions, between the V'ënen-taut speaking Big Nambas ${ }^{12}$ in the northwest (3.7.2.2 in Figure 4.3) and the linguistically diverse Small Nambas covering the remainder of the island. Like the rest of Vanuatu, livelihoods on Malekula are based around subsistence farming and nearshore reef harvesting in coastal areas. The commercial and trading centre of the island is Lakatoro/Norsup (the former was British under the condominium, and the latter French), though it has few residents (estimated at 335 in 1999: Vanuatu National Statistics Office 1999).

Lakatoro/Norsup features a bank, general store and hospital, but is largely a transitory setting where people come only to trade.

\footnotetext{
12 'Big Nambas' refers to the style of men's clothing that was unique to this group. While other inhabitants of the island (the 'Small Nambas') traditionally wore a penis-wrapper with the testicles visible, the Big Nambas wore considerably more elaborate attire which completely covered the genitals (Crowley 2006d). These are now no longer worn, except for ceremonial occasions.
} 
Infrastructure on Malekula is limited: a long and potholed road runs along the east coast, stretching round as far as Tenmaru in the northeast and Lamap in the south. Another runs west from Lakatoro/Norsup though the narrowest part of the island, before diverging with one fork ending at the wharf at Lambumbu and the other at Leviamp in the north. There is also a rough, sometimespassable road running over the interior plateau in the northwest between Unmet and Tenmaru. The main airport is at Lakatoro/Norsup, although this is often closed due to factional disputes within the landowning family (McCarter, unpublished field notes). Other airstrips are situated at Lamap in the South East and Wintua in South West Bay, though the latter is often closed for much of the year due to rain. ${ }^{13}$ Outside the areas connected by road, transport is via boat, canoe, or on foot. Coastal shipping provides a major conduit for goods (especially cash crops such as copra, cacao, and kava) and transport between Malekula, Santo to the north, and Efate to the south. Along the west coast of Malekula, services can be extremely irregular at some of the smaller communities. One of the most important developments of recent years has been the extension of mobile phone coverage over most of the island by the Digicel in 2008. Aided by several transmission towers across the island, coverage is now excellent over the much of the coast and most people own and use a mobile phone regularly.

Given the general lack of infrastructure, economic opportunities on Malekula are scarce. Malekulans derive cash income predominantly from the sale of crops such as copra, cacao and kava. However, the sale of these goods is subject to fluctuating prices (driven by both government intervention and global markets) and the infrequent visits of copra boats. A formal commercial occupation is rare, though can include driving transport trucks, running a boat service, commercial fishing, running a small store, or occasional wage labour (primarily on coconut plantations). Only the richest and most commercially-orientated Malekulans do not maintain a personal garden, although there is an increasing reliance on imported food items such as tinned fish and rice. Parallel to the monetary economy is a strong system of ceremony and exchange based around items with high traditional value, such as mats, yams and pig tusks. As noted, this 'kastom economy' is gaining governmental recognition due to ongoing work by the VCC (Regenvanu 2007a); however most Malekulans rely on the cash economy to support payment of school fees, the purchase of imported goods, and the gifting of tithes to the church (McCarter, unpublished field notes).

The relative isolation of most communities also ensures that access to western medical care is infrequent and difficult. The only hospital on Malekula is located at Norsup; however this tends to be only used for the most serious illnesses as transport expenses (particularly from the south of the island) can be prohibitive. There are small clinics in Lawa and Unmet, and aid posts at Tisvel and

${ }^{13}$ Between 2008 and 2010, at the behest of Esmon Sae (a local and, at the time, a government minister) there were efforts to build a road between Lawa and Wintua in SW Bay, in order to transport the limestone needed to make the airstrip suitable for all weather. At last report (November 2010), activity had ceased due to delays, malfunctioning equipment, and rumours of sabotage. 
Dixon Reef for the dispensation of basic medicines. These services commonly lack fundamental medicines such as antibiotics, and visits by trained doctors are rare (McCarter, unpublished field notes).

Accordingly, traditional ethnomedical knowledge remains an important source of healing and recovery for most people. Such remedies are known locally as 'kastom medicine', which is a common term describing remedies that have their origin within the local area. 'Kastom' in this sense does not necessarily refer to 'traditional' medicine with roots in the knowledge of preceding generations; but rather, a medicine that was generated from within (by other ni-Vanuatu, other Malekulans, or other members of the particular community) rather than having been bought by outsiders (McCarter, unpublished field notes). Most Malekulans can self-administer basic remedies for a range of illnesses; however will consult a specialist healer for complex, serious, or unusual illnesses (McCarter, unpublished field notes).

Malekula is one of the better-researched islands in Vanuatu, due to a longstanding fascination from western researchers with its diverse material cultures and a reputation for cannibalism and ritualised male homosexuality (Curtis 2002, see also Herdt 1984). Discussing each of the following works in detail would be outside the scope of my thesis; however see Curtis (2002) for an excellent summary. Two of the most important anthropologists were Bernard Deacon (1934) and John Layard (1942), who predominantly worked in Southwest Bay and Vao and Atchin Islands respectively. The geographer-cum-adventurer Tom Harrison also played a key role in popularising images of Malekula (Harrison 1936). The texts resulting from the work of these anthropologists have proved to be durable, and are still used as the basis for land claims and disputes in their respective areas (Curtis 2002; Geismar 2009; Larcom 1990). Kirk Huffman, the erstwhile curator of the VCC, spent much of the 1970's living with various peoples throughout Malekula, however chose not publish his findings in monograph form (but see contributions to Bonnemaison et al. 1996). More recently, there has been anthropological work conducted amongst the Mbotkot (Funabiki 1981), in South-West Bay (Larcom 1990) and on Tonman Island on the south coast of Malekula (Curtis 1999; Curtis 2002).

There has also been a variety of linguistic research on Malekula, although the languages of Malekula remain relatively poorly-described compared to elsewhere in Vanuatu (Crowley 2006a). The situation is especially urgent given that several languages are moribund and are at risk due to current population patterns and the influence of Bislama (Crowley 2006a). Much of the documentation to be carried out so far has been via the Summer Institute of Linguistics (SIL), ${ }^{14}$ who have produced Bibles in the Urupiv language of the northeast and the Maskelyne Islands in the south (Crowley 2006a).

\footnotetext{
14 A non-profit, Christian organisation which works to provide research, documentation, and materials for the world's endangered languages
} 
Other sources include Tryon (1976), who gathered data for a total of thirteen languages in south Malekula, and Charpentier (1982), who produced a fairly detailed language census of south Malekula during the 1980's. Recently, as part of an intensive effort to document the threatened languages of Malekula led by the late Terry Crowley and John Lynch, there has been linguistic work in several small languages including Aulua (Paviour-Smith 2005), Unua (Pierce 2010), Neverver (Barbour 2011), Nese (Crowley 2006c; Lynch 2005), Naman (Crowley 2006b), Tape (Crowley 2006d), and Avava (Crowley 2006a).

\subsection{Study Sites}

In total, I conducted work in six communities in four areas of Malekula (Table 4.1). The core chapters (Five to Eight) were based in the communities of Unmet, Tisvel, Dixon Reef, and SouthWest Bay (at Lawa for Chapters Five, Six, and Eight; at Wintua for Chapter Seven). A scoping study for this research (described below in Section 4.9) was also conducted at Unmet and Wintua, as well as at additional communities on Urupiv Island. 
Table 4.1: Key characteristics of focus communities on Malekula Island

\begin{tabular}{|c|c|c|c|c|c|c|}
\hline & Urupiv & Wintua & $\begin{array}{c}\text { Lawa (Mahavo } \\
\text { \& Enimb) }\end{array}$ & $\begin{array}{l}\text { Dixon } \\
\text { Reef }\end{array}$ & Tisvel & $\begin{array}{l}\text { Unmet } \\
\text { (Uri) }\end{array}$ \\
\hline Research objectives & $\begin{array}{l}\text { Scoping } \\
\text { study }\end{array}$ & $\begin{array}{l}\text { Scoping study } \\
\text { \& 3(a) }\end{array}$ & $1-3$ & $1-3$ & $1,2,3(a)$ & $1-3$ \\
\hline Population & 443 & 214 & 91 & 180 & 134 & 157 \\
\hline $\begin{array}{l}\text { Approx. years since } \\
\text { village foundation }\end{array}$ & Ancient & 100 & 70 & 60 & 50 & 39 \\
\hline $\begin{array}{l}\text { Approx. hours of travel } \\
\text { to trading centre at } \\
\text { Lakatoro }\end{array}$ & 0.5 & 6 & 6 & 5 & 2 & 1 \\
\hline $\begin{array}{l}\text { Predominant vernacular } \\
\text { language }\end{array}$ & D’Urupiv & Ninde & Ninde & Novol & Avava & V'ënen Taut \\
\hline Colonial language & English & English & English & French & English & French \\
\hline Predominant religion & Presbyterian & Presbyterian & Presbyterian & Catholic & Presbyterian & Catholic \\
\hline $\begin{array}{l}\text { Road access }(0=N o \text {, } \\
1=Y e s)\end{array}$ & 0 & 0 & 1 & 0 & 0 & 0 \\
\hline Clinic $(0=$ No, $1=Y e s)$ & 1 & 1 & 1 & 0 & 0 & 1 \\
\hline $\begin{array}{l}\text { Primary school }(0=\text { No, } \\
1=Y e s)\end{array}$ & 1 & 1 & 1 & 1 & 0 & 1 \\
\hline $\begin{array}{l}\text { Secondary school } \\
(0=N o, 1=Y e s)\end{array}$ & 0 & 1 & 1 & 0 & 0 & 1 \\
\hline $\begin{array}{l}\text { Mean } \% \text { participants } \\
\text { who preferentially speak } \\
\text { Bislama over vernacular }\end{array}$ & $\mathrm{N} / \mathrm{A}$ & $\mathrm{N} / \mathrm{A}$ & 4.08 & 10.52 & 23.26 & 17.02 \\
\hline $\begin{array}{l}\text { Mean monthly visits to } \\
\text { church }\end{array}$ & $\mathrm{N} / \mathrm{A}$ & $\mathrm{N} / \mathrm{A}$ & 4.22 & 6.03 & 2.53 & 6.15 \\
\hline $\begin{array}{l}\text { Mean annual visits to } \\
\text { market }\end{array}$ & $\mathrm{N} / \mathrm{A}$ & $\mathrm{N} / \mathrm{A}$ & 1.65 & 2.39 & 6.05 & 18.91 \\
\hline $\begin{array}{l}\text { Mean number of years } \\
\text { formal education }\end{array}$ & $\mathrm{N} / \mathrm{A}$ & $\mathrm{N} / \mathrm{A}$ & 5.22 & 5.84 & 6.09 & 5.57 \\
\hline
\end{tabular}

Field sites were selected in consultation with representatives of the Malekula and Vanuatu Cultural Centres. For the scoping study in 2008 (Section 4.9), site selection was opportunistic and was based on contact with local VCC fieldworkers during an initial workshop in March 2008 (part of a parallel research program that is not described in this thesis).

For the major research objectives, site selection was carefully stratified according to a number of key characteristics that were identified in the literature as patterning TEK (in Section 2.4.1). Of course, a number of community-level factors may influence the variation of TEK on Malekula, and so attribution of community level variation to any one factor is difficult. However, careful site stratification according to the key variables may corroborate other observed trends in the individual level variation of TEK and allow for robust hypothesis testing (Bernard 1998).

Primarily, the focus communities are situated along a gradient of ease of access to market. This is important because, as noted above, market integration may be one of the key variables to pattern 
TEK within and between communities. In the next chapter, I hypothesise that proximity to market will negatively associate with level of TEK (Godoy et al. 1998; Reyes-García et al. 2005). To test this I selected communities which were broadly similar in other respects: all four are originally language groups from the interior of the island, and were not originally coastal; all four are relatively small (ranging between 91 and 180 inhabitants); and all four have a VCC fieldworker present in the community. In other characteristics I sought to gain an even spread of variance: for example, Dixon Reef and Unmet are Catholic, and thus French-speaking; whereas Lawa and Tisvel are Presbyterian, and thus English-speaking. All four communities belong to a different language group. ${ }^{15}$

\subsubsection{Urupiv}

Urupiv Island the site of scoping research in 2008 (Section 4.9). A small island surrounded by a productive fringing reef, the highest point of Urupiv is no more than a few meters about sea level (Plate 4.2) Vegetation is nearly exclusively plantation mosaic forestry, with some secondary and cultivated woodlands. The inhabitants (who numbered 443 in 1999: Vanuatu National Statistics Office 1999) speak the D’Urupiv language, a dialect of the most widely-spoken language in northeast Malekula (Lynch and Crowley 2001). The widespread distribution of D’Urupiv is largely due to the activities of an early missionary, who utilised it as his language of choice for

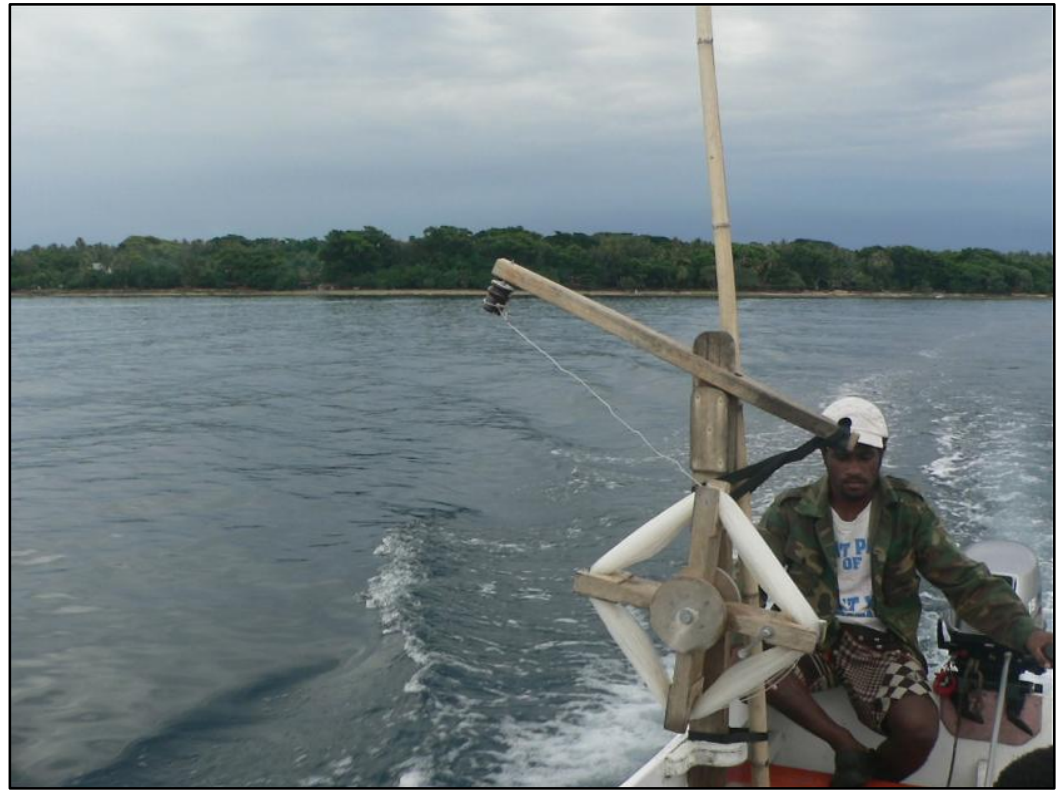

Plate 4.2: Urupiv Island in background (photo by author) spreading the gospel in the area. Urupiv is unique among the focus communities in that it was an original site of settlement before the establishment of the Presbyterian Church on the island.

More recently, Ross McKerras of the SIL, assisted by the Malekula Cultural Centre under Numa Fred Longga (himself a local), created a bible in the local vernacular, and the local Presbyterian

\footnotetext{
15 Throughout this research program I used Bislama as opposed to the local vernacular language. This is reflected throughout the thesis, which does not use local terminology. This is a product of my rapid methodological approach; see Section 3.5 for a discussion on research limitations.
} 
church now runs all services in this language. Inhabitants of Urupiv rely heavily on the reef and marine resources, but most maintain gardens and traditional land on the mainland. Given the proximity to Lakatoro/Norsup (approximately a 20 minute boat ride), there is a high degree of market integration on Urupiv, and many people make the trip to town every day for work or trade. Researchers are relatively common on Urupiv, perhaps given the proximity of the MCC, and there are currently active archaeological digs being run by Stuart Bedford of the Australian National University across the island (McCarter, unpublished field notes). There is a primary school in the village, but no secondary school.

\subsubsection{Unmet}

Unmet was the location of research work throughout the research program, and is the only focus community within the Big Nambas cultural group. The Big Nambas speak V'ënen Taut, and are considered a separate cultural group from other Malekulans because of a number of distinctive cultural traits. Most importantly, the Big Nambas are one of the only groups in Vanuatu to have an ancestral form of hereditary chieftainship, which is still present (though greatly altered from traditional forms). The community in its current form was established around 1972 with one of the last large-scale conversions on Malekula, when the late Chief Virambath purchased a large tract of land and led his people down from Amokh in the interior (McCarter, unpublished field notes). Before this time the land was owned by a Small Nambas group who now live further south. Most inhabitants still maintain strong connections and ownership over traditional lands at Amokh, and openly speak about returning in the near future. The land at Unmet gradually slopes up toward the interior plateau, and is largely covered by coconut plantations or low forest.

The community is large for Malekula, and numbers at least 750 inhabitants (Vanuatu National Statistics Office 1999). My research was largely conducted in the borough of Uri, which was recorded to have 157 inhabitants during the same census. Unmet is connected by road to Lakatoro/Norsup (a trip of around one hour), and there are several trucks in the village which make transport trips every day. As a result, there is a noticeable disparity between elder and younger generations in the community: where several elders speak only V'ënen Taut and rarely visit town, some of the younger generation preferentially speak Bislama and visit the market at least once a week. Being connected with the Catholic Church, the colonial language of Unmet is French. There is a large secondary school in the village, which shares premises with the primary school. The dominant religion remains Catholicism, although there are a number of other denominations including Seventh Day Adventist and Assembly of God. 


\subsubsection{Tisvel}

Located in a small and picturesque bay, Tisvel was the site of fieldwork in 2009 and 2010. A small settlement of 134 people, inhabitants of Tisvel speak Avava. Until recent work by Terry Crowley, Avava was one of the most poorly documented languages in Vanuatu, although this work was only partially completed before his death in 2005 (Crowley 2006a). Tisvel was established in the first half of the $20^{\text {th }}$ century (exact date unknown), when speakers of Avava split into four communities, three of which are on the east coast (Figure 4.4). In consequence, the distribution of the language is now discontinuous, although there are strong links between the four communities and the language remains fairly robust (Crowley 2006a).

Although there is no direct road connection to the Lakatoro/Norsup, access to market is relatively easy: inhabitants at Tisvel commonly walk, canoe, or boat to the road end at Lambumbu, before making the journey of about an hour by truck. There is a regular (three times a week) boat service between Tisvel and the road end. There is no primary school in Tisvel, and most children attend school at Khatbol on the east coast, which they walk across to (around half a day), and

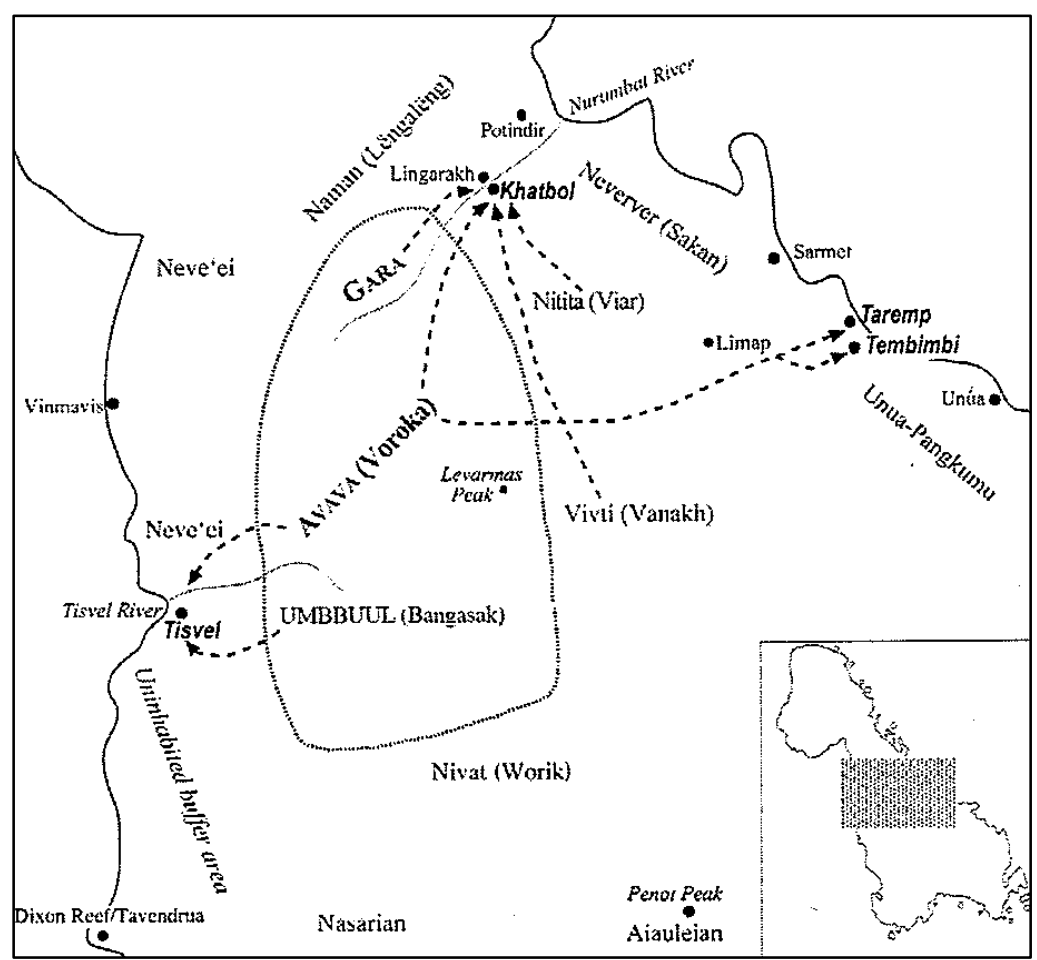

Figure 4.4: Avava-speaking locations past and present (major postcolonial movements indicated by arrows). From Crowley 2006 a. reside there with family during the school term. Tisvel, being a Presbyterian village, has English as a colonial language, although this is not used in any meaningful sense in daily life. Inhabitants maintain regular contact and close links with neighbouring settlements at Vinmavis and Dixon Reef. Tisvel lies in the western rain shadow of the island, and the vegetation is dominated by low dry thickets of Leucaena and Acacia spirorbis.

\subsubsection{Dixon Reef (Tavendrua)}

Dixon Reef (known as Tavendrua in the local vernacular) is situated on a sandy point jutting from the west coast of Malekula, and has a population estimated at 180 (Vanuatu National Statistics Office 1999). This village was the location of work in 2009 and 2010. Dixon Reef is centred around the 
Catholic Church, and correspondingly speaks French as a colonial language. There is a small primary school in the village, but for secondary school youths must go and board elsewhere, commonly at Rensari on the East Coast or at Unmet. Access to Lakatoro/Norsup is more difficult than at the preceding communities, and inhabitants must either canoe or boat to the road end (by boat, around two hours), or walk across the interior to the east coast road. Dixon Reef has a similar vegetation profile to that of Tisvel.

The area was uninhabited before around 1950, when peoples from the linguistic groups of Nasarian and Novol (referred to in Charpentier (1982) as Nabwol) settled the area (Figure 4.5). Nasarian is now moribund with only four elderly male speakers (sadly, one speaker passed away during my stay in the village in 2009). Consequently, the dominant language in the village now is Novol, one of the Lendamboi group of languages that exists in four distinct varieties spread over the south of Malekula (Lynch and Crowley 2001). Inhabitants at Dixon Reef maintain close contact with traditional lands in the interior, including with the Mbotkot language (another of the Lendamboi group of languages).

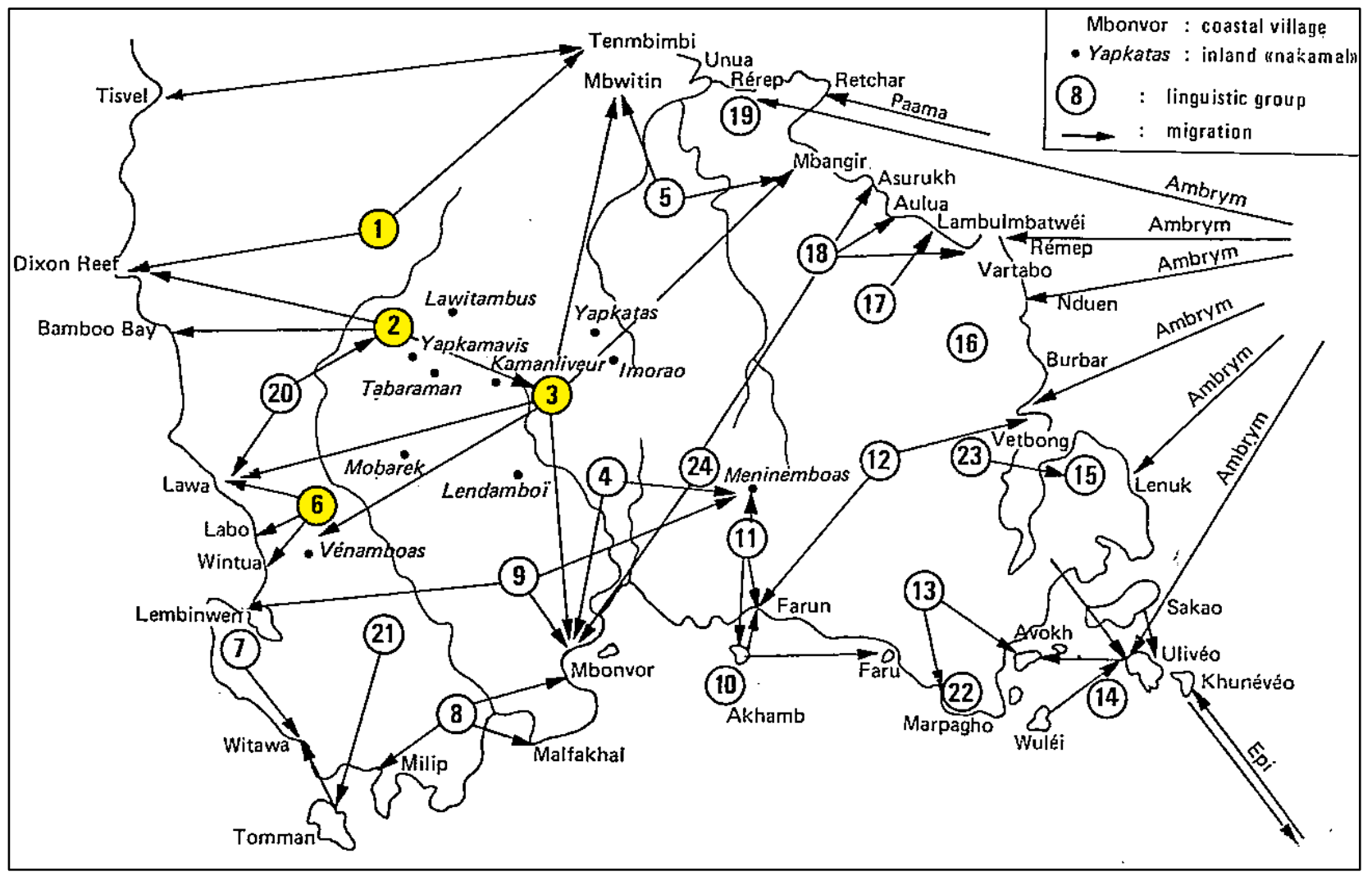

Figure 4.5: Post-colonial population movements in South Malekula. Yellow circles indicate language groups mentioned in text: $1=$ Nasarian; $2=$ Novol (Nabwol); $3=$ Mbotkot; $6=$ Ninde. $20=$ A-vete-ian, an extinct langauge no longer spoken at Lawa. From Charpentier (1982), colour added. 


\subsubsection{South West Bay (Lawa and Wintua)}

Finally, research was undertaken at two communities in South West Bay, Wintua and Lawa. Originally known as Mewun, South-West Bay attained relative fame (at least, to anthropologists) through the scholarship of Bernard Deacon during the 1920's (Deacon 1934). Inhabitants of much of the Bay speak the Ninde language (meaning 'what'), which was originally spoken in relatively close proximity to the coast (although speakers were 'interior' rather than 'coastal' peoples: Figure 4.5). Both villages are surrounded by steep hills dominated by low forest of varying types.

The Presbyterian headquarters for the West Coast of Malekula were established at Wintua around 1920, and began irregularly attracting families from the interior after this point (McCarter, unpublished field notes). However, large-scale movement occurred only after World War Two, when Ninde speakers moved from the interior and founded the villages of Lawa and Labo, as well as joining the established community at Wintua (Charpentier 1982). Because Ninde is a relatively large linguistic grouping, there is a lower rate of bilingualism in South West Bay than elsewhere on Malekula (Charpentier 1982).

There is a large English-speaking secondary school at Wintua which is attended by pupils from around the area, and primary schools in Lawa and Wintua. Access to Lakatoro/Norsup is via an expensive and sometimes arduous boat trip north, which can take up to 6 hours and cost in excess of 6000 vatu (c. $\$ 70$ NZD). There is also a medical clinic based near the school at Wintua, which is usually staffed by trained nurses. Research was conducted in Wintua toward the scoping study (Section 4.9 below) and objective 3(a), and in Lawa (specifically, in the boroughs of Enimb and Mahavo) towards objectives one, two, and 3 (b)..

\subsection{Scoping study: Perceptions of TEK change on Malekula}

TEK and kastom are critical to everyday life on Malekula, as elsewhere in Vanuatu (see Sections 4.3 and 4.4). However, communities on Malekula are subject to the same pressures that may be causing the erosion of knowledge around the country (such as the influence of formal education, as described in Section 4.6), and therefore the loss of TEK remains a topic of high importance on the island (Curtis 2002).

In order to contribute to knowledge around the drivers and nature of TEK change on Malekula, I undertook scoping research in 2008. This research was originally targeted at a Master's degree, and its goal was to assess community perceptions of TEK change, in particular the timing and key drivers of any perceived erosion of TEK. This research utilised a structured interview with 120 participants 
from Urupiv, Wintua, and Unmet (setting outlined in Section 4.8 above; interview schedule included in Appendix 11.10; methods included in Appendix 11.11). I will not fully present these results in this thesis as they do not significantly address the research objectives. However, three key points emerged from this study that I will discuss briefly, as they are useful to contextualise the literature cited above on Malekula and Vanuatu and set the stage for the chapters that follow.

This research resulted in several important findings. First, participants in this study expressed an overwhelming perception that TEK was being lost on Malekula. Indeed, 96\% of participants noted that there was less TEK in their community than in the time of their grandparents (Figure 4.6). This erosion of knowledge was perceived to have happened over two major periods, the first roughly beginning with the arrival of the missionaries and the geographic shift to the coast, and the second between 1970 and 1990 when the formal school system became widespread. Loss of knowledge was perceived to have started earlier on Urupiv (where the first missionaries arrived before 1900) than at Unmet (which was established in 1972).

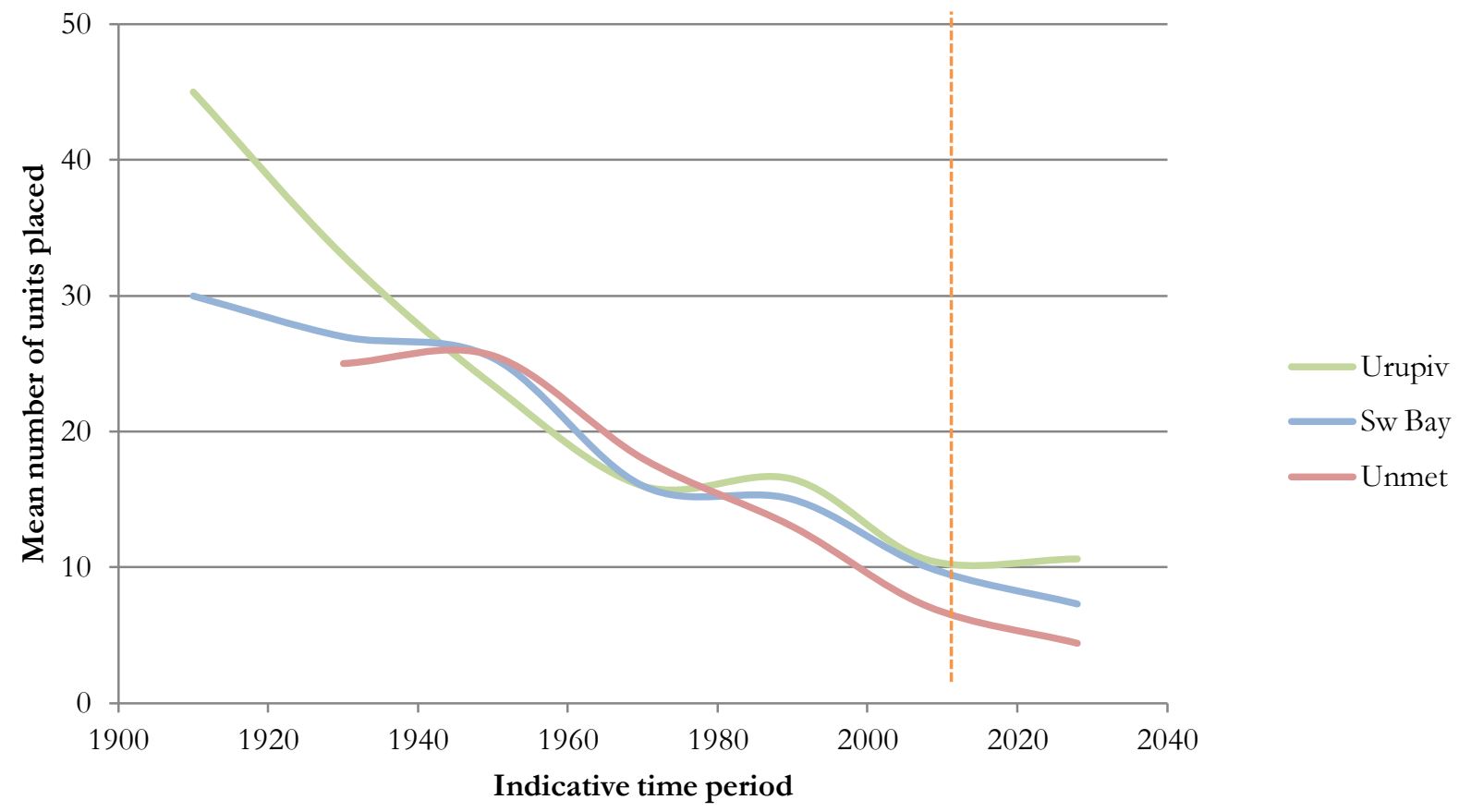

Figure 4.6: Perceived loss of TEK on Malekula (historical and future), utilising trend analysis technique. Dashed line indicates date of study. Y-axis represents number of units placed by participants on time period to represent the amount of TEK at that time (see full methods in Appendix 11.11.3). $\mathrm{n}=120$

Second, interviewees noted several key drivers of TEK erosion (Table 4.2). The most commonlycited drivers were intra-community factors, including the changing values and lack of respect that the younger generation hold for the elders and their traditional knowledge (21.9\% of responses). The second and third most commonly-cited drivers were the church (19.7\% of responses) and the formal school system (16.7\% of responses). At Unmet, participants were more concerned about the 
influence of the church than on Urupiv (Mann-Whitney $U$ tests, $U=256.0, Z=-2.810, \mathrm{p}<.01$ ), ${ }^{16}$ and more concerned about generalised western influence than at Wintua $(U=353.0, Z=-4.517, \mathrm{p}$ $<$.001). Younger participants were less concerned about the influence of the church than older participants (Kruskal-Wallis non-parametric ranking between age cohorts, $\chi^{2}(2)=9.22, \mathrm{p}=.01$; differences confirmed via post-hoc Mann-Whitney U tests).

Table 4.2: Perceived drivers of TEK change on Malekula

\begin{tabular}{lcc}
\hline & $\begin{array}{c}\text { Number of } \\
\text { times cited }\end{array}$ & Percent \\
\hline Intra-community factors & 51 & $21.9 \%$ \\
Church & 46 & $19.7 \%$ \\
Western influence - other & 43 & $18.5 \%$ \\
Formal education & 39 & $16.7 \%$ \\
Population growth & 30 & $12.9 \%$ \\
Market influence & 6 & $2.6 \%$ \\
Inter-community factors & 6 & $2.6 \%$ \\
Don't know & 5 & $2.1 \%$ \\
Other & 5 & $2.1 \%$ \\
Ecological change & 2 & $.9 \%$ \\
Total & $\mathbf{2 3 3}$ & $\mathbf{1 0 0 . 0} \%$ \\
\hline
\end{tabular}

Finally, TEK erosion was perceived to have taken place to differing degrees in different domains and dimensions of knowledge (Figure 4.7). Some domains of knowledge, such as medicinal plant knowledge, were perceived to have only undergone 'small' changes since when the grandparents of interviewees were children. However, others, such as belief in the devils of the bush (aspects of precontact spirituality that often underpinned restrictions on resource use) were perceived to have undergone major changes or to have disappeared altogether. In general, 'knowledge' and 'practice' dimensions of TEK were perceived to be significantly more intact than those of the 'worldview' dimension (Friedman's non-parametric ANOVA, $\chi^{2}(2)=33.91, \mathrm{p}<.001 ;$ confirmed with post-hoc Wilcoxon signed-rank tests).

16 The Mann-Whitney $U$ test is a commonly-used non-parametric statistical test for analysing if one of two independent samples is larger than the other (Field, 2009) 


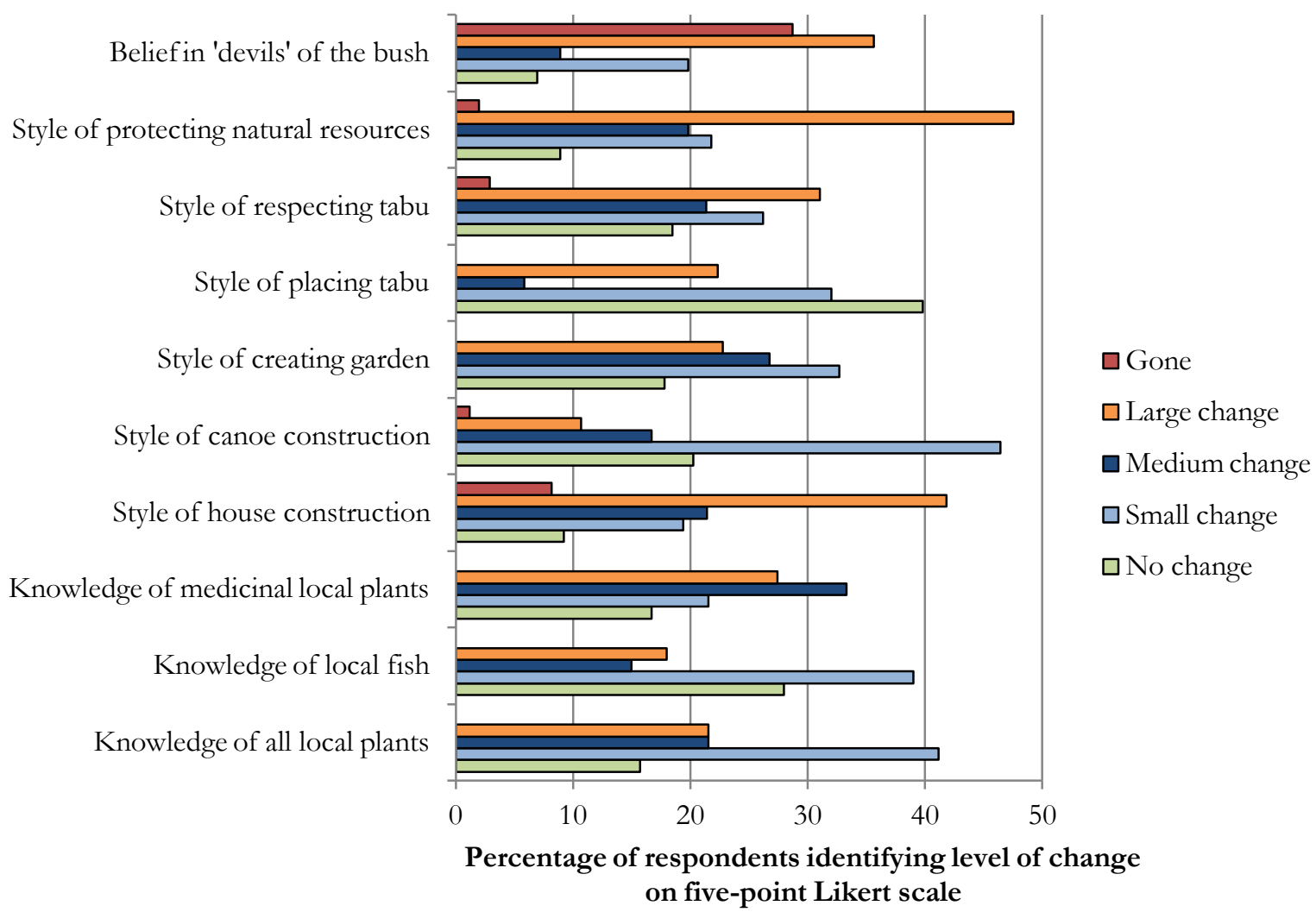

Figure 4.7: Perceived changes in TEK amongst different subject domains. Colours indicate position on five-point Likert scale regarding degree of change. Full methods included in Appendix 11.11.

These data, while they do not form a central facet of this thesis, raise several salient points. Most importantly, TEK erosion was perceived to be a past and current reality by the vast majority of participants in this research. Participants were, in general, concerned about the erosion of TEK and the reduced importance of kastom in everyday life. In describing the formal school and church systems as driving TEK erosion, participants corroborated quantitative data from elsewhere in the world (e.g. Benz et al. 2000; Voeks and Nyawa 2001). However, the importance of the intracommunity factors cited by interviewees highlight the importance of shifts in norms and values of the younger generation in driving TEK erosion. This finding highlights that shifts or erosion in TEK should not be considered as a processes driven entirely by outside actors and influences, and rather that erosion of TEK may represent active choices and changing values amongst younger community members (Zent and Maffi 2009).Variables such as participant norms and values these are rarely factored into models of TEK variation (Reyes-García et al. 2007a).

These findings also show the importance of the geographic shift from the mission to the coast in altering patterns of traditional knowledge and kastom. Participants identified the most significant period of change to be the move to the coast and mission stations at Wintua and Unmet. This is likely to reflect the shift in geographic environment (because the ecology of the coastal environment 
is very different to that in the interior), as well as the influence of Christian teachings and worldviews. The fact that younger participants were less likely to cite the church as a driver of TEK erosion may reflect the fact that, on Urupiv and at Wintua, the church has been woven into contemporary life for at least 60 years, meaning that only the oldest participants would remember it as an extrinsic source of change. The formal school system, however, remains a definitively 'outside' influence today, which is generated and perpetuated outside the island and village life (Niroa 2002). This is corroborated by the finding that participants at Unmet are more concerned about the role of the church in driving TEK erosion, because they have been exposed to the church for the shortest period of time. As such, these data stress that the drivers of TEK change are temporally and spatially variable.

Finally, the finding that erosion of TEK is perceived to be different between domains and dimensions highlights the calls in the literature for an increased breadth of investigation into TEK change (see Reyes García et al. 2007a; or see Section 2.4.1 for a review). As indicated by Figure 2.1, TEK is not a single entity. Rather, it is made up of a number of constituent domains and dimensions, which may respond in differing ways to drivers of social change (Zent and Maffi 2009). This has not often been formally tested, but this point was emphasised by Ladio and Lozada (2004), who found no evidence that theoretical and practical domains of TEK were associated in work in Patagonia. However, most investigations of TEK variation have focussed on a single domain of knowledge, most commonly ethnobotanical knowledge. This remains, then, a clear priority area for future research and is addressed in Chapter Five.

This section has briefly outlined the results of scoping work into community perceptions of TEK change on Malekula. In summary, these findings (while not a key research output of this thesis) contextualise the chapters to come and support some of the key trends from the literature. This scoping study contributed to the remainder of the thesis in two key ways. First, the results detailed here were critical in forming the direction of my hypotheses in Chapter Five (see Table 5.1), as they provided access to relevant data which was based on Malekula Island. Second, this research program armed me with the key contacts, cultural competency, and knowledge of Bislama to continue working on Malekula, and greatly added value to the remainder of the research.

\subsection{Chapter summary}

This chapter has introduced the setting of this thesis in some detail. I began by outlining some historical and geographical characteristics of the Republic of Vanuatu, a globally unique hotspot of biocultural diversity. During this section I also defined the central concept of kastom and outlined the role of the Vanuatu Cultural Centre and their support of TEK and tradition throughout the archipelago. I then detailed Malekula Island, and the focus communities, outlining their unique 
characteristics as well as the procedure through which I selected the field sites. Finally, I discussed the results of scoping research into perceptions of TEK change conducted during 2008, which sets the stage for the core chapters to come.

Having comprehensively reviewed the relevant literature, outlined my approach, and detailed the setting of this research over the past three chapters, I now move on to the core chapters of this thesis. The next chapter investigates the variation of TEK on Malekula, utilising data from a structured interview with 177 participants. The following chapters then investigate the cultural transmission of TEK on Malekula, before examining the maintenance of TEK in the focus communities through both top-down and bottom-up means. 


\section{Explaining variation in TEK on Malekula}

\subsection{Introduction}

Chapters Two, Three, and Four have introduced the literature gaps, methodology, and the setting of this thesis. I now begin to address my core research objectives, as set out in Section 1.3. These objectives are intended to fill the gaps in the literature outlined in Sections 2.4 to 2.7. This chapter addresses objective one ('to examine variation in TEK in four focus communities'), which itself had three parts: to assess TEK variation in relation to socio-demographic explanatory variables (objective 1(a)); to assess evidence of TEK change or erosion (objective 1(b)); and to assess whether different indicators of TEK reveal divergent patterns (objective 1(c)). To deal with these objectives, I utilise a quantitative approach based on structured interview data to assess the variation of individual-level ethnobiological knowledge and practice in four focus communities.

This chapter is built around four key sections. First, I outline a brief summary of the literature, which includes noting some of the work cited above if it is pertinent to describing the research problem. I then present my methods, including discussing the derivation of the dependent and explanatory variables and analytical techniques. I then outline results according to the three sections of objective one, before discussing the findings and implications of this chapter in the context of the wider literature on the subject.

\subsubsection{Background}

Against a background of increasingly complex and intractable global environmental issues, the value of TEK has been commented on at length over the past 20 years (Berkes 2008; Huntingdon 2000; Mascia et al. 2003). In initiatives ranging from biodiversity conservation (Gadgil et al. 1998) to climate change adaptation (Campbell 2006), scholars and practitioners have sought to record, maintain, and utilise TEK. Researchers have noted that TEK represents a source of social capital (Pretty and Smith 2004); can be a vital input into the maintenance of socio-ecological resilience 
(Berkes and Folke 1998; Folke et al. 2007); and may be an effective indicator of biocultural diversity (Maffi and Woodley 2010).

Commentators have hypothesised that TEK is at risk in many areas of the world, and that it can rapidly succumb to the combined pressures of globalisation, environmental degradation, and the “... seductive influence of modernisation and the western worldview" (Voeks and Leony 2004: p S295). Given the value of TEK and the high levels of threat that TEK faces in many locations, a growing and diverse body of literature has sought to quantify and describe individual-level variation in TEK (reviewed in Section 2.4.1). Research has investigated the relationship of TEK to indicators of modernisation, such as years of formal education (Cruz García 2006; Voeks and Leony 2004; Zent 1999), market integration (Godoy et al. 1998; Godoy et al. 2005; Reyes-García et al. 2005), and linguistic erosion (Benz et al. 2000; Reyes-García et al. 2005; Zent 2001); as well as to communitylevel traits, such as settlement nucleation (Ross 2002b), generalised modernisation (Furusawa 2009), or isolation (Reyes-García et al. 2005). Research has also focussed on the ways in which TEK varies with demographic and social variables, such as gender (e.g. Case et al. 2005), birth location (e.g. Voeks and Leony 2004), and age (e.g. Gomez-Baggethun et al. 2010; Ross 2002b).

These data allow us to make broad inferences about ways in which social and environmental change might affect TEK in a diverse range of locations. However, research has generated conflicting results. For example, Benz et al. (2000) and Voeks and Leony (2004) have detailed clear evidence that TEK is eroding under the influence of modernisation in Mexico and Eastern Brazil respectively. However, in work with the Tzeltal Maya in Chiapas, Zarger and Stepp (2004) found evidence of the persistence of TEK despite comprehensive social and economic changes; whilst other work has stressed the complexity of contemporary cultural change (Godoy et al. 1998; Quinlan and Quinlan 2007; ReyesGarcía et al. 2006a). Other research has shown the dynamic nature of TEK by finding that bodies of local ecological knowledge can in fact grow over time (Guest 2002). The dissonance in the results of research to date may be due to a number of conceptual and methodological issues which have hampered the applicability of findings thus far (Reyes-García et al. 2007a; Zent and Maffi 2009)

One key issue is that TEK is conceptually broad, and is made up of multiple cognitive domains. Much of the research on individual-level variation in TEK has focussed on ethnobotanical knowledge, in distinct domains such as knowledge of agricultural varieties (Boster 1986; van Etten 2006), or of wild food resources (Reyes-García et al. 2005). One particularly fruitful line of investigation has been into variation in ethnomedical knowledge (e.g. Begossi et al. 2002; Quinlan and Quinlan 2007; Voeks and Leony 2004). This is an important domain, as up to $80 \%$ of the world's population rely to some degree on traditional herbal medicine, and it forms a discrete and widely salient research focus (King 1996). However, there is little research documenting variation in 
other domains of TEK, such as knowledge of insects or fish; and as such there is every possibility that differing domains of TEK may not display comparable trends (Reyes-García et al. 2007a).

Moreover, TEK covers several dimensions of knowledge, and heuristic descriptions of TEK conceptualise it as a complex system which may encapsulate knowledge, practice, management regimes, social institutions, and a belief system or worldview (see Figure 2.1). Empirically capturing this complexity has proved challenging, although studies have succeeded in differentiating between theoretical and practical knowledge (Reyes-García et al. 2007a). Importantly, these dimensions have been shown to be not always linked. For example, whilst working in Madagascar, Byg and Balslev (2004) found no correlation between theoretical botanical knowledge and the actual extent of plant resource use. As such, there is a need to broaden the scope of ongoing research to capture a wider variety of domains and dimensions of TEK (Begossi et al. 2002; Reyes-García et al. 2007a; Zent and Maffi 2009).

Much of the research noted above indicates that TEK is heterogeneously patterned within communities, commonly along demographic lines, such as gender or age cohort (Case et al. 2005; Voeks and Leony 2004). Actors within these subgroups interact in a wide variety of ways, and this differentiation may be sufficient to produce distinct subcultures and subgroups (Zent and Maffi (2009), see also Boster (1986)). This makes assessments of TEK change difficult, as each subgroup may respond to social and environmental change in distinct ways. For example, younger age cohorts are likely to be particularly vulnerable to acculturation via the formal school system, because in many areas of the world it has become ubiquitous only in the past three decades (Zent and Maffi 2009). Moreover, the dispersal of knowledge within and between communities is complex (Vandebroek 2010).

There are, then, gaps in our understanding of TEK variation within and between communities. In particular, more research is needed into the ways in which variables associated with modernisation or demography may influence individual-level TEK; the factors which may potentially impact on TEK erosion; and comparisons of how different measures of TEK (such as different domains of ethnobiological knowledge) differ in their association with social and economic variables. Filling these literature gaps is critical, as a more nuanced understanding of how TEK varies within and between communities will assist in efforts toward cultural conservation and sustainable development. This is because effective policy and practical responses will ideally be responsive to local conditions and be able to account for and mitigate locally important drivers of TEK change or loss (Zent and Zent 2004). Although the variation of TEK is inherently local and belies generalisation, there are likely to be broad patterns which will be a useful reference point for other case studies at national, regional, and global levels. 
This chapter adds to the literature by examining the distribution of TEK in four communities on Malekula Island, Vanuatu. I make key contributions in three key subject areas: first, by examining a range of modernisation and demographic variables for their impact on TEK; second, by utilising several different domains of knowledge to proxy TEK (richness and diversity of ethnomedical knowledge, knowledge of reef fish, ecological knowledge, and self-reported proficiency), and testing for differences between these different proxies; and third, by examining differences between age cohorts in order to draw some initial conclusions regarding the erosion of TEK. The location of this work in Vanuatu and the application of multi-model inferencing (Section 5.2.6 below) are also novel contributions which add to our knowledge of TEK variation and its analysis (Reyes-García et al. 2007a; Zent and Maffi 2009).

The objectives of this chapter are:

1. To examine drivers of variability in medicinal plant knowledge

2. To examine drivers in the variability of other TEK domains (knowledge of reef fish, ecological knowledge, self-reported proficiency)

3. To assess if different measures of TEK lead to different conclusions about drivers of variability in medicinal plant knowledge

4. To make a specific contribution to examining TEK change in Vanuatu

\subsubsection{Predictor variables}

I derived nine key predictor variables from the literature outlined above to model variability of TEK (Table 5.1). These are broadly grouped into 'demographic' and 'modernisation' categories. I also tested for differences between communities by creating three dummy variables (Lawa, Dixon Reef and Tisvel against Unmet), as there may be other factors, endogenous to the communities, which have not been explicitly measured (such as cultural capacity for TEK). The direction of the hypotheses is locally-specific and is based on pre-interview piloting, the scoping research during 2008 (Section 4.9), and literature from Vanuatu. The justification behind the direction of the hypotheses is explained in text below. 
Table 5.1: Predictor variables for measuring variation in TEK within and between communities

\begin{tabular}{|c|c|c|c|c|}
\hline Group & $\begin{array}{l}\text { Predictor } \\
\text { variable }\end{array}$ & Unit & $\begin{array}{l}\text { Predicted relationship } \\
\text { with dependent variables }\end{array}$ & Exemplar citations \\
\hline \multirow{3}{*}{ 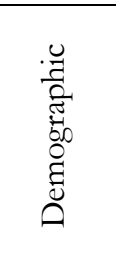 } & Age & Years & Positive association & $\begin{array}{l}\text { (Voeks and Leony 2004; } \\
\text { Zent and Zent 2004) }\end{array}$ \\
\hline & Gender & $0=$ Female, $1=$ Male & No relationship & $\begin{array}{l}\text { (Begossi et al. 2002; Case } \\
\text { et al. 2005; Voeks 2007) }\end{array}$ \\
\hline & Birth locality & $\begin{array}{l}0=\text { Locally born, } 1=\text { Non locally } \\
\text { born }\end{array}$ & $\begin{array}{l}\text { Positive association with } \\
\text { local birth }\end{array}$ & (Voeks and Leony 2004) \\
\hline \multirow{5}{*}{ 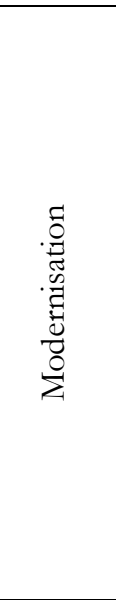 } & $\begin{array}{l}\text { Educational } \\
\text { attainment }\end{array}$ & Years spent in formal education & Negative association & $\begin{array}{l}\text { (Quinlan and Quinlan } \\
\text { 2007; Reyes-García et } \\
\text { al. 2005) }\end{array}$ \\
\hline & $\begin{array}{l}\text { Church } \\
\text { attendance }\end{array}$ & $\begin{array}{l}\text { Number of times attended } \\
\text { church in month prior to } \\
\text { interview }\end{array}$ & Negative association & (Voeks and Leony 2004) \\
\hline & $\begin{array}{l}\text { Linguistic } \\
\text { preference }\end{array}$ & $\begin{array}{l}0=\text { Bislama preferred, } 1=\text { Local } \\
\text { vernacular language preferred }\end{array}$ & $\begin{array}{l}\text { Positive association with } \\
\text { preference for vernacular } \\
\text { language }\end{array}$ & $\begin{array}{l}\text { (Benz et al. 2000; Reyes- } \\
\text { García et al. 2005) }\end{array}$ \\
\hline & $\begin{array}{l}\text { Market } \\
\text { visitation }\end{array}$ & $\begin{array}{l}\text { Number of times visited market } \\
\text { town (Lakatoro) in year prior to } \\
\text { interview }\end{array}$ & Negative association & $\begin{array}{l}\text { (Apaza et al. 2003; } \\
\text { Reyes-García et al. } \\
2005)\end{array}$ \\
\hline & $\begin{array}{l}\text { Commercial } \\
\text { occupation }\end{array}$ & $\begin{array}{l}0=\text { No commercial occupation, } \\
1=\text { commercial occupation }\end{array}$ & $\begin{array}{l}\text { Positive association with } \\
\text { lack of commercial } \\
\text { occupation }\end{array}$ & $\begin{array}{l}\text { (Godoy et al. 1998; } \\
\text { Reyes-García et al. } \\
\text { 2005) }\end{array}$ \\
\hline \multirow{3}{*}{ 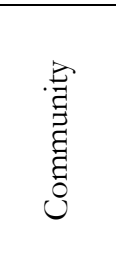 } & $\begin{array}{l}\text { Lawa vs. } \\
\text { Unmet }\end{array}$ & $\begin{array}{l}0=\text { Unmet, Tisvel, Dixon Reef, } \\
1=\text { Lawa }\end{array}$ & $\begin{array}{l}\text { Positive association with } \\
\text { Lawa }\end{array}$ & $\begin{array}{l}\text { (Benz et al. 2000; Ross } \\
\text { 2002b; Zent 2009) }\end{array}$ \\
\hline & $\begin{array}{l}\text { Dixon Reef } \\
\text { vs. Unmet }\end{array}$ & $\begin{array}{l}0=\text { Unmet, Tisvel, Lawa, } \\
1=\text { Dixon Reef }\end{array}$ & $\begin{array}{l}\text { Positive association with } \\
\text { Dixon Reef }\end{array}$ & $\begin{array}{l}\text { (Benz et al. 2000; Ross } \\
\text { 2002b; Zent 2009) }\end{array}$ \\
\hline & $\begin{array}{l}\text { Tisvel vs. } \\
\text { Unmet }\end{array}$ & $\begin{array}{l}0=\text { Unmet, Dixon Reef, Lawa, } \\
1=\text { Tisvel }\end{array}$ & $\begin{array}{l}\text { Positive association with } \\
\text { Tisvel }\end{array}$ & $\begin{array}{l}\text { (Benz et al. 2000; Ross } \\
\text { 2002b; Zent 2009) }\end{array}$ \\
\hline
\end{tabular}

Age: The age of informants has often been associated with variability in TEK, and stemming from the lack of longitudinal studies has commonly been used to infer erosion of knowledge (Voeks and Leony 2004; Zent and Zent 2004). Age is predicted to positively associate with the dependent variables, both because older people have had longer with which to become familiar with their local environment and because transmission of ethnobotanical knowledge on Malekula may have been weakening over time.

Gender: Gender is often strongly associated with varying levels of ethnobiological knowledge.

Research has associated high levels of ethnobotanical knowledge both with women (Caniago and Siebert 1998; Quinlan and Quinlan 2007; Voeks and Leony 2004) and with men (Hanazaki et al. 2000; Kristensen and Balslev 2003; Stagegaard et al. 2002); however it seems likely that the gender dynamics of ethnobotanical knowledge varies across habitats, TEK domains, and forest successional stages (Voeks 2007). Moreover, these dynamics might be expected to vary across cultural groups. Whilst other work from Melanesia (e.g. Case et al. 2005) has noted that males have a higher level of ethnobotanical knowledge, interviews indicated that there should be no difference between men and 
women for the measures of TEK; as such, no association is predicted between gender and all dependent variables.

Birth locality: Migration has been associated with lower levels of ethnobiological knowledge, because length of contact with a given environment can influence individual knowledge of that environment (Guest 2002; Nyhus et al. 2003; Reyes-García et al. 2006a). On Malekula, migration to the focus communities from other areas (largely through an increasing rate of exogamous marriage from other islands) was highlighted as a key driver of linguistic and cultural erosion in the scoping study. Non-local birth is predicted to be negatively associated with all dependent variables.

Educational attainment: Formal education systems are key agents of cultural change and have been associated both positively (Reyes-García et al. 2005) and negatively (Quinlan and Quinlan 2007; Zent 2001), with variation in ethnobiological knowledge. In Vanuatu, it is argued that formal education is negatively impacting on traditional knowledge and culture (Niroa 2002; Regenvanu 2005), and this perception was confirmed at the community level during the scoping study. Therefore, a negative association is predicted between attainment in formal education and all dependent variables.

Monthly church attendance: The spread of Christianity has been the dominant vector of social change in many areas of the world, and is likely to have had a significant impact on ethnobiological knowledge (although to my knowledge this has yet to be empirically tested in the literature). This is particularly true of ethnomedical knowledge, which due to the common links between healing and indigenous spiritualties has been strongly discouraged by the churches (Caniago and Siebert 1998; Voeks and Leony 2004). The scoping study identified the church as a key vector of cultural change, so degree of affiliation with the church (proxied by monthly church visits), is predicted to negatively associate with all dependent variables.

Linguistic preference: Competence in vernacular language has been shown to strongly associate with high levels of ethnobiological knowledge, in part because vernacular languages often encode deep and locally-based environmental knowledge (Harrison 2007; Nettle and Romaine 2000). Conversely, use of a foreign or colonial language has been shown to associate negatively with TEK (Reyes-García et al. 2005). On Malekula, some participants (mostly younger) preferentially use Bislama in the village environment over the local vernacular; this tendency is predicted to negatively associate with all dependent variables.

Market visitation: Market integration is thought to be a key potential driver of erosion of ethnobiological knowledge, because it can induce specialisation into marketable domains or serve as a conduit for the introduction of outside norms and worldviews (Quinlan and Quinlan 2007; Reyes- 
García et al. 2005). Research has shown, however, that this trend is complex, and is dependent on the type of market influence (for example, non-timber forest products or sale of agricultural goods) and the domains of knowledge being surveyed (Godoy et al. 1998; Reyes-García et al. 2007b). Here, number of visits to market in the past calendar year is proxied with degree of market integration, which is predicted to negatively associate with all dependent variables. I note that this variable is, by necessity, based on participant estimates; and whilst these are likely to be accurate for those with few visits to market, there may be some recall bias for those with many visits. Because few participants recorded over 10 visits to market in the past calendar year I do not anticipate that this problem is significant.

Commercial occupation: The presence of a formal commercial occupation may reduce the time available for the use and collection of ethnobiological knowledge and skills, or may result in specialisation in one narrow, commercially viable, domain (Godoy et al. 1998). Whilst a formal commercial occupation is not common on the West Coast of Malekula, opportunities in transport, fishing, and selling of local goods such as kava are present and some participants define themselves as wholly occupied by these occupations. Employment in a commercial occupation is predicted to negatively associate with all dependent variables.

Community: Each community encapsulates a number of unique factors, meaning that it is difficult to demonstrate that community-level differences are contingent on any one particular variable. However, as noted, the four focus communities are situated along a clear gradient of ease of access to market (Section 4.8), and it is hypothesised that this will impact on community-level knowledge in each village. Therefore I predict that Unmet, which has the easiest access to market of the four (a direct road connection, c. $1 \mathrm{hr}$ ), will have the lowest levels of TEK. To test this I created three dummy variables (each community vs. Unmet).

\subsection{Methods}

\subsubsection{Setting}

This research was based in the focus communities of Unmet, Tisvel, Dixon Reef and Lawa (Table 4.1). Please see Section 4.8 for more information on study sites and site selection.

\subsubsection{Sampling}

The instrument for data collection for this chapter was a structured interview, which was piloted with 18 expert informants across all four communities to ensure intelligibility and salience of questions. In total, 177 structured interviews were undertaken, comprising of multiple freelists, an ecological 
knowledge quiz, and collection of socio-demographic information (Appendix 11.9). Interviews were conducted in Bislama. All interviews with female participants were undertaken by trained local female research assistants.

Participant selection was stratified by age class (broadly divided into three classes of 18-30, 31-60, and $60+$ ) and gender, as these variables have been implicated in patterning TEK within communities (Case et al. 2005; Reyes-García et al. 2006a; Voeks 2007; Voeks and Leony 2004; Zent and Zent 2004). In surveys of this kind participant selection would ideally be fully randomised, based on a priori household data, in order to reduce bias and allow the extension of results over the entire population (Zent and Maffi 2009). However, this data was not available, and so within the stratifications participant selection was opportunistic and reliant on who was available on the day of the interview. As such, this was not a random sample of the community, though I aimed for $30 \%$ coverage of households in each community. This process was assisted by representatives from the VCC and local informants. Sample stratification was partially successful (Table 5.2), although it was difficult to attain sufficient sample sizes in the $60+$ age cohort.

Table 5.2: Participants in structured interview on TEK variation

\begin{tabular}{lcccccc}
\hline & \multicolumn{3}{c}{ Age cohort } & \multicolumn{2}{c}{ Gender } \\
& & $18-30$ & $31-60$ & $60+$ & Male \% & Female \% \\
\hline Total & $\mathbf{1 7 7}$ & $\mathbf{5 9}$ & $\mathbf{9 0}$ & $\mathbf{2 8}$ & $\mathbf{5 3 . 1}$ & $\mathbf{4 6 . 9}$ \\
Lawa & 49 & 10 & 27 & 12 & 49.0 & 51.0 \\
Dixon Reef & 38 & 17 & 15 & 6 & 63.2 & 36.8 \\
Tisvel & 43 & 18 & 22 & 3 & 51.2 & 48.8 \\
Unmet & 47 & 14 & 26 & 7 & 51.1 & 48.9 \\
\hline
\end{tabular}

All data collection strictly adhered to the ethical procedure described in Section 3.3.2. Full prior informed verbal consent was obtained from all research participants, and all interviews remain confidential. This research was approved by the Human Ethics Committee at Victoria University of Wellington (approval number 16500) and the Vanuatu National Cultural Council under their Cultural Research Permit program.

\subsubsection{Variability in medicinal plant knowledge}

Ethnomedical data were collected through the use of freelisting, a widely-used structured interview technique (Bernard 2005; Lykke et al. 2004; Medley and Kalibo 2004; Quinlan 2005). In freelist interviewing participants are asked to list items within an emic category or cultural domain, giving a rapid and simple appraisal of a participant's knowledge of a particular subject. Utilising this technique, I infer conclusions about an individual's total knowledge within a domain based on the assumption 
that more knowledgeable individuals will list more than less knowledgeable individuals (Quinlan and Quinlan 2007).

Accurate freelists require that the domain being tested is narrow and well-defined, necessitating a piloting phase to determine suitable emic categories. I adapted a two-stage freelisting technique from Quinlan and Quinlan (2007) to account for the large size of the medicinal plant knowledge domain. First, I purposively selected a sample of 18 expert informants (comprising 11 men and 7 women), all of whom were identified from within the community as being knowledgeable in kastom medicine. I asked the pilot sample to list "....all the illnesses that are treated in this community using kastom medicine" (Appendix 11.9.1).17

In total, 72 illnesses were cited (see Table 5.3 for the 30 most salient). I calculated the saliency (or Smith's S) ${ }^{18}$ of each of the illnesses and selected the ten most salient. Additional experts confirmed the validity of these illnesses in each community. In particular, I confirmed that each of the ten illnesses could be expected to be known by a wide cross-section of the community, rather than being restricted to specialist healers.

Table 5.3: 30 most salient illnesses derived from initial freelisting with 18 expert informants

\begin{tabular}{lcc|lcc}
\hline Illness & $\begin{array}{c}\text { Frequency of } \\
\text { mention }\end{array}$ & Salience & Illness & $\begin{array}{c}\text { Frequency } \\
\text { of mention }\end{array}$ & Salience \\
\hline Headache & $\mathbf{1 4}$ & $\mathbf{0 . 6 0}$ & Epilepsy & 3 & 0.11 \\
Cough & $\mathbf{1 2}$ & $\mathbf{0 . 4 9}$ & Constipation & 4 & 0.10 \\
Diarrhoea & $\mathbf{7}$ & $\mathbf{0 . 3 6}$ & Sore stomach & 4 & 0.08 \\
Earache & $\mathbf{9}$ & $\mathbf{0 . 3 2}$ & Sores & 3 & 0.08 \\
Toothache & $\mathbf{7}$ & $\mathbf{0 . 3 0}$ & Aching heart & 3 & 0.07 \\
Cuts and sores & $\mathbf{6}$ & $\mathbf{0 . 2 8}$ & Boils & 2 & 0.07 \\
Arthritis & $\mathbf{6}$ & $\mathbf{0 . 2 5}$ & Sore ribs & 3 & 0.07 \\
Malaria & $\mathbf{5}$ & $\mathbf{0 . 2 2}$ & Asthma & 2 & 0.06 \\
Hepatitis & $\mathbf{5}$ & $\mathbf{0 . 2 1}$ & Snek spirit & 3 & 0.06 \\
Conjunctivitis & $\mathbf{5}$ & $\mathbf{0 . 1 7}$ & Hernia & 2 & 0.06 \\
Sore body & 4 & 0.14 & Ringworm & 3 & 0.06 \\
Impotence & 5 & 0.13 & Nausea & 3 & 0.06 \\
Fever (general) & 4 & 0.12 & Dengue fever & 2 & 0.05 \\
Poison & 4 & 0.12 & Female infertility & 2 & 0.05 \\
Sore urination & 3 & 0.11 & Sprain & 1 & 0.05 \\
\hline NB: Ten most salient illness domains listed in bold typeface &
\end{tabular}

The ten most salient illnesses were then included in the structured interview. All participants were asked to "...list all the kastom medicine you know to treat [each illness]". 20

\footnotetext{
17 In Bislama: "Euri sik we yufala long [village name] stap lukaotem wetem ol difren kaen kastom meresin"

${ }^{18}$ Smith's $\mathrm{S}$ is a measure of cognitive and cultural significance that weights the frequency of responses inversely by the rank of that item in each list. See Smith (1993) for more detail.

19 An illness with traditional aetiology - literally, an invasion of the body by a malicious 'snake spirit'. Commonly characterised by fevers, headaches, and lethargy.
} 
Each plant cited through this procedure was identified by its name in the local vernacular, or in Bislama if the former was not known by the participant. Voucher specimens of each plant were collected and identified with the assistance of botanists at the Vanuatu Department of Forests. All specimens are deposited at the National Herbarium of Vanuatu in Port Vila. 89\% of the plants collected were identified at least to genus level, however the remainder could not be identified due to poor specimen condition, or because they were unable to be found by the guides assisting collection. Unidentified plant data are included in the analyses below; as such, my conclusions are based on folk species, which do not always coincide with scientific species. In recognition of agreements with the VCC aimed at protecting the intellectual property of informants, species names of medicinal plant specimens are not listed in this or other publications.

\subsubsection{Supplementary measures}

As noted above, TEK is most commonly proxied with ethnobotanical knowledge. In this research I also took three further measures of TEK in order to observe variation in TEK across domains.

\subsubsection{Knowledge of reef fish}

As noted, all of the focus communities are relatively recent arrivals on the coast. As such, I might hypothesise that they do not have the in-depth cultural knowledge of the inshore marine environment that has been noted in other island communities in Vanuatu (Hickey 2006b). However TEK is dynamic and I would expect this knowledge to be gained with increased time of residence at the coast.

To examine individual and community-level variation in knowledge of reef fish I conducted another freelisting exercise, as described above. I developed an initial question which asked participants to "...list all the fish you know that live on the reef in this village." 21 Piloting with the same sample of 18 expert informants revealed that the freelist question was sufficiently narrow and well-defined, and this was included in the final interview.

I did not identify fish species using scientific species names, and as such focus only on abundance of citations of folk species. As noted, these may not correspond with scientific species.

\subsubsection{Ecological knowledge quiz}

To triangulate freelist data I used an 18-question ecological knowledge quiz, following Godoy et al. (1998). The quiz comprised of six photo identifications (taken by me and identified with the

\footnotetext{
${ }^{20}$ In Bislama: "Evri kastom meresin we yu save blong lukaotem [name of illness]

${ }^{21}$ In Bislama: "Evri fish we yu save se I stap long rif long ples ia"
} 
assistance of botanists at the Vanuatu Department of Forestry and Wheatley (1992)); three bird identifications (from Bregulla 1992); two shellfish identifications (from Allen and Steene 1994); five fish identifications (from Lieske and Myers 1999); and three questions testing knowledge of ecological interactions (devised with the assistance of the Vanuatu Cultural Centre).

All species were locally present, and I included questions of varying difficulty in order to provide variance. Correct vernacular answer names were given by VCC fieldworkers and cross-checked with expert informants. Answers were marked as correct only if the interviewee knew the correct name of the species concerned in the local vernacular language. Percentage score in the quiz forms the dependent variable. The full quiz is included in Appendix 11.9.

\subsubsection{Skill proficiency}

I derived 18 key ethnobiological skills in five domains from group exercises with a variety of informants (see Section 6.2.4 for a detailed methodology on the derivation of key skills). For each key skill participants were asked to self-rate their own proficiency using a sevenpoint Likert scale ranging from 'no proficiency' to 'expert'. In order to make explanation of the Likert scale more intelligible to participants I used a stick (c. 1 meter long) equally divided into seven parts (Plate 5.1): the lowest reflected 'no proficiency', while the highest represented

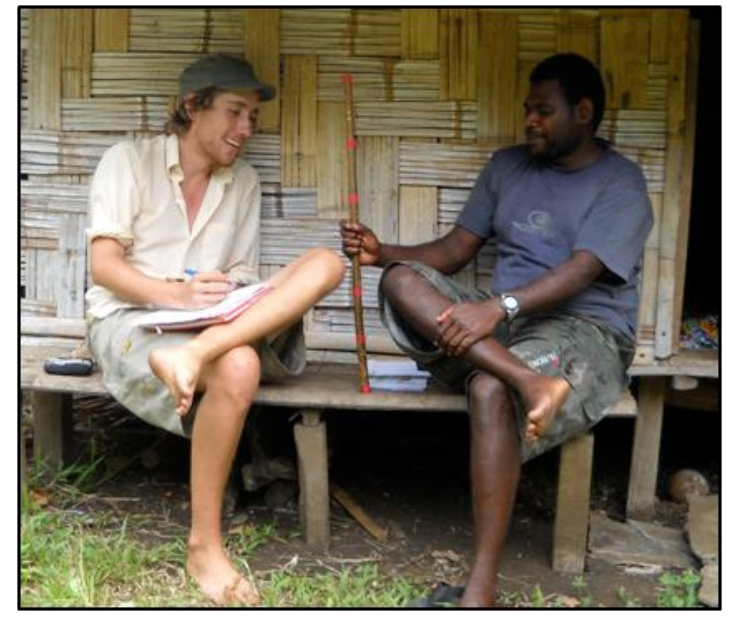

Plate 5.1: Interviewing informant using Likert scale (photo by author) 'expert'.

\subsubsection{Analysis}

\subsubsection{Dependent variables (measures of TEK)}

Six key dependent variables were derived from the methods described above (Table 5.4). The first three (richness of medicinal plant citations, individual Shannon index, and group-level Shannon index) pertain to the data gathered during the freelists on medicinal plant knowledge. The last three pertain to the supplementary measures of TEK described above. Explanation of the derivation of these dependent variables is provided below, where necessary. 
Table 5.4: Summary table of dependent variables

\begin{tabular}{|c|c|c|c|c|}
\hline Variable & Method of analysis & Range & Mean & SD \\
\hline Richness of medicinal plants cited & Multiple regression via AIC & $1-39$ & 10.06 & 6.15 \\
\hline Shannon index (individual) & Multiple regression via AIC & $0.69-3.6$ & 2.1 & 0.61 \\
\hline Shannon index (group-level) & $\begin{array}{l}\text { t-tests and rarefaction } \\
\text { curves between groups }\end{array}$ & $\mathrm{N} / \mathrm{A}$ & $\mathrm{N} / \mathrm{A}$ & $\mathrm{N} / \mathrm{A}$ \\
\hline Abundance of reef fish citations & Multiple regression via AIC & $0-42$ & 11.51 & 6.78 \\
\hline $\begin{array}{l}\text { Percentage score in ecological } \\
\text { knowledge quiz }\end{array}$ & Multiple regression via AIC & $16.67-100$ & 60.51 & 17.93 \\
\hline $\begin{array}{l}\text { Individual proficiency score across } 18 \\
\text { key skills }\end{array}$ & Multiple regression via AIC & $3-15$ & 9.17 & 2.73 \\
\hline
\end{tabular}

\subsubsection{Richness and abundance}

Freelists can be used to generate a number of measures of TEK, the most obvious being the sheer number of items cited per participant (Quinlan 2005). Here I distinguish between citation abundance, or the number of citations recorded per participant; and citation richness, or the number of distinct folk species recorded per participant. This distinction is important: if one participant cites 30 distinct species, they clearly have a more diverse knowledge base than a person who cites three plants 10 times each.

For medicinal plant knowledge, individual level richness and abundance were very highly correlated $\left(r_{s}=0.990, p<.0001\right)$, and when plotted it was clear there were no significant outliers. As such, I tested only citation richness in most of the following sections. For the freelist on knowledge of reef fish, I do not have data on citation richness (having not identified fish using scientific names), and so focus on citation abundance of folk species only.

\subsubsection{Shannon index}

The application of methodology from ecology to ethnobiology has become more popular in recent years (Begossi et al. 2002). In particular, the value of using diversity indices (such as Shannon or Simpson's) to assess the diversity of individual and population-level knowledge has been recognised in several ethnobiological studies (Akerreta et al. 2007; Begossi et al. 2002; Benz et al. 2000; Hanazaki et al. 2000; Ladio and Lozada 2004). In this chapter I utilise the Shannon index, which takes into account both richness and evenness (relative abundance) to assess individual and population-level variation in knowledge (Begossi 1996). It is an effective tool with which to qualify the information gleaned through examining richness and abundance of citations: instead of simply analysing how many medicinal plants participants know, it allows one to assess variation in what kinds of plants they are able to cite. This allows objective comparisons between communities or in categories within communities. 
Calculations are made through the formula $H^{\prime}=\Sigma p_{i} \log p_{i}$, where $p_{i}$ is the proportion of the individuals of the ith species, here the number of informants per species. I calculated both individual and group level (using groupings based on community or on demographic data such as sex and age) diversity indices. Although individual-level H' (the diversity of each participants' citations) forms a key dependent variable, group level analysis may reveal different patterns because species richness may vary significantly between groups. Categorical differences were tested for significance using a modified t-test (Magurran 1988).

\subsubsection{Species accumulation curves and rarefaction}

I also plotted rarefaction curves, which are used to estimate the total richness (in terms species of medicinal plants known) of $N$ individuals from the pooled total species richness, after randomising the sample order (Gotelli and Colwell 2011; Williams et al. 2007). These can be plotted with 95\% confidence intervals, which allow us to determine whether species richness is significantly different between groups (Williams et al. 2007). Because species citations were randomly and sequentially recorded after one another during the interview process, I computed individual-based rarefaction (using the terminology of Gotelli and Colwell 2001) and derive Mao-Tao rarefaction curves based on formulae in Colwell (2009).

Rarefaction curves illustrate the rate at which new species are cited, but they do not reveal the total richness of the site - in this case, the total richness of the community's medicinal plant knowledge if sampling is not sufficiently thorough (Magurran 2004). If this is the case, then the curves will continue to show an upward trend illustrating the extent to which sampling effort might have to be increased to reveal more species. One means to assess sampled richness against the estimated richness of the total site is to plot rarefaction curves against non-parametric richness estimators, or against asymptotic functions such as the Michaelis-Mentin algorithm (Colwell et al. 2004; Williams et al. 2007). In recent work in South Africa, Williams et al. (2007) show that the first order Jackknife estimator (Jack1) most accurately approximated known richness of a large ethnobotanical sample, and that the Michaelis-Mentin Means (MMMeans) estimator was also promising. As such, here I plot both against the rarefaction curves described above.

\subsubsection{Proficiency score in 18 key skills}

I intended to use mean or median score from the seven-point Likert scale score as an indication of overall proficiency over the 18 key skills. However, this became problematic after it became clear the scale was only partially understood by some participants. These participants (a rough estimate, based on field notes, is around 25\%) would simply note the highest rank (7 on the scale, indicating 'expert') for all skills they knew and 0 for all they didn't. 
As such, I believe that the scale does not provide an accurate measure of skill proficiency over the 18 skills. It does, however, give an indication of which participants had some experience at each skill, regardless of actual proficiency. To gain a measure of this I collapsed the seven-point scale into a binary code of 'some proficiency' (1) and 'no proficiency' (0). These scores were then summed across all 18 skills for each participant to derive a 'proficiency score' for each participant.

The proficiency score is more fully discussed in Chapter Six. At this stage, I note that ten of the 18 skills were gendered (five only being conducted by males, and five only by females). This potentially confounds the proficiency score, as if either the male or female skills were significantly more difficult this would bias the proficiency score toward each gender. I tested for differences between mean scores for male-only and female-only skills, and found that while scores were slightly higher in the female-only skills, this difference was not significant.

\subsubsection{Exploring the dependent variables}

I explored the hypotheses outlined in Table 5.1 in two phases. First, I used bivariate analysis to test each of the variables separately and observed if the specific hypotheses relating to that variable were upheld. Where the distribution of dependent variables differed significantly from normal, Spearman's rank correlations were used (all dependent variables except percentage score in ecological knowledge test); where this was not the case Pearson's correlation coefficients were used (Field 2009).

Second, I developed multiple regression models to test relationships between the key dependent variables and the demographic, modernisation, and community predictor variables. This step is necessary because individual-level knowledge will be shaped by a number of factors, and each of the predictor variables outlined above will not work in isolation. However, selecting the variables for inclusion in each model requires some care, because the inclusion of surplus predictor variables can cause information loss (Burnham and Anderson 1998).

To select between different models (or combinations of predictor variables) I used multi-model inferencing, as developed by Burnham and Anderson (1998). This process uses the strength of the available data and the principle of parsimony to derive a 'best model' subset which can then be tested (Burnham and Anderson 1998; Burnham and Anderson 2004). Multi-model inferencing is grounded in the Information Theoretic approach, and compares models using the Akaike Information Criterion (AIC) and akaike weights (AIC.w). I do not discuss the derivation of AIC values here (see Burnham and Anderson (1998) or Lavoue \& Droz (2009) for an applied example); I note though that they are based on Kullback-Leibler information, and allow assessment of the best approximating model. To correct for small sample size I use $\mathrm{AIC}_{\mathrm{c}}$, and derive $\Delta \mathrm{AIC}_{\mathrm{c}}$ in order to delineate models with the 'best model' subset (all models within $2 \Delta \mathrm{AIC}_{\mathrm{c}}$ ). 
Table 5.5 displays a series of individual models which are based on combinations of the predictor variables outlined above. I use the AIC procedure to find the 'best' model subset for each of the dependent variables, and thus choose which model is most appropriate for the data. Models one to four represent the main groups of predictor variables. Models five to eight test combinations of demographic and modernisation predictors which have been found to be important in other research (see references in Table 5.1). Models nine and ten specifically test market influence, based on the selection of communities along a gradient of ease of access to market. Finally, model 11 tests for differences between communities whilst accounting for gender.

Table 5.5: Eleven basic models for testing via AIC analysis

\begin{tabular}{cl}
\hline Model & Variables included \\
\hline 1 & All variables \\
2 & All demographic variables \\
3 & All $\underline{\text { modernisation variables }}$ \\
4 & All community dummy variables \\
5 & Age, gender \\
6 & Age, gender, education \\
7 & Age, gender, education, linguistic preference \\
8 & Age, gender, education, linguistic preference, market visitation \\
9 & All community dummy variables, market visitation \\
10 & All modernisation \& $\underline{\text { community dummy variables }}$ \\
11 & Gender and community dummy variables only \\
\hline
\end{tabular}

Before multiple regression modelling I utilised standard diagnostic tests and plots to ensure the assumptions of regression analysis were met. None of the dependent variables were strongly correlated $\left(r / r_{s}<0.7\right)$. For the regression analyses, medicinal plant citation richness was logtransformed and reef fish citation abundance was square-root transformed in order to ensure normality.

Descriptive statistics, bivariate correlations, and multiple regression were calculated using SPSS 17.0 for Windows (SPSS Inc. 2008). Diversity indices were calculated from raw data using the Shannon Index calculator from Chang Bioscience (Chang Bioscience 2011). All rarefaction curves were calculated using EstimateS 8.2 software (Colwell 2009). Mao-Tao rarefaction curves and their confidence intervals are computed analytically, and so they do not require any re-sampling runs in EstimateS. To compute Jack1 and MMMeans estimators the sample order was randomised 100 times for each sample accumulation level, following the methodology of Williams et al. (2007). 


\subsection{Results}

Results are presented according to the objectives set out in the introduction. First, I present the data on medicinal plants (including bivariate correlations and multivariate regression), before outlining similar analyses of the three supplementary measures of TEK.

\subsubsection{Medicinal plant knowledge}

Freelists inventoried 262 folk species of plant from 73 families and 180 genera, of which 237 were identified at least to genus level. A complete list of plant families cited during interviews is included in Appendix 11.12. The remaining 26 citations were either uncollectable (i.e. restricted to hill-country habitats out of reach of this survey) or remained un-identified due to poor specimen condition. Participants also listed numerous remedies that were either non-herbal or modern in origin (for example, cooking oil or paraffin). As the freelist question targeted kastom medicines only, these data were not considered.

The most important families in terms of number of species represented across freelists for all ten illnesses were Fabaceae (20 species), Euphorbiaceae (15 species), Malvaceae (13 species), Moraceae (11 species), and Poaceae (10 species). In terms of number of citations, the most important families were Asteraceae (197 citations), Arecaceae (130 citations), Euphorbiaceae (126 citations), Malvaceae (110 citations), and Moraceae (109 citations), which between them accounted for 34\% of the total citations. In total, the top ten most commonly cited families accounted for $51 \%$ of citations.

$27.3 \%$ of species cited were commonly cultivated in the village environment or surrounds, and on average, $48.6 \%$ of a participant's citations were cultivated. Trees were the most commonly cited life form, followed by herbs and vines (Figure 5.1). 


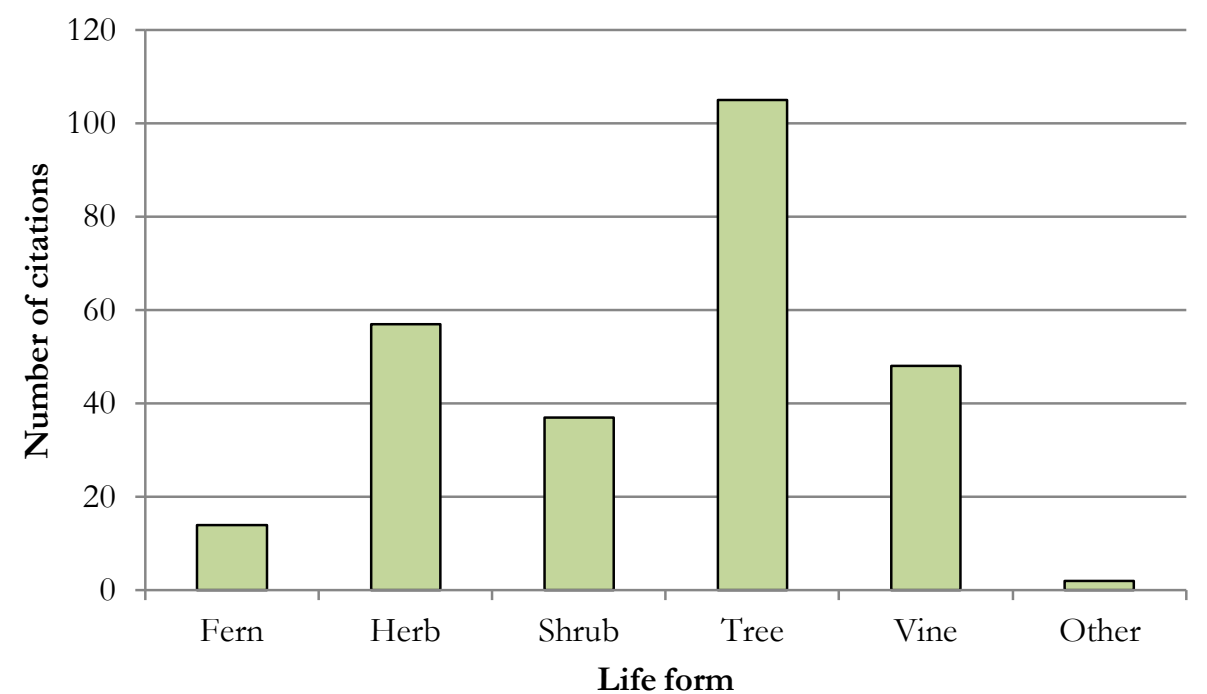

Figure 5.1: Number of citations per life form

\subsubsection{Bivariate correlations and explorations}

Richness and diversity $\left(\mathrm{H}^{\prime}\right)$ of species cited was, in general, highest in the most salient illness (Table 5.6). This pattern was not reflected in abundance of citations, however, which were the highest for the domain of cuts and abrasions. This may reflect the ubiquitous nature of one treatment for cuts, a common invasive vine in the Asteraceae family, which was cited by $70.1 \%$ of all participants. As such, evenness, or relative abundance, of citations was noticeably lower for this illness domain, and for malaria. Conversely, evenness was extremely high for the illness domains of headache and arthritis, indicating a low level of dominance of any one plant species.

On an individual level, the majority of participants knew multiple kastom medicines, although two individuals were not able to cite medicines for any of the illnesses. Most individuals cited six plants across the 10 freelists (the mode), and the mean number of plants cited per illness was 1.1. As noted in other studies (Quinlan and Quinlan 2007) this is not because most participants knew one kastom medicine per treatment; rather, most knew many treatments for some illnesses (i.e. for treating cuts: $M=2.05$, median=2) and very few for others (e.g. arthritis: $M=0.49$, median=0). Moreover, some plants were commonly used to treat a wide range of ailments: for example the shrub known as nahambis in Bislama (Euphorbiaceae) was cited by at least one participant in all ten freelists (and 48 times overall). 
Table 5.6: Descriptive statistics for medicinal plants cited in ten illness domains (for all participants: $n=$ 177)

\begin{tabular}{lccccc}
\hline Illness freelist & $\mathbf{H}^{\prime}$ & $\begin{array}{c}\text { Species } \\
\text { richness }\end{array}$ & $\begin{array}{c}\text { Citation } \\
\text { abundance }\end{array}$ & Evenness & $\begin{array}{c}\text { Mean abundance per } \\
\text { participant }\end{array}$ \\
\hline Headache & 4.27 & 95 & 201 & 0.94 & 1.14 \\
Cough & 3.76 & 77 & 284 & 0.87 & 1.60 \\
Earache & 3.23 & 50 & 175 & 0.83 & 0.99 \\
Diarrhoea & 3.69 & 70 & 226 & 0.87 & 1.28 \\
Toothache & 3.59 & 57 & 230 & 0.89 & 1.30 \\
Cuts and & & & & & 2.05 \\
abrasions & 2.91 & 59 & 362 & 0.71 & 0.46 \\
Arthritis & 3.88 & 57 & 82 & 0.96 & 0.93 \\
Malaria & 2.92 & 46 & 164 & 0.76 & 1.18 \\
Hepatitis & 3.51 & 69 & 209 & 0.83 & 0.51 \\
Conjunctivitis & 2.72 & 29 & 90 & 0.81 & $\mathbf{1 1 . 0 9}$ \\
Total & $\mathbf{4 . 6 5}$ & $\mathbf{2 6 2}$ & $\mathbf{1 9 7 4}$ & $\mathbf{0 . 8 3}$ & \\
\hline
\end{tabular}

Bivariate spearman's rank correlations were conducted between medicinal plant dependent variables (citation richness, abundance, and diversity) and the predictor variables (Table 5.7). Some of the hypotheses set out in Table 5.1 were supported by significant bivariate correlations: age correlated positively with all three dependent variables, as did linguistic preference and residence at Dixon Reef. Others were not supported: where no relationship was predicted, all three dependent variables displayed significant negative correlations with female gender, indicating that men consistently scored higher. Other predictor variables, such as church attendance, education attainment, birth location, and residence at Tisvel or Lawa did not show a significant correlation with any of the medicinal plant dependent variables. 
Table 5.7: Bivariate correlations of dependent variables with predictor variables

\begin{tabular}{|c|c|c|c|c|c|c|c|c|c|c|c|}
\hline & Age & Gender & $\begin{array}{l}\text { Birth } \\
\text { locality }\end{array}$ & $\begin{array}{l}\text { Educational } \\
\text { attainment }\end{array}$ & $\begin{array}{l}\text { Church } \\
\text { attendance }\end{array}$ & $\begin{array}{l}\text { Linguistic } \\
\text { preference }\end{array}$ & $\begin{array}{l}\text { Market } \\
\text { visits }\end{array}$ & $\begin{array}{l}\text { Commercial } \\
\text { occupation }\end{array}$ & Tisvel & $\begin{array}{c}\text { Dixon } \\
\text { Reef }\end{array}$ & Lawa \\
\hline 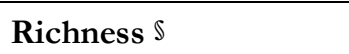 & $.150^{*}$ & $.261^{* *}$ & -.048 & -.046 & .014 & $.173^{*}$ & -.122 & -.111 & -.099 & $.149^{*}$ & .080 \\
\hline Abundance $\mathbb{}$ & $.153^{*}$ & $.268^{* *}$ & -.059 & -.052 & .005 & $.186^{*}$ & -.114 & -.107 & -.114 & .127 & .100 \\
\hline Shannon-Weiner & $.152^{*}$ & $.258^{* *}$ & -.048 & -.081 & .128 & $.166^{*}$ & $-.253^{* *}$ & -.130 & -.090 & $.184^{*}$ & .054 \\
\hline Abundance (Fish) $\mathbb{}$ & $-.326^{* *}$ & $.207^{* *}$ & .024 & $.359^{* *}$ & $-.227^{* *}$ & -.103 & $.269^{* *}$ & .110 & $.194^{*}$ & .147 & -.032 \\
\hline Ecological knowledge & $.260^{* *}$ & $.369^{* *}$ & $-.286^{* *}$ & -.087 & -.028 & $.365^{* *}$ & -.087 & -.037 & .006 & .005 & .071 \\
\hline Proficiency score & $.209^{* *}$ & $.387^{* *}$ & -.028 & .024 & .152 & $.195^{*}$ & -.050 & -.106 & -.151 & $.173^{*}$ & .144 \\
\hline
\end{tabular}

$*=$ correlation significant at the 0.05 level; $* *=$ correlation significant at the 0.01 level

NB: Variables marked $\mathbb{\$}$ report Spearman's rank coefficients; others report Pearson's correlations. Variables above dashed line refer to medicinal plant citations 
Some key trends warrant further comment. There were moderately strong associations with gender, with men being able to name significantly higher richness of plants than women across all freelists (significant using Mann-Whitney non-parametric ranking: $\mathrm{U}=2682.5, \mathrm{p}<.001$ ). This relationship was not observed within all communities, however, and proved to be significant only in the more isolated communities of Lawa and Dixon Reef (respectively, $\mathrm{U}=66.5, \mathrm{p}<.001$; and $\mathrm{U}=104.5, \mathrm{p}=.05$ ). At Unmet median richness of species cited was marginally higher for female participants; however gender differences were not significant at Unmet or Tisvel (Figure 5.2).

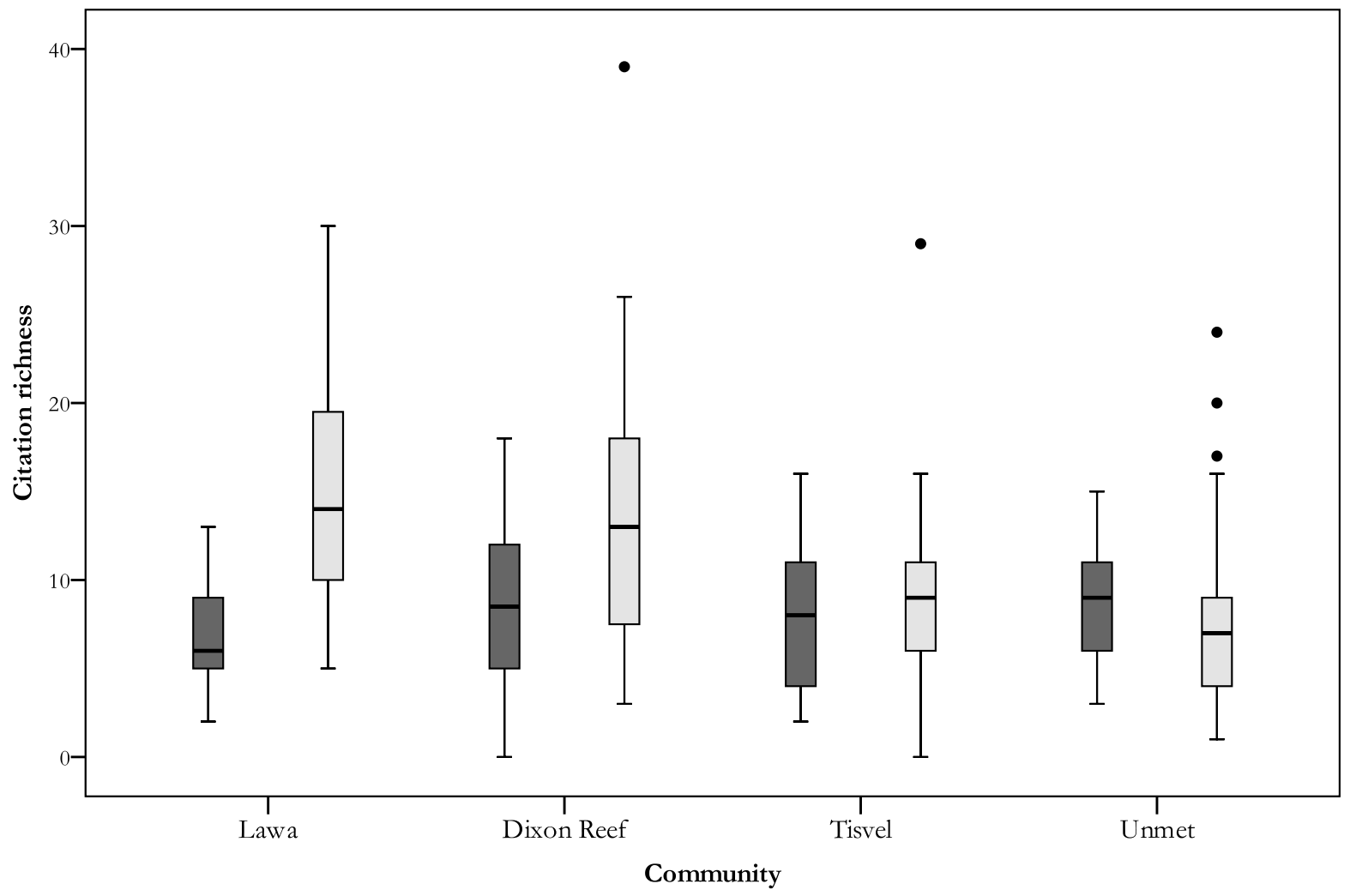

Figure 5.2: Richness of cited medicinal plants across ten freelists, per community and gender (dark boxes indicate female participants, light boxes indicate male participants). Significant difference between male and female at Lawa, $\mathrm{p}<.001$; significant difference between male and female at Dixon Reef, $\mathrm{p}<.05$

In total, richness and diversity of plant citations was highest at Dixon Reef $\left(M_{\text {Richness }}=12.00 ; M_{\mathrm{H}^{\prime}}=\right.$ 2.31), and lowest at Unmet $\left(M_{\text {Richness }}=8.51 ; M_{\mathrm{H}}=1.96\right)$. Kruskal-Wallis non-parametric ranking reveals that scores for both variables are highest at Dixon Reef and Lawa, and that this difference was marginally significant (for both, $\mathrm{p}=.05$ ). However, post-hoc testing using Mann-Whitney nonparametric ranking and a Bonferroni correction (thus reporting significance at the 0.0167 level) revealed that these differences were not significant. 
There was also evidence of demographic correlations with the kinds of plants that were cited by informants, if species were roughly coded as 'cultivated' or 'wild'. Percentage of cultivated plants cited displayed a weak yet significant negative bivariate correlations with age $\left(r_{s}=-0.153, p<.05\right)$, and was also significantly associated with preferential use of Bislama $\left(\mathrm{r}_{\mathrm{s}}=-0.235, \mathrm{p}<.001\right)$ and with female gender $\left(r_{s}=-0.262, p<.001\right)$. Note that both variables are binary and coded as noted in Table 5.1; therefore, negative associations indicate relationships as shown. If age is split into six roughly equal cohorts, there is a significant cohort effect visible (Figure 5.3): whilst there are no significant differences between the first five cohorts, the $70+$ cohort $(n=11)$ cite a significantly lower percentage of cultivated plants, and thus a higher percentage of wild plants (Mann-Whitney non-parametric ranking, $U=488.5, \mathrm{p}<.05)$.

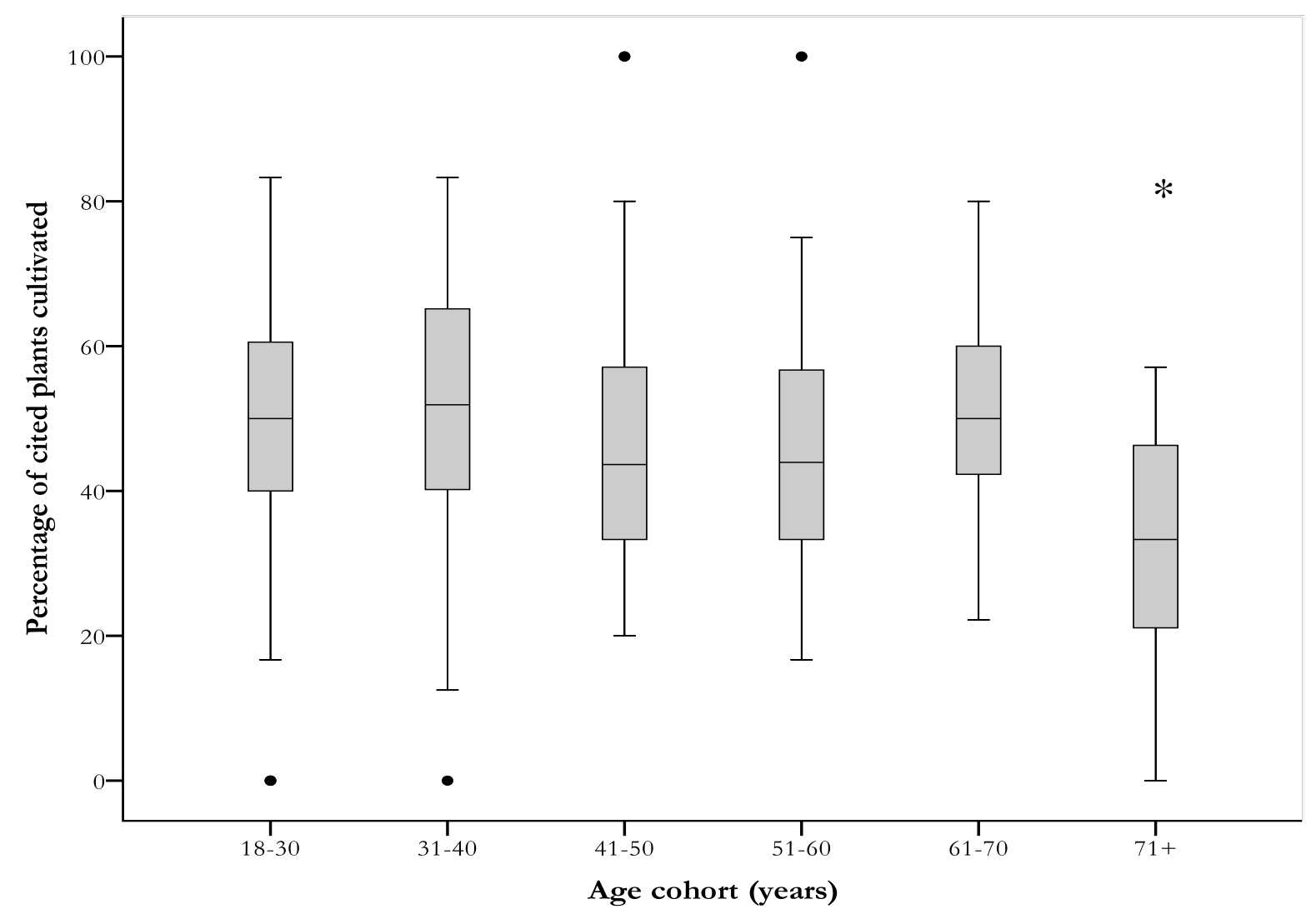

Figure 5.3: Mean percentage of cited plants cultivated, per age cohort. $*$ denotes significantly different to other age cohorts, $\mathrm{p}<0.05$. denotes outlier.

\subsubsection{Modelling variation in citation richness}

I ran the AIC model ranking procedure on the models outlined in Table 5.5. Results are displayed in Table 5.8. 
Table 5.8: Model ranking for LogRichness of citations from ten illness freelists

\begin{tabular}{|c|c|c|c|c|c|c|}
\hline Hypothesis & Variables included in model & RSS & $\mathrm{AIC}_{\mathrm{c}}$ & $\Delta \mathrm{AIC}_{\mathrm{c}}$ & $\mathrm{AIC}_{\mathrm{c} \cdot \mathrm{w}}$ & $\mathrm{r}^{2}$ \\
\hline 8 & $\begin{array}{l}\text { Age, gender, education, linguistic preference, } \\
\text { market visitation }\end{array}$ & 10.62 & -471.85 & 0 & 0.93 & 0.174 \\
\hline 1 & All variables & 10.13 & -466.47 & 5.53 & 0.06 & 0.212 \\
\hline 5 & Age, gender & 11.77 & -460.45 & 11.55 & 0.00 & 0.084 \\
\hline 11 & $\begin{array}{l}\text { Gender and community dummy variables } \\
\text { only }\end{array}$ & 11.49 & -460.34 & 11.66 & 0.00 & 0.106 \\
\hline 7 & Age, gender, education, linguistic preference & 11.56 & -459.37 & 12.63 & 0.00 & 0.101 \\
\hline 2 & All demographic variables & 11.77 & -458.34 & 13.66 & 0.00 & 0.084 \\
\hline 6 & Age, gender, education & 11.77 & -458.33 & 13.67 & 0.00 & 0.084 \\
\hline 3 & All modernisation variables & 11.49 & -458.18 & 13.82 & 0.00 & 0.106 \\
\hline 9 & $\begin{array}{l}\text { All community dummy variables, market } \\
\text { visitation }\end{array}$ & 11.66 & -457.79 & 14.21 & 0.00 & 0.093 \\
\hline 10 & $\begin{array}{l}\text { All modernisation \& community dummy } \\
\text { variables }\end{array}$ & 11.23 & -455.49 & 16.51 & 0.00 & 0.126 \\
\hline 4 & All community dummy variables & 12.26 & -451.16 & 20.84 & 0.00 & 0.046 \\
\hline
\end{tabular}

The right hand columns indicate important information about each model. RSS (residual sum of squares) is a measure of the variation in the data that cannot be explain by the model, and is used to calculate the $\mathrm{AIC}_{\mathrm{c}}$ value. $\triangle \mathrm{AIC}_{\mathrm{c}}$ denotes the change in $\mathrm{AIC}_{\mathrm{c}}$ values between models; a rule of thumb is that all models with a $\triangle \mathrm{AIC}_{\mathrm{c}}<2$ should be included in the best model subset (Burnham and Anderson 1998). AIC $\mathrm{c} \cdot \mathrm{w}$ is the Akaike weight, and indicates the relative likelihood that the model is the most correct for the data. Finally, the $\mathrm{r}^{2}$ value indicates the amount of variance in the data that each model can explain. In the social sciences, an $\mathrm{r}^{2}$ value of 0.20 (indicating that the model can explain $20 \%$ of the variance in the data) is considered to be very promising; a value of 0.40 is accurate; and an $\mathrm{r}^{2}$ over 0.60 is 'cause for celebration' (Quinlan and Quinlan 2007: p 178).

Table 5.8 indicates that for LogRichness of medicinal plants cited, there is only one model with $\Delta \mathrm{AIC}_{\mathrm{c}}<2$ and that this includes the variables of age, gender, educational attainment, linguistic preference, and market visitation. The $\mathrm{AIC}_{\mathrm{c}} \mathrm{w}$ value indicates that this model is easily the most likely, despite the fact that the next model (which includes all predictor variables) has a higher $\mathrm{r}^{2}$ value. The full multiple regression output is outlined in Table 5.9 below.

Table 5.9: Multiple regression model for LogRichness under Model Eight

\begin{tabular}{lccc}
\hline & B & SE B & B \\
\hline (Constant) & 0.68 & 0.10 & \\
Age & 0.00 & 0.00 & 0.11 \\
Gender (0=Female, 1=Male) & 0.16 & 0.04 & $0.29^{* *}$ \\
Educational attainment & 0.01 & 0.01 & 0.08 \\
Linguistic preference (0=Bislama, & 0.08 & 0.06 & $0.10^{*}$ \\
1=Vernacular language) & 0.00 & 0.00 & $-0.28^{* *}$ \\
Annual visits to market & & & \\
\hline Dependent variable: LogRichness & & \\
$r^{2}=0.174, n=176 . * *$
\end{tabular}


Of the five predictor variables in this model, gender, linguistic preference and annual market visitation have a significant influence. When converted from LogRichness to Richness, males cite 1.97 more species of medicinal plant than women; participants who preferentially use vernacular languages over Bislama cite 1.11 more medicinal species; and that for every increase in annual visit to market, participants cited 1.32 fewer medicinal plants. Both age and educational attainment showed a positive relationship with the dependent variable, however neither was significant.

\subsubsection{Modelling variation in individual-level Shannon index score}

Individual-level Shannon index scores were normally distributed. Once again, standard diagnostic plots revealed no issues that would influence results of multiple regression modelling. AIC model ranking was run as above (Table 5.10).

Table 5.10: AIC model ranking for Individual-level Shannon diversity index score

\begin{tabular}{clrrrrr}
\hline Hypothesis & Variables included & $\mathrm{RSS}$ & $\mathrm{AIC}_{\mathrm{c}}$ & $\Delta \mathrm{AIC}_{\mathrm{c}}$ & $\mathrm{AIC}_{\mathrm{c} . \mathrm{w}}$ & $\mathrm{R}^{2}$ \\
\hline & Age, gender, education, linguistic preference, & & & & & \\
8 & market visitation & 53.77 & -189.66 & 0.00 & 0.91 & 0.172 \\
1 & All variables & 51.14 & -184.78 & 4.88 & 0.08 & 0.212 \\
3 & All modernisation variables & 57.21 & -178.88 & 10.78 & 0.00 & 0.119 \\
& Gender and community dummy variables & & & & & \\
11 & only & 58.04 & -178.54 & 11.12 & 0.00 & 0.106 \\
5 & Age, gender & 59.60 & -178.20 & 11.46 & 0.00 & 0.082 \\
7 & Age, gender, education, linguistic preference & 58.55 & -177.03 & 12.63 & 0.00 & 0.098 \\
9 & All community dummy variables, market & & & & & \\
2 & visitation & 58.67 & -176.67 & 12.99 & 0.00 & 0.096 \\
6 & All demographic variables & 59.59 & -176.09 & 13.57 & 0.00 & 0.082 \\
& Age, gender, education & 59.59 & -176.08 & 13.58 & 0.00 & 0.082 \\
10 & All modernisation \& community dummy & & & & & \\
4 & variables & 56.47 & -174.46 & 15.20 & 0.00 & 0.13 \\
& All community dummy variables & 61.75 & -169.90 & 19.76 & 0.00 & 0.049 \\
\hline
\end{tabular}

Unsurprisingly, given the high individual-level association between richness and abundance, AIC analysis for individual Shannon score was very similar to that for LogRichness. Only one model, containing the same predictor variables as above, had a delta AIC value under 2 and was subsequently considered to be the 'best' model. The full multiple regression for this analysis is presented in Table 5.11 . 
Table 5.11: Multiple regression model for individual-level Shannon score under Model Eight

\begin{tabular}{lccc}
\hline & B & SE B & $\beta$ \\
\hline (Constant) & 1.57 & 0.23 & \\
Age & 0.00 & 0.00 & 0.10 \\
Gender $(0=$ Female, $1=$ Male) & 0.35 & 0.09 & $0.29^{* *}$ \\
Educational attainment & 0.02 & 0.02 & 0.08 \\
Linguistic preference $(0=$ Bislama, & 0.18 & 0.13 & $0.10^{*}$ \\
1=Vernacular language) & & & \\
Annual visits to market & -0.01 & 0.00 & $-0.28^{* *}$ \\
\hline
\end{tabular}

Dependent variable: Individual-level Shannon score

$r^{2}=0.172, n=174 . * *=p<0.001 ; *=p<0.05$

Once again, this model is very closely related to Table 5.9 above, and notably the beta values (which are standardised, and thus reflect the standard deviation of the model) are nearly exactly the same. When all other variables are controlled, males typically have an H' score 0.35 higher than females; those who favour vernacular languages over Bislama score 0.18 higher; and each annual visit to market associated with a drop of 0.01 in an individual's H' score. Once again, age and educational attainment show a positive association with the dependent variable, however neither is significant.

\subsubsection{Group level Shannon index scores}

Because the diversity index is based on $\mathrm{p}_{\mathrm{i}}$, or the proportion of total citations, they are also useful for comparing between categories, as population-level differences in citation richness may be evident where individual-level variation is not. I utilised the predictor variables in Model Eight as this was the best model for the two preceding multiple regressions, and included community (

Table 5.12). For comparative purposes I created categorical variables for age (three cohorts: 18-30, 31-60, and 60+); market visitation (based on sample size, 0 market visits; 1-2 market visits; 3-4 market visits; and $5+$ market visits per year); and educational attainment (based on sample size 0-5 years of educational attainment; attainment to year 6; and over year 6). 
Table 5.12: Comparison between categories based on Model Eight

\begin{tabular}{|c|c|c|c|c|c|c|}
\hline & & $\begin{array}{l}\text { Number of } \\
\text { informants }\end{array}$ & Abundance & Richness & $\mathbf{H}^{\prime}$ & Evenness \\
\hline \multirow{4}{*}{ 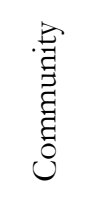 } & Lawa & 49 & 613 & 129 & 4.13 & 0.85 \\
\hline & Dixon & 38 & 503 & 128 & 4.36 & 0.90 \\
\hline & Tisvel & 43 & 414 & 99 & 3.98 & 0.87 \\
\hline & Unmet & 47 & 444 & 100 & 3.98 & 0.86 \\
\hline \multirow{2}{*}{$\begin{array}{l}\ddot{J} \\
\vec{U} \\
\overrightarrow{0}\end{array}$} & Male & 94 & 1254 & 238 & 4.70 & 0.86 \\
\hline & Female & 83 & 720 & 145 & 4.27 & 0.86 \\
\hline \multirow{3}{*}{$\begin{array}{l}0 \\
0 \\
0 \\
0 \\
0 \\
0 \\
0\end{array}$} & $18-30$ & 59 & 560 & 148 & 4.3 & 0.86 \\
\hline & $31-60$ & 90 & 1049 & 186 & 4.48 & 0.86 \\
\hline & $61+$ & 28 & 356 & 145 & 4.62 & 0.93 \\
\hline \multirow{2}{*}{ 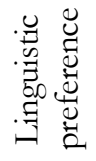 } & Vernacular & 154 & 1781 & 254 & 4.67 & 0.84 \\
\hline & Bislama & 23 & 193 & 74 & 3.80 & 0.88 \\
\hline \multirow{4}{*}{ 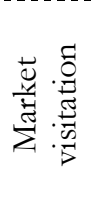 } & 0 & 41 & 451 & 153 & 4.46 & 0.89 \\
\hline & $1-2$ & 47 & 634 & 170 & 4.56 & 0.89 \\
\hline & $3-4$ & 47 & 477 & 129 & 4.24 & 0.87 \\
\hline & $5+$ & 42 & 403 & 117 & 4.20 & 0.88 \\
\hline \multirow{3}{*}{ 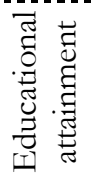 } & $0-5$ & 58 & 646 & 189 & 4.68 & 0.89 \\
\hline & 6 & 75 & 912 & 189 & 4.51 & 0.86 \\
\hline & $7+$ & 44 & 407 & 111 & 4.10 & 0.87 \\
\hline Total & & 177 & 1974 & 263 & 4.65 & 0.83 \\
\hline
\end{tabular}

NB: Selected t-tests as per Magurran (1988); $N=$ abundance of citations, $S=$ richness of species cited: Community: Lawa vs. Unmet $t=18.9, p<.001, d f=973.29$; Dixon vs. Unmet $t=48.9, p<$ $.001, d f=873.98$; Tisvel vs. Unmet $t=0.732, p>0.50, d f=856.14$

Gender: $t=82.96, p<.001, d f=1548.37$

Age cohort: $18-30$ vs. $60+t=104.81, p<.002, d f=835.48 ; 18-30 v s .31-60 t=99.48, p$ $<.001, d f=832.38 ; 31-60 v s .60+t=26.82, p<.001, d f=903.58$

Linguistic preference: $t=84.81, p<.001, d f=264.85$

Market Visitation: 0 vs. $5+t=28.29, p<.001, d f=841.21 ; 1-2 v s .5+t=45.03, p<.001$, $d f=843.07 ; 3-5$ vs. $5+t=3.49, p<.001, d f=863.49$

Educational attainment: $\underline{\text { Yr 1-5 vs. } y r 6} t=31.08, p<.001, d f=1490.49 ; y r 1-5$ vs. $y r$ $\underline{6+} t=74.06, p<.001, d f=835.24 ; y r 6 v s . y r .6+t=53.70, P<.001, d f=847.61$

Categorical comparison of group-level Shannon index scores reveals some interesting patterns. By community, the highest diversity of plants was cited at Lawa and Dixon Reef, whereas Tisvel and Unmet were not significantly different. Men cited a higher diversity of plants than women, though the evenness of both groups was the same. There were significant differences evident between age cohorts, indicating that the oldest age cohort as a group cites a higher diversity of plants than the younger two. Moreover, the evenness of citations of the oldest cohort was higher, indicating a high number of unique citations within the group, or a low overall level of homogeneity. The difference between this finding and the regression results above (showing that age is not a significant factor in 
individual-level richness of citations) is likely to be due to the fact that the relationship shown here is not linear. As such, it would not be evident in regression analyses, which assume linearity.

Significant differences were also evident between cohorts of market integration and education and between those who preferentially use Bislama against those who use vernacular languages, in line with the hypotheses suggested in Table 5.1.

\subsubsection{Rarefaction}

I drew standard Mao-Tao rarefaction curves to illustrate the comparisons above (Figure $5.4 \mathrm{a}-\mathrm{f}$ )). The $\mathrm{x}$-axes indicate number of informants per sample, and the y-axes are scaled by the expected species richness of the rarefied sample. Dotted lines indicate $95 \%$ confidence intervals for each curve, where groups do not overlap the differences between them are ostensibly significant (Williams et al. 2007). 
a)

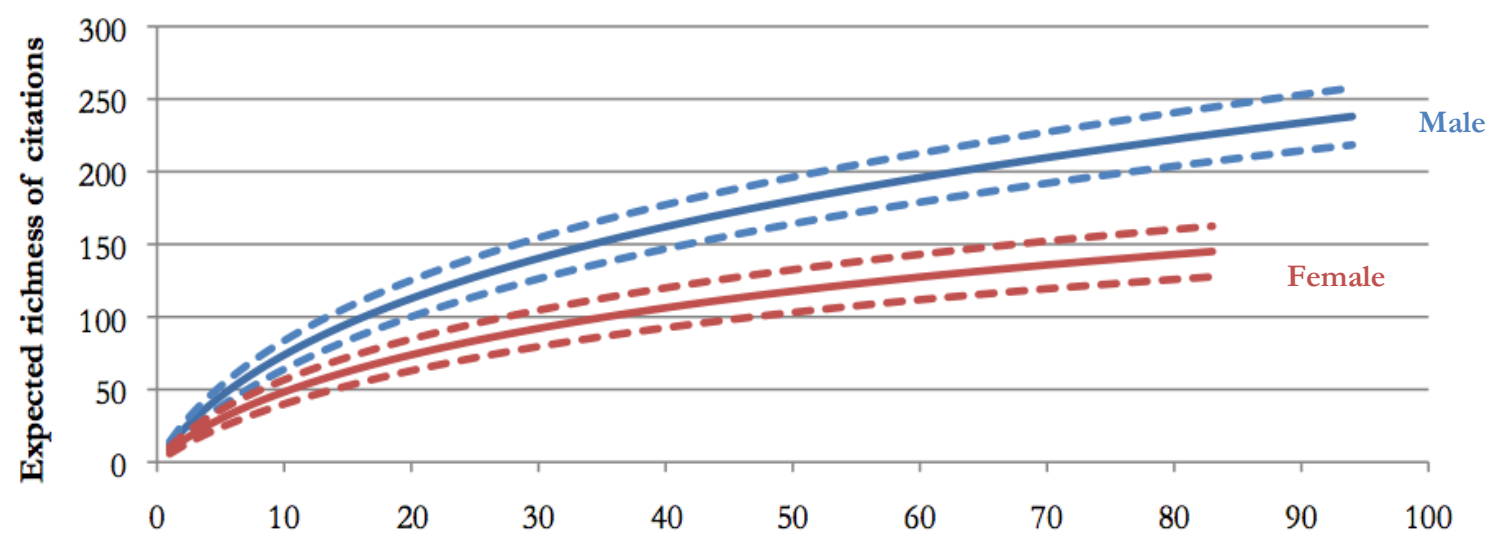

Number of informants

b)

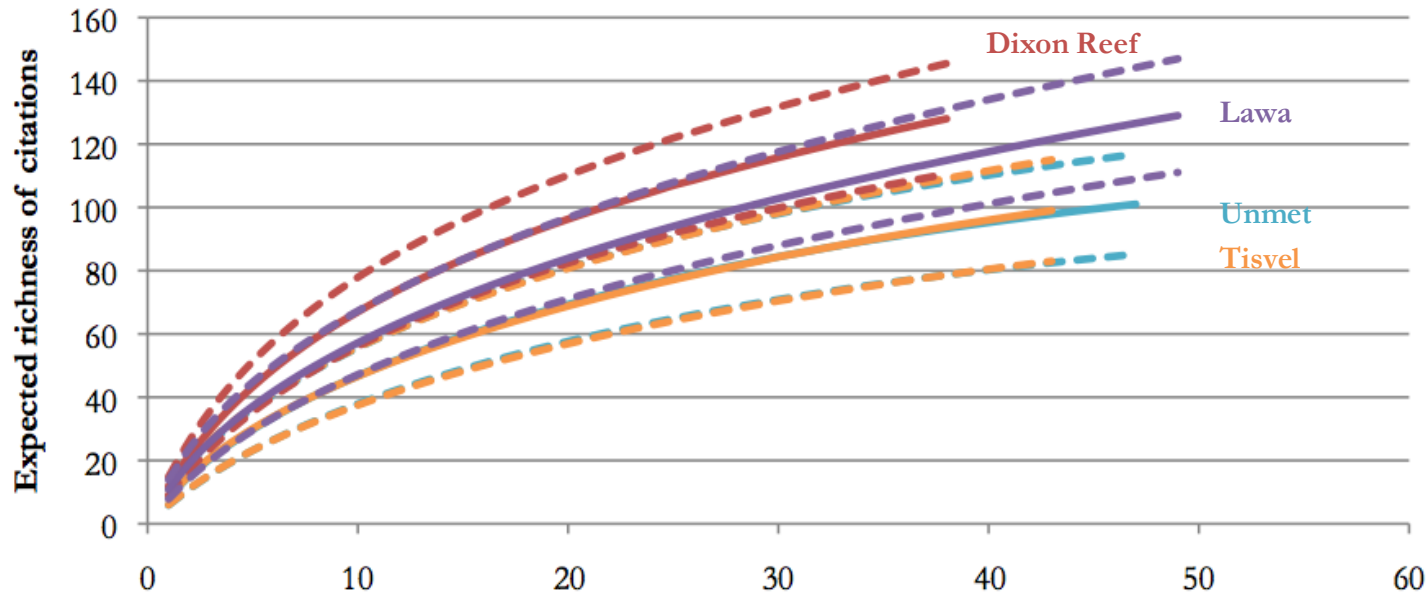

Number of informants

c)

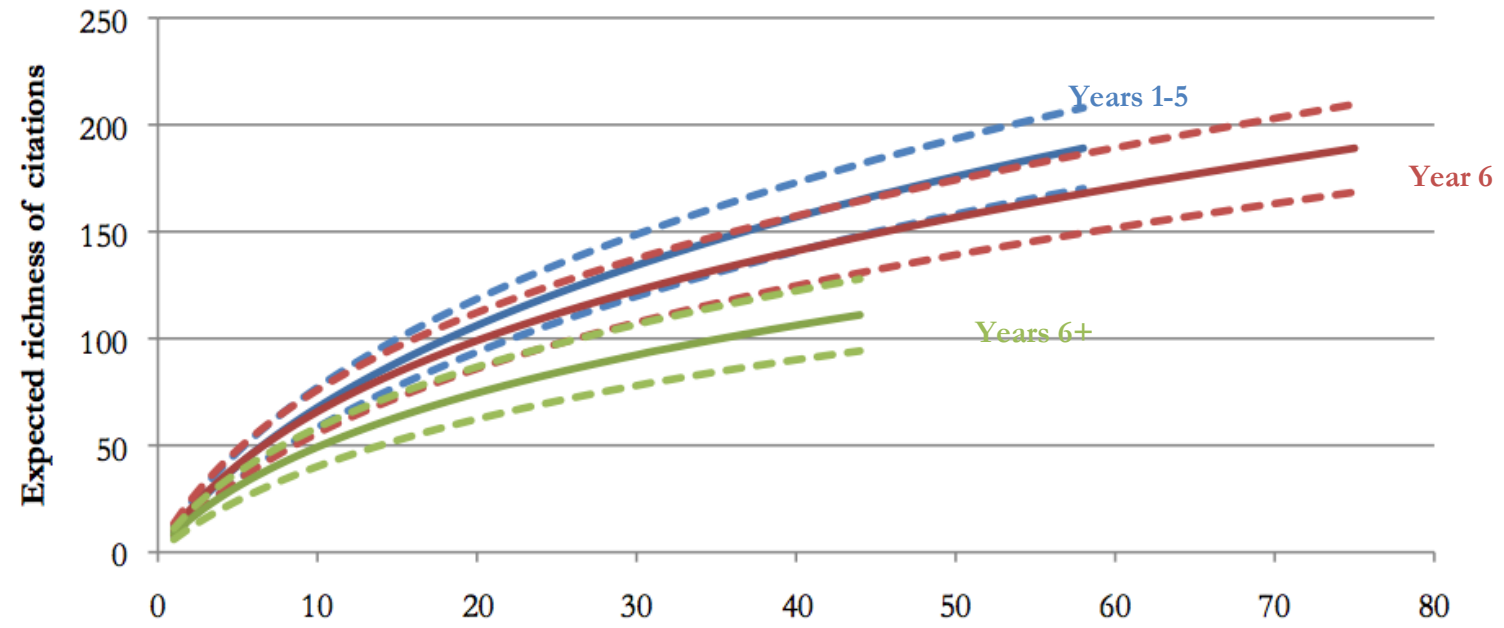

Number of informants 
d)

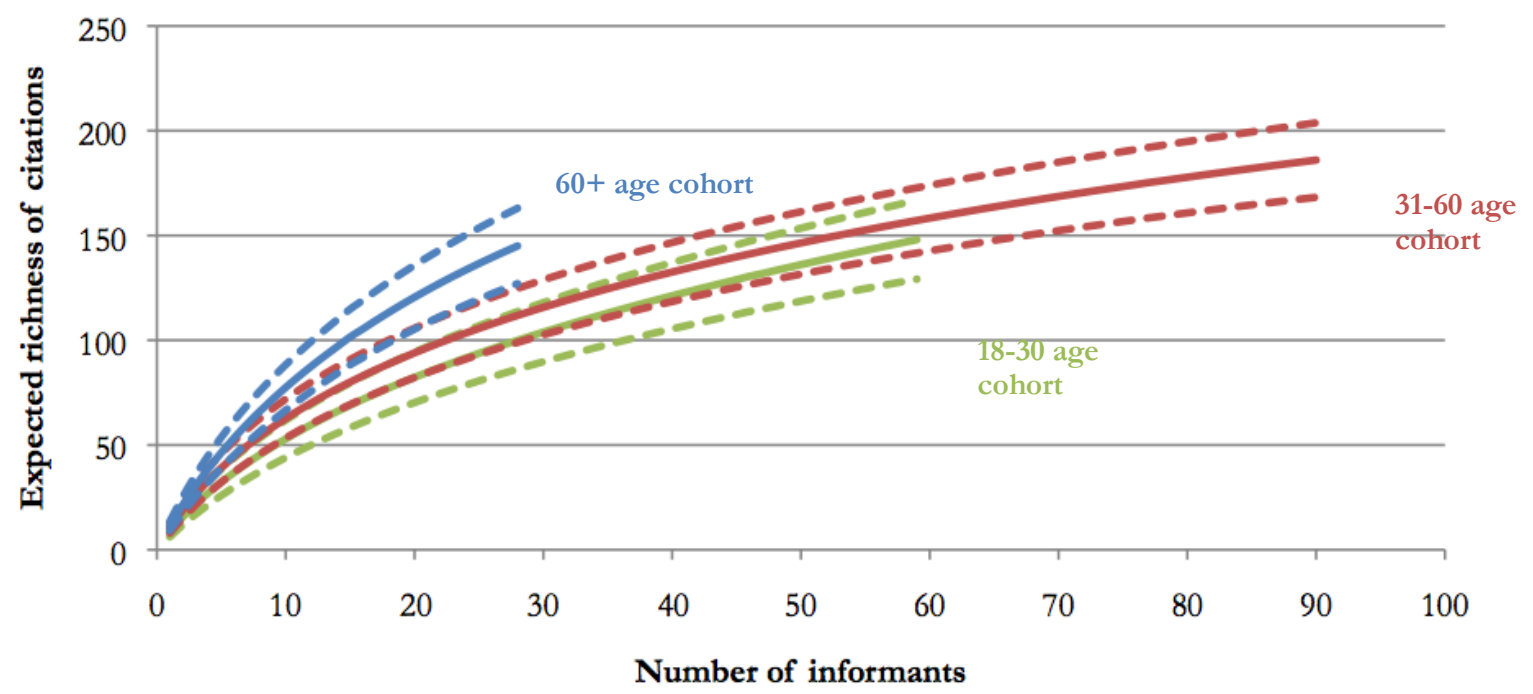

e)

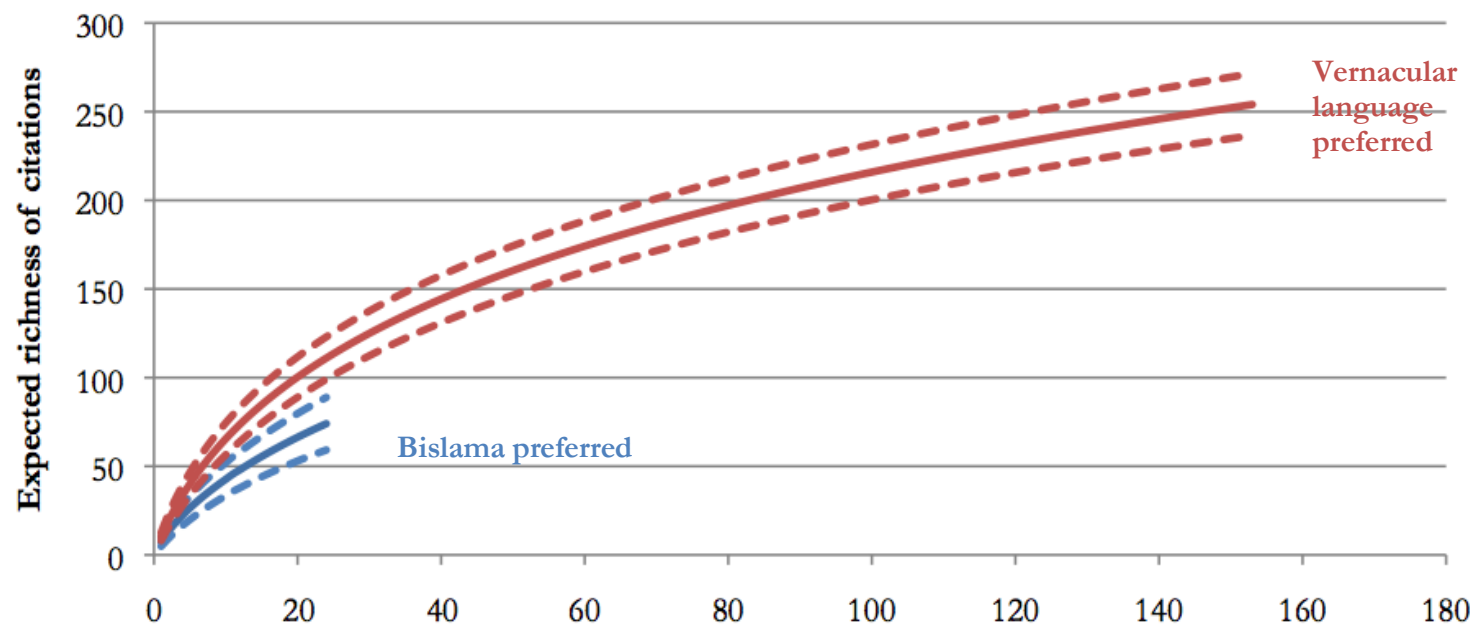

Number of informants

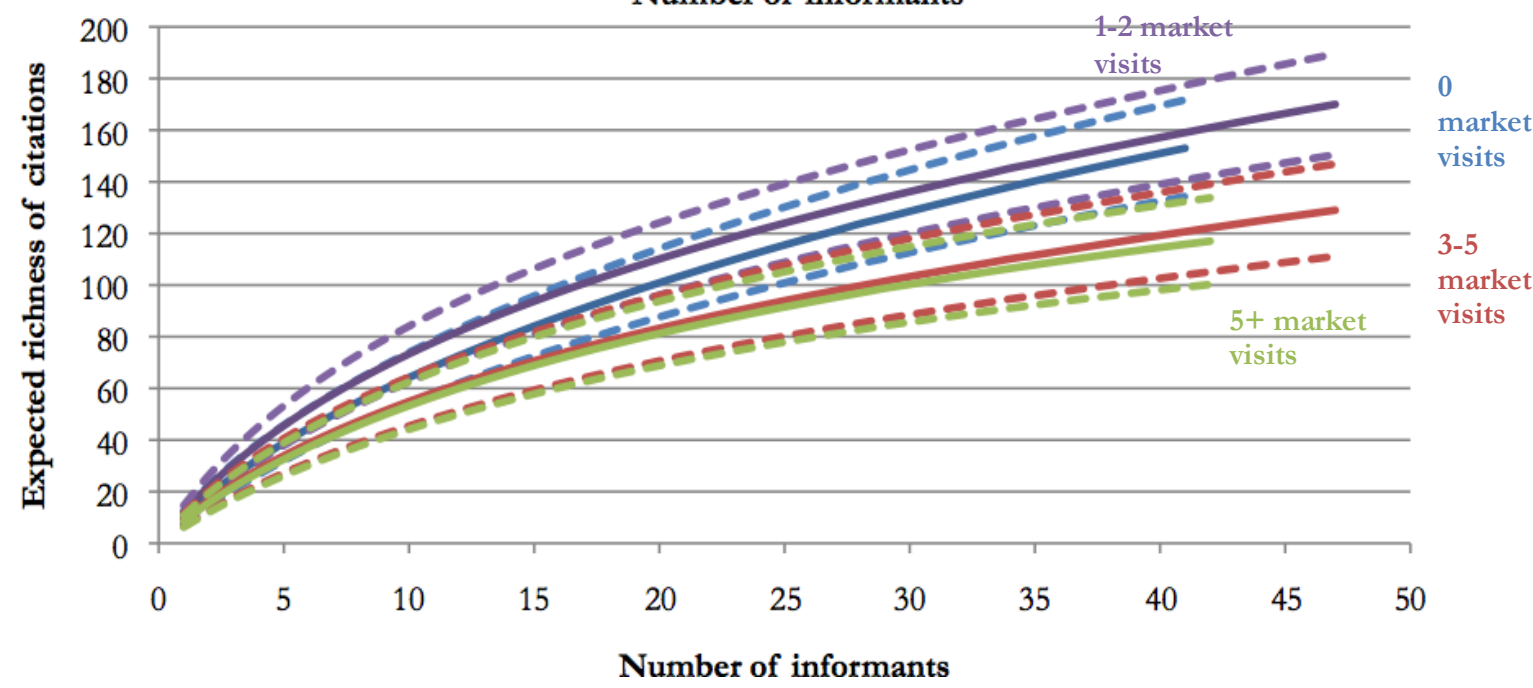

Figure 5.4: Mao-Tao rarefaction curves of expected richness vs. number of informants with variables under hypothesis eight: a) per gender; b) per community; c) per educational attainment category; d) per age cohort; e) per linguistic preference; f) per market visit 
Figure 5.4 a)-f) largely corroborate trends from the categorical comparisons indicated in Table 5.12. The clearest differences were evident between male and female informants (Figure 5.4 a)); between Dixon Reef and Unmet/Tisvel (Figure 5.4 b)); between those with 1-5 years education and those with over 6 years of formal education (Figure $5.4 \mathrm{c}$ )); between the oldest and youngest age cohorts (Figure $5.4 \mathrm{~d}$ )); between those who preferentially use vernacular language over Bislama (Figure 5.4e)); and between those who make between zero and two market visits per year over those who make three and over market visits a year (Figure $5.4 \mathrm{f}$ )). Where the 95\% confidence intervals do not overlap they are ostensibly significant. This criterion would appear to be a more stringent test than the t-tests in

Table 5.12, which found several additional significant categorical differences.

Figure 5.4 d) and e) also draw attention to the issue of sample size between the categorical predictions. The ability of a species accumulation curve to reach an asymptote is a good indicator that sampling has been sufficient (Williams et al. 2007). Whilst none of the curves fully reach asymptote, I observe that most are flattening. However, the trajectory of the curves for those who preferentially use Bislama, and for the $60+$ age cohort are not approaching asymptote, meaning that the number of species added to the total sample is still increasing. This means that these results must be viewed with caution, as I do not know if they would asymptote at a higher species richness than their categorical comparisons. However, the 95\% confidence intervals and t-tests indicate that the samples are significantly different, which means that increased sampling would be likely to only increase the differences observed.

\subsubsection{Richness estimators}

Finally, I plotted the total richness from the ten medicinal plant freelists against two commonly used richness estimators, as a means of gauging the completeness of the total sample (Figure 5.5). The sample appears to be close to crossing the asymptotic MMMean estimator, however the Jack1 nonparametric estimator indicates does not appear to be close to asymptote. Williams et al. (2007) find that the Jack1 estimator is the most reliable. This indicates that the body of medicinal plant knowledge is potentially much greater than that sampled by the ten freelists, and confirms the need for further sampling. 


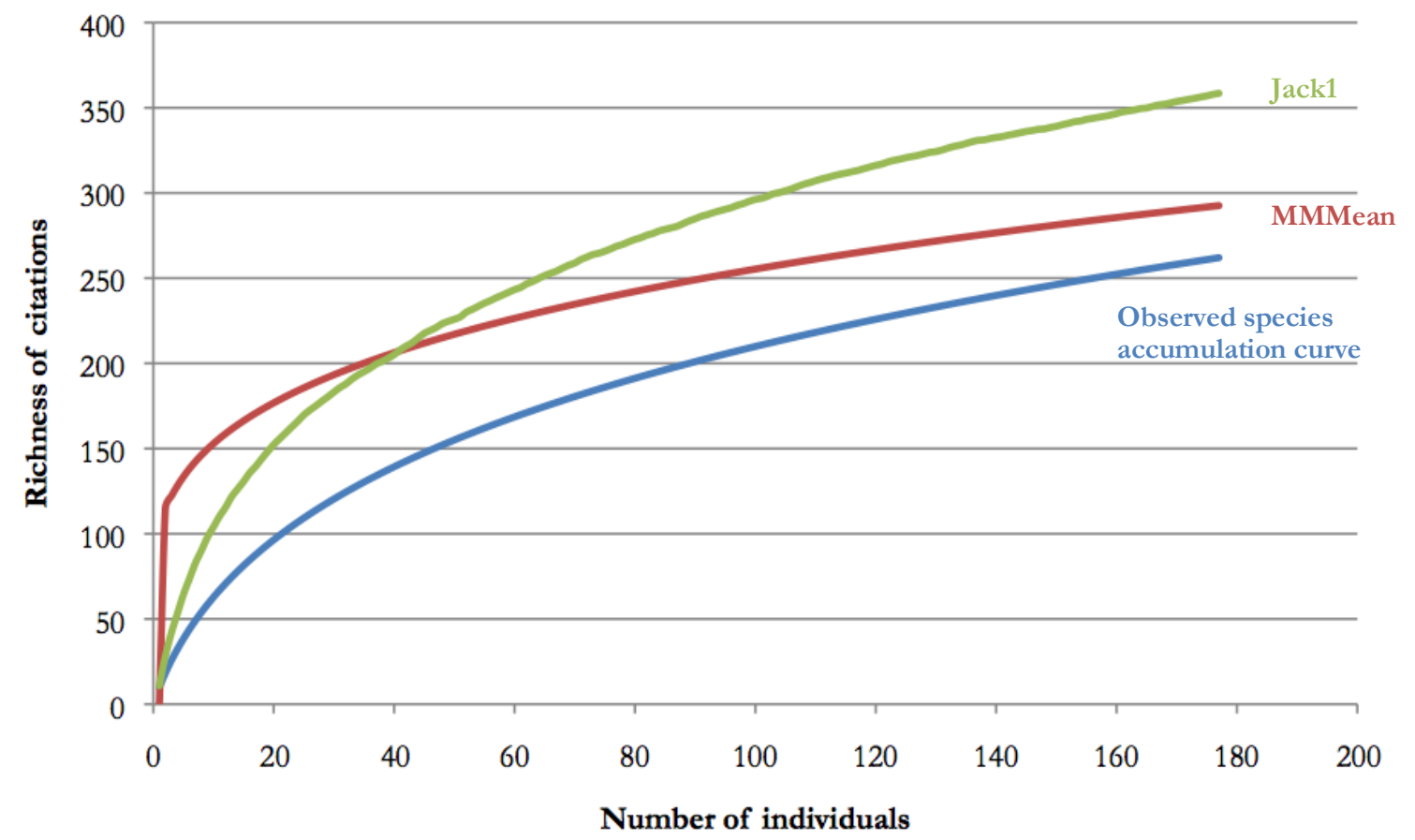

Figure 5.5: Jack1 and MMMeans richness estimators against Mao-Tao species accumulation curve

\subsubsection{Supplementary TEK measures}

I took three further measures of TEK to supplement the medicinal plant data. I analyse the variation in these measures below.

\subsubsection{Knowledge of reef fish}

Participants listed an average 11.11 fish per freelist, with a median of 10. Only one participant was not able to cite any plants, and the highest number of fish cited was 42. Citations were not identified using scientific nomenclature; as such, I discuss individual level citation abundance only.

Individual abundance of citations of reef fish upheld two of the hypotheses from the introduction: there was a significant negative correlation with church attendance $(\mathrm{r}=-0.227, \mathrm{p}<.001)$, and a positive association with residence at Tisvel $(\mathrm{r}=0.194, \mathrm{p}<.001$; full results in Table 5.7). Other results, however, contrasted with the direction suggested in the hypotheses: reef fish citation abundance was strongly associated with male gender (coded as female $=0$, male $=1 ; \mathrm{r}=0.207, \mathrm{p}$ $<.001)$; positively associated with educational attainment $(\mathrm{r}=0.359, \mathrm{p}<.001)$ and annual market visitation $(\mathrm{r}=0.269, \mathrm{p}<.001)$; and negatively associated with age $(\mathrm{r}=-0.326, \mathrm{p}<.001)$. Significant correlations with categorical variables (gender and residence at Tisvel) were significant using MannWhitney non-parametric ranking (respectively $U=2782, \mathrm{p}<.01 ; U=2006.5, \mathrm{p}<.05$ ). 
Differences between communities were shown to be significant through Kruskal-Wallis nonparametric ranking $(\mathrm{p}<.05)$, however post-hoc testing (Mann-Whitney non-parametric ranking with a Bonniferoni correction to observe significance at the 0.0167 level) only the difference between Tisvel (median citation abundance $=12$ ) and Unmet (median citation abundance $=7$ ) was significant. As with number of medicinal plants cited above, differences between male and female citation abundance were more pronounced in Lawa and Dixon Reef than in Tisvel and Unmet, however this was only statistically significant at Lawa $(\mathrm{U}=80.5, \mathrm{p}<.001$; Figure 5.6).

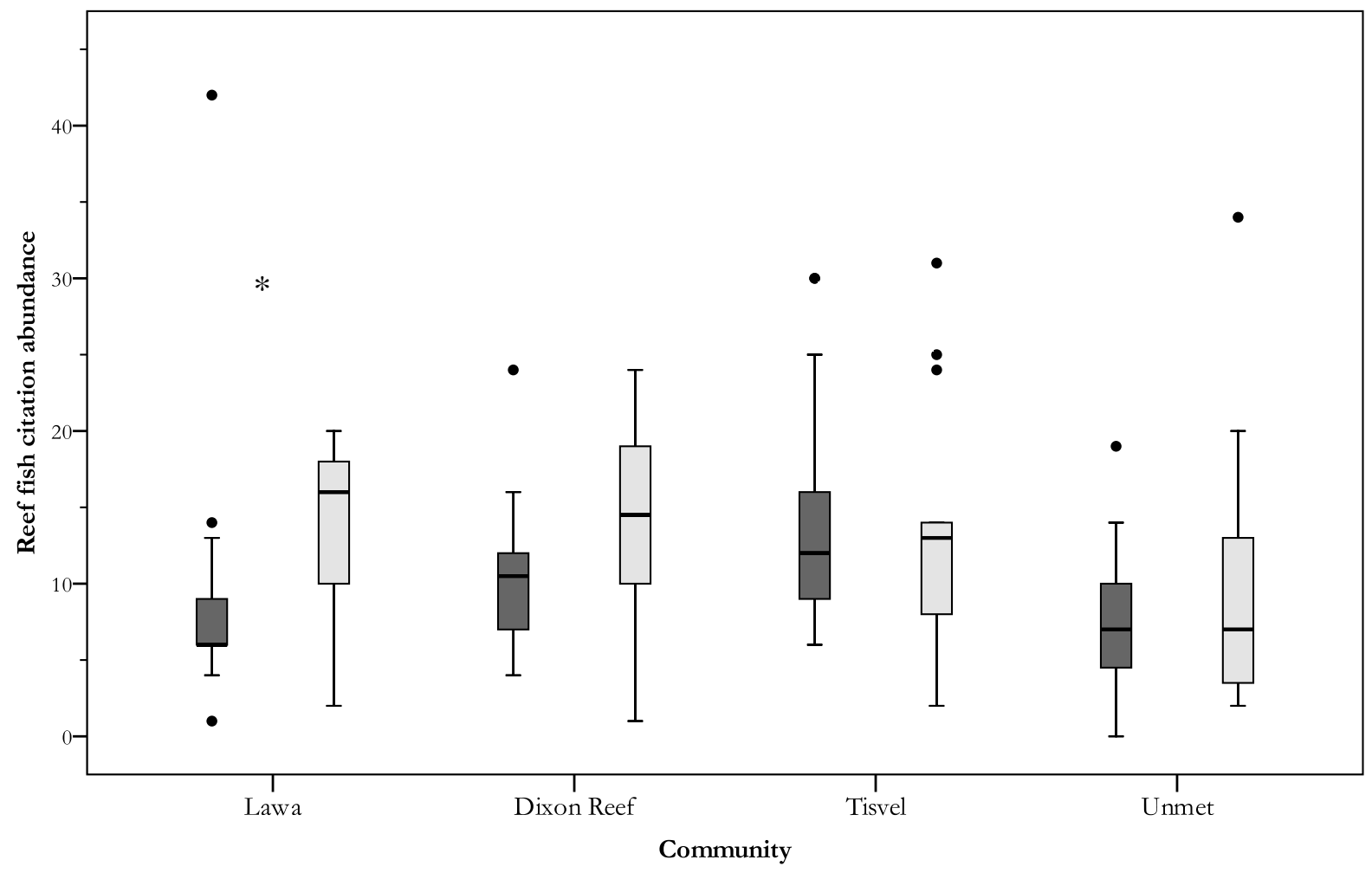

Figure 5.6: Abundance of reef fish citations per community, split via gender (dark boxes indicate female, light boxes indicate male; $*=$ difference between male and female participants at $\mathrm{p}<.001$ ).

\subsection{Model selection and multiple regression}

Citation abundance for reef fish was square root transformed to correct for non-normality. Standard diagnostic plots did not reveal any issues, so I proceeded with AIC model-ranking procedure as described above (Table 5.13). 
Table 5.13: AIC model ranking for square root reef fish citation abundance

\begin{tabular}{|c|c|c|c|c|c|c|}
\hline Hypothesis & Variables included & RSS & $\mathrm{AIC}_{\mathrm{c}}$ & $\Delta \mathrm{AIC}_{\mathrm{c}}$ & $\mathrm{AIC}_{\mathrm{c} \cdot \mathrm{w}}$ & $\mathbf{r}^{2}$ \\
\hline 1 & All variables & 123.76 & -29.94 & 0.00 & 0.80 & 0.308 \\
\hline 10 & $\begin{array}{l}\text { All modernisation \& community dummy } \\
\text { variables }\end{array}$ & 132.63 & -25.04 & 4.90 & 0.07 & 0.259 \\
\hline 6 & Age, gender, education & 141.46 & -25.04 & 4.90 & 0.07 & 0.21 \\
\hline 8 & $\begin{array}{l}\text { Age, gender, education, linguistic } \\
\text { preference, market visitation }\end{array}$ & 139.41 & -23.21 & 6.73 & 0.03 & 0.221 \\
\hline 7 & $\begin{array}{l}\text { Age, gender, education, linguistic } \\
\text { preference }\end{array}$ & 141.20 & -23.21 & 6.73 & 0.03 & 0.211 \\
\hline 5 & Age, gender & 151.98 & -14.90 & 15.04 & 0.00 & 0.151 \\
\hline 2 & All demographic variables & 151.79 & -12.98 & 16.96 & 0.00 & 0.152 \\
\hline 3 & All modernisation variables & 148.63 & -12.27 & 17.68 & 0.00 & 0.17 \\
\hline 11 & $\begin{array}{l}\text { Gender and community dummy variables } \\
\text { only }\end{array}$ & 157.88 & -4.11 & 25.83 & 0.00 & 0.118 \\
\hline 4 & All community dummy variables & 161.58 & -2.31 & 27.64 & 0.00 & 0.097 \\
\hline 9 & $\begin{array}{l}\text { All community dummy variables, market } \\
\text { visitation }\end{array}$ & 160.39 & -1.41 & 28.53 & 0.00 & 0.104 \\
\hline
\end{tabular}

Only one model is within the best model subset denoted by delta $\mathrm{AIC}_{\mathrm{c}}$, and that model includes all predictor variables. The preferred model explains $30.8 \%$ of the variance in the data, and has an AIC weight which indicates that it is significantly more likely than the subsequent models to be correct for the data set. The complete multiple regression output is shown in Table 5.14 below:

Table 5.14: Multiple regression model for square root reef fish citation abundance under Model One

\begin{tabular}{|c|c|c|c|}
\hline & B & SE B & $\beta$ \\
\hline (Constant) & 2.285 & .387 & \\
\hline Age & -.011 & .005 & $-.186^{*}$ \\
\hline Gender $(0=$ Female, $1=$ Male $)$ & .322 & .142 & $.157^{*}$ \\
\hline $\begin{array}{l}\text { Birth locality ( } 0=\text { Local, } 1=\text { Non- } \\
\text { local) }\end{array}$ & -.222 & .202 & -.079 \\
\hline Educational attainment & .104 & .027 & $.316^{* *}$ \\
\hline $\begin{array}{l}\text { Linguistic preference }(0=\text { Bislama, } \\
1=\text { Vernacular })\end{array}$ & .037 & .217 & .012 \\
\hline Annual church attendance & .020 & .014 & .100 \\
\hline Annual visits to market & -.002 & .003 & -.047 \\
\hline $\begin{array}{l}\text { Commercial occupation }(0=\mathrm{No} \\
\text { commercial occupation, } 1=\text { Commercial } \\
\text { occupation) }\end{array}$ & .297 & .232 & .087 \\
\hline $\begin{array}{l}\text { Tisvel vs. Unmet ( } 0=\text { Unmet, Tisvel, } \\
\text { Dixon Reef, } 1=\text { Tisvel) }\end{array}$ & .854 & .205 & $.359 * *$ \\
\hline $\begin{array}{l}\text { Dixon Reef vs. Unmet ( } 0=\text { Unmet, } \\
\text { Tisvel, Lawa, } 1=\text { Dixon Reef) }\end{array}$ & .575 & .203 & $.229 *$ \\
\hline $\begin{array}{l}\text { Lawa vs. Unmet ( } 0=\text { Unmet, Dixon } \\
\text { Reef, Tisvel, } 1=\text { Lawa) }\end{array}$ & .560 & .191 & $.243^{*}$ \\
\hline
\end{tabular}

When converted from the square root values shown in the 'beta' column, I observe that an increase of five years in age is associated with citing 4.97 fewer folk species of reef fish; that males typically 
cite 6.8 more than females; and that, most notably, every five years spent in formal education tends to increase the abundance of reef fish citations by 7.87. Moreover, participants at Tisvel, Dixon Reef, and Lawa all created significantly more abundant freelists of reef fish than those at Unmet: by 9.85 , 8.18 , and 8.09 citations respectively.

\subsubsection{Ecological knowledge score}

Participants scored an average of $60.8 \%$ correct answers in the ecological knowledge test (mean $=$ $60.79, \mathrm{SD}=17.90$. Figure 5.7 outlines mean result for the four sections of the ecological knowledge quiz, and shows that in general mean scores were fairly evenly patterned. Overall, participants at Lawa scored higher than those at other communities, however differences between communities were not significant. Mean scores in the marine identification section of the quiz (including fish and shellfish identifications) were significantly higher at Lawa and Tisvel (for example, Lawa vs. Dixon Reef $\mathrm{t}=3.105, \mathrm{df}=81, \mathrm{p}<.05)$, and mean scores in the bird identification were significantly higher in Dixon Reef compared to Lawa $(\mathrm{t}=3.177, \mathrm{df}=81, \mathrm{p}=.002)$ and Tisvel $(\mathrm{t}=2.301, \mathrm{df}=75, \mathrm{p}<.05)$.

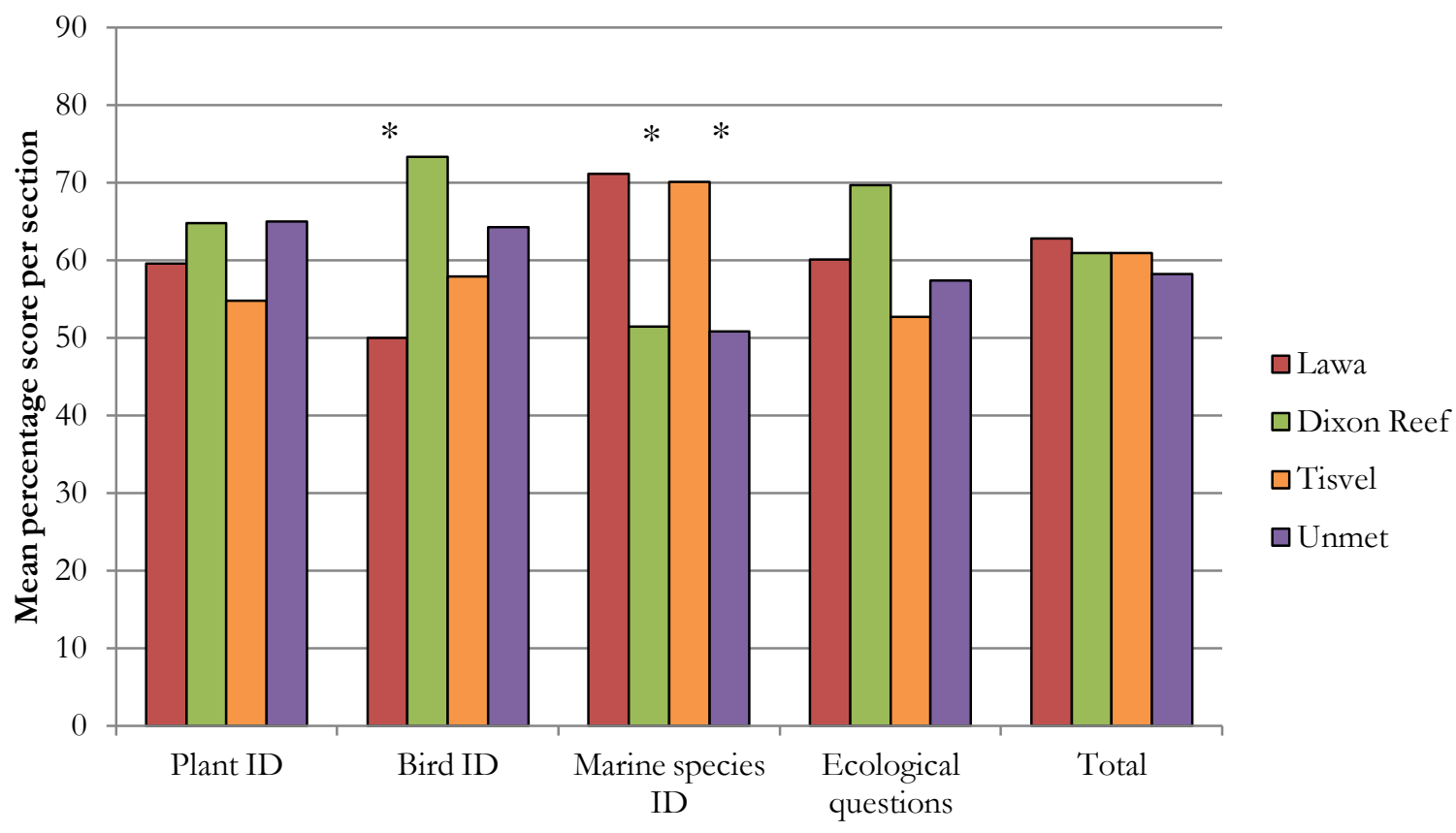

Section of ecological knowledge quiz

Figure 5.7: Mean percentage score per section of ecological knowledge quiz, per community. * = significant difference at $\mathrm{p}<.05$

Once again, individual scores displayed some of the patterns hypothesised in Table 5.1, and were significantly correlated with age $(r=.260, \mathrm{p}<.05)$, local birth $(\mathrm{r}=-.234, \mathrm{p}<.001 ; U=1171.5, \mathrm{p}$ $<.01)$, and preferential use of vernacular language $(\mathrm{r}=.365, \mathrm{p}<.001 ; U=764, \mathrm{p}<.001)$. Other patterns, however, differed from those hypothesised, and male gender displayed a significant association with percentage score in the ecological knowledge test $(\mathrm{r}=.369, \mathrm{p}<.001 ; U=1995, \mathrm{p}$ 
$<$.001). Gender difference varied between communities: at Lawa and Dixon Reef, males scored significantly more than females (respectively, $U=113.5, \mathrm{p}<.001 ; U=41.0, \mathrm{p}<.001$ ), however there were no significant differences at Tisvel and Unmet (Figure 5.8).

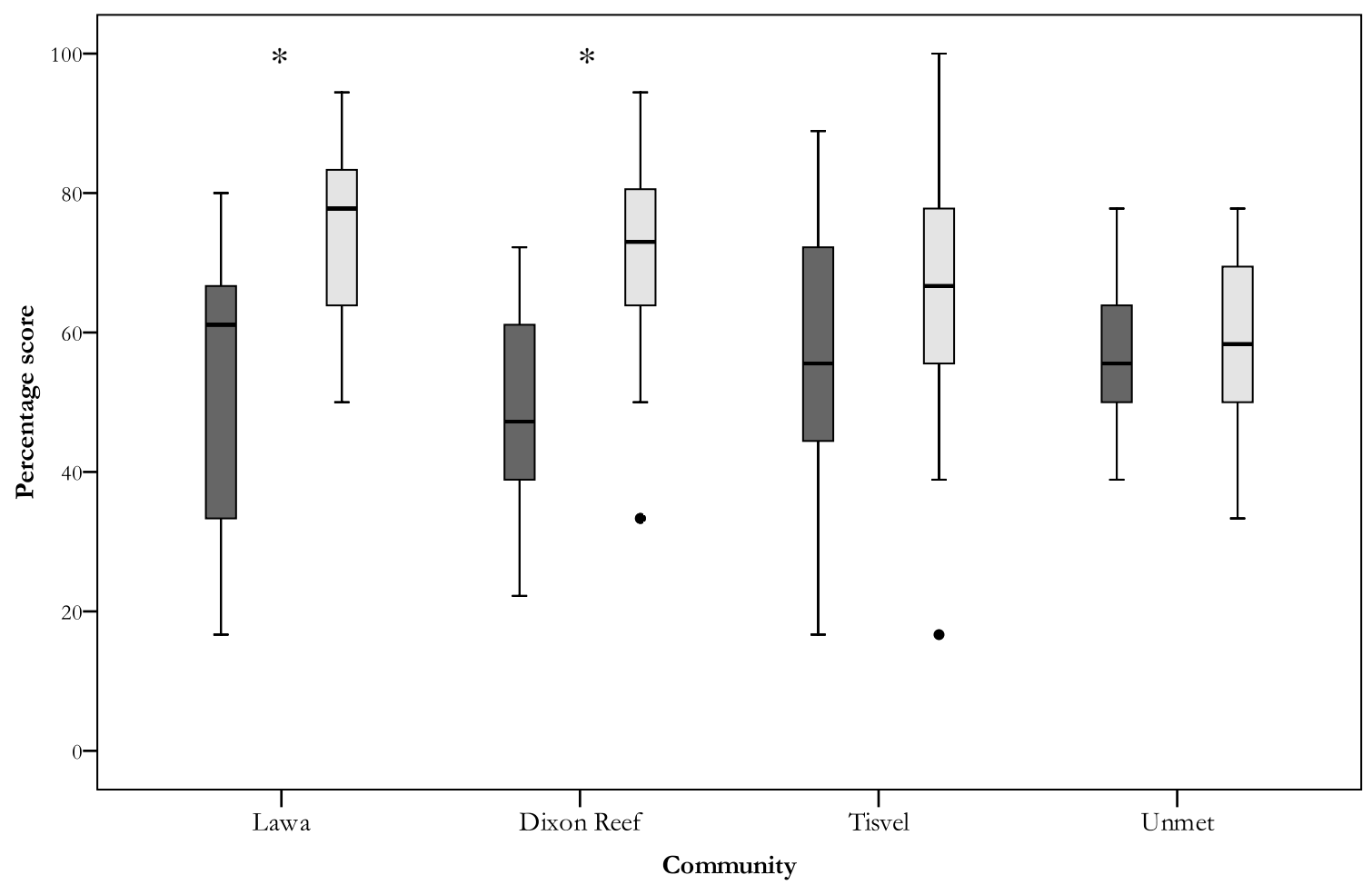

Figure 5.8: Scores in ecological knowledge quiz, per community and gender (light boxes indicate male participants, dark boxes indicate female participants; $*=$ differences between male and female significant at $\mathrm{p}<.001)$

\subsection{Model selection and multiple regression}

Scores in the ecological knowledge quiz were normally distributed and so were not transformed for multiple regression and model ranking procedures. Standard diagnostic plots did not reveal any issues, so I proceeded with AIC model-ranking procedure as described above (Table 5.15). 
Table 5.15: AIC model ranking for score in ecological knowledge test

\begin{tabular}{|c|c|c|c|c|c|c|}
\hline Hypothesis & Variables included & RSS & $\mathbf{A I C}_{\mathrm{c}}$ & $\Delta \mathrm{AIC}_{\mathrm{c}}$ & $\mathrm{AIC}_{\mathrm{c} \cdot \mathrm{W}}$ & $\mathbf{r}^{2}$ \\
\hline 1 & All variables & 33686.71 & 958.2712 & 0.00 & 0.54 & 0.366 \\
\hline 7 & $\begin{array}{l}\text { Age, gender, educational } \\
\text { attainment, linguistic } \\
\text { preference }\end{array}$ & 37224.50 & 960.20 & 1.93 & 0.21 & 0.300 \\
\hline 8 & $\begin{array}{l}\text { Age, gender, educational } \\
\text { attainment, linguistic } \\
\text { preference, market visitation }\end{array}$ & 36783.36 & 960.26 & 1.99 & 0.20 & 0.308 \\
\hline 2 & All demographic variables & 36019.40 & 963.21 & 4.94 & 0.05 & 0.322 \\
\hline 11 & $\begin{array}{l}\text { Gender and community } \\
\text { dummy variables only }\end{array}$ & 39939.66 & 970.51 & 12.24 & 0.00 & 0.249 \\
\hline 6 & Age, gender, education & 39242.77 & 973.91 & 15.64 & 0.00 & 0.262 \\
\hline 3 & Modernisation variables only & 42480.70 & 981.43 & 23.16 & 0.00 & 0.201 \\
\hline 5 & Age, gender & 45749.07 & 998.87 & 40.60 & 0.00 & 0.139 \\
\hline 10 & $\begin{array}{l}\text { All modernisation and } \\
\text { community dummy variables } \\
\text { only }\end{array}$ & 45545.27 & 1004.74 & 46.47 & 0.00 & 0.143 \\
\hline 4 & $\begin{array}{l}\text { Community dummy variables } \\
\text { only }\end{array}$ & 52696.94 & 1019.58 & 61.31 & 0.00 & 0.009 \\
\hline 9 & $\begin{array}{l}\text { Community dummy variables } \\
\text { and market visitation }\end{array}$ & 52468.21 & 1020.95 & 62.68 & 0.00 & 0.013 \\
\hline
\end{tabular}

Three models here fit within the 'best model' subset where $\Delta \mathrm{AIC}_{\mathrm{c}}<2$, and age, gender and educational attainment feature in each of them. However, given the disparity in AIC weights between the top ranked model and the next two, as well as the fact that both the second- and thirdranked models have $\triangle \mathrm{AIC}$ very close to 2 , I utilise hypothesis one as the preferred model. This model is able to explain $36.6 \%$ of the variance in the data, and is more than twice as likely as the next-ranked model to be correct for the data. The full regression model is displayed in Table 5.16. 
Table 5.16: Multiple regression model for percentage score in ecological knowledge quiz under Hypothesis One

\begin{tabular}{|c|c|c|c|}
\hline & $\mathbf{B}$ & SE B & $\beta$ \\
\hline (Constant) & 26.618 & 6.577 & \\
\hline Age & 0.263 & .083 & $.243^{*}$ \\
\hline Gender $(0=$ Female, $1=$ Male $)$ & 12.577 & 2.373 & $.352^{* *}$ \\
\hline Birth locality $(0=$ Local, $1=$ Non-local $)$ & -10.982 & 3.415 & $-.227^{*}$ \\
\hline Educational attainment & 1.086 & .464 & $.182^{*}$ \\
\hline $\begin{array}{l}\text { Linguistic preference }(0=\text { Bislama, } \\
1=\text { Vernacular })\end{array}$ & 15.286 & 3.591 & $.301 * *$ \\
\hline Annual church attendance & -.136 & .248 & -.037 \\
\hline $\begin{array}{l}\text { Commercial occupation }(0=N o \\
\text { commercial occupation, } 1=\text { commercial } \\
\text { occupation) }\end{array}$ & -.061 & .044 & -.097 \\
\hline Annual visits to market & -6.078 & 3.878 & -.103 \\
\hline $\begin{array}{l}\text { Tisvel vs. Unmet ( } 0=\text { Unmet, Tisvel, } \\
\text { Dixon Reef, } 1=\text { Tisvel) }\end{array}$ & 4.438 & 3.460 & .109 \\
\hline $\begin{array}{l}\text { Dixon Reef vs. Unmet }(0=\text { Unmet, } \\
\text { Tisvel, Lawa, } 1=\text { Dixon Reef) }\end{array}$ & -.290 & 3.560 & -.006 \\
\hline $\begin{array}{l}\text { Lawa vs. Unmet }(0=\text { Unmet, Dixon } \\
\text { Reef, Tisvel, } 1=\text { Lawa) }\end{array}$ & .486 & 3.248 & .012 \\
\hline
\end{tabular}

While all predictor variables were included in the model, only age, gender, birth locality, educational attainment, and preferential use of vernacular languages had a significant influence. Results indicate that a 20 year increase in age tends to increase ecological knowledge score by $5.3 \%$, and that men scored $12.6 \%$ more than women. Participants who were born locally scored nearly $11 \%$ more than those who had moved from elsewhere, and ecological knowledge also increases by over $1 \%$ for every year that the participant had been in formal education. Finally, participants who preferentially use vernacular languages attained scores of over 15\% higher than those who preferentially use Bislama.

\subsubsection{Skill proficiency}

Participants scored a mean of 9.13 out of a total of 18 possible, with a standard deviation of 2.73 .

Scores were patterned in a way which upheld some of the predictions of the hypotheses in Table 5.1, and were positively associated with age $(r=0.209, \mathrm{p}<.001)$, linguistic preference for vernacular languages $(\mathrm{r}=0.195, \mathrm{p}<.05 ; \mathrm{U}=1137.0, \mathrm{p}=.026)$, and residence at Dixon Reef $(\mathrm{r}=0.173, \mathrm{p}<.05$; $\mathrm{U}=1562.0, \mathrm{p}=.028)$. Again, males scored significantly higher than females $(\mathrm{r}=0.387, \mathrm{p}<.01 ; \mathrm{U}=$ 1831.0, $\mathrm{p}<.001)$. 


\subsection{Model selection and multiple regression}

Proficiency scores were normally distributed and so were not transformed for multiple regression and model ranking procedures. Standard diagnostic plots did not reveal any issues, so I proceeded with AIC model-ranking procedure (Table 5.17).

Table 5.17: AIC model ranking for proficiency scores

\begin{tabular}{|c|c|c|c|c|c|c|}
\hline Hypothesis & Variables included & RSS & $\mathrm{AIC}_{\mathrm{c}}$ & $\Delta \mathrm{AIC}_{\mathrm{c}}$ & $\mathrm{AIC}_{\mathrm{c} \cdot \mathrm{w}}$ & $\mathbf{r}^{2}$ \\
\hline 1 & All variables & 815.47 & 292.464 & 0.00 & 0.85 & 0.322 \\
\hline 7 & $\begin{array}{l}\text { Age, gender, education, linguistic } \\
\text { preference }\end{array}$ & 918.87 & 297.07 & 5.07 & 0.07 & 0.236 \\
\hline 8 & $\begin{array}{l}\text { Age, gender, education, linguistic } \\
\text { preference, market visitation }\end{array}$ & 908.22 & 297.25 & 5.25 & 0.06 & 0.245 \\
\hline 6 & Age, gender, education & 951.54 & 300.90 & 8.90 & 0.01 & 0.209 \\
\hline 5 & Age, gender & 968.73 & 301.84 & 9.84 & 0.01 & 0.195 \\
\hline 2 & All demographic variables & 968.73 & 303.96 & 11.96 & 0.00 & 0.195 \\
\hline 10 & $\begin{array}{l}\text { All modernisation \& community } \\
\text { dummy variables }\end{array}$ & 1041.77 & 327.40 & 35.40 & 0.00 & 0.134 \\
\hline 4 & All community dummy variables & 1113.95 & 327.84 & 35.84 & 0.00 & 0.074 \\
\hline 9 & $\begin{array}{l}\text { All community dummy variables, } \\
\text { market visitation }\end{array}$ & 1113.94 & 329.99 & 37.99 & 0.00 & 0.074 \\
\hline 3 & All modernisation variables & 1103.01 & 330.48 & 38.48 & 0.00 & 0.083 \\
\hline 11 & $\begin{array}{l}\text { Gender and community dummy } \\
\text { variables only }\end{array}$ & 1203.16 & 343.17 & 51.17 & 0.00 & 0.215 \\
\hline
\end{tabular}

Only one model fitted within the 'best model' subset, and includes all variables. This model is able to explain $32 \%$ of the variance in the data, and had an AIC weight that was much higher than the next best. The full multiple regression output is displayed in Table 5.18 . 
Table 5.18: Multiple regression for proficiency scores under Model One

\begin{tabular}{|c|c|c|c|}
\hline & B & SE B & Beta \\
\hline (Constant) & 3.801 & 1.055 & \\
\hline Age & .040 & .013 & $.245^{* *}$ \\
\hline Gender $(0=$ Female, $1=$ Male $)$ & 2.127 & .382 & $.390 * *$ \\
\hline Birth locality $(0=$ Local, $1=$ Non-local $)$ & .580 & .544 & .078 \\
\hline Educational attainment & .172 & .075 & $.186^{*}$ \\
\hline $\begin{array}{l}\text { Linguistic preference }(0=\text { Bislama, } \\
1=\text { Vernacular })\end{array}$ & 1.216 & .574 & $.156^{*}$ \\
\hline Annual church attendance & .046 & .039 & .083 \\
\hline Annual visits to market & -.004 & .007 & -.043 \\
\hline $\begin{array}{l}\text { Commercial occupation ( } 0=\text { No commercial } \\
\text { occupation, } 1=\text { Commercial occupation) }\end{array}$ & -1.252 & .656 & -.133 \\
\hline $\begin{array}{l}\text { Tisvel vs. Unmet ( } 0=\text { Unmet, Tisvel, Dixon } \\
\text { Reef, } 1=\text { Tisvel) }\end{array}$ & -.104 & .558 & -.017 \\
\hline $\begin{array}{l}\text { Dixon Reef vs. Unmet ( } 0=\text { Unmet, Tisvel, } \\
\text { Lawa, } 1=\text { Dixon Reef) }\end{array}$ & 1.305 & .579 & $.191 *$ \\
\hline $\begin{array}{l}\text { Lawa vs. Unmet }(0=\text { Unmet, Dixon Reef, } \\
\text { Lawa, } 1=\text { Lawa) }\end{array}$ & .972 & .525 & .162 \\
\hline
\end{tabular}

Most of the results from the multivariate regression here corroborate the initial correlations above. A year's increase in age was associated with a minor (0.04) increase in proficiency score; males typically scores 2.13 points higher than females; preferential speakers of vernacular scores 1.21 points higher than those who speak Bislama; and residence at Dixon Reef was associated with a score 1.31 points higher than at Unmet. One result adds to the correlational analysis however, with educational attainment associating significantly with proficiency score: each year of extra schooling is associated with a 0.17 increase in a participants overall score.

Please see Section 6.3.1 in the following chapter for further analysis of the proficiency data.

\subsubsection{Comparison of TEK measures}

Dependent variables were all correlated with each other, and in general those participants who score highly in one measure were likely to score highly in another (Table 5.19). The strongest correlations were between proficiency score and the medicinal plant measures; however, these were also strongly associated with background ecological knowledge. The weakest correlations were evident between reef fish citation abundance and the other dependent variables. 
Table 5.19: Bivariate Pearson's correlations between dependent variables

\begin{tabular}{lcccccc}
\hline & $\begin{array}{c}\text { Shannon- } \\
\text { Weiner }\left(\mathrm{H}^{\prime}\right)\end{array}$ & $\begin{array}{c}\text { Richness } \\
(\mathrm{Med})\end{array}$ & $\begin{array}{c}\text { Abundance } \\
(\mathrm{Med})\end{array}$ & $\begin{array}{c}\text { Abundance } \\
(\text { Fish })\end{array}$ & $\begin{array}{c}\text { Ecological } \\
\text { knowledge }\end{array}$ & $\begin{array}{c}\text { Proficiency } \\
\text { score }\end{array}$ \\
\hline Shannon-Weiner (H') & 1.000 & $.998^{* *}$ & $.980^{* *}$ & $.256^{* *}$ & $.435^{* *}$ & $.557^{* *}$ \\
Richness (Med) & & 1.000 & $.990^{* *}$ & $.252^{* *}$ & $.430^{* *}$ & $.550^{* *}$ \\
Abundance(Med) & & 1.000 & $.250^{* *}$ & $.445^{* *}$ & $.548^{* *}$ \\
\hline Abundance (Fish) & & & 1.000 & $.193^{*}$ & $.268^{* *}$ \\
Ecological knowledge & & & & 1.000 & $.372^{* *}$ \\
Proficiency score & & & & & 1.000 \\
\hline
\end{tabular}

$*=$ correlation significant at the 0.05 level; ${ }^{*}=$ correlation significant at the 0.01 level

NB: Dependent variables above the dashed line refer to medicinal plant knowledge

The different dependent variables displayed subtly different patterns in response to the predictor variables under multiple regression (Table 5.20). In the table below, 'NS' indicates that the interaction was not significant, and 'N/A' indicates the predictor variable was not included in the preferred model. ' + ' indicates a significant positive relationship (for categorical predictor variables, refer to legend for direction of relationship), and '-' reflects a significant negative relationship. 
Table 5.20: Summary of multiple regression results of best models for four dependent variables

\begin{tabular}{|c|c|c|c|c|c|c|c|c|c|c|c|}
\hline & Age & Gender & $\begin{array}{l}\text { Birth } \\
\text { locality }\end{array}$ & $\begin{array}{c}\text { Educational } \\
\text { attainment }\end{array}$ & $\begin{array}{l}\text { Church } \\
\text { attendance }\end{array}$ & $\begin{array}{l}\text { Linguistic } \\
\text { preference }\end{array}$ & $\begin{array}{l}\text { Market } \\
\text { visits }\end{array}$ & $\begin{array}{l}\text { Commercial } \\
\text { occupation }\end{array}$ & $\begin{array}{l}\text { Unmet vs. } \\
\text { Tisvel }\end{array}$ & $\begin{array}{l}\text { Unmet vs. } \\
\text { Dixon Reef }\end{array}$ & $\begin{array}{l}\text { Unmet vs } \\
\text { Lawa }\end{array}$ \\
\hline Richness (Med) & NS & + & $\mathrm{N} / \mathrm{A}$ & NS & $\mathrm{N} / \mathrm{A}$ & + & - & $\mathrm{N} / \mathrm{A}$ & $\mathrm{N} / \mathrm{A}$ & $\mathrm{N} / \mathrm{A}$ & $\mathrm{N} / \mathrm{A}$ \\
\hline $\begin{array}{l}\text { Shannon-Weiner } \\
\left(\mathrm{H}^{\prime}\right)\end{array}$ & NS & + & $\mathrm{N} / \mathrm{A}$ & NS & $\mathrm{N} / \mathrm{A}$ & + & $\mathrm{N} / \mathrm{A}$ & $\mathrm{N} / \mathrm{A}$ & $\mathrm{N} / \mathrm{A}$ & $\mathrm{N} / \mathrm{A}$ & $\mathrm{N} / \mathrm{A}$ \\
\hline Abundance (Fish) & - & + & NS & + & NS & NS & NS & NS & + & + & + \\
\hline $\begin{array}{l}\text { Ecological } \\
\text { knowledge }\end{array}$ & + & + & + & + & NS & + & NS & NS & NS & NS & NS \\
\hline Proficiency score & + & + & NS & + & NS & + & NS & NS & NS & + & NS \\
\hline
\end{tabular}

NB: Categorical variables coded as above: for Gender, positive sign indicates correlation with male gender; for Birth Location, positive sign indicates local birth; for Linguistic Preference, positive sign indicates preference

for vernacular; for Commercial Occupation, negative sign indicates no commercial occupation; for Community dummy variables, sign refers to Lawa, Dixon Reef, or Tisvel against Unmet 


\subsection{Discussion}

These results highlight the diversity and depth of ethnobiological knowledge still present in the four focus communities. In terms of medicinal plants, the 237 species identified by participants account for nearly 20\% of the c. 1200 plants in Vanuatu (Cabalion et al. (1991), cited in Bourdy and Walter (1992)), and places these communities near the top of the table compiled by Begossi (2002), which compares richness of medicinal plants in tropical areas. This is impressive given that freelisting was only undertaken across ten illness domains, and especially so given the Jack1 richness estimator was some way from reaching asymptote (Figure 5.5). This indicates that the sample of ten illness domains was incomplete, and that were I to keep sampling in additional illness domains, I would be likely to continue uncovering new species for some time.

The richness of medicinal plant usage from these four communities is not surprising, given importance of ethnomedical knowledge for healing within the focus communities. One factor likely to influence the persistence of ethnomedical knowledge is the difficulty of access to comprehensive western medical care for three of the four communities, which makes the retention of traditional medicinal knowledge and practice a necessity. Moreover, participants commonly noted that western medical care, whilst largely effective, was unable to treat a large range of illnesses that were perceived to have a traditional aetiology (such as the snek spirit illness in Table 5.3), which could only be identified by local doctors and healed using traditional methods. As such, these patterns corroborate qualitative evidence from informants that herbal and traditional plant knowledge retains an important and necessary role in everyday life. Moreover, this study may indicate support for the findings of others that the assumption of a dualism between western medical care and traditional medicine is not always correct, and rather that the two systems are often complimentary (Furusawa 2006; McGrath 1999; Straume 2009).

\subsubsection{Drivers of variability in TEK on Malekula}

I find that TEK is variably patterned within the focus communities according to the key predictor variables. In this section I discuss the influence of each of the predictor variables in turn.

\subsubsection{Gender}

One of the more consistent results was that men score more highly in all four predictor variables than women. This runs counter to my hypotheses, which predicted that there would be no strong gender difference. For medicinal plant knowledge it is especially surprising, as piloting and post-facto interviews with expert informants (both men and women) noted that both genders were typically equally responsible for healing the ten focus illnesses within the community. Some studies in other 
areas of the globe have noted that women cite more medicinal plants than men, as they are often responsible for the bulk of household and everyday healing (Begossi et al. 2002; Caniago and Siebert 1998; Voeks and Leony 2004).

However, these findings agree with previous studies from South America, the Pacific, and Africa , all of which have found that men list more plants than women (Case et al. 2005; Hanazaki et al. 2000; Kristensen and Balslev 2003; Stagegaard et al. 2002). This result could be interpreted in a number of ways. It may be the case that it reflects societal norms within the focus communities, which may favour the role of men and give them a greater role in healing and the transmission of medicinal knowledge. Gender relations are an ongoing site of dialogue in Vanuatu; however some actors (including the national council of chiefs or Malvatumauri) have adopted a position that identifies the 'kastom' role of women to be one of 'quiet subservience' (Regenvanu 2005: see Section 4.4). This position that has been debated by a number of prominent authors (see, for example, Bolton 2003; Molisa 1980; Regenvanu 2005); however, were it to be true in the focus communities it may result in women having lower stocks of medicinal plant knowledge. Alternatively, it may indicate that women's plant knowledge is eroding more rapidly than men's, although I can deduce no significant evidence for this from this research.

Interestingly, differences between men and women were consistently stronger at Lawa and Dixon Reef than at Tisvel and Unmet. I speculate further on community-level drivers of variation in medicinal plant knowledge below, however this disparity is especially striking given we might expect that the recent geographic shift from the interior to the coast has differentially impacted on male knowledge. Research has consistently suggested that men tend to be more familiar with the ethnobotany of old-growth forested habitats, and especially arboreal species, whereas women tend to be more informed about disturbance species associated with homegardens, swiddens, and other products of human habitat change (Caniago and Siebert 1998; Coe and Anderson 1996; Voeks 2007). We might expect that the most profound differences in terms of plant assemblages between the interior and the coast is likely to be in the former, rather than the latter, habitat, as communities would be able to shift with essential herbs and plants from home gardens. As such, the geographic shift to the coast may have resulted in men needing to re-learn a large proportion of their ethnomedical knowledge. In Lawa and Dixon Reef, which have been longer-established at the coast, the male body of knowledge has had longer to become acquainted with the coastal habitat; this trend may have resulted in the observed variation in TEK between men and women.

It is also possible that this result is an artefact of sampling procedure, on two fronts. Firstly, in terms of medicinal plants, it is possible that the ten illness domains measured may not be domains in which women are especially familiar. In some of the only ethnomedical research to have been undertaken in 
Vanuatu, Bourdy and Walter (1992) found that women had extensive herbal medicinal knowledge for all stages of the reproductive cycle, and note that this domain was one that was typically attended to only by women. In not surveying this, and other similar domains, I may have inaccurately measured the scope of women's knowledge on Malekula. Secondly, it is possible that the entire interview process was biased towards men. I found, as have other similar studies (e.g. Case et al. 2005), that in interview piloting women were often uncomfortable and would attempt to answer as briefly as possible to speed along the interview process (perhaps reflective of the power differential discussed in Section 3.2. Although I attempted to ameliorate this issue by employing trained local female research assistants to interview all female participants, interviewees would have known the provenance of the interview (i.e. me), and as such may have remained uncomfortable. Moreover, men may have been more inclined than women to try and impress the interviewer by naming more species (Case et al. 2005), thus inflating the apparent gender bias.

\subsubsection{Age}

For medicinal plants, age did not significantly associate with individual-level richness or diversity of medicinal plants cited, nor was it a significant factor in either of the multiple regression models. As such, there was no support for my initial hypotheses at this stage, which stated that older participants would have a greater body of knowledge than younger. However, when group-level diversity indices were calculated and age was split into three age cohorts, significant differences were evident between the oldest (aged 61+) and youngest (aged between 18 and 30) cohorts. This indicates that the older generation cite plants that are richer in terms of species number, and with a higher evenness (relative abundance) than the younger; and as such, that the younger age cohort are drawing on a body of knowledge that is more homogenous than that of the older. This is corroborated by Figure 5.3, which indicates that the oldest age cohort cite a significantly lower proportion of cultivated plants (or, a higher proportion of wild plants) than younger cohorts. Similar findings have been recorded by Ross (2002b), who found greater agreement in the knowledge of younger people amongst the Lacandon Maya; and by Zent and Zent (2004), who noted that homogenisation of ethnobiological knowledge was occurring apace in nucleated communities.

As noted in Section 2.4.1, some studies have cautioned against utilising associations between age and ethnobotanical knowledge to infer diachronic loss of TEK from synchronic data (Godoy et al. 2009; Voeks and Leony 2004). Whilst this method may accurately describe the distribution of knowledge amongst a population, it may also provide a misleading impression of knowledge erosion, as it can be confounded by normal age accumulation curves (Godoy et al. 2009). Moreover, estimating loss of knowledge through associating level of TEK with age is difficult, as changes in knowledge between cohorts might simply reflect changes in other variables (such as in abundance of wildlife, formal 
schooling, and opportunities for migration) that are difficult to account for. Ideally, studies of this type would utilise longitudinal datasets, and interestingly the two studies to do this both recorded surprising persistence of ethnobotanical knowledge (van Etten 2006; Zarger and Stepp 2004).

However, given that most adults can be expected to have acquired the bulk of their theoretical medicinal plant knowledge by their late teens (Godoy et al. 2009), the significant differences in the type of plants being cited between age cohorts is an important result. It indicates that the geographic and cultural shifts of the past 50 years have resulted in a reduction in the richness of plant knowledge, possibly facilitated by geographic shifts from the bush to the coast. In other words, there is likely to be a process of TEK homogenisation occurring within the youngest age cohort, which is reason for concern given the importance of TEK on Malekula. There are also significant methodological implications, which I discuss further below.

In this light, the finding that citation abundance reef fish displays a moderately strong and highly significant negative association with age is interesting, as it indicates that knowledge of reef environment is increasing amongst the younger generation. This result highlights the importance of historical and geographic factors in the variation of TEK. Each of the focus communities arose from the founding of mission stations between 1950 and 1970 (Section 4.7 for more information), during which time community members moved to the coast from traditional lands in the interior of the island. Therefore, there may not have been stocks of 'traditional' knowledge of reef fish within these communities. For the younger generation, aided by increased mobility and connectedness, and growing up in a coastal environment, inshore marine resources have a greater relevance than they do to the older generations. Therefore, they have a greater incentive and ability to learn this knowledge. If theoretical knowledge of plants remains largely constant after age 20 (Godoy et al. 2009), and one might expect the same to be true for folk knowledge of reef fish, stressing the importance of the local environment in learning ethnobiological knowledge at a young age.

This result is an important reminder of the dynamism of TEK, and concurs with research by Guest (2002) who found that under certain conditions ecological knowledge may increase. The author used these findings to question the validity of terms such as 'traditional' when discussing the ecological knowledge of local people. As noted by Godoy et al (2009): "If ethnobiological knowledge is dynamic, then one must be open to the possibility that it may increase, and not that it inevitably wanes" (p 64).

Methodological constraints may affect the reliability of this finding. Primarily, focussing on abundance of citations and not identifying reef fish using scientific nomenclature potentially confounds the data by making it impossible to distinguish between different levels of classification. Whilst many Bislama reef fish names are polytypic, and refer to families of reef fish (for example, 
'bluefish' or 'whitefish'), others (such as 'long mouth', or Hyporbamphus affinus) are unitypic and refer to particular species. This issue may be inflated by reporting bias - young men may have a greater propensity to list many species in order to impress the interviewer (Case et al. 2005), and so might have intentionally listed several levels of classification for one species of fish. However, I argue that the likely effects from this are small, and that given that citation abundance a legitimate output of freelist interviewing (cf. Quinlan 2005), that this result still represents an interesting trend. Clearly, however, there is scope for further detailed research.

It is also interesting that participant scores in the ecological knowledge test positively associate with age across all sections of the test. Most notably, this association was evident in a significant (yet weak) correlation between age and scores in the marine section of the ID quiz (shellfish and reef fish; $r=$ $0.266, \mathrm{p}<.01)$. Clearly, then, older participants are able to recognise marine species well even if they are not able to name them in a freelist. Given that the analysis above considers scores to be correct only if identifications were in vernacular language, it is possible that this result simply reflects the elder generation's greater linguistic proficiency. However, if the multiple regression analysis is re-run using a 'coarse' filter (where identifications were marked correct if given in Bislama), the model loses some predictive power $\left(\mathrm{r}^{2}=0.335, \mathrm{p}<.001\right)$ however the key explanatory variables remain significant and have comparable effect sizes. Therefore I can say with some confidence that older people are more proficient at the species identification than the younger generation. I note, however, that the freelist measures a much broader range of knowledge than the limited scope of the ecological knowledge quiz.

It is also worth noting that ID tests based around photographs are likely to be biased against older participants, as they are commonly less used to printed materials, may have poor eyesight, or may be used to identifying plants by smell or feel (Case et al. 2005; Godoy et al. 1998). It was evident during data collection that for some older participants the ID section of the quiz was difficult, and I can therefore infer that the effect size is under-reported in this particular case.

\subsubsection{Educational attainment}

Contrary to predictions, educational attainment was positively associated with all alternative measures of TEK, though it displayed no significant association with individual-level richness and diversity of medicinal plant citations. This is surprising: as noted, the formal school system has been identified as a key driver of TEK erosion in Vanuatu (Regenvanu 2005), and as such I expected that a negative relationship would be evident. Similar findings have been reported in Reyes-García et al. (2005) in the Bolivian Amazon, who suggest that increased time spent in school allows the chance for increased interaction with other pupils and thus facilitates the sharing of knowledge. This seems a 
plausible scenario in the focus communities as well, particularly with regards to knowledge of folk species of reef fish. Pupils who attend secondary school typically move away from their communities and live in large dormitories (McCarter, unpublished field notes). This allows the interaction of children from different communities, including those from small island communities which are likely to have a far richer knowledge of the marine environment.

Moreover, once again, the test format may also favour participants who are used to a school environment, in particular the completion of written tests. As such, this result may not reflect actual knowledge, but rather proficiency at the sample method. This is corroborated by the significant categorical differences in the diversity of cited medicinal plants by those who had more than six years of formal education against those who had less, indicating that although richness and abundance of citations are not significantly different, more highly educated people as a group cite a lower diversity of species. This is not altogether unexpected, given the amount of time that high school students spend away from their home villages, and is in line with my hypotheses.

\subsubsection{Linguistic preference}

Linguistic preference was a consistently important variable in my analyses, and participants who preferentially use vernacular language, as opposed to Bislama, cited a higher richness and diversity of plants (both individually and as a group), as well as scoring more highly in the ecological knowledge quiz. The latter result may seem self explanatory, as answers were marked 'correct' only if given in the vernacular language. When tested using the coarse filter (thus accepting all correct answers in Bislama or vernacular) there remains an evident association between linguistic preference and broader ecological knowledge $(r=0.25, \mathrm{p}<.001)$.

These results represent an important finding, as linguistic loss may be most salient threat to traditional knowledge and practice, both in Vanuatu (Vari-Bogiri 2005) and in the global context (Harmon 2002; Nettle and Romaine 2000). In Mexico, Benz et al. (2000) found that empirical knowledge of plant use is more diverse and evenly shared amongst the vernacular-speaking Huastec people than between mestizo and Spanish-speaking populations in the same area. Because vernacular languages on Malekula are locally adapted and evolved, they encapsulate a detailed local knowledge of the environment (including names of medicinal plants), and learning vernacular language is likely to be intrinsically linked to becoming literate in the local flora and fauna. Bislama, on the other hand, lacks the in-depth vocabulary with which to name, discuss, and conceptualise the natural world (Crowley 2003; MacClancy 2002).

These findings corroborate the scoping study presented in Section 4.9, which found that linguistic loss is perceived by a range of community members to be a key driver of TEK erosion. In the local 
context, linguistic change is being driven by two key factors: exogamous marriage between two people of differing linguistic groups, which results in Bislama being spoken in the home environment; and a lack of support for vernacular languages within the school system. Similar factors are evident across Vanuatu, and some 25 of the nation's 106 languages are extinct or moribund (Lynch and Crowley 2001). These factors have resulted both in the spread of Bislama as the preferential means of communication, and the spread of certain vernacular languages (such as Novol at over EastCentral Malekula) at the expense of other languages. The status of vernacular language education in Vanuatu is the site of an ongoing debate (cf. Crowley 2000; Shipman 2008; Siegal 1996; Stahl 2004), and the fact that these results empirically link linguistic preference and level of TEK should give added impetus to calls for its support by the formal school system.

\subsubsection{Market integration}

The two indicators of market integration, annual visits to market and commercial occupation, were notable for their overall lack of significant impact on levels of TEK. For commercial occupation, this may be explained fairly easily - as noted, economic opportunities are scarce and very few Malekulans devote themselves entirely to any one occupation. Therefore, they probably do not need to specialise to the degree that would impact on their contact with the environment and/or level of TEK. Annual visits to market also did not prove to bear significant influence on overall ecological knowledge or abundance of citations of reef fish.

Interestingly, however, annual visits to market did negatively associate with richness and diversity of medicinal ethnobotanical knowledge, a result that was supported at a categorical level by the use of diversity indices. Moreover, because the communities were situated along a gradient of ease of access to market, the significant differences in medicinal species diversity between Lawa/Dixon Reef and Tisvel/Unmet further corroborates the hypothesis that ease of access to market has a negative influence on the ethnomedical knowledge of participants. Whilst the expansion of the market economy has been linked to the erosion of ethnobotanical knowledge, other research has noted complex patterns whereby integration into markets impacts on TEK only within certain, non-useful, domains (Benz et al. 2000; Godoy et al. 1998; Reyes-García et al. 2005; Reyes-García et al. 2007b; Ross 2002a). In terms of ethnomedical knowledge, the most pertinent factor here is likely to be the medical facilities that are available at Lakatoro/Norsup. Therefore, the observed patterns may be due to increased access to, and use of, western biomedical facilities. Research in other areas has found that the direct competition of western biomedicine has resulted in lower rates of intergenerational transmission of ethnomedical knowledge, or increased rarity or disappearance of specialist healers within the community (Begossi et al. 2002; Case et al. 2005; Lozada et al. 2006; Zent and Maffi 2009). 
Further research which specifically investigates usage rates of hospital and clinical facilities against ethnomedical knowledge would be needed to clarify this relationship.

\subsubsection{Community differences}

Finally, I noted patterning of TEK across different communities. For individual level analysis, Unmet scored significantly lower than the other communities in terms of citation abundance of reef fish. Unmet is the most recently established community, and before 1972 inhabitants were situated in the north-western interior plateau at Amokh. As such, this finding seems to be a plausible result of the recent geographic shift, as I would not expect participants who had spent their formative years in the forest environment to have a deep knowledge of the coast. Indeed, I find that the negative relationship between age and reef fish citation abundance is significantly stronger at Unmet than at other communities $\left(\mathrm{r}_{\mathrm{s}}=-.630, \mathrm{p}<.001\right)$, indicating perhaps that the rate of learning about the marine environment is most rapid at Unmet. Clearly, cultural norms and values (not just proximity to the resource) are important, and throughout the interview process at Unmet participants emphasised that they were not 'man solvota',22 and had no culture (and, as such, no interest in learning about) of the ocean. However, given that most learning of theoretical knowledge occurs in the early-mid teens, I might expect there to be a rapid knowledge accumulation curve after relocation, and might expect that were this survey to be repeated in 20 years the accumulation curve would have reached asymptote.

Individual-level richness and diversity of medicinal plant citations did not differ significantly between communities, however community-level diversity and expected citation richness at Unmet and Tisvel was significantly lower than at Dixon Reef. As noted, this may be a function of ease of access to market or hospital resources. It is also likely that the geographic shift described in the previous paragraph has played an important role in reducing community-level diversity and richness of medicinal ethnobotanical knowledge, as plant species assemblages on the coast are significantly different from those in the interior (Rosillo-Cale and Woods 2003). The community at Dixon Reef, having been located on the coast for much longer than at Unmet, may have simply had longer to learn the flora of the new environment. Moreover, bodies of medicinal plant knowledge may adapt more slowly that knowledge of reef fish, and so may take longer to adapt to the new geographic environment. Elsewhere in the world, ethnomedical knowledge is commonly transmitted through vertical (parent to child) means (e.g. Lozada et al. 2006), whereas we might hypothesise that knowledge of reef fish - being a recent addition to the corpus of traditional knowledge - will be spread horizontally (or within a peer group), and will thus evolve more quickly (Hewlett and CavalliSforza 1986). Chapter Six considers the cultural transmission of TEK in more detail.

\footnotetext{
${ }^{22}$ Literally, people of the sea
} 


\subsubsection{Comparison and methodological issues}

There were several conflicting trends across the six dependent variables, the most obvious of which being the positive association age with abundance of reef fish citations against the higher diversity of medicinal plants cited by older age cohorts, and the higher scores in the ecological knowledge quiz by older participants. These data highlight the complexity of inferring changes in ethnobiological knowledge from synchronic survey results, and the importance of choosing an appropriate proxy to measure TEK. Most studies aiming to measure TEK use ethnobotanical knowledge, in some form, as a proxy for wider knowledge (Reyes-García et al. 2007a). These findings indicate that different trends can be evident between different domains (i.e. plants and fish) and dimensions (i.e. theoretical and practical) of knowledge, despite identical interview techniques and samples. Therefore my findings add weight to recommendations from a recent review (Reyes-García et al. 2007a) that research into TEK be broadened to other ecological domains, as different domains of knowledge do not necessarily display similar patterns.

The relatively strong correlations between the different proxies of TEK (Table 5.19) do indicate that the different knowledge domains are linked. That is, while the different TEK proxies might react different to some of the variables associated with modernisation, they tend to display similar patterns overall. This is not entirely surprising: as well as reflecting the pervasive influence of explanatory variables such as gender, we might also expect that individuals who are proficient at one survey methodology might be proficient at another. Most interesting, however, are the relatively strong correlations between proficiency score and the other measures of TEK. Although the proficiency score was created using a somewhat blunt methodology (Section 5.2.4.3), it gives a basic measure of practical skills and thus reflects a different dimension of knowledge to the other dependent variables, which only measure theoretical knowledge. Despite the fact that practical skills stem from theoretical knowledge (cf. Godoy et al. 2009), the link between these dimensions has not been shown consistently in the literature: for example, work in Patagonia has found no evidence that theoretical and practical ability are linked (Ladio and Lozada 2004). Follow-up research is needed to confirm this finding; however this result represents an important contribution to the ethnobiological literature.

The finding that older age cohorts cite a higher diversity of plants than younger groups highlights the importance of thoroughly analysing quantitative information. In this research, the use of diversity indices was critical to forming my conclusions. If I, as in previous studies (e.g. Quinlan and Quinlan 2007), had only focussed number of medicinal citations, I would have missed a key trend in the data - namely, that older people appear to hold a richer and more diverse body of knowledge than younger. Moreover, I note that it is important to check for the linearity of trends in the data. Multiple 
regression techniques assume linearity, and so the use of age cohorts (c.f. Voeks and Leony 2004) to identify non-linear trends allowed us to recognise thresholds that existed between groups.

It is also important to note the limited predictive power of the models generated through the AIC procedure, in particular multiple regressions for medicinal plant knowledge that left around $82 \%$ of the variance in the data unexplained. These results are consistent with other studies of human resource use, and highlight the difficultly in predicting individual-level variation in knowledge using only a limited set of predictor variables (e.g. Gavin and Anderson 2007; Godoy et al. 1998). Moreover, there may be a number of confounding variables (such as those associated with personal attributes such as resourcefulness, personal interest, exactness, and attention to detail) which are difficult to quantify and measure, and may impact on the strength of the model.

Several methodological issues (including the potential for gender and age biases) have already been noted above. The freelisting method has two further important constraints, as discussed in Quinlan (2005) and Quinlan and Quinlan (2007). The first is that people may know more species than they list: as noted, some medicinal plant knowledge is secret on Malekula, and some participants noted they held back answers on the basis that the information was culturally or commercially sensitive. Second, although freelisting taps active knowledge, it is possible that informants were citing medicines and plants for which they did not have the associated practical knowledge to prepare, and without a secondary practical test this effect is impossible to detect. These issues should not, however, impact too greatly on the richness and abundance measures used to test hypotheses in this chapter.

\subsection{Conclusions}

This chapter synthesised key methods from the ethnobiological literature to gain an understanding of the factors driving variation of TEK on Malekula Island. In particular, I focussed on elucidating the socio-demographic predictors of knowledge of medicinal plant species and folk species of reef fish; examined non-linear trends between categorical variables, using diversity indices; and triangulated results using an ethnobiological quiz across four domains of knowledge and a broad assessment of skill proficiency. These data indicate that TEK on Malekula is patterned by demographic variables, mainly gender; that indicators of modernisation, such as annual visits to market and linguistic preference, evidently have some influence on individual accumulation of TEK; and that drivers of variation in TEK appear to influence different domains of TEK is different ways.

These results may have important policy implications for those interested in the maintenance and revitalisation of TEK in Vanuatu. First, linguistic preference appears to be a key variable in 
patterning TEK on Malekula. Despite the small sample size for the Bislama-speaking group, these data are robust and should give added weight to calls for vernacular language to be supported by the school curriculum. Given the sheer diversity of Vanuatu, such a move would be unlikely to involve tailored solutions for every language group; and as such, could focus on utilising resource men and women within the community for the teaching of vernacular language (this may have other important educational benefits too, some of which are discussed in Chapter Seven). Because of the intrinsic links between vernacular language and TEK this would automatically confer the benefit of increasing the transmission of TEK. This result also highlights the value of the ongoing work of the VCC and their efforts to facilitate the inclusion of TEK and vernacular language into the science curriculum (Baeraleo 2010).

Second, evidence have shown that that the geographic shifts of the past 50 years may have resulted in the elder age cohort having unique knowledge of the interior bush environment that may not be being transmitted to the younger. I emphasise here that many interviewees in the younger age cohorts demonstrated detailed ethnomedical knowledge; however that this body of knowledge is more evenly shared and less species-rich that that of the elder age cohorts. Efforts at TEK revitalisation might therefore profit from focussing on detailing, discussing, and transmitting the ethnobotanical knowledge of the interior environment through the use of bush camps and field trips, and ensuring that idiosyncratic and diverse knowledge bases are not lost for posterity.

Third, these data remind us of the dynamic nature of culture and TEK. Geographic and cultural shifts may result in some domains of knowledge decreasing, whilst others increase. As such, these findings add weight to recent calls in the ethnobiological literature for an increased breadth of investigation that account for multiple domains and dimensions of TEK (cf. Reyes-García et al. 2007a). If, as some authors recommend (e.g. Zent and Zent 2004), biocultural conservation programs are to be based around detailed local assessments of TEK vitality, these assessments must be broad enough in their scope to allow for differential rates of change between domains. In a culturally diverse area such as Melanesia, the requisite detail of such projects may render them impractical, and new approaches may be needed.

Finally, these findings remind us of the extraordinary vibrancy and depth of TEK on Malekula and the need for an active interest in its maintenance and revitalisation. Detailed medicinal plant and ecological knowledge such as these play an important role in maintaining adaptive capacity and socioecological resilience amongst human populations (Berkes and Folke 1998). In particular, stores of medicinal plant knowledge represent an important source of self-sufficiency and relative independence from market centres such as Lakatoro/Norsup. Given that pressures toward cultural homogenisation and increased environmental risk are likely to increase in coming years, an ongoing 
interest in the area from academics and policy makers is critical to the continued viability of traditional knowledge systems. Moreover, it is important that research programs have an applied component which seeks to actively facilitate the maintenance of TEK. Community resource books produced as part of this research program (Appendix 11.13) may offer one example of a means to do this - however there needs to be an increased research focus on TEK conservation to ascertain the effectiveness of the various proposed methodologies. I address some of these issues in Chapters Seven and Eight. 


\section{The cultural}

\section{transmission of TEK on}

\section{Malekula}

\subsection{Introduction}

The literature and evidence presented in the Introduction (Section 2.4) and Chapter Five have contributed to our understanding of the ways in which TEK is patterned within and between communities. Typically, this work has examined variation in TEK by surveying TEK holders and analysing the distribution of knowledge according to demographic and social variables (e.g. Benz et al. 2000; Quinlan and Quinlan 2007; Reyes-García et al. 2005). These analyses have allowed scholars to infer broader patterns of cultural change, erosion, or continuity, and comment on how modernisation and globalisation may be causing the observed distributions of TEK.

However, such analyses are able to say very little about how TEK holders within these research programs came to acquire their knowledge (Reyes-García et al. 2009). This is a critical issue because TEK, as with all facets of culture, is perpetuated and sustained by processes of cultural transmission (Ohmagari and Berkes 1997). If we do not understand the ways in which actors in a social network acquire and disburse information, we are unable to comment on the mechanisms that influence patterns of variation (Ross 2002a; Zarger and Stepp 2004). Importantly, we are also unable to comment on the processes that may be driving change or erosion in TEK (Cristancho and Vining 2009). Therefore, to understand how TEK as a lived body of knowledge exists within its source culture, there is a need to understand how it is transmitted within communities.

Studies of cultural transmission will also be critical for applied programmes seeking the maintenance and revitalisation of TEK. A key task of these programs is the re-opening of traditional lines of transmission, commonly between elder and younger generations (Florey 2009). However, within any body of cultural knowledge there is likely to be significant variation in terms of how TEK is 
transmitted (Zent and Maffi 2009). For example, there are likely to be different strategies for transmission of different domains of knowledge, or there may be generational differences in how actors within a social network acquire knowledge (Case et al. 2005; Ross 2002b). Therefore, to maximise the effectiveness of future programmes for TEK maintenance, they should be founded on a thorough understanding of suitable modes of cultural transmission within the focus community (Bates 2009).

This chapter addresses the cultural transmission of TEK on Malekula, with the aim of contributing to theoretical knowledge of the cultural transmission of TEK and how it might be changing in smallscale rural societies. I use a mixed-methods approach to outline the cultural transmission pathway of 18 TEK skills and discuss perceptions of intergenerational changes in the transmission of TEK on Malekula. In the remainder of this section, I introduce the theoretical framework of cultural transmission and recent work within ethnobiology, before proceeding to outline my approach to this issue.

\subsubsection{Cultural transmission: theoretical background}

Cultural transmission refers to the "...process of social reproduction in which the culture's technological knowledge, behaviour patterns, cosmological beliefs, etc., are communicated and acquired.” (Hewlett and Cavalli-Sforza 1986: p 922). The cultural transmission of knowledge and skills is embedded within wider processes of socialisation and acculturation. Although a complete review of the ways in which culture structures human development is outside the scope of this chapter, I note here that processes of cultural transmission and acculturation have been a critical facet of anthropological work for several decades (Atran and Sperber 1991; Boyd and Richerson 1985; Cavalli-Sforza and Feldman 1981; Lave and Wenger 1991; Vygotsky 1978).

Authors have hypothesised that the cultural transmission of knowledge and skills can occur via at least three pathways (Table 6.1). These are from parent to child (vertical transmission); between members of the same generation (horizontal transmission); and from non-parental individual members of the parental generation to members of the filial generation (oblique transmission) (Cavalli-Sforza et al. 1982; Reyes-García et al. 2009). Oblique transmission itself can take two forms: when one person transmits information to many people of a younger generation (one to many), or when a novice acquires skills from older adults other than ones parents (many to one) (Cavalli-Sforza and Feldman 1981). The three pathways are not mutually exclusive, and will vary across domains of knowledge according to demographic and other characteristics (Cavalli-Sforza et al. 1982). Moreover, the transmission pathway can vary across an individual's lifetime. For example, although parents can play a critical role in knowledge transmission at an early age, other sources of information (such as an 
individual's peer group) play a more prominent role at later stages (Aunger 2000; Hewlett and Cavalli-Sforza 1986).

The pathway through which knowledge is transmitted affects the evolutionary characteristics of that body of knowledge (Reyes-García et al. 2009). Vertical transmission tends to be highly conservative, and to assist and maintain variation within social groups. Vertical transmission is usually associated with slower rates of cultural change and evolution, compared with horizontal and oblique modes, because models of strict vertical transmission are less susceptible to influence from outside sources (Hewlett and Cavalli-Sforza 1986). Therefore, innovation is unlikely if no other modes of transmission are present within the population (Lozada et al. 2006). In contrast, horizontal transmission is associated with the rapid spread of innovations and an easy uptake of new technologies. Horizontal and oblique transmissions involving many transmitters to one receiver tend to generate the highest uniformity within the social group (Hewlett and Cavalli-Sforza 1986; Reyes-

García et al. 2009).

Table 6.1: A model of cultural transmission (after Hewlett and Cavalli-Sforza 1986)

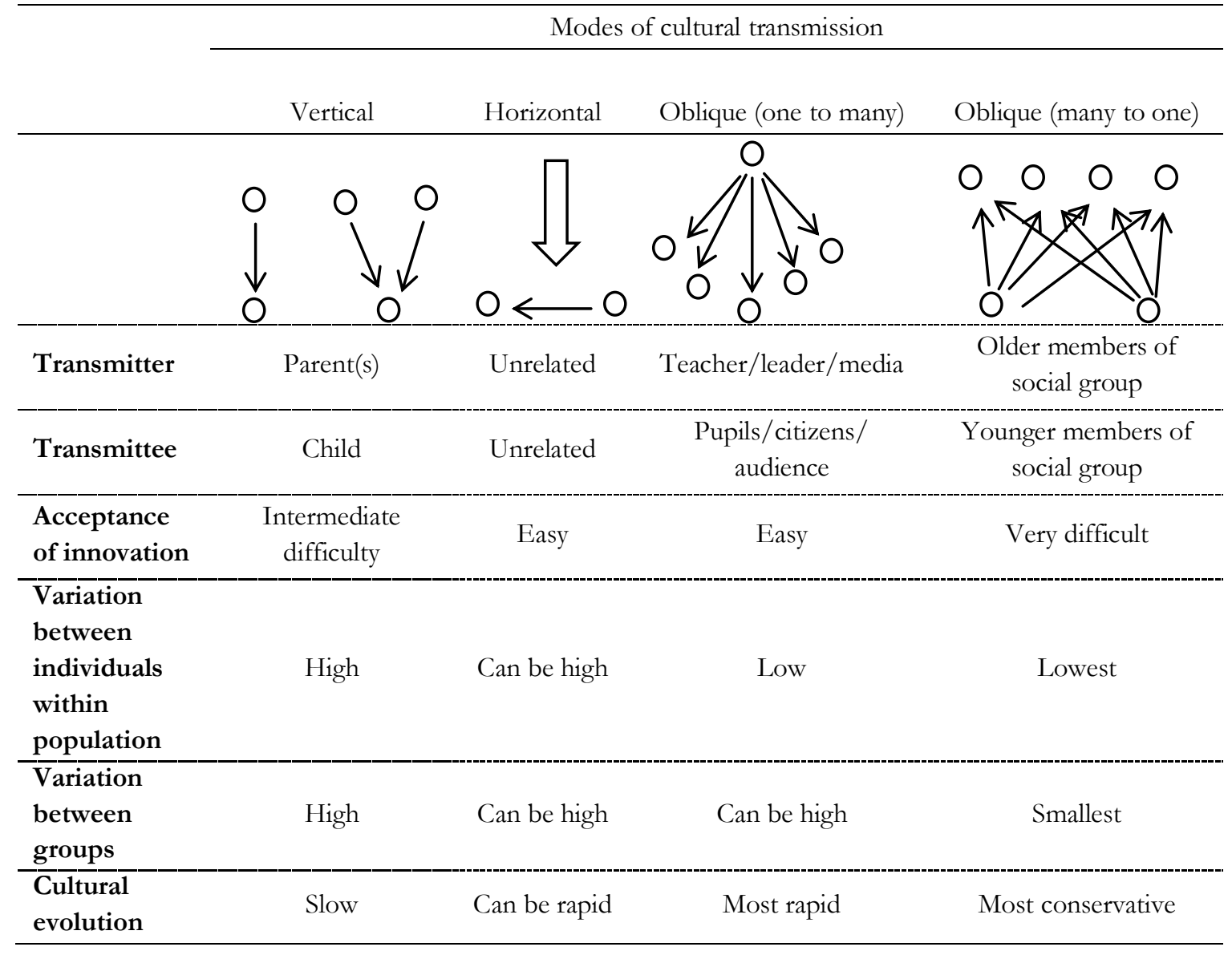

Broadly speaking, cultural transmission and human development are adapted to both ecological and cultural contexts, which Super and Harkness (1986) propose combine to form a metaphorical space 
they call the 'developmental niche' (DN). The DN includes the physical and social settings the child is exposed to, customary child-rearing strategies, and the folk theories regarding how children can thrive. I return to the DN in Section 6.2.3.3. The cultural transmission strategies employed by human societies can change over time, because in different ecological and social conditions different socialisation patterns are necessary to prepare children for adulthood (Greenfield and Lave 1982; Greenfield et al. 2000). Consequently, dominant modes of cultural transmission can shift, resulting in profound differences in the nature of knowledge within social networks (Vygotsky 1978). For example, Greenfield et al. (2000) document diachronic shifts in weaving style within a Mayan community in response to large-scale social and environmental change. They suggest that “...each ecology emphasises a different set of skills, different developmental pathways, and different processes of socialisation or informal education" ( $p 353)$.

\subsubsection{Cultural transmission of TEK}

Although studies specifically focussing on the cultural transmission of TEK are lacking, a small body of literature has been published utilising two broad approaches. Several authors (such as Ohmagari and Berkes (1997)) have used quantitative methods to survey a range of skills and assess their transmission pathways. Others (such as Lancy (1996)) have taken an ethnographic approach that is deeply grounded within focus communities. These two methodological approaches have emphasised differing aspects of TEK transmission (Zent and Maffi 2009).

Quantitative research indicates that vertical transmission of TEK is often dominant (Casagrande 2002; Eyssartier et al. 2008; Hewlett and Cavalli-Sforza 1986; Lozada et al. 2006; Ohmagari and Berkes 1997), sometimes overwhelmingly so. For example, Hewlett and Cavalli-Sforza (1986), in work amongst the Aka pygmies in Central Africa noted that vertical transmission was dominant for $80 \%$ of the practical skills they surveyed. In particular, several authors stress the role of the mother in passing on medicinal and linguistic ability (Cruz García 2006; Garro 1986; Lozada et al. 2006). Vertical transmission is particularly pronounced at an early age, in part because of parent-child proximity and attachment (Hewlett et al. 2002). Importantly, Aunger (2000) argues that the role of vertical transmission may be overstated in some studies because research participants may be biased in the recall or social acknowledgement of transmission events (normative response bias).

Quantitative studies also indicate that horizontal and oblique transmission pathways can play a key role in transmission of some TEK domains and at certain life stages. Horizontal transmission has been noted in cases where technologies have been recently introduced into communities, or in nonstable and new environments. For example, Hewlett and Cavalli-Sforza (1986) found that horizontal transmission was dominant for crossbow construction, a technique that had been recently introduced 
to the area. Similarly, Casagrande (2002) noted that Tzeltal Mayan men shared a significant amount of ethnomedical knowledge with peers in the lowland farms where they worked as seasonal labourers. In other situations, oblique transmission is important, in particular in domains where specialised knowledge is concentrated with experts and trained resource people (Ohmagari and Berkes 1997; Reyes-García et al. 2009). This is often the for ethnomedical knowledge: for example, Lozada et al. (2006) note that the influence of local experts in medicinal knowledge transmission in Patagonia, but observed that the importance of such actors has declined in recent years. Similarly, in a unique study amongst the 'Tsimane' (Brazil) Reyes-García et al (2009) found the strongest concordance in ethnobotanical knowledge and skills between the filial generation and the older age cohort (rather than the parental age cohort), indicating that transmission of this domain is mostly oblique.

Qualitative work, on the other hand, emphasised the specific relationships that regulate the flow of ethnoecological information, and has situated these relationships within the broader process of acculturation and socialisation (Zent and Maffi 2009). Several previous studies stressed the role of play and peer-learning (horizontal transmission) in the development of skills and knowledge, noting that it often occurs in informal settings without strict instruction from any one individual (Lancy 1996; Zarger 2002). Children and peers may take increased responsibility for the instruction of younger friends and siblings from an early age, until such time as teaching responsibility shifts to authority figures (Maynard 2002; Maynard 2004). In other situations, horizontal transmission can become more dominant with age; as the social context of actors changes from family to peer groups, so does the source of learning (Aunger 2000; Rogoff 1981). Skills are reinforced through repeated practice, rather than being simply acquired through one-time observations (Ruddle and Chesterfield 1977).

In addition to vertical, horizontal, and oblique pathways, TEK acquisition can be experiential in nature. This has been stressed by research indicating that TEK acquisition is based on sustained contact with nature and with the land (Atran and Sperber 1991; Bates 2009; Nabhan 1998). In this sense, knowledge is 'learned' from the land rather than actively 'taught' by other actors (Bates 2009). This knowledge can be acquired through everyday activities such as play and through tasks such as the gathering of wild food resources (Setalaphruk and Price 2007; Zarger and Stepp 2004); or through experience built up over long engagement with the environment, such as hunting (Bates 2009). The timing of this learning process of critical importance, because the acquisition of TEK knowledge and skills usually begins at an early age: children have been noted to attain adult competency at ethnomedical plant identification between ages 12 and 14 (Hunn 2002; Stross 1973; Zarger 2002; Zarger and Stepp 2004). This is different for different dimensions of TEK: whilst theoretical knowledge has been shown to peak by the late teens (Godoy et al. 2009), learning of complex skills such as hunting can take longer (Hewlett and Cavalli-Sforza 1986). 
Although the nature of the transmission of TEK is a critical facet of the health of TEK systems, there have been few empirical ethnobiological studies to assess how systems of TEK transmission change in response to social and environmental changes (Zarger and Stepp 2004). This is an important gap because aspects of modernisation, such as the introduction of widespread formal schooling, can introduce different and competing modes of knowledge transmission (Bates 2009; Ohmagari and Berkes 1997). New modes of knowledge transmission may have a variety of impacts, such as introducing new forms of knowledge; changing dominant channels of TEK transmission; or by changing the age at which skills are acquired (Ohmagari and Berkes 1997). These changes impact on the characteristics of this body of knowledge. For example, formal educational models may remove children from the setting for TEK transmission at an early age, there is a risk that this may result in 'acquisition deprivation' (Zent and Zent 2004). This is important, as the experiences of childhood may greatly impact on adult cognition and expertise with the biophysical world. As such, a lack of environmental experience may lead to less environmental knowledge and a lower inclination toward pro-environmental behaviour (Chipenuik 1995; Nabhan 1998; Zarger 2002).

In summary, two key issues emerge from this review of the literature. The first is general uncertainty around how TEK is transmitted within rural communities, primarily stemming from a lack of targeted research attention. Indeed, as noted by Zarger and Stepp (2004): “... we have more information on what adults know than how they came to know it" (p 414). Like studies of TEK variation reviewed in Chapter Five, the studies that exist have rarely been situated in Oceania: indeed, there are no quantitative studies of cultural transmission based in the region. The second is a lack of understanding of how these transmission systems might change in response to the indicators of modernisation analysed in the previous chapter (Section 5.1.1). If we are to understand the process of knowledge erosion or homogenisation, then we must understand how systems of cultural transmission change. Changes in these systems influence the size and direction of shifts in broader culture, and ultimately result in the erosion or persistence of TEK.

In this chapter I add to the literature on both issues by employing a mixed methods approach in four communities on Malekula Island, Vanuatu. The specific objectives are to:

1. Examine the cultural transmission (the transmission pathway, the age of transmission, and the place of transmission) of TEK skills on Malekula

2. Investigate changes in the cultural transmission of TEK skills on Malekula 


\subsection{Methods}

\subsubsection{Setting}

Research for this chapter was based in the same four focus communities as in Chapter Five: Unmet, Tisvel, Dixon Reef, and Lawa. Please see Section 4.8 for more information

\subsubsection{Mixed methods procedure and ethics}

The two objectives outlined above necessitate a mixed methods approach, because quantitative and qualitative methodologies have inherent strengths and weaknesses (Bernard 1998). Quantitative approaches, such as structured interviews, are more effective at gaining a sample size for systematic comparison, but are limited in their ability to infer diachronic change and gain nuanced information (Creswell and Plano Clark 2007). Qualitative approaches, such as semi-structured interviews allow depth of analysis and are useful for gauging perceptions of change, but are more difficult to attain large sample sizes for objective comparison (Bernard 1998). Moreover, quantitative and qualitative approaches may yield different data on cultural transmission, as noted above (Zent and Maffi 2009). Therefore, I used an exploratory mixed methods research procedure, and collected data using both structured and semi-structured interviews.

Data were collected sequentially in two phases (Figure 6.1). First, I conducted detailed semistructured interviews with 18 expert informants in four focus communities. Next, I used a structured interview to examine three aspects of the transmission of the 18 key skills amongst 165 lay participants in four focus communities.

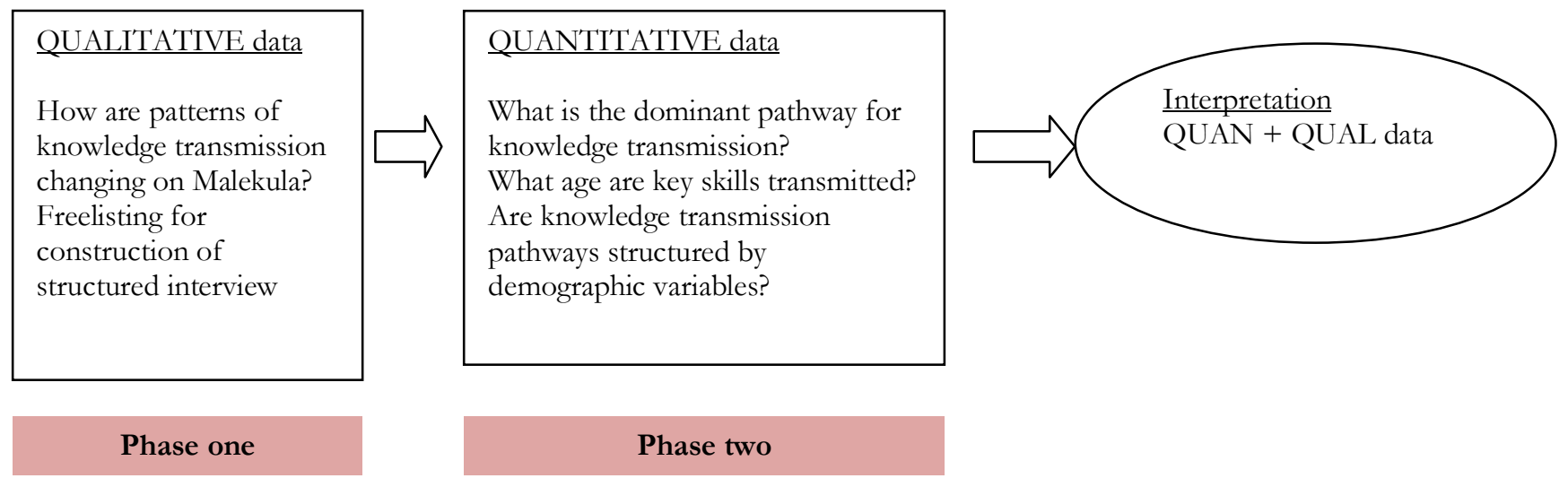

Figure 6.1: Schematic diagram of data collection methods and integration 
In the results section of this chapter I first present quantitative data from the structured interview, before contextualising these using the outcomes of the semi-structured interviews with TEK experts. I accord equal weight to both streams of data, as they are addressing different and distinct research questions.

For both sections of field research, I adhered to the code of ethics described in Section 3.3.2. All participants were adult, fully informed and offered their verbal consent to the interview process. All interview data remain confidential, and due to ongoing intellectual property concerns, binomial scientific names of medicinal or sacred plants will not be listed in this or any other publication.

\subsubsection{Phase one: Semi-structured interviews}

\subsubsection{Participants}

Phase one consisted of detailed semi-structured interviews with 18 expert informants. Participant selection was purposive and non-random. I began interviews with a gatekeeper informant in each community who was also a VCC fieldworker (Section 0), and asked the fieldworker to recommend community members who were expert in ethnobiological skills and knowledge. I recognise that this may bias results as it is reliant on the social networks of the gatekeeper informant, and so may restrict access to only a particular sector of the community (Bernard 1998). However, it is a useful means of gaining contacts in a relatively short timeframe, and was the only practicable method for sample selection.

Four of the interviewees were VCC fieldworkers; seven were chiefs; four were schoolteachers; and others held positions within the church or within community bodies. 14 of the interviewees here were male, and four were female. This gender imbalance was a result of the bias associated with snowball sampling, as well as with a common reluctance from some expert women to participate in the research. Such experiences have been recorded elsewhere in Melanesia (c.f. Case et al. 2005), and for the structured interview I attempted to ameliorate the issue by working with trained local female research assistants. Participant ages ranged between 29 and 69, with a median age of 50. Nine of the interviewees were from Dixon Reef, with three from each of the other focus communities. Interviews were conducted in Bislama, lasted between 30 and 90 minutes, and were translated only for reproduction in this chapter.

\subsubsection{Data collection}

The interviews had two aims: a) to gain a qualitative understanding of the nature and changes of ethnobiological knowledge transmission in the focus communities; and b) to create a structured 
interview for use in the wider community. Towards the first aim, I interviewed the expert informants during fieldwork between 2009 and 2010. Questions were loosely based on an interview schedule (Appendix 11.7), however the format allowed the flexibility to follow leads and interesting stories where the occasion arose. Key questions involved the nature of knowledge transmission pre-contact, including primary transmitters of knowledge, location, and technique; current modes of knowledge transmission; and the drivers of change in systems of knowledge transmission.

Toward the second aim, I asked interviewees to freelist ethnobiological skills that they considered will be important in their community in the future. Separate freelists were conducted within five use domains. The use domains were derived during workshops during 2008, where 36 participants from Malekula were asked to produce freelists of forest use categories. ${ }^{23}$ These categories were then sorted using pile sorts and hierarchical clustering (with the aid of Anthropac (Borgatti 1996)) to derive final use categories: construction, food, medicine, carving (e.g. slit drums, handicrafts, hunting implements) and weaving (e.g. mats, fans, rope). This method of deriving use categories was preferred over preinterview designation of use categories as it is based on in situ perceptions of forest use, and as such aims to reduce researcher bias (Gavin and Anderson 2007).

Skills were checked for saliency in each location through cross-checking with gatekeeper informants (VCC fieldworkers) that they were currently, or previously, practiced in that community. All skills that met this criterion were included in the interview for phase two (Table 6.3). I stress that these skills do not represent an exhaustive list of important TEK skills within the focus communities, and are intended as a representative assortment to provide an indication of the process of knowledge transmission on Malekula. Moreover, most of the skills themselves represent several individual skills: for example, herbal medicine is here treated as one skill, when in reality the body of ethnomedical plant knowledge on Malekula is substantial (see Chapter Five); as has weaving fans, mats, and baskets, each of which are woven in a range of designs representing different levels of difficulty.

\subsubsection{Data analysis}

The analysis for the first section above focussed on discussing the transmission of TEK knowledge and skills on Malekula, and paid particular attention to identifying nodes of intergenerational change in TEK transmission systems. I coded interview data based on suggestions from Miles and Huberman (1994) and Bernard (1998), in three distinct phases.

\footnotetext{
23 These workshops were a collaboration between the VCC and Victoria University and formed a parallel research program during 2008. They sought to document the variation of TEK from around Malekula, but are not substantively connected with this research
} 
First, I conducted a thorough scan of the 18 interview transcriptions, and annotated each with initial coding schema and suggestions. These were then compiled to derive an outline coding schema.

Second, I identified the most salient themes from the schema by sorting the initial themes into categories and subcategories. Themes were then compared with the Developmental Niche (DN) framework, which was proposed by Super and Harkness (1986; 2002) and utilised to study intergenerational changes in TEK transmission by Cristancho and Vining (2009). The DN is a psychological model which proposes that cultural transmission has environmental, socio-cultural, and individual aspects. As such it provides a useful superstructure on which to categorise factors influencing intergenerational changes in TEK transmission (Cristancho and Vining 2009). I fitted my key themes to the framework and noted the level of support for each from the 18 transcripts.

Third, I re-scanned the transcriptions and identified frequently occurring sentiments and unusual or dissenting views. The latter responses were tagged and noted for consideration. Results from the analyses are presented in two ways: first, in a summary table (Table 6.8), and through the use of exemplar quotations to support major themes.

\subsubsection{Phase two: Structured interviews}

\subsubsection{Participants}

I conducted structured interviews as part of the same structured interview as that used for Chapter Five (Appendix 11.9). The sample size for this section (165 participants) was slightly less than that for the previous chapter because on 12 occasions time restrictions precluded this section of the interview (sample details in Table 6.2). Participant selection was as described in Section 5.2.2.

Table 6.2: Participants for structured interview on TEK transmission

\begin{tabular}{lcccccc}
\hline & \multicolumn{3}{c}{ Age cohort } & \multicolumn{2}{c}{ Gender } \\
& & $18-30$ & $31-60$ & $60+$ & Male \% & Female \% \\
\hline Total & 165 & 59 & 85 & 21 & 49.4 & 50.6 \\
Lawa & 47 & 10 & 26 & 11 & 46.8 & 53.2 \\
Dixon Reef & 35 & 17 & 14 & 4 & 58.8 & 41.2 \\
Tisvel & 42 & 18 & 21 & 3 & 50 & 50 \\
Unmet & 41 & 14 & 24 & 3 & 43.9 & 56.1 \\
\hline
\end{tabular}




\subsubsection{Data collection}

The structured interview was undertaken as part of a broader survey (see Chapter Two). I first derived a subset of 18 key TEK skills using the procedure described in section 6.2.3.2 above. These skills are displayed in Table 6.3.

Table 6.3: 18 key skills included in structured interview

\begin{tabular}{|c|c|c|c|}
\hline Domain/skill & Short description & Commonly used species & Gender \\
\hline \multicolumn{4}{|l|}{ Construction } \\
\hline Pinning thatch & $\begin{array}{l}\text { Removing central mid-vein from leaves of } \\
\text { natangora palm; folding over spar of bamboo; } \\
\text { pinning with a short needle of bamboo. }\end{array}$ & Metroxylon warbugbii, Bambusia spp. & Mixed \\
\hline $\begin{array}{l}\text { Cutting posts for } \\
\text { house using an } \\
\text { axe }\end{array}$ & $\begin{array}{l}\text { Selection of appropriate timber, cutting post for } \\
\text { house using an axe (i.e. in opposition to the } \\
\text { 'modern way' of using a chainsaw) }\end{array}$ & $\begin{array}{l}\text { Various: including Macaranga } \\
\text { dioica, Intsia bijuga, Kleinhovia } \\
\text { hospita, Cordia alliodora }\end{array}$ & Mixed \\
\hline $\begin{array}{l}\text { Tying thatch } \\
\text { with vines }\end{array}$ & $\begin{array}{l}\text { Tying down panels of thatch using appropriate } \\
\text { vines from the bush (i.e. not using nails or rope) }\end{array}$ & $\begin{array}{l}\text { Various, including Entada } \\
\text { phaseoloides }\end{array}$ & Mixed \\
\hline $\begin{array}{l}\text { Weaving walls of } \\
\text { bamboo }\end{array}$ & $\begin{array}{l}\text { Splitting bamboo tubes of appropriate size; } \\
\text { weaving into walls of varying designs }\end{array}$ & Bambusia spp. & Mixed \\
\hline \multicolumn{4}{|l|}{ Food } \\
\hline Laplap wota & $\begin{array}{l}\text { A traditional form of laplap (pudding) made } \\
\text { without cooking in slow-flowing water }\end{array}$ & $\begin{array}{l}\text { Taro (Colocasia sp.), Yam (Dioscorea } \\
\text { sp.) }\end{array}$ & Female \\
\hline $\begin{array}{l}\text { Catching } \\
\text { freshwater } \\
\text { crayfish by hand }\end{array}$ & $\begin{array}{l}\text { Catching freshwater crayfish without the aid of } \\
\text { mask, snorkel, or wire (commonly used to spear) }\end{array}$ & $\mathrm{N} / \mathrm{a}$ & Mixed \\
\hline Yam medicine & $\begin{array}{l}\text { Use of traditional medicine to ensure good yam } \\
\text { growth }\end{array}$ & Various, confidential & Male \\
\hline Fish poison & $\begin{array}{l}\text { Using the fruit of common coastal trees to make } \\
\text { a poison which kills reef fish }\end{array}$ & Barringtonia racemosa & Mixed \\
\hline \multicolumn{4}{|l|}{ Medicine } \\
\hline Herbal medicine & A large variety of ethnomedical usages of plants & Various, confidential & Mixed \\
\hline \multicolumn{4}{|l|}{ Carving } \\
\hline Carving tamtam & Slit drums for ceremony and communication & $\begin{array}{l}\text { Intsia bijuga, Pterocarpus indicus, } \\
\text { Artocarpus altilus }\end{array}$ & Male \\
\hline Carving nalnal & Clubs used for ceremony & Various, including Intsia bijuga & Male \\
\hline $\begin{array}{l}\text { Cutting bow and } \\
\text { arrow }\end{array}$ & $\begin{array}{l}\text { Construction of bow and arrow, commonly used } \\
\text { for play and hunting }\end{array}$ & $\begin{array}{l}\text { Various, including Ficus sp., } \\
\text { Rhizophora stylosa }\end{array}$ & Male \\
\hline Carving canoe & Construction of canoe and outrigger & $\begin{array}{l}\text { Various, including Gyrocarpus } \\
\text { americanus, Intsia bijuga }\end{array}$ & Male \\
\hline \multicolumn{4}{|l|}{ Weaving } \\
\hline $\begin{array}{l}\text { Making a grass } \\
\text { skirt }\end{array}$ & Weaving of a grass skirt for use in ceremony & Various, including Pandanus sp. & Female \\
\hline Weaving mat & Weaving of large mats & Pandanus sp. & Female \\
\hline Weaving basket & Weaving of baskets, in a variety of designs & Pandanus sp. & Female \\
\hline Weaving fan & Weaving of fans, in a variety of designs & Pandanus sp., Cocos nuciflora & Female \\
\hline Weaving rope & $\begin{array}{l}\text { Using coconut fibre to weave rope, commonly } \\
\text { used on canoes }\end{array}$ & Cocos nuciflora & Mixed \\
\hline
\end{tabular}


The far right column of Table 6.3 indicates the gender of each skill. Ni-Vanuatu society is to some extent structured by gender, and men and women have defined roles (Bolton 2003; Curtis 2002). When cross-checking for skill saliency as described above I also asked the gatekeeper informants if the skills had a gendered component. In the list above, eight skills were classed as 'mixed', meaning that they were commonly conducted by both men and women; and five skills were conducted by only men and only women. 'Male' skills were typically defined as those within the use domain of carving, as well as some secret information associated with promoting yam growth; whereas 'female' skills were typically those involving the use domains of weaving and food preparation.

To obtain data I adapted the methods of Hewlett and Cavalli-Sforza (1986) and Ohmagari and Berkes (1997). For each skill, I asked participants if they had ever been taught the skill (i.e.

experienced at least one transmission event); and if so, who was the major teacher, at what age did it occur, and if there was a particular place for teaching that skill.

\subsubsection{Data analysis}

I analysed the quantitative data in two stages. First, I looked at transmission of the 18 key skills used in the structured interview, and compared with the individual-level proficiency analysed in the previous chapter (Section 5.3.2.3). I also analysed whether transmission pathway correlated with overall proficiency or other measures of TEK, in order to comment on the role that specific transmission pathways might play in structuring individual levels of TEK Second, I analysed the transmission pathway for each skill (who is the primary teacher, at what age, and where), and looked for variation according to basic demographic data (gender, age, and community). Categorical comparisons were conducted using student's t-tests, Wilcoxon Signed Rank tests and Freidman's non-parametric ANOVA depending on data distribution (normality). All data were analysed using SPSS 17.0 for Windows (SPSS Inc. 2008).

\subsubsection{Methodological limitations}

Three major methodological limitations constrain this study. First, the quantitative data here are reliant on self-reports (for example, when participant's believed that they had first been shown the skill), and are, thus, contingent on the memory of the interviewees. Especially in the case of the oldest interviewees (many of whom do not know their exact age), this is likely to be an unreliable metric (Case et al. 2005). Second, the nature of the learning process is likely to be more complex than can be accounted for by this method. Because I asked about active transmission events for each skill, I may not have accounted for experiential learning of the type which, in reality, may be the means through which most learning is achieved (Hunn 2002; Lancy 1996; Zarger 2002). Moreover, because each skill is, in effect, a complex of theoretical knowledge and practice, the various pieces may have 
been acquired earlier, and not recalled as such by participants. Finally, some transmission methods (such as vertical transmission) may be more memorable than other means, meaning that they will be over-represented in the sample transmission pathway. These constraints impinge on the resolution with which I am able to discuss cultural transmission. The chosen methods are, however, sufficient to shed some light on cultural transmission on Malekula and extend the current state of knowledge.

\subsection{Results}

Data are presented in two sections. First, I address objective one of this paper by presenting quantitative data on the cultural transmission of TEK in the focus communities. Second, I address objective two by presenting results of semi-structured interviews into changes in cultural transmission on Malekula.

\subsubsection{Cultural transmission in the focus communities (structured interviews)}

I outline the quantitative data according to three key aspects of cultural transmission: the transmission pathway (i.e. vertical, horizontal, oblique, and other forms of transmission), the age of first skill transmission, and the place where this skill transmission was reported to have taken place.

\subsubsection{Skill transmission and proficiency}

The 164 participants in the survey recorded 1,390 transmission events across the 18 key skills, an average of just over 77 per skill. All skills were recognised by all participants, except for the making of 'laplap wota', which was not understood by four male participants at Unmet. For gender specific skills (as specified in Table 6.3) percentages are calculated from the eligible gender only (male $\mathrm{n}=81$, female $n=83$ ). Occasionally, members of the opposite gender reported transmission of a gendered skill (for example, young men noting that they knew how to weave a mat). These responses were not common however $(\mathrm{n}=17$ or $1.2 \%$; over half of which $(\mathrm{n}=9)$ were females noting they could construct bow and arrows).

On average, participants reported transmission events at 8.5 of the 18 skills. Transmission events were unevenly distributed across the 18 skills (Figure 6.2), with a high percentage of participants reporting transmission of some skills (such as pinning thatch for houses $(92.7 \%)$ and weaving mats (97.6\%) and a very low percentage reporting transmission of others (such as making laplap wota $(21.7 \%)$ and carving tamtam (30.5\%)). The colours of each bar indicate the use domain of the skill. In particular, skills with primarily ceremonial importance (such as carving tamtam or nalnal) or those associated with pre-contact norms or spirituality (such as yam medicine and laplap wota) have a 
significantly lower percentage of participants reporting transmission events than the overall mean (combined, $\mathrm{t}=3.225, \mathrm{p}=.005)$.

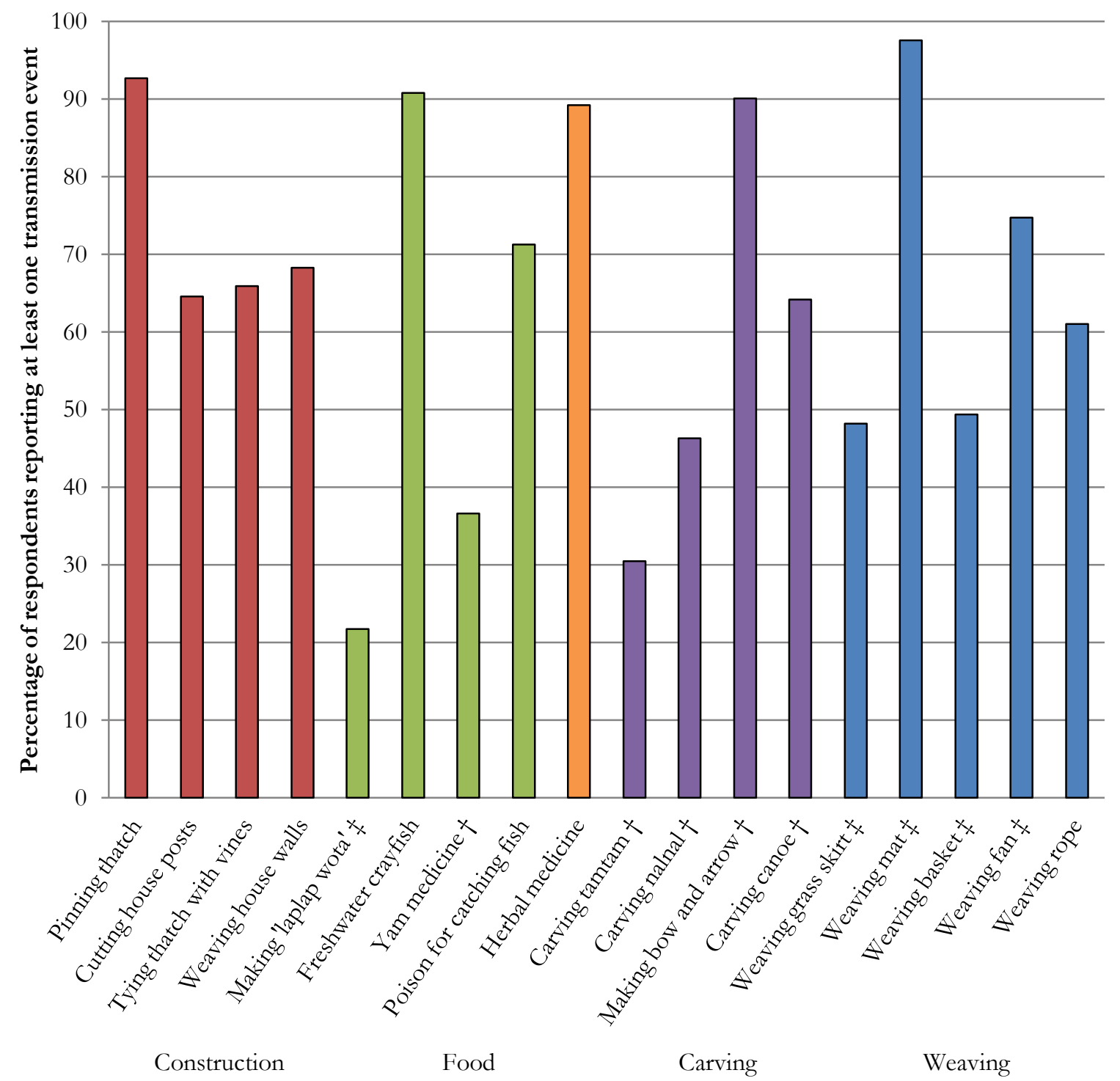

Figure 6.2: Percentage of respondents recording at least one transmission event for 18 ethnobotanical skills. $\dagger=$ Male only skill; $\ddagger=$ female only skill. Percentages of gendered skills calculated from eligible pool only (male $\mathrm{n}=81$, female $\mathrm{n}=83$ )

There were some differences between Unmet and the other communities in terms of percentage of respondents reporting transmission events of the 18 skills. Interviewees at Unmet had a significantly lower percentage of respondents reporting transmission of skills within the domain of food $(U=$ $598.5, \mathrm{p}=.05)$. This was largely due to low figures within the domains of making laplap wota $(6 \%)$ and herbal medicine for enhancing yam growth $(10.5 \%)$, probably because these domains, whilst they were known to participants, were less salient than in the other three focus communities. Participants at Unmet also reported significantly lower percentage of transmission events for the domain of 
carving. This was largely due to low scores in the individual skill of carving nalnal (10.5\%), which participants commented was the job of specialist carvers in the community and not the general populace. This potentially represents a key difference in systems of knowledge transmission between the Big Nambas community at Unmet and the other, Small Nambas, communities (see Section 4.8.2).

Individual-level proficiency score (derived from the number of skills that each participant reported at least one transmission event in) was analysed using multiple regression in the Chapter Five (Section 5.3.2.3). These data showed that proficiency score was positively associated with male gender, age, preferential use of vernacular language, educational attainment, and residence at Dixon Reef (Table 5.18). As such, those participants who cited lower proficiency over all key skills were younger females who prefer to speak Bislama and have had lower levels of schooling.

\subsubsection{Transmission pathway}

Transmission pathways were assessed as vertical, horizontal, oblique, or 'other'. The latter category was used for responses which did not fit the three primary classifications as discussed above. This primarily consisted of learning by observation from unspecified sources, but also included responses such as being taught by dreams (for medicinal plants, total of two responses).

Vertical transmission pathways (cited $49.2 \%$ of the time) were significantly more commonly cited than horizontal $(13.2 \%)$, oblique $(17.5 \%)$, or 'other' (20.2\%) means (nonparametric Friedman's ANOVA, $\chi^{2}(3)=109.18, \mathrm{p}<.001$; post-hoc Wilcoxon tests, applying a Bonferroni correction and thus reporting significance at $\mathrm{p}=0.0167)$. In 13 of the 18 skills, vertical transmission was cited significantly more frequently than other modes of transmission. However some skills differed from this pattern (Table 6.4). ${ }^{24}$ Catching freshwater crayfish, for example, is notable for the high percentage of respondents citing 'other' means of transmission, which was primarily identified as self-teaching through observation of peers and elders. Similarly, knowledge of how to use the fruit of Barringtonia racemosa to poison fish on the reef had a higher proportion of participants citing horizontal transmission: it was commonly noted that these were collaborative activities undertaken with groups of peers, which promote the horizontal transmission of knowledge. Carving tamtam and nalnal had notably high percentages of participants reporting oblique transmission from the elder generation. Interestingly, some participants noted that they had learnt to make nalnal $(n=5)$, bow and arrows $(n=3)$, fans $(n=6)$, and mats $(n=7)$ through the formal school system, when teachers had assigned construction of these items as a task during handicraft sessions. For the skill of canoe carving, there were no significant differences between the four major transmission pathways.

\footnotetext{
24 These data are based on the transmission pathways of only those skills where participants noted that they had experienced at least one transmission event. As such, the sample size for each skill is different
} 
Table 6.4: Percentage of responses per skill per mode of transmission

\begin{tabular}{|c|c|c|c|c|c|c|c|c|c|}
\hline & Skill & $\begin{array}{c}\text { Total } \\
\text { responses }\end{array}$ & Father & Mother & $\begin{array}{c}\text { Both } \\
\text { parents }\end{array}$ & $\begin{array}{c}\text { Total } \\
\text { vertical }\end{array}$ & Horizontal & Oblique & Other \\
\hline \multirow{4}{*}{ 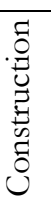 } & Pinning thatch & 148 & 16.2 & 25 & 16.9 & 58.1 & 6.1 & 16.2 & 19.6 \\
\hline & Cutting house posts & 97 & 40.2 & 1 & 8.2 & 49.5 & 10.3 & 12.4 & 27.8 \\
\hline & Tying thatch with vines & 102 & 53.9 & 3.9 & 8.8 & 66.7 & 8.8 & 13.7 & 10.8 \\
\hline & Weaving house walls & 106 & 28.3 & 2.8 & 10.4 & 41.5 & 17.9 & 15.1 & 25.5 \\
\hline \multirow{5}{*}{$\begin{array}{l}7 \\
0 \\
0 \\
\text { I }\end{array}$} & Making 'laplap wota' & 37 & 2.7 & 43.2 & 16.2 & 62.2 & 5.4 & 27 & 5.4 \\
\hline & Freshwater crayfish & 98 & 1 & 10.2 & 8.2 & 19.4 & 10.2 & 3.1 & 67.3 \\
\hline & Yam medicine & 34 & 52.9 & 5.9 & 0 & 58.8 & 5.9 & 32.4 & 2.9 \\
\hline & Poison for catching fish & 107 & 15.9 & 0.9 & 3.7 & 20.6 & 35.5 & 21.5 & 22.4 \\
\hline & Herbal medicine & 144 & 37.5 & 6.3 & 11.8 & 55.6 & 14.6 & 25 & 4.9 \\
\hline \multirow{4}{*}{ • } & Carving tamtam & 24 & 29.2 & 4.2 & 0 & 33.3 & 12.5 & 54.2 & 0 \\
\hline & Carving nalnal & 38 & 31.6 & 2.6 & 5.3 & 39.5 & 5.3 & 42.1 & 10.5 \\
\hline & Making bow and arrow & 77 & 40.3 & 1.3 & 1.3 & 42.9 & 14.3 & 26 & 16.9 \\
\hline & Carving canoe & 53 & 22.6 & 0 & 1.9 & 24.5 & 22.6 & 26.4 & 26.4 \\
\hline \multirow{6}{*}{ 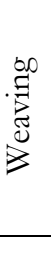 } & Weaving grass skirt & 48 & 2.1 & 43.8 & 12.5 & 58.3 & 0 & 35.4 & 6.3 \\
\hline & Weaving mat & 83 & 1.2 & 63.9 & 12 & 77.1 & 3.6 & 8.4 & 10.8 \\
\hline & Weaving basket & 41 & 0 & 51.2 & 9.8 & 61 & 12.2 & 7.3 & 19.5 \\
\hline & Weaving fan & 61 & 0 & 41 & 13.1 & 54.1 & 6.6 & 8.2 & 31.1 \\
\hline & Weaving rope & 92 & 33.7 & 6.5 & 12 & 52.2 & 9.8 & 20.7 & 17.4 \\
\hline & Median & 80 & 25.45 & 6.1 & 9.3 & 53.15 & 10 & 21.1 & 17.15 \\
\hline
\end{tabular}

There were some limited differences between the most common transmission pathways for men and women. Men cited a significantly higher percentage of oblique transmission than women $(U=$ 2255.0, $\mathrm{p}<.01)$. On the other hand, women cited a significantly higher percentage of responses as 'other' $(U=2690.0, \mathrm{p}<.05)$, the bulk of which was made up of learning through observation with no defined primary teacher. This pattern was also reflected in the individual use domains of construction, food, and medicinal preparation. Within the domain of carving, there was a significant difference between males and females $(U=111.5, \mathrm{p}<.001)$ : all the nine women who reported having learnt this gendered skill learnt through horizontal means from peers.

Within the category of vertical transmission, the same sex parent proved to be the most important transmitter of knowledge (Figure 6.3). Men cited their father as the major transmitter more than women $(U=1698, \mathrm{p}<.001)$, and women cited their mother as primary transmitter more than men $(U=1531, \mathrm{p}<.001)$. There was no significant difference between males and females for citing both parents as equal teachers. 


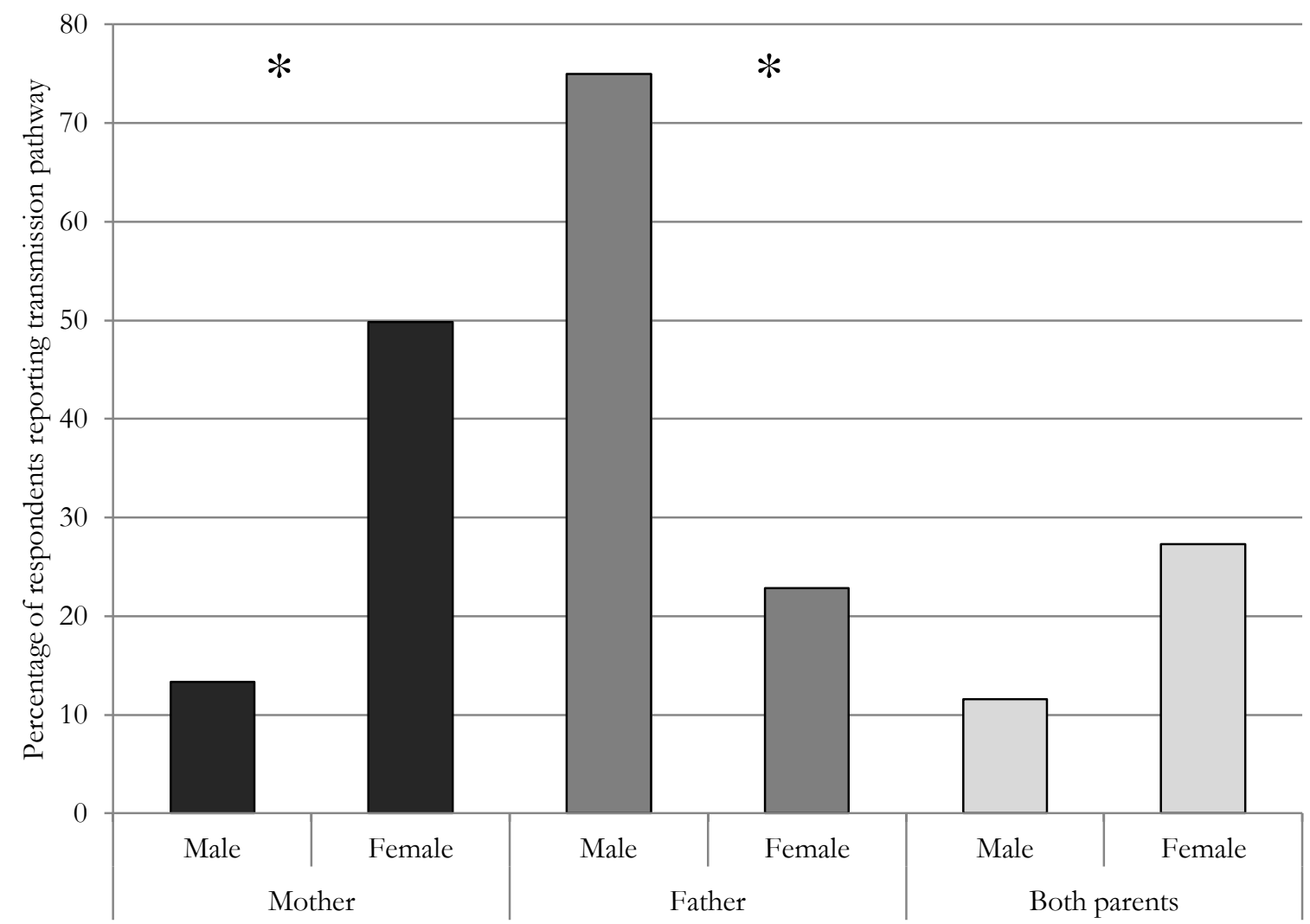

Figure 6.3: Percentage of vertical transmission events for all 18 skills, split via gender of respondents. Percentages calculated from total 'vertical' responses for each gender (male $n=344$, female $n=333)$. * indicates significant difference, $\mathrm{p}<.001$ )

There were no significant differences in the dominant transmission pathway between communities, although participants at Dixon Reef cited a noticeably higher median percentage of participants citing vertical transmission (median at Dixon Reef $=59.2 \%$, all communities median $=53.2 \%$ ), and participants at Lawa reported noticeably lower percentages of oblique transmission (median at Lawa $3.8 \%$, all communities median 21.1\%). Moreover, there was little evidence of differences in the dominant transmission pathway between age cohorts. The exception was within the domain of food preparation and capture, where participants in the youngest age cohort cited a significantly higher percentage of 'other' responses $(U=276.5, \mathrm{p}=.024$, using a Bonferroni correction and reporting significance at 0.025$)$ and a significantly higher percentage of vertical responses $(U=272.0, \mathrm{p}=.018)$.

Individual-level percentage of oblique transmission citations was significantly positively correlated with proficiency and the other measure of TEK from Chapter Five (Table 6.5). In other words, those participants who cited a higher percentage of transmission events occurring from elder experts also scored more highly in measures of TEK. 
Table 6.5: Pearson's correlation of TEK measures with individual percentage of each transmission pathway cited

\begin{tabular}{lcccc}
\hline & Vertical & Horizontal & Oblique & Other \\
\hline Proficiency score & -0.02 & -0.09 & $0.22^{* *}$ & -0.09 \\
Medicinal plant richness & -0.01 & -0.13 & $0.20^{*}$ & -0.07 \\
Ecological knowledge quiz & -0.04 & -0.09 & $0.19^{*}$ & -0.06 \\
Number of fish cited & $-0.17 *$ & -0.02 & $0.22 * *$ & 0.04 \\
\hline
\end{tabular}

\subsubsection{Age of first skill transmission}

The median age of first skill transmission was 15, and ranged between 4 and 54 (Table 6.6). Food preparation skills such as catching freshwater crayfish and using poison to catch reef fish were learnt at the youngest age, with median ages respectively of 10 and 11 . The complex carving skill of canoemaking had the oldest median age of transmission at 18. Interestingly, several domains had small numbers of participants noting an old age of transmission, such as the three interviewees who noted they had been over 50 before they learnt to tie down thatch with vines. This indicates that, in some cases, initial learning of practical skills can occur at any point. Learning of all skills can be a long process and is likely to continue throughout adulthood, especially for complex skills such as the preparation of some medicinal plants.

Table 6.6: Median age of initial skill transmission of 18 key skills

\begin{tabular}{|c|c|c|c|c|}
\hline & Skill & Median (years) & Min & Max \\
\hline \multirow{4}{*}{ 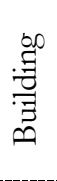 } & Pinning thatch & 14 & 7 & 32 \\
\hline & Cutting house posts & 15 & 7 & 29 \\
\hline & Tying thatch with vines & 15 & 7 & 54 \\
\hline & Weaving house walls & 16.5 & 7 & 37 \\
\hline \multirow{4}{*}{$\begin{array}{l}\bar{Z} \\
8 \\
0 \\
1\end{array}$} & Making 'laplap wota' & 12 & 5 & 47 \\
\hline & Freshwater crayfish & 10 & 5 & 30 \\
\hline & Yam medicine & 15 & 7 & 31 \\
\hline & Poison for catching fish & 11 & 5 & 60 \\
\hline$\stackrel{\oplus}{\Sigma}$ & Herbal medicine & 15 & 4 & 40 \\
\hline \multirow{4}{*}{. } & Carving tamtam & 15 & 6 & 30 \\
\hline & Carving nalnal & 15 & 4 & 40 \\
\hline & Making bow and arrow & 10 & 4 & 40 \\
\hline & Carving canoe & 18 & 9 & 40 \\
\hline \multirow{6}{*}{ 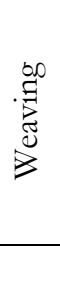 } & Weaving grass skirt & 15 & 5 & 40 \\
\hline & Weaving mat & 15 & 5 & 46 \\
\hline & Weaving basket & 15 & 5 & 43 \\
\hline & Weaving fan & 15 & 5 & 49 \\
\hline & Weaving rope & 15 & 7 & 40 \\
\hline & All skills & 15 & 4 & 54 \\
\hline
\end{tabular}


No significant differences exist among use domains in terms of the age of first knowledge transmission. The only exception is that the first transmission of food-based knowledge occurs at a significantly earlier age (mean $=13.0$ years) than for construction knowledge (mean $=15.6$ years; via Friedman's ANOVA, $\chi^{2}(3)=12.45, \mathrm{p}<.01$; post hoc testing utilising Wilcoxon test $\mathrm{T}=1302, \mathrm{p}=$, $\mathrm{p}<.001)$.

If split into age cohorts, older participants (those aged 60 years and over) reported experiencing transmission events at a significantly earlier age than those in younger age cohorts (between 18 and 30 and between 31 and 60) within the use domains of construction and weaving (respectively, median $=12.75 ; \chi^{2}(2)=8.787, \mathrm{p}<.05 ;$ and median $\left.=12 ; \chi^{2}(2)=7.81, \mathrm{p}<.05\right)$. Participants aged between 18 and 30 reported learning medicinal skills significantly younger than those in the 31 to 60 age cohort $(U=1548.0, \mathrm{p}<.05)$. Via community, in general, age of skill transmission was oldest at Unmet, and youngest at Dixon Reef. Differences were, however, not significant.

\subsubsection{Place of skill transmission}

For most domains the majority of participants explained that there was no specific location for the teaching of specific skills (Table 6.7). Where a location was cited, the house was the most common answer, and this was especially true for skills within the use domains of weaving and medicine. Unsurprisingly, food collection and preparation skills were cited as being learned in the field (including, of course, river and reef environments). The nakamal 25 was rarely cited as an environment for learning TEK skills, and was only cited by the oldest participants. This is due to the fact that nakamal have not been actively utilised (in the traditional sense: see footnote) since the conversion to Christianity of much of the population during the $19^{\text {th }}$ and $20^{\text {th }}$ centuries. At Unmet, the role of special ceremonies, in particular nimangki (grade taking ceremonies), was stressed as environments for learning carving skills of tamtam and nalnal, as these were the only times when the skills were required. As noted, school was cited as the place of learning carving and weaving skills by $4.3 \%$ and $4.9 \%$ respectively, and participants commented that occasionally teachers would include such lessons in handicrafts classes.

\footnotetext{
25 The meaning of the term nakamal has shifted towards 'meeting house' or 'kava bar' in recent years, and it remains in common usage in these senses (McCarter, unpublished field notes). However, it originally referred to the men's club-house in pre-contact village life, in which access was restricted to men only and which formed the basis of social and ritual life (MacClancy, 2002). It was in this location that men's knowledge would primarily have been transmitted. Nakamal were often disbanded following the conversion of the community.
} 
Table 6.7: Major location for teaching TEK skills, per domain (percentage of total responses)

\begin{tabular}{lcccccc}
\hline & Building & Food & Medicine & Carving & Weaving & Total \\
\hline No specific location & 85.7 & 79.2 & 74.3 & 74.0 & 67.1 & $\mathbf{7 6 . 1}$ \\
Field & 1.2 & 15.3 & 4.2 & 1.7 & 0.2 & $\mathbf{4 . 5}$ \\
Nakamal & 0.0 & 2.1 & 0.7 & 6.5 & 1.1 & $\mathbf{2 . 1}$ \\
Home & 12.0 & 2.3 & 19.4 & 5.0 & 26.4 & $\mathbf{1 3 . 0}$ \\
School & 0.9 & 0.5 & 1.4 & 4.3 & 4.9 & $\mathbf{2 . 4}$ \\
Special ceremony & 0.3 & 0.7 & 0.0 & 8.6 & 0.2 & $\mathbf{2 . 0}$ \\
\hline
\end{tabular}

There was no significant variation of major place of skill transmission between communities, age cohorts, or genders. However, analysis via gender and community reveals that only elder males at Unmet report learning skills within the nakamal environment (Figure 6.4). One interpretation of this result is the finding demonstrates the shift from the nakamal (associated with the interior) environment, which may be only now remembered by older men at Unmet (the most recently established community). It is worth noting that the nakamal would have been a male-only environment, so it is not surprising that no women (even elders) report learning there.

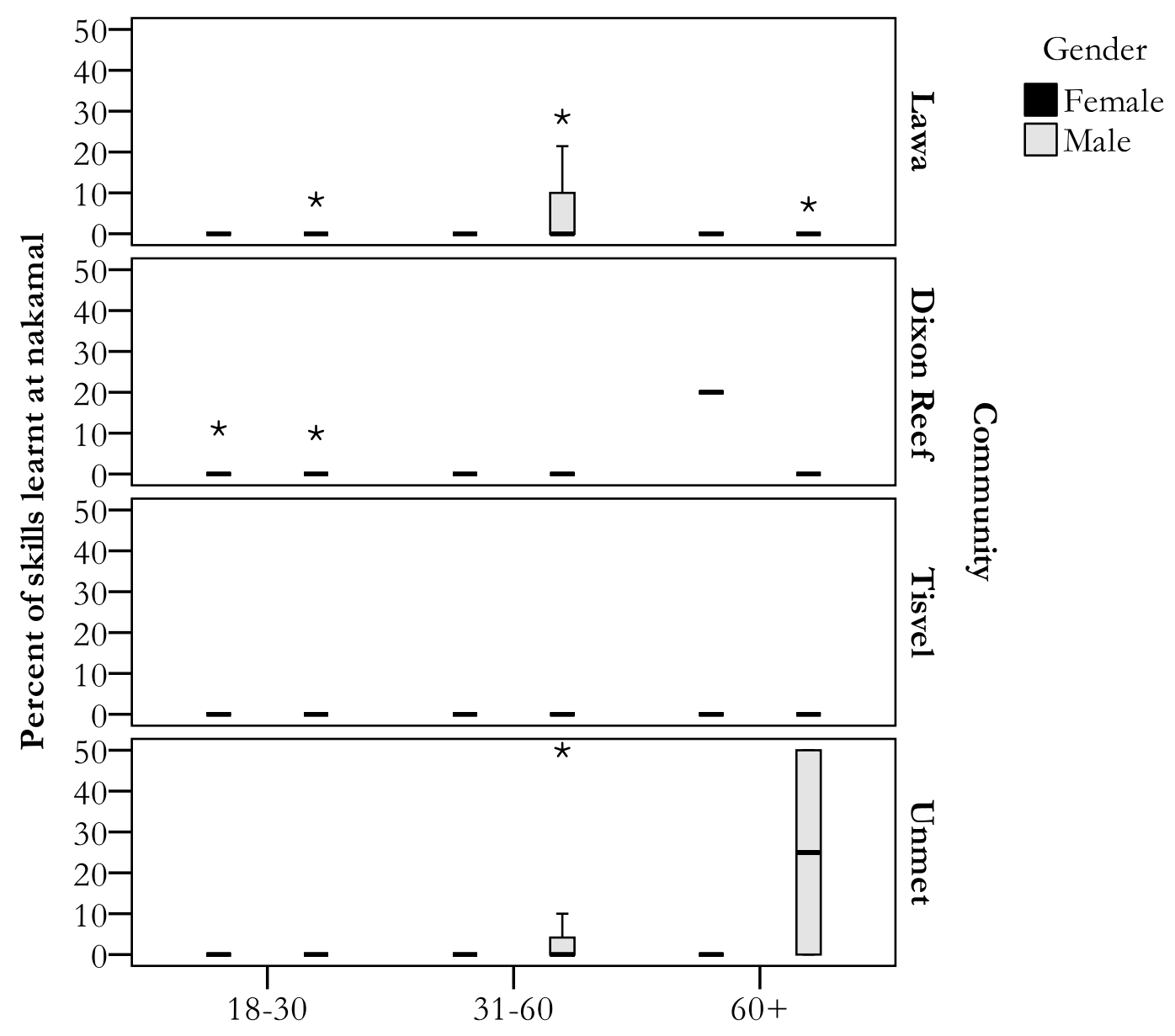

Age cohort

Figure 6.4: Major place of learning key skills, by community and gender. $*=$ outlier 


\subsubsection{Perceptions of changes in TEK transmission (semi-structured interviews)}

This section reports the results of the semi-structured interviews with 18 expert informants.

Participants were unanimous in declaring that there had been large changes in transmission over recent years, thus supporting data from the scoping study in 2008 (Section 4.9). These changes were usually expressed to be consequences of, or concurrent with, social changes which have accompanied the introduction of formal schooling, western markets, and the Bislama language. Table 6.8 summarises the factors which were linked by participants to intergenerational changes in transmission of TEK knowledge and skills, categorised by developmental niche component (Super and Harkness 1986; Super and Harkness 2002). I observe that these factors are interlinked, and represent a mix of ultimate and proximate issues: for example, decreased participation in ceremonial activities may have been a consequence of geographic shifts, because ceremonies were discouraged by the church establishment. 
Table 6.8: Summary of perceived intergenerational changes in transmission of TEK knowledge and skills, categorised into the developmental niche components

Developmental Niche components and related themes $\begin{gathered}\text { Number of interviewees } \\ \text { identifying theme (total } n=18)\end{gathered}$

\section{Environmental}

Changing setting for TEK transmission

Geographic shifts from interior to coastal environment 3

$\begin{array}{ll}\text { Increased formal schooling } & 14\end{array}$

Decreased time at nakamal/home environments 5

Decreased participation in ceremonial activities 6

Death of elderly community members 8

\section{Social-cultural}

Changes in the strategies for TEK transmission

Vernacular mnemonics for recalling illnesses and diseases 2

$\begin{array}{ll}\text { Increased respect for written texts } & 15\end{array}$

Decreased respect for oral histories 3

$\begin{array}{lr}\text { Decreased ability to recall orally transmitted knowledge } & 10\end{array}$

Lack of designated place for TEK transmission $\quad 12$

Decreased use of sand-drawing for kastom stories 3

Introduction of new techniques from peers 4

$\begin{array}{ll}\text { Linguistic erosion within community } & 16\end{array}$

\section{Individual}

Transmitters

Decreased ability for parents to teach 9

$\begin{array}{ll}\text { Lack of effort from parents } & 8\end{array}$

Elders requiring cash payments 3

Learners

Lack of respect for traditional norms (payments to teachers) 5

Lack of ability to keep secret information safe 3

Lack of identity/ties to community; closer affiliation to

'whiteman' ways 7

All the themes in Table 6.8 describe important intergenerational shifts in the socio-cultural environment for TEK transmission within the focus communities on Malekula. Some of the themes were more commonly cited than others, especially the role that linguistic change is playing in interrupting the transmission of TEK. Interviewees noted the critical importance of language in discussion of all aspects of kastom, especially those necessitating detailed environmental awareness such as ethnomedical knowledge. This does not simply extend to the naming of plants, but also the ability to recognise illnesses. Indeed, the two facets are linked: one healer at Dixon Reef noted that the names of plants were associated with the names of illnesses, and consequently linguistic proficiency was intrinsically linked to ability to heal. Because most of the younger generation are perceived not to have the detailed plant vocabulary, this impacts on their ability to heal, and thus adequately acquire or disburse knowledge. 
Most of the themes in Table 6.8 coalesce into two key nodes of socio-cultural change in knowledge transmission: change in the setting of TEK transmission, and changes in the strategies for TEK transmission. I discuss these two themes in detail below.

\subsubsection{Changes in the setting of knowledge transmission}

Participants were usually quick to note that the structured and gender-separated environment of knowledge transmission was now no longer a feature of village life. This was commonly expressed by noting that the children were more 'free' these days, and that they were not beholden to old restrictions on movement or conversation. This was noted by one female teacher: “... before, our grandparents were stuck at the nakamal, and the kids could not run about everywhere. Today, it is not the same, and the kids run about free all over the place" (Female, age 45). Therefore children are perceived to have less respect than their forebears for the importance of TEK knowledge and skills, and less interest in acquiring knowledge in the manner it was passed on in the past. The graded rank system has not been operative for over a generation in most areas, and whilst most boys still pass through circumcision around puberty this is no longer associated with a shift in the mode of knowledge acquisition. Moreover, the informal setting for the transmission of knowledge was no longer perceived to be effective: “...it is like this, knowledge is being lost, it is not being passed on. Before, every afternoon, the children would gather and there would be kastom stories. The storytellers would tell them all the time, and the kids would ask questions. But now, there is no time for that sort of thing, it does not happen anymore" (Male, 68). The increased freedom available to children and learners had meant that there was typically less attention given to social norms, respect, and familial boundaries: “. . kastom before was a system of building boundaries between the different families; and there were never people who wanted to break down those boundaries" (Male, 52).

One elder male noted that he actively withheld information from pupils these days, because he perceived that the systems were no longer in place to ensure correct use of secret or dangerous knowledge. In general, this loss of structure and removal of the context for the transmission of knowledge was perceived to be behind a reticence on the part of some elders to transmit their TEK knowledge and skills. This was as much about the lack of a dedicated space as anything: “...these things are being lost because now we are not thinking for the future. Because there is no longer a place to tell these things, if you sit with the elders, you have to ask. If you do not ask them to tell you, they cannot pass their knowledge to you" (Male, 52). A critical part of the setting for knowledge transmission in the past was structured for the appropriate payment and respect for knowledge holders. Elders were often unwilling to pass on information unless the correct payment and respect had been recognised: “...In terms of the rights to this knowledge, they do not want to pass it on because people have not paid enough for it. You bave to pay for this 
knowledge..." (Male, 30). Moreover, participants noted that because the systems of payment associated with graded rank (such as pigs and yams) were now not valued as highly, cash had become the most valued item for reimbursement. Indeed, participants indicated that elders may refuse to transmit TEK without significant cash payments: "... before, the teacher would accept payments of mats, or yams. But now, if you want to make a tamtam [slit drum] like this one here, they think about money only..." (Male, 49).

Participants pointed to the role that formal schooling has played in shifting the context of knowledge transmission, noting that schooling takes up significant amounts of the children's time and passes on large amounts of competing information. As such, participants perceived that TEK knowledge and skills now vie for time and space alongside the requirements of learning language, maths, and other staples of school life. Moreover, teachers and authority figures are in unique positions to dispense large amounts of information that parents within the village do not have access to, thereby changing the nature and transmission pathway of knowledge. For example as one elder male informant noted that "...today, I can be sitting with the children and trying to teach them medicine, and telling them about a special one...then they go to school, they study all sorts of other things... and their brain is too small, everything else is lost" (Male, 52.).

Finally, the introduction of new TEK skills and techniques from other areas was perceived to have eroded the need for rigidly transmitted vertical knowledge. Technologies and techniques, such as the use of diving equipment for catching freshwater crayfish or for building canoes, now spread rapidly amongst networks of friends and contacts. As a consequence of being more 'free', knowledge was perceived to spread more easily throughout the social network, thus removing the emphasis on traditional transmission mechanisms: "Kastom knowledge changes following the thinking of people....there is one technique where someone can learn again, but if it is lost then people will just learn new one from somewhere else, like. the way we use nets or guns rather than the way these things were done before... it is not so much our tradition now, but is a new technique that belongs to anotherpeople... and it often means you can catch more every time, so people want to learn it from us..." (Female, 29).

\subsubsection{Changes in strategies of knowledge transmission and storage}

Participants perceived that the key dynamic in terms of the strategies for the storage and transmission of knowledge was the shift from oral to written methods. There was a common concern that memory within the community was now no longer sufficient to recall most of the kastom stories and histories which encoded knowledge and practice before. This was observed by one elder church official: “... sometimes, the elders cannot take the things from before and make them permanent... for example, our histories and kastom stories, they are really important, but now us ni-V anuatu are taking the thinking of the whiteman, which means we do not have memory for them anymore, they cannot stay in the head for a long time. 
Then, when the elders die, the stories and histories do too, they cannot go to the next generation" (Male, 52).

Knowledge that was encoded in books (which is commonly religious or school-based) was perceived to have a greater permanence and an increased ability to be able to stand in competition with other forms of knowing. As such, most participants foresaw that the transmission of TEK knowledge and skills needed to be based more around written texts and exercises. As one young female teacher wryly noted: "... if it is not written down these days, it is not really respected" (Female, 32).

Not all participants were comfortable with the shift from oral to written transmission. Some noted that this would intrinsically change the nature of the knowledge itself, as one young fieldworker observed: “...I can write TEK knowledge down, but it is a big change... the kids want to write everything down. But I have some special, secret things that people are not going to want to be written down, because then everyone will be able to see them" (Male, 30). In terms of knowledge transmission, transcribing knowledge that was previously oral places power in the hands of those who are able to record the knowledge, who are most commonly western researchers. This may, in turn, influence the domains of knowledge that were written down. Moreover, participants noted that such processes favour the power structures that are dominant in any one community, such as the privileging of one language over another: "Yes, I can write all of these things down. But there is no one who knows it perfectly like before, and there are some kastom medicines that I do not know, and that I cannot write down. But another thing is that in Novol, there are some medicines, and I should write these down. But the other languages here, they bave different medicines too..." (Male, 49).

Tensions around the changing nature of knowledge and knowledge transmission are also themes of Chapters Seven and Eight.

\subsection{Discussion}

The quantitative and qualitative data presented here provide a snapshot of the cultural transmission of TEK knowledge and skills on Malekula. As a first observation, I note that most of the TEK skills surveyed are clearly still being actively transmitted within the study communities. In response to all the skills tested, and throughout broader discussions of TEK, participants would refer to the value of TEK in modern life. This corroborates evidence from Vanuatu that traditional culture and kastom still play a central role in contemporary society (e.g. Hickey 2003b; Regenvanu 2005)

\subsubsection{Transmission pathways on Malekula}

Vertical transmission was the dominant mode of cultural transmission for the sampled TEK knowledge and skills. This corroborates the bulk of research of this kind within ethnobiology, which typically note that parental figures are critical for the transmission of knowledge (Casagrande 2002; 
Eyssartier et al. 2008; Hewlett and Cavalli-Sforza 1986; Lozada et al. 2006; Ohmagari and Berkes 1997). The role of same-sex parent (mothers for females, and fathers for males) seems to be especially important, in a finding which also echoes other research (Martinez-Rodriguez 2001; ReyesGarcía et al. 2009). This is consistent with the strong gender divisions present in ni-Vanuatu society (Bolton 2003) and the gender-specific nature of ten of the 18 key skills tested.

Previous studies of the cultural transmission of TEK have found that between $50 \%$ and $80 \%$ of skills are transmitted vertically (Casagrande 2002; Eyssartier et al. 2008; Hewlett and Cavalli-Sforza 1986; Ohmagari and Berkes 1997). The findings presented here largely corroborate these trends in noting that vertical transmission was dominant in all but five of the skills surveyed, and accounted for $49 \%$ of transmission pathways cited. In the global studies noted above, as on Malekula, vertical transmission was the dominant mode through which most TEK had been transmitted in the past. This is not particularly surprising, given the attachment and contact between parents and children throughout the early life phases. On Malekula, this may have particularly important given the secretive and structured nature of domains of TEK such as ethnomedical knowledge. However, oblique, horizontal, and 'other' means of transmission were important for the transmission of some skills. For food gathering skills, for example, horizontal and 'other' modes of cultural transmission was the norm, and participants commonly noted that they had acquired these skills in the communal setting of the food gathering environment. This corroborates data from other studies: for example, in the Western Ghats in India, Cruz-García (2006), observed that children gain experience and skills in the gathering of wild food plants on the way to and from school, which accounted for a significant percentage of the knowledge acquisition by pupils in the study.

Data from the structured interviews indicate that the 18 skills surveyed were first transmitted between the ages of 10 (catching freshwater crayfish) and 18 (carving canoes). This is in line with findings from other research, who have noted that foraging and food preparation tasks are acquired at an early age, with more complex craft-based tasks at a later age (Hewlett and Cavalli-Sforza 1986; Lozada et al. 2006; Ohmagari and Berkes 1997; Ruddle and Chesterfield 1977). It is also broadly consistent with accounts that stress that while theoretical ethnobiological knowledge may be acquired at a very early age (between three and ten years), practical skill and ability takes longer to build up (Godoy et al. 2009; Hunn 2002; Zarger 2002). The structured interview data also indicated that there was no fixed place for the transmission of TEK within the focus communities. Most notably, the percentage of respondents citing any TEK transmission within the nakamal system (which was noted to be the fundamental place of teaching pre-contact) was very small, and limited to only those participants who had been raised before communities shifted to the coast. 
The quantitative data, then, broadly corroborate other studies and provide valuable information on the structure of the cultural transmission of TEK on Malekula. These data are important, as a lack of ethnobiological studies of cultural transmission hampers our ability to understand the acquisition and dispersal of knowledge in rural communities (Zarger 2002). Moreover, they serve to contextualise the data gleaned in Chapter Five (a theme I return to below), and represent the first study of this kind to be undertaken in the Pacific. Both streams of data, however, also provided evidence that these systems for the cultural transmission of TEK are changing.

\subsubsection{Changes in cultural transmission on Malekula}

Evidence for changes in systems of cultural transmission on Malekula can primarily be drawn from the results of the semi-structured interviews. These data indicate that interviewees perceive there to have been major changes in both the setting and the methods of cultural TEK transmission on Malekula. The DN framework used above identifies a variety of proximate and ultimate of environmental, socio-cultural, and individual factors which are driving these changes in transmission. Importantly, several of these (for example, 'lack of time', 'lack of respect for oral histories', 'closer affiliation with whiteman ways') were perceived to be driven by the formal school system. In particular, participants commented on the ways in which the increased importance of written materials had impacted on knowledge transmission within the communities, a theme that is common throughout Chapters Six and Seven. Interviewees indicated that children were now unable to learn theoretical TEK knowledge without writing down significant portions it. This was perceived to impact on their practical ability: for example, it was often commented that children cannot prepare medicines, as they do not understand the vernacular names of the plants. In this sense, the increased importance of written culture has created a disjunct, which did not exist before between theoretical and practical knowledge.

Changing strategies for knowledge transmission raise a host of other issues as well. Because written materials are more easily shared and difficult to protect, they more easily support horizontal forms of TEK transmission. This, in turn, creates issues of power and control over information. Indeed, the interview comments which acknowledged that some community members are now refusing to dispense information because the younger generation were not sufficiently respectful are worrying, as they indicate the presence of feedback loops. These loops may increase the attenuation of TEK transmission systems: as the younger generation become more integrated into the western modes of thinking (potentially promoted by the school system), so the ability of the elders to transmit TEK in the appropriate setting, using the appropriate methods, decreases. In this sense, the loss of the physical space for TEK transmission (such as the nakamal or the village environment) has also resulted in a loss of cognitive space in which pupils and resource people can interact (cf. Cristancho and Vining 
2009). The shift in the context and space of TEK transmission forms one of the key nodes of debate within the community around the place of kastom and tradition in modern life. The next two chapters deal explicitly in the ways in which this debate is being negotiated, through the formal school system and through bottom-up 'kastom schools' for the revitalisation of TEK.

Do the quantitative data support the suggestion of significant recent changes in cultural transmission suggested by the qualitative data? As I have noted in previous chapters (see Section 2.4.1 or Section 5.4.2), it is difficult to use synchronically-collected quantitative data to draw evidence of diachronic changes in TEK systems (Godoy et al. 2009). However, we may be able to hypothesise that, if the changes in systems of cultural transmission have been as dramatic as described by the expert participants, we might be able to see significant differences between the age cohorts of the participants. This is because the systems of cultural transmission that would have been in place while older participants (aged $60+$ ) were learning may have been different to those in place for younger age cohorts (aged 18-30 and 31-60), who grew up in the contemporary social environment.

There was limited evidence of changing patterns of TEK transmission on Malekula from the quantitative data. Data on transmission pathway, for example, were marginally patterned by demographic variables, and only within the use domain of 'food' were any significant differences between age cohorts evident. It is also important that, in Chapter Five, age was significantly associated with overall proficiency score (Table 5.17), as it indicates that older participants indicated transmission events for more of the skills overall than younger participants. Moreover, proficiency score was associated with indicators of modernisation including linguistic preference, strengthening the hypothesis that overall skill transmission is receding amongst current generations. However, support from the quantitative data for changing systems of TEK transmission was fairly equivocal, and there are no strong patterns in the data.

The lack of conclusive patterns evident in the quantitative data may not be surprising. It is likely that the major intergenerational interruptions in TEK transmission referred to during the semi-structured interviews closely followed the major cultural and ecological shifts (respectively, from kastom to the church and from the interior to the coast) that characterised the period between 1920 and 1970. This hypothesis has been supported in other contexts: for example, work amongst the Zinacantec Maya in Chiapas has shown that groups rapidly adjust transmission strategies in times of social and cultural change (Greenfield et al. 2000). Such results are mirrored by Casagrande's (2002) findings that Tzeltal Mayan men rely heavily on horizontal transmission events in new and non-stable environments, where transmission of ethnomedical knowledge is overwhelmingly vertical in their home environment. As noted by Greenfield et al. (2000): “...changes in the processes of cultural apprenticeship produced a new generation that was well adapted to the changed ecological 
niche...there was a tight relationship between the changing ecological niche and a changing developmental niche" (p 372). In short, because most of the interviewees were born after these major changes had taken place, these changes are no longer visible through the quantitative data. This hypothesis is supported by Figure 6.4, which shows that only elder males at Unmet report learning skills in the nakamal environment. Because Unmet is the most recently established community (in around 1972), it appears that of all the interviewee sub-groupings, they were the only ones have experienced the 'traditional' environment for skill transmission. It is notable, however, that this did not result in increased proficiency in participants at Unmet, at least amongst the 18 sampled skills.

Some of the tested skills were clearly less salient than others. Skills associated with ceremonial occasions, or with particular forms of food preparation, had low percentages of respondents reporting transmission events (Figure 6.2). Interestingly, the percentage of transmission that participants cited as having come from oblique sources was positively associated with several measures of TEK (Table 6.5). These correlations are important, as they indicate that those participants who had greater contact with elder experts are more likely to have greater exposure to a range of skills, and may have greater overall ecological knowledge. This indicates that one strategy to encourage the maintenance of knowledge and skills, in particular the carving of tamtam and nalnal, might be to facilitate contact between younger age cohorts and elder resource people.

The fact that the majority of participants did not report transmission events for some skills could be taken as evidence that some skills are not being actively transmitted. Indeed, previous research has speculated that such patterns indicate that these skills are at risk of disappearance (Lee et al. 2001; Ohmagari and Berkes 1997). The hypothesis that younger interviewees have less proficiency in TEK skills is supported, again, by Table 5.18, which shows that in general younger participants have lower overall proficiency at the tested scores than others. I note that two factors prevent me from drawing strong conclusions in this regard: first, the presence of a transmission event is not, in itself, a good indicator of the complete transmission of the skill or mastery of the skill by the participant. Objective assessment of proficiency would require in-depth participant observation and testing which was outside the scope of this research. Second, complex and valued skills such as carving tamtam are likely to be only passed on with increasing age. Therefore, surveys such as this one that do not have a longitudinal component (i.e. a series of objective measurements over time) will be limited in their ability to discern skill loss.

Interestingly, within the use domains of carving and weaving, participants in the oldest age cohorts reported learning skills significantly earlier than those in the youngest age cohorts. This may indicate that that knowledge acquisition is occurring later in life than in previous generations, and that this is 
indicative of an intergenerational shift in the ways in which TEK skills in these domains is transmitted. This interpretation has some support from the qualitative data, where interviewees often noted that the lack of structure, and the presence of formal schooling, meant that primary-age pupils did not have the chance acquire knowledge and skills as before. Other work has noted similar patterns: for example, in communities of James Bay Cree women, Ohmagari and Berkes (1997) found that the introduction of formal schooling had slowed both the acquisition of skills. Moreover, they found that it had also lowered the overall level of skill mastery: "...the 'learning' of the skills... was different from that in older generations... [elders] had learned the skills fully by their midteens... by contrast, most of the girls and young women were nowhere near that stage" ( $p$ 210). The delayed learning of skills may impact on later ability to learn and acquire knowledge, including potentially causing 'acquisition deprivation', if knowledge and skills are not gained during a critical period in childhood and early teenage years (Hunn 2002). As such, the delaying of skills acquisition may be one of the mechanisms underlying the lower proficiency in younger participants and the lack of transmission events for several of the 18 skills.

\subsubsection{TEK transmission, TEK variation, and mixed methods approaches}

Some of the findings presented in Chapter Five indicated that ethnomedical knowledge was homogenising amongst younger age cohorts, and that decreases in the richness and scope of TEK was associated with linguistic shift, market visitation, and, in some cases, formal education. Because the surveyed TEK is transmitted within systems of cultural transmission, we might expect that corroborating patterns will be visible within the data in this chapter.

Certainly, the qualitative data appears to support the trends outlined in Chapter Five. In focussing on the loss of context and methods for the transmission of knowledge, participants identified a mechanism for the homogenisation of medicinal knowledge. That is, these data indicate that the elder generation no longer have the capacity (both in terms of the physical space and the means of communication) in which to transmit idiosyncratic or unique ethnobotanical knowledge of the interior bush environment. Moreover, some the important variables that were noted to pattern knowledge in Chapter Five (in particular linguistic preference) were also explicitly mentioned by interviewees as being responsible for interrupting systems of cultural transmission. The quantitative data also offer some support in indicating mechanisms for TEK erosion. In particular, the positive correlations observed between percentage of citations from oblique sources and proficiency support the suggestion that interruptions to cultural transmission alter the developmental niche for TEK, and thus impact on the patterning of individual-level knowledge (Greenfield et al. 2000). This may impact on the saliency for key TEK skills and ultimately result in the erosion of knowledge and practice. 
That said, these data also offer ample evidence of the dynamism of TEK systems. This is perhaps best illustrated by the example of canoe carving amongst the four focus communities, which was the only domain in which there was no significant difference between pathways for knowledge transmission (i.e. there was an equal chance of transmission via vertical, horizontal, oblique, or 'other' means). Moreover, the initial transmission event for the carving of canoes occurred at significantly later age (median of 18) than for other skills (median of 15). In both characteristics, canoe-carving differed from the other 17 skills. This likely reflects the geographic shifts which appear that have influenced TEK of the marine environment (Chapter Five). None of the four focus communities consider themselves to be 'man solwota' (people of the ocean), and participants in each location continue to hold traditional lands in the interior. Before the shift to the mission to the coast, interior dwelling peoples would have traded with coastal people for essential goods, and did not use canoes (Huffman 1996). As such, canoe carving is a relatively recent addition to the corpus of 'traditional', kastom, knowledge. This knowledge, can, of course, build up relatively quickly: Dixon Reef, which had the highest percentage of transmission events of canoe-cutting between the four communities, was only established in approximately 1950 (Lynch and Crowley 2001). Importantly, it is possible that knowledge of canoe carving was never bound inside rigid structures of vertical transmission: having been brought in from outside, it is now taught and acquired from a range of sources (as with the skill of crossbow hunting amongst the Aka Pygmies in Hewlett and Cavelli-Sforza (1986)).

\subsection{Conclusions}

This research used a mixed methods approach to describe the cultural transmission of TEK in four communities on Malekula. The data indicate that the cultural transmission of the 18 skills is largely vertical, is not conducted at an assigned place, and usually begins during early teenage years.

Pathways and strategies for knowledge transmission do, however, differ between different domains of knowledge. I note that the qualitative data strongly indicate that cultural transmission of TEK is changing on Malekula, and that some of the quantitative data serve to corroborate these trends. Data on cultural transmission is a useful means of discussing the process of cultural change, and contributes to our ability to explain the observed variation in TEK in the previous chapter.

The data presented in this chapter make three important contributions to the literature. First, they extend our knowledge of the ways in which TEK is transmitted between actors within social networks in rural communities. The finding that most transmission is vertical is consistent with some international literature (e.g. Eyssartier et al. 2008), and indicate that some domains of TEK should be expected to be relatively conservative and slow to include change and innovation (Hewlett and Cavalli-Sforza 1986). However, the mode of cultural transmission is variable between domains of knowledge, with elder experts playing a critical role in the transmission of ceremonial and rarely used 
knowledge. This confirms that reconnecting transmission pathways between elders and youth may be critical for supporting the transmission of some knowledge domains (cf. Florey 2009), especially as interview comments indicated that many parents did not have sufficient knowledge to transmit vertically.

Second, in explicitly describing changes in the cultural transmission of TEK, this chapter identifies a mechanism that may underlie the patterns of TEK homogenisation and dynamism observed in the previous chapter. This is a novel contribution - although some have looked at changes in systems of cultural transmission (e.g. Cristancho and Vining 2009), such observations have not been linked to quantitative data on the variation or cultural transmission of TEK. This is a critical piece of the puzzle, as the persistence and erosion of TEK is governed by processes of cultural transmission, and our understanding of the potential for TEK to erode will be incomplete without delineating how systems of cultural transmission might be changing (Zarger and Stepp 2004). Importantly, the changes highlighted in the setting and strategy for knowledge transmission, as identified by the 18 TEK experts, have potential to facilitate the dynamic patterns observed in Chapter Five. For example, loss of vernacular mnemonics for recalling diseases may have facilitated the homogenisation or loss of the ethnomedical knowledge outlined in Section 5.2.3, however the increased time in formal school may result in an increases sharing of knowledge of the marine environment, thus explaining the results outlined in Section 5.3.2.1.

Third, this chapter shows the value of mixed methods approaches for discussing complex processes such as changes in cultural transmission. The quantitative and qualitative methods presented in this chapter serve to complement each other, and effectively answer different questions surrounding the nature and changes in cultural transmission of TEK. Quantitative data, although useful for gathering rapid and comparable data with which to assess the profile of cultural transmission of TEK do not have the explanatory power of in-depth ethnographic accounts (Zent and Maffi 2009). Moreover, as noted, quantitative methods are extremely limited in their ability to detect diachronic change, a deficiency which the semi-structured interviews are better able to address. This was assisted by the use of the DN framework, a relatively novel approach within ethnobiology (Cristancho and Vining 2009).

Finally, I observe that these findings indicate that both the setting and the strategy for cultural transmission are critical nodes of change and can influence the persistence or erosion of traditional knowledge. As such, approaches seeking to maintain and revitalise TEK should pay specific attention to both aspects of transmission, as different approaches (such as top down and bottom up methods) will differ in their impacts on the setting and strategies for knowledge conservation (Bates 2009; Florey 2009). Moreover, changes in setting and strategy for knowledge transmission may influence 
different domains of knowledge in distinct ways, and may need to be adapted to specific, community-identified, and at-risk facets of TEK (Zent 1999). Over the next two chapters, I examine these issues in more depth, and discuss how both formal school and local educational modules may be able to maintain TEK and address some of the issues raised here and in Chapter Two. 


\section{The value of TEK to formal school in}

Vanuatu

In Chapters Five and Six I have focussed on discussing processes and patterns of TEK variation and change. They have shown that it is likely that systems of TEK on Malekula are at risk, and have presented evidence indicating individual and community-level variation of TEK, as well as major changes in systems of cultural transmission of TEK. Given that TEK is an immensely valuable resource, how might we work towards ensuring the maintenance and revitalisation of this knowledge? What might be some of the key tools, and what are some of the key advantages and disadvantages to these approaches? The following two chapters begin to address these questions, by examining both top-down (this chapter) and bottom-up (Chapter Eight) approaches to TEK maintenance. This chapter addresses objective 3(a), which was to 'assess perceptions of top-down measures for the maintenance and revitalisation of TEK, by assessing the value of TEK to formal school curricula.'

The following chapter has recently been published in the Journal of Ethnobiology and Ethnomedicine (McCarter and Gavin 2011). Please note that this article has been slightly altered from its published form: I have removed the 'Setting' section, which has already been described in Chapter Four above; have made some minor editorial changes to improve its fit with the thesis; and have changed 'we' to 'I' throughout. The latter change is necessary for the thesis because I was solely responsible for drafting the manuscript, despite the presence of Michael Gavin as a co-author in the published version.

\subsection{Background}

The spread of western modes of formal education (defined here as institutionalised, chronologically graded, and hierarchically structured systems of education (La Belle 1982)) has been recognised as a 
key driver of global social change (Dewey 1937). There are serious concerns, however, that formal education systems in some areas of the world do not adequately account for local knowledge and cultural diversity (Brayboy and Castagno 2009; Ruiz-Mallen et al. 2010). This results in school systems that are ineffective in attaining educational outcomes and which may actively erode cultural and linguistic diversity (Botha 2010). In consequence, there have been repeated calls over the last decade that local content should be included in education curricula (Aikenhead 2001; Brayboy and Castagno 2009; Ruiz-Mallen et al. 2010). In particular, the inclusion of traditional ecological knowledge (TEK) into formal schooling has been advocated by a variety of commentators (e.g. Heckler 2002; Zent 1999; Zent and Zent 2004).

Commentators have advanced four principal arguments for the inclusion of TEK in formal education systems. First, such approaches may contribute to the maintenance and revitalisation of TEK (Zent 1999). Recent quantitative work has shown that TEK can erode under the influence of various factors associated with modernisation, including integration into the cash economy (Godoy et al. 1998; Godoy et al. 2009; Reyes-García et al. 2005) and linguistic erosion (Benz et al. 2000; Zent 2001), as well as formal education (Cruz García 2006; Voeks and Leony 2004; Zent 1999).

Proponents have argued that the introduction of TEK at pre-school, primary, and secondary levels may increase rates of intergenerational knowledge transmission, in part by legitimising TEK for younger generations and giving it the same status as western knowledge (Kimmerer 2002). This arrangement would leverage existing educational infrastructure, thus avoiding the need for costly novel interventions for TEK conservation (Reyes-García et al. 2005). In addition, interventions of this type would have the advantage of working through the predominant existing drivers of cultural change rather than against them, thus potentially mitigating the adverse effects of contemporary education models on TEK (Zent and Zent 2004).

Second, a growing body of evidence suggests that the inclusion of TEK in formal school curricula might be an important means of increasing student's awareness and participation in environmental issues (Ruiz-Mallen et al. 2009; Taylor and Mulhall 2001). Most environmental knowledge is acquired at an early age (before 12) through sustained contact with the natural world (Bates 2009), tutelage by parents (Hewlett and Cavalli-Sforza 1986; Ohmagari and Berkes 1997), or play with peers (Hunn 2002; Lancy 1996; Zarger 2002). Such experiences have a fundamental impact on individual cognition and ability to acquire local environmental knowledge and skills (Zarger 2002). Formal education systems often remove children from this learning context at an early age, which can result in 'acquisition deprivation' and may inhibit a student's capacity to acquire environmental knowledge (Ruiz-Mallen et al. 2009). The use of TEK in school curricula may assist in mitigating impacts such as this. 
Third, introducing local content such as TEK may contextualise formal education systems, making them more relevant and providing a better sense of place and identity to pupils (Castagno and Brayboy 2008). This would address key criticisms of the classroom centred, exam based nature of western-style formal education, which can contrast with indigenous systems of cultural transmission (Thaman 2000). Studies have noted that contextualised education systems that use locally relevant information can enhance understanding of curricular knowledge, in part through linking the theoretical knowledge of the classroom with practical, lived, reality (Castagno and Brayboy 2008; Reyes-García et al. 2010). Moreover, contextualised education systems can increase the acquisition of knowledge by empowering students, reinforcing learner self-esteem, and maintaining individual and collective cultural identity (Lipka 1994; UNICEF 2003). Similar approaches have received support at an institutional level, most notably from a keystone UNESCO document known as the Delors Report (Delors 1996). This report has formed the basis for curricular reform around the AsiaPacific region (e.g. Bajunid 2008; Maclean 2002; Pene et al. 2002; Teasdale 2005), including the recently revised curriculum statement from the Vanuatu Ministry of Education (Vanuatu Ministry of Education 2010).

Finally, the inclusion of TEK in school may provide a means of addressing the underlying power imbalance that often exists between centralised, state-run systems of education and minority or indigenous groups. Education is a key 'regime of power' through which a cultures conception of truth is maintained (Van Eijck and Roth 2007), and as such can play a critical role in the marginalisation of epistemological diversity (De Sousa Santos 2007). In effect, this has meant that western-derived worldviews that promote values such as certainty, objectivity and instrumental rationality have dominated education systems and development rhetoric at the expense of local knowledge and practice (Teasdale 1995). In disregarding TEK and local content, formal education is argued to display 'systemic racism', and to foster separation between pupils and their community (Aikenhead 1993; International Council for Science 2002). The inclusion of TEK in formal school, therefore, may mitigate this power differential and promote local participation and empowerment in education (Aikenhead 2001).

However, proposals to include TEK in formal education are controversial. Commentators have argued that western systems of formal education are, in fact, 'antithetical' to systems of indigenous knowledge (Bates 2009; Thaman 2000), and have observed that the appropriation of TEK into school curricula may de-validate TEK by separating the knowledge from its cultural context (Sundar 2002). In other discussions, scholars have argued for the universal application of western scientific education (Matthews 1994), or have contended that educational reform on the basis of 'cultural difference' may obscure the fluid nature of culture and impede progress toward educational outcomes (Burnett 2007). 
In summary, academics and professionals from the fields of education, ethnobiology, and anthropology have called for the inclusion of TEK in formal education systems. However, such approaches may have negative consequences, which could lower the value of formal education or impact on the integrity of TEK. The integration of TEK and formal education will affect local TEK holders, parents, teachers, and education or cultural officials, all of whom are currently engaged in education and/or TEK conservation. The input of these stakeholders will be vital to the success of any such programme, but so far the literature does not include any examination of their perceptions.

The present study begins to fill this literature gap with a case study from Malekula Island, Vanuatu, by outlining the perceptions of ni-Vanuatu stakeholders on the value of TEK to formal education and vice versa. In this paper, I discuss the perceptions of interviewees related to three key questions: (i) Could TEK be legitimately included into the formal school system? (ii) How might this be achieved? (iii) What are the potential barriers to teaching TEK in schools?

\subsection{Methods}

\subsubsection{Setting}

This research was conducted on Malekula Island in the communities of Unmet, Tisvel, Dixon Reef, and Wintua. Please see Section 4.8 for further details. Please note that although the focus communities have different social and cultural characteristics, all interviews are treated as part of the same sample population. The cultural diversity of Malekula and Vanuatu means that my ability to generalise from case study data is limited; however, the issues faced by the focus communities and described herein are likely to be typical of issues facing rural populations throughout the country.

\subsubsection{Field methods}

Data were gathered using semi-structured interviews, which offer the flexibility to gather a wide range of information, and allow the interviewer to gain a more nuanced understanding than a structured questionnaire (Bernard 1998). I identified three key groups of stakeholders (Table 7.1): locally identified TEK experts and schoolteachers within the focus communities, and officials (including policy makers, VCC staff, and academics) in the capital Port Vila. These stakeholders were identified as being directly affected by any moves to integrate TEK and formal education or as being involved in forming education policy.

Interviewee selection was purposive and non-random, and contacts were gained through snowball sampling methods (Bernard 1998). For TEK experts and schoolteachers, my initial participants were selected through consultation with the VCC fieldworker in each community, and additional 
participants identified through recommendations of previous interviewees. In Port Vila, initial contacts were made through literature searches and key contacts at the VCC, and subsequent contacts through recommendations of previous interviewees. I recognise the bias inherent in snowball sampling, as the contacts one gains are dependent on the social networks of the gatekeeper informants (Bernard 1998).

Table 7.1: Interviewee characteristics for semi-structured interviews

\begin{tabular}{|c|c|c|c|c|}
\hline & & TEK experts & Teachers & Officials \\
\hline & Total $n$ & 27 & 12 & 10 \\
\hline \multirow{2}{*}{ Gender } & Male & 21 & 10 & 6 \\
\hline & Female & 6 & 2 & 4 \\
\hline \multirow{5}{*}{ Place } & Wintua & 4 & 4 & 0 \\
\hline & Dixon Reef & 10 & 2 & 0 \\
\hline & Tisvel & 8 & 3 & 0 \\
\hline & Unmet & 5 & 3 & 0 \\
\hline & Port Vila & 0 & 0 & 10 \\
\hline \multirow{7}{*}{ Position } & Chief & 11 & 0 & 0 \\
\hline & Fieldworker & 4 & 0 & 0 \\
\hline & Primary teacher & 0 & 7 & 0 \\
\hline & Secondary teacher & 0 & 5 & 0 \\
\hline & Academic & 0 & 0 & 5 \\
\hline & VCC staff & 0 & 0 & 3 \\
\hline & Education officials & 0 & 0 & 2 \\
\hline \multirow{3}{*}{ Age cohort } & $18-30$ years & 3 & 1 & 0 \\
\hline & $31-60$ years & 14 & 6 & 5 \\
\hline & $60+$ years & 10 & 5 & 5 \\
\hline
\end{tabular}

I lived in each of the four communities during 2008 and conducted interviews over this period.

Interviews were conducted as part of a larger research program and covered a number of topics (full schedule included in Appendix 11.6). The interview schedule was similar for each stakeholder group, but differed in the specific questions asked. For community-based interviews with TEK experts and teachers, the interview focussed around the magnitude and nature of changes in TEK and TEK transmission within the community; perceptions of the principal drivers of these changes; perceptions of the effects that these changes have had within the community; the ability of TEK to interact with the formal school system; and perceptions of specific areas of synergy. For interviews with Port Vila-based officials, questions focussed on the drivers of TEK and linguistic erosion; specific issues around curriculum and policy design; and barriers to the integration of local and formal education systems. Questions were piloted with VCC staff or fieldworkers to ensure salience. All interviews followed a list of key questions, but the approach was flexible to enable me to follow up leads and stories where pertinent. 
I conducted all interviews in Bislama or English according to the preferences of the interviewee. Interviews were conducted strictly according to a code of ethics adapted from the International Society of Ethnobiology (International Society of Ethnobiology 2006), and approved by the Human Ethics Committee at Victoria University of Wellington (approval number 16500) and the Vanuatu National Cultural Council under their Cultural Research Permit program. All participants were adult and gave their full, prior, informed verbal consent to the interview process. Participant identities remain confidential.

\subsubsection{Methodological limitations}

There were four key limitations to the methods outlined above. First, the use of in-depth qualitative methodology limits the sample size and thus constrains direct comparison with other studies or between groups within the data (for example, between the focus communities). Second, the sample selection was limited and excluded other relevant stakeholders (such as pupils and regional education officials) for ethical and logistical reasons. Third, the interview schedule did not identify specific mechanisms to include TEK in the school system.

Fourth, and critically, samples of TEK experts and teachers within the communities displayed significant gender biases. For the teachers grouping this may have been because there were more male teachers than female in the general population. For the TEK experts grouping, however, the bias resulted from methodological constraints, based on two primary factors. First, the initial interview targets (VCC fieldworkers) were all male, and tended to recommend men as experts for future interviews. Second, I found that women were less comfortable with the interview process and would often decline to be interviewed, possibly due to the presence of the researcher (a male New Zealander).

\subsubsection{Analysis}

Interviews were analysed using thematic coding based on an inductive approach. Coding was based on the approaches outlined by Miles and Huberman (1994) and Bernard (1998), and was completed in several distinct stages.

In the first iteration, I read all of the transcripts in order to gain a basic understanding of the responses. During this stage, I made a list of initial codes in the margins of the transcript, and used these labels to develop a general category scheme of participant responses. Second, I began to identify themes by sorting the initial scheme into concrete categories and subcategories. This categorisation reflected the frequency of response as well as the similarity between interviewees. Third, I re-read the transcripts to identify atypical and dissenting cases. The themes developed during 
this section form the paragraphs of the results section below. Last, I reviewed the themes and evaluated their relationship to the literature.

\subsection{Results}

Results are presented here according to the key questions set out at the end of the background section.

\subsubsection{Could TEK be legitimately included into the formal school system?}

All participants noted that TEK in Vanuatu had eroded over recent generations. This corroborates other data from the same research program, which (using a structured interview) indicated that 96\% of 120 participants around Malekula perceived TEK to be eroding (Section 4.9). Thirty of the 49 interviewees noted that the formal education system had played a key role in the erosion of this knowledge, alongside other key drivers such as church influence and community inattention. Participants noted that formal education drives TEK erosion through introducing new, competing, forms of knowledge; through promoting the use of English, French and Bislama over vernacular languages; and through a lack of integration with the wider community. As expressed by one elder at Unmet: "The kids go to school, and they catch some thinking that isn't really good - they learn knowledge, but they do not learn wisdom" (Male, 62, TEK expert).

Although $65 \%$ of respondents believed that TEK could be included in the formal school system, I found there were clear differences in the responses from different interviewee groupings (Figure 7.1). Teachers and community TEK experts were more likely than not to agree that TEK could be legitimately included in the formal education system. 


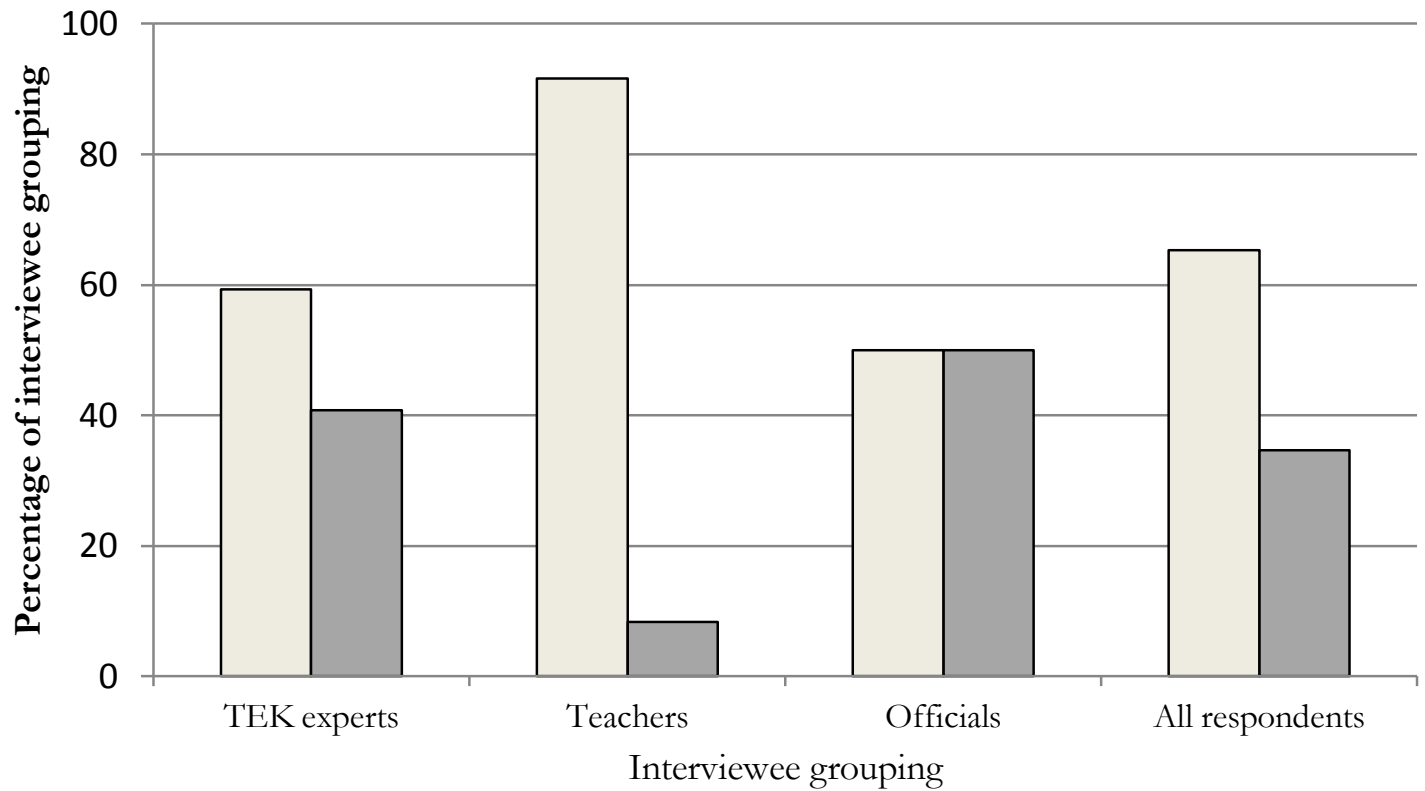

Figure 7.1: Percentage of respondents per grouping who support the teaching of TEK in formal school. Light bars indicate assent, dark bars indicate dissent

Participants who believed that TEK could be included in formal school systems gave a number of justifications, which coalesce into two main themes. The first group $(n=15)$ were not concerned about the negative consequences of such a move, and noted that TEK would be a valuable means of counterbalancing the western knowledge that is currently promoted by the school system. For example, one official described how TEK could enhance educational outcomes: "What I'm saying is there have been some things that have been learnt over centuries, over thousands and thousands of years, that have worked... and these are basically to do with interaction of people, and how they interwove [sic] all the different aspects of a community together to work together to achieve what we want today - and that's basically peace" (Male, c.60, Official). A teacher also stressed the role that TEK could play in education: "There are now two roads on offer [western and kastom], and it is hard for them to choose. People need their culture and traditions to be taught to them, to give them roots and make sure this choice is easier" (Female, c.40, Teacher).

The second group $(n=9)$ were pragmatic and were concerned with exploiting some of the characteristics of the formal education system to increase TEK transmission. The formal school aggregates pupils, and thus presents the opportunity of a captive audience for TEK transmission. As noted by an elder at Wintua: "...now that things are like this, it is too hard to call the kids to one place. They go this way and that all the time - but when that school bell rings, everyone goes there. That's why we need to teach customary knowledge at school' (Male, 65, TEK expert). Moreover, formal education represents a large time commitment, which participants perceived could profitably be used for TEK instruction. 
Figure 7.1 also indicates that a significant proportion of respondents (including $50 \%$ of officials) did not agree with the sentiments outlined above. A wide variety of reasons were given, which differed between the interviewee groupings; however in general, these participants were concerned that such a move would remove value, either from TEK itself or from the formal education experience.

TEK experts were concerned that integrating TEK and school might devalue the knowledge. Three key reasons were given: first, by inadvertently increasing the transmission of negative elements of kastom, including witchcraft and sorcery (cf. Rio 2010); second, by facilitating the teaching of gendered and secret aspects of TEK to a wider audience than would otherwise be intended; or third, by promoting the teaching of TEK by the wrong people, if appropriate teachers were unavailable. These participants noted that the transmission of TEK is inherently contextual, complex, and difficult to generalise into the school environment. This was wryly noted by one elder: “... [take] this leaf, for example, one of its medicines you can teach in public, but the other way of using it you definitely cannot. It is secret" (Male, 66, TEK expert). Moreover, there was also some concern that teaching TEK in a classroom, an essentially passive form of knowledge transmission, would disconnect the TEK from its practical reality. This viewpoint was supported by one official: "...the system as it is puts the kids in an artificial world that does not belong to them. But putting TEK into the school curriculum would force them to passively pick up the knowledge, rather than actively. This would change the knowledge... and surely that defeats the purpose of putting it in school?" (Female, c. 35, Official)

Other interviewees, in particular officials and teachers, focussed more on the impact that the introduction of TEK would have on the educational environment. There were two key concerns: the first, shared by two TEK experts as well as officials and teachers, was that teaching TEK at school would dilute the educational experience for pupils, in particular by interrupting the teaching of English or French. This would then impact on the ability of pupils to attain employment in urban centres such as Port Vila. One secondary school teacher noted "...it is part of the culture here that parents want their children to learn newer, better things that are different to what they know already. They want their children to be prepared for white-collar jobs...there are plenty who look down on the old ways as lower class" (Male, c.40, Teacher). Other teachers considered that such initiatives would compete for time in an already packed schedule. For example, social science, which is one area where TEK might be integrated, was only allocated 1 hour and 15 minutes of the school week, as opposed to 17 hours for Maths and English combined (McCarter, unpublished field notes).

Interviewees also mentioned several structural and epistemological barriers to the teaching of TEK in school, which are expanded on below. 


\subsubsection{How might TEK be integrated into the formal school system?}

When asked about appropriate subject material for the teaching of TEK in school, interviewees (mostly TEK experts) identified six discrete domains of knowledge that would be suitable for inclusion as subjects in the formal school system (Table 7.2). The first three (medicine, agriculture, and construction) refer to theoretical and practical TEK skills that participants felt are at risk of not being effectively transmitted and would translate well to the school environment. That is, they could conceivably be taught in a classroom through instruction from local resource people. Participants noted that the inclusion of TEK such as this in curriculum modules would increase intergenerational transmission of these subject domains; would make the younger generation more 'useful' in the community environment; and would increase their self reliance.

Table 7.2: TEK subject domains for inclusion in the school curriculum

\begin{tabular}{|c|c|c|c|c|}
\hline Subject domain & Featured skills & $\begin{array}{l}\text { Support } \\
\text { from TEK } \\
\text { experts } \\
(\mathrm{n}=27) \\
\end{array}$ & $\begin{array}{l}\text { Support from } \\
\text { teachers } \\
(n=12)\end{array}$ & $\begin{array}{l}\text { Support } \\
\text { from } \\
\text { officials } \\
(n=10) \\
\end{array}$ \\
\hline Traditional medicine & $\begin{array}{l}\text { Plant naming, illness naming and recognition, } \\
\text { medicinal preparation }\end{array}$ & 9 & 0 & 0 \\
\hline Agriculture & $\begin{array}{l}\text { Knowledge of traditional calendar, } \\
\text { agricultural techniques, respect for } \\
\text { appropriate social restrictions }\end{array}$ & 7 & 3 & 0 \\
\hline Construction & $\begin{array}{l}\text { Weaving bamboo walls, trying thatch with } \\
\text { vines, construction of 'kastom' style houses }\end{array}$ & 7 & 1 & 0 \\
\hline Resource management & $\begin{array}{l}\text { Recognition of appropriate ownership and } \\
\text { restrictions on natural resources }\end{array}$ & 5 & 2 & 2 \\
\hline Respect & $\begin{array}{l}\text { Fostering of appropriate attitudes and values } \\
\text { to property, people, and natural resources }\end{array}$ & 6 & 0 & 0 \\
\hline Vernacular language & $\begin{array}{l}\text { Linguistic skills, vernacular literacy, learning } \\
\text { of kastom stories and histories }\end{array}$ & 10 & 7 & 6 \\
\hline
\end{tabular}

In recommending the next two subject domains (resource management and respect) participants were more concerned with ensuring the transmission of appropriate norms and values to ensure cohesion within the community environment. This was considered critical for the effective governance of natural resources (such as through spatial and temporal restrictions on resource use known as tabu) and to foster appropriate attitudes to persons and property within the community. Participants commented that the individualistic ethic of the formal school system had resulted in a lack of respect for traditional institutions, and had increased incidences of theft or lack of appropriate 
deference to social hierarchies. There was some dissent on whether norms and values could be transmitted in school, which I return to in describing the barriers below.

Vernacular language was the most commonly discussed subject domain for potential inclusion in the school curriculum. The debate over the place of vernacular languages in the national curriculum in Vanuatu has continued for some years, and while some faltering steps have been made, there has been little real progress on the ground (Crowley 2000; Siegal 1996; Stahl 2004). Participants often noted that education in vernacular languages also implicitly teaches medicinal plant knowledge, social titles, agricultural techniques and the myriad other facets of TEK that are embodied in any particular language. Moreover, proficiency in a vernacular language fosters a sense of connection to place and land. This is true in both an esoteric and practical sense: if a person is not able to describe the boundaries of their family land in the vernacular, they risk losing much of their legitimate claim to that land. This is a worrying development in a time when customary title is often under dispute (Haccius 2011).

TEK experts noted that there would need to be changes to the current system for the appropriate inclusion of the subject domains from Table 7.2 in formal education. First, there would need to be systematic facilitation of access for local experts to the school system. School teachers are commonly non-local and are untrained in TEK and kastom, and there are many areas within the subject domains that would need to be taught by community-selected experts. Bringing resource men and women into the school system may also need to be supported by some form of cash payment. Second, the community experts would need to have some degree of autonomy over which aspects of TEK were taught at what time. This was mainly because, as noted above, there were concerns about negative aspects of TEK that would need to be tightly controlled from within the community. Third, there would need to be regular time created within the current curriculum setup to allow the effective transmission of TEK.

Several schoolteachers and officials $(n=7)$ added a qualification to their support for TEK in formal education by noting that the timing of such a move would be critical. In particular, they noted that vernacular language and/or TEK could be appropriately included in school at the primary level only. If included from an early age, participants noted that this would provide a 'base' upon which the other educational necessities could be built. Respondents felt that by the time pupils reached secondary school it was too late as the pupils were too old to acquire TEK and language effectively, and the time available in the curriculum is too limited. A senior teacher summed this position up: "...we should teach vernacular, but only at the lower levels. The kids come here, and the parents pay for them to be here, to learn English. We don't want to hold them back. At this stage, they need to gather the literacy and numeracy skills that will belp them in the future" (Male, c.50, Teacher). 
One official (Male, c.60) in Port Vila provided an instructive example of how TEK and formal school might be integrated from another island of Vanuatu (Futuna). For several years the interviewee has been working with the school board to break down the barriers between school and the community. They have been allowing regular slots within the school program for local experts to come and teach TEK, and have ensured community participation in the school board and regular contact with school authorities. The interviewee stressed that the focus has been on teaching values rather than specific skills. For example, he observed that being able to weave a mat is not the end goal of the teaching process, but rather the development of the patience and commitment to be able to complete the task. The interviewee noted that the program has been successful: as well as increasing intergenerational transmission of TEK, the school had also achieved one of the highest exam pass rates in Vanuatu. The philosophy of the approach is summed up in this excerpt: "... basically, we are going back to the simple things like [acknowledging] that the school is a small part of the community. It's not the other way around, the community is not a small part of the school. The school is a small part of the community, that means that the school fits in to the local environment, it fits into the local chiefs, it fits into how they do things."

\subsubsection{What are the possible barriers to teaching TEK in schools?}

Interviewees highlighted multiple barriers to the inclusion of TEK in formal education on Malekula, of which several have already been noted. These can be broadly divided into practical and epistemological barriers (Table 7.3).

Table 7.3: Barriers to the inclusion of TEK in school on Malekula

\begin{tabular}{|c|c|c|c|}
\hline Barriers to the inclusion of TEK in school & $\begin{array}{c}\text { Support from } \\
\text { TEK experts } \\
(\mathrm{n}=27)\end{array}$ & $\begin{array}{l}\text { Support from } \\
\text { teacher } \\
=12)\end{array}$ & $\begin{array}{l}\text { Support from } \\
\text { officials } \\
=10)\end{array}$ \\
\hline \multicolumn{4}{|l|}{ Practical barriers } \\
\hline \multicolumn{4}{|l|}{ Characteristics of formal school model } \\
\hline Inexpert teachers & 12 & 5 & 2 \\
\hline Lack of time in curriculum & 5 & 10 & 6 \\
\hline Lack of political support & 4 & 6 & 7 \\
\hline \multicolumn{4}{|l|}{ Characteristics of TEK } \\
\hline Increasing transmission of 'bad' kastom & 9 & 0 & 2 \\
\hline Payment and availability of resource people & 10 & 0 & 0 \\
\hline Lack of community support/ability & 11 & 4 & 1 \\
\hline Linguistic/cultural diversity & 3 & 7 & 4 \\
\hline \multicolumn{4}{|l|}{ Epistemological barriers } \\
\hline Mode of knowledge transmission & 9 & 5 & 6 \\
\hline Transmission of underlying values & 4 & 4 & 4 \\
\hline Different knowledge systems & 6 & 2 & 3 \\
\hline
\end{tabular}




\subsubsection{Practical barriers}

Practical barriers were associated both with characteristics of the formal school system and with the nature of TEK. Within the former grouping, issues with ensuring that the correct resource people are able to teach and that there was sufficient time in the curriculum have already been mentioned. An additional key dynamic is the lack of consistent political support for TEK and for vernacular language education. This was perceived to be a problem by the majority of officials and teachers. Vanuatu's political landscape is highly fractured, and governments and ministers change regularly. There are splits between Anglophone and Francophone sections of government, and if policy changes are enacted they often lack the resources to be implemented fully. Moreover, interviewees believed that until recently donor priorities (especially those of New Zealand and Australian aid agencies) had steered educational policy away from contextualised, indigenised, ni-Vanuatu education.

The sheer cultural diversity of Malekula was also cited as a barrier to the effective integration of TEK into the formal school system. This is particularly true in the secondary school setting, where students commonly come from around Vanuatu, and where multiple vernacular language groups are represented in a single classroom. Even in primary education (where only one language group is usually represented), there may be many clan groups present, which is an artefact of the resettlement patterns that followed mission establishment around the island (Section 4.7). Three of the TEK experts expressed concern that political allegiances would mean some families would be marginalised, or one dominant language would be privileged over others. As one of these TEK experts at Dixon Reef, where one vernacular language (Novol) is dominant, noted: “...there are many different languages here, and if they do not stand strong, then Novol will come on top of each of them" (Male, c.60, TEK Expert).

\subsubsection{Epistemological barriers}

Interviewees also cited a number of epistemological barriers to the inclusion of TEK in the school system. Some of the most commonly expressed reservations were associated with differing methods of knowledge transmission between the formal school system and a traditional system. Formal school is based around a teacher-centred model in which one or two instructors dispense public knowledge to many learners, regardless of clan affiliation, gender, or age. This model was considered to be at odds with systems of TEK by several interviewees, who noted that much TEK was private and was held by particular family groups or individuals. Moreover, traditional routes of knowledge transmission would have been based around vertical (parent-child) means rather than horizontal (within peer groups) or oblique (one instructor to many learners) means. By way of example, in traditional medicine some use domains (such as that of how to treat common ailments such as 
headaches) are considered common property and could be taught at school. However, treatment of more serious illnesses or those with traditional aetiologies are the domain of certain individuals who have earned the right to use them from the previous holder of that knowledge.

More broadly, then, there was a common perception that while the Western-derived formal education system is based in an ethic of every student having the right to know everything, this is not always congruent with customary systems of knowledge transmission and acquisition. Moreover, practice and ownership of TEK was considered to be bound by an ethic of respect and by social norms that existed outside the transmission of the knowledge itself. The following quotation demonstrates that several interviewees considered the school system to be a limited conduit for the transmission of such values: "I mean, if we are going to think we can teach these values by someone who is in the community coming to talk to them, basically what they can only teach are the things that you can see outside. But the real thing that should drive everything else... you cannot teach it like that. You teach by way of life... and if we don't do that, then it won't work..." (Male, c.60, Official)

Underlying such concerns is a common conception that the two systems of knowledge transmission are fundamentally different. Ideally TEK is embedded in everyday life, whereas formal school is perceived as a discrete entity that exists outside the framework of the village. Whilst the transmission of TEK from teacher to learner is based in the practical reality of the lived environment, knowledge in school is theoretical, conceived and stored in paper and books. As such, those interviewees who did not support the teaching of TEK in school often noted that it was impossible to teach TEK adequately in the academic, formal context, as this would remove the connection between knowledge the teaching of practical skills. This fundamental disconnect was expressed by a female TEK expert: “... in the time before, we didn't need to go to school to learn traditional knowledge - it was just life..." (Female, 30, TEK Expert).

\subsection{Discussion}

Interviewees outlined a number of key areas of synergy between the formal school system and TEK. Moreover, they noted that the flexibility to incorporate distinct domains of TEK could add value to the school curriculum and contribute to the maintenance of kastom. As such, the data corroborate the key arguments raised in the background section regarding the potential of TEK to contribute to formal education systems. However, the epistemological and practical barriers to teaching TEK at school suggest that there is reason for caution. In particular, interviewees noted the potential for adverse effects on both traditional knowledge and the outcomes of formal education. 
The majority of interviewees noted that the formal school system does not fully meet contemporary needs or sustain traditional culture and TEK. This supports assertions made by some ni-Vanuatu writers, who contend that the education sector should be contextualised through the inclusion of local and indigenous content (e.g. Baeraleo 2004; Hickey 2005; Niroa 2004; Regenvanu 2002; Regenvanu 2005). Whilst the inclusion and support of traditional culture and TEK is only one aspect of the education system that needs updating, Vanuatu's formal school system has been described as an 'alienation agent' that has a tendency to remove children from the context of their traditional culture at an early age (Niroa 2004). Moreover, comments during the interview process indicate support for the idea that Vanuatu schools may perpetuate a 'psychology of dependency' by devaluing local knowledge relative to western knowledge and modes of learning (Regenvanu 2005).

These findings also support the assertion that, prima facie, TEK could add value to the school curriculum in Vanuatu. This is no particular surprise, given that TEK has been shown to have immense value across a number of spheres over the past decades (Berkes 2008). However, the value of TEK in this regard is critical, as countries across the Pacific region (including Vanuatu) are currently debating and enacting curricular reform (Bajunid 2008; Maclean 2002; Teasdale 2005).

Crucially, I note there are specific synergies between the various ways in which interviewees suggested that TEK might complement the school curriculum and the model of UNESCOmandated reform proposed in the Delors Report (1996). This report suggested education policy should be reorganised around four 'pillars': learning to know, learning to do, learning to live together, and learning to be. The western model of education tends to focus on the first two pillars. However, the Delors Report accorded equal importance to latter two and emphasised the need for students to be aware of their values and place in the world. This report has had a significant influence on the direction of curricular reform in the region (Teasdale 2005), and if TEK adds value to curricula by contributing to the four pillars it would strengthen the case for its inclusion in formal education systems.

The first two pillars ('learning to know' and 'learning to do') are concerned with cultivating a desire and ability to learn, and the skills to reflect learned knowledge with innate competencies. These two pillars have been the focus of education, both formal and informal, over the past decades in Vanuatu and the Pacific more broadly (Teasdale et al. 2005). However in the Pacific context scholars have heavily criticised the status quo, noting that the knowledge and skills taught in formal school systems around the region are not representative of the depth and diversity of local knowledge (Teasdale and Ma Rhea 2000; Thaman 2002). Moreover, these scholars contend that conventional school curricula in the Pacific region are rooted in a foreign worldview that fails to build on existing competencies and impedes the transmission of traditional knowledge (Sanga et al. 2004). 
Interviewees noted specific domains of knowledge and skills (such as traditional medicine) that form discrete areas in which TEK could contribute to the school system. The identification of these focal areas suggests that the introduction of TEK into education might be a key step in localising the content and process of curricular delivery, thus strengthening the first two pillars as suggested in the Delors Report. Any such move would be supported by recent work which has documented distinct benefits of contextualising formal education using local knowledge (Ruiz-Mallen et al. 2010; Taylor and Mulhall 2001). Moreover, the maintenance and revitalisation of TEK knowledge and skills may have a direct impact on the adaptive capacity of the communities in question: for example, traditional methods of house construction have been shown to be a critical element of resilience to extreme weather events in other areas of Vanuatu (Campbell 2006).

However, TEK may be able to add value most significantly to the latter two pillars ('learning to live together' and 'learning to be'). The strengthening of these two pillars provides a particular challenge to curricular reform, as it necessitates a shift away from teacher-centred, exam-based learning, and may also require community support and participation (Teasdale et al. 2005). The inclusion of these pillars in the Delors Report is an acknowledgement that education should consist of more than the passing of decontextualised knowledge and skills, and should contribute to the formation of identity in the individual and the eventual development of wisdom. These two pillars cannot be attached as discrete elements in the curriculum, and must be woven throughout each subject area (Teasdale et al. 2005).

I contend that the inclusion of TEK (especially the participant-identified domains of 'respect', traditional resource management institutions, and vernacular language) has potential to strengthen significantly the ability of education to address the latter two pillars. As several interviewees noted, the institutions that surround TEK are the key means of maintaining order and governing natural resources in these communities. The recognition and incorporation of these institutions, including the appropriate respect and acknowledgement of leaders, into the school curriculum might be a key way of ensuring that 'learning to live together' is adapted to the village environment. In turn, this would assist in the promotion of resource management for resilience, and may foster the understanding of ecological processes (Berkes et al. 2000). Moreover, the integration of vernacular language and TEK teaching in the formal school system would ensure that students developed a sense of membership of their particular clan, village and island (Niroa 2004). 


\subsubsection{Barriers to including TEK in the formal education system}

Although there is potential for TEK to add value to the existing school curriculum in Vanuatu, its introduction may be constrained by multiple challenges. Interviewees noted three key tensions inherent in the geographic and social context of TEK:

1. Diversity vs. centralisation

TEK, as a localised entity, is unlikely to be widely applicable outside the environmental and social context in which it has evolved (Agrawal 1995). Therefore, school curricula that involve TEK must be flexible enough to incorporate local views and empower TEK holders, despite emanating from a central government. This may involve the use of specific place-based assessments (cf. Zent 1999), but this process would have to be thorough and carefully managed in a culturally diverse nation such as Vanuatu.

2. Public vs. private knowledge

Intellectual property becomes a key concern with any attempt to formally include TEK in 'public' education. TEK is often intimately connected with social order and family groupings, and may not be appropriately shared with the wider community. Therefore, community heterogeneity must be taken into account (Agrawal and Gibson 1999). This also indicates that the inclusion of TEK in formal education is likely to be only one of a range of tools needed for the maintenance and revitalisation of traditional knowledge.

3. Vertical vs. horizontal knowledge transmission

In other areas of the world, cultural transmission of TEK has been shown to be predominantly vertical (parent to child) (e.g. Ohmagari and Berkes 1997), and this is likely to be the case on Malekula. Integrating TEK into formal school may shift the mode of knowledge transmission from vertical to horizontal (within peer groups) or oblique (one instructor from the parental generation to many younger learners). This may result in a fundamental change in the structure and content of TEK, because the type of transmission pathway can influence the characteristics of that body of knowledge. For example, while vertical transmission results in slow rates of adaptation, horizontal and oblique methods can result in rapid diffusion and spread of new ideas (Hewlett and CavalliSforza 1986). A crucial facet of this tension is the shift from oral to written forms of knowledge transmission.

Perhaps more importantly, however, these findings draw attention to significant epistemological barriers to the integration of TEK and the formal school curriculum. Although the boundaries between indigenous knowledge and western knowledge have been argued to be largely arbitrary and 
unhelpful (Agrawal 1995), it is clear that interviewees considered there are fundamental differences in the two systems of knowledge transmission on Malekula. This is corroborated by research indicating that TEK transmission is usually experience-based, learner-centred, and acquired through social interactions such as play, in contrast to knowledge transmission in the formal school system (Hunn 2002; Lancy 1996).

Within the Pacific, other research has found that imported education systems are, indeed, 'antithetical' to local means of indigenous education (Thaman 2002). Such work has argued that irrespective of the content of the curriculum, the makeup and structure of the school mean that it transmits essentially foreign values (Teasdale et al. 2005; Thaman 2002). These values are transmitted through the ethic of the learning environment, as Sundar (2002) notes in the global context: “...critical education theorists have long since laid to rest the idea that curricula involve an innocent transmission of 'knowledge' that is not at the same time inflected by race, class, or gendered assumptions, or that pedagogy does not involve moral projects of transformation" ( $\mathrm{p}$ 374). As such, it would be extremely challenging to teach TEK in a western setting in a way that would not emphasise those foreign values, which may in the process invalidate the TEK (Heckler 2007). This was reflected in the comments from interviewees who were concerned that the inclusion of TEK in the school curriculum might implicitly erode traditional means of transmission.

Underlying all the barriers are issues concerning the power and sovereignty of local and indigenous peoples over the education of their younger generations. The validation and incorporation of knowledge in the formal school system is an immensely powerful act, as this knowledge has a claim to 'truth' that others do not (Van Eijck and Roth 2007). The holders of TEK should be in control of this process, as actors in positions of power (such as academics or policy-makers) can influence the choices that local people make about what is desirable and valuable in their own paradigms (Heckler 2007). This will, in turn, require significant attention to the ways in which curricula are developed and implemented, as " ... without explicit and continuing attention to how power structures knowledge, it will remain impossible to achieve the aim of working in the interests of indigenous or other marginal peoples" (Agrawal 2002: p 295). As such, the conservation of TEK and the strengthening of education curricula should avoid a focus on specific pieces of knowledge in isolation from their cultural context. A more appropriate focus may be on sustaining the institutions and worldviews in which that knowledge is embedded (Agrawal 1995).

\subsubsection{Moving forward with TEK maintenance and revitalisation}

There are, then, significant issues that might impact on the value that formal education systems might have to TEK maintenance and revitalisation. I argue, however, that approaches that facilitate a 
high level of local participation in teaching and unit design may offer profitable future pathways for TEK maintenance.

One such approach, implemented in one school on the island of Futuna, was noted in the results (Section 7.3.2). Another example is the VCC's recent design of a series of units for years one to eight, which aim to involve community members and incorporate TEK into the science curriculum (Baeraleo 2010; Regenvanu 2004). These units do not seek to document specific details of TEK within the curriculum, but rather focus on encouraging pupils to seek out experts in their communities and discuss various aspects of the natural world. They also contain a dedicated teachertraining component to ensure that teachers have the skills to facilitate increased contact with the community. At the time of writing, the units were being distributed to regional education officials around the country and were intended for use in the 2011 year. No information is available at the present time as to their success or otherwise, however these units represent a promising and innovative means of increasing intergenerational TEK transmission.

Because formal school is unlikely to be able to maintain all aspects of TEK, other means may also be necessary. Of the other available means for TEK maintenance, in situ revitalisation efforts appear to hold the most promise (Pilgrim et al. 2009). Globally, in situ TEK maintenance initiatives have been created with a wide range of objectives, including the promotion of vernacular language, campaigns for human and land rights, and increasing the consumption of traditional foods (Pilgrim et al. 2009). Other approaches seek to meld biological and cultural conservation goals for integrated biocultural conservation (see Maffi and Woodley 2010). On Malekula, a series of local 'kastom schools' (small local organisations, independent of the formal school, for the teaching of kastom and TEK) provide an interesting case study of in situ TEK conservation (Chapter Eight). In general, approaches that address the fundamental issues of power imbalance, control over intellectual property, and TEK erosion have promise. However, as noted, there is a real need for more research attention to the challenges inherent in maintaining and revitalising TEK, vernacular language, and cultural continuity more broadly.

\subsection{Conclusions}

I find that TEK may be able to add value to the formal school system in Vanuatu, especially with regards to curriculum reform via the model set out in the Delors Report. In addition, this may assist in the maintenance of ethnobiological knowledge. However, I note that the value of formal education to TEK is less assured, and that overcoming the practical and epistemological barriers outlined above will require considerable effort. Indeed, to do so may require a substantial redesign of the entire school system, to allow for not just the dissemination of other forms of knowledge but 
also to empower other ways of being, knowing, and learning. However, critically, I note that the desire for such a radical change in education policy may not always exist at the local level. Ideally then, local people would have more power to determine the content and structure of the education system.

Education reformists, ethnobiologists, and practitioners of cultural conservation have all called for the inclusion of TEK in the formal school system. However, little research has occurred examining the feasibility of this approach. What is now needed is more detailed research on how to cope with the kinds of barriers identified here, or to determine if other modes of TEK conservation would be more practical. As a discipline, ethnobiology is in a unique position to assist the conservation of biocultural diversity, and a more systematic examination of the potential options for the maintenance and revitalisation of TEK will be a vital contribution over the coming years. 


\section{The in situ}

maintenance of TEK on

\section{Malekula}

\subsection{Introduction}

The preceding chapters have explored aspects of the variation, transmission, and maintenance of TEK on Malekula Island. In Chapters Five and Six, the data indicate that aspects of TEK are at risk on Malekula, and that modern environmental and social changes have impacted on intergenerational systems of TEK transmission. The maintenance of TEK therefore becomes a critical issue, and Chapter Seven began discussing this by investigating the value of TEK to formal education curricula. This chapter continues the theme by examining another potential means for the maintenance of TEK, the in situ approach. Here, I examine case studies of in situ TEK maintenance from Malekula Island in the form of three 'kastom schools', and in doing so address objective 3(b): 'to assess community perceptions of in situ TEK conservation by examining the triggers and barriers to three local kastom schools'. This chapter fills a key literature gap, as in situ approaches for TEK maintenance have yet to be discussed in detail in the ethnobiological literature, despite being proposed by a variety of commentators (Agrawal 1995; Zent 1999).

I begin this chapter by outlining the relevant literature, focussing on the theoretical importance of approaches for the in situ maintenance of TEK. I then briefly introduce the kastom schools, and note their key characteristics and current status. Next, I outline the qualitative methodology and analytical techniques used here, before presenting results in two parts that centre on the triggers and the barriers to the schools. I close this chapter with a detailed discussion section that focuses on the implications of some of the epistemological barriers to in situ TEK maintenance on Malekula, which effectively problematise simplistic notions of TEK conservation. 


\subsubsection{International in situ TEK maintenance}

TEK is threatened worldwide as systems of cultural and biological diversity decline toward 'monocultures of the land, people, and mind' (Pretty 2011: p128). Recent quantitative work has shown that TEK can erode in association with various indicators of globalisation, including formal schooling (Cruz García 2006), integration into the cash economy (Godoy et al. 1998), and linguistic shifts (Benz et al. 2000; Nettle and Romaine 2000). Published research within the Pacific has given evidence TEK erosion (e.g. Brosi et al. 2007; Case et al. 2005; Lee et al. 2001), and some of these findings were corroborated for Vanuatu by the evidence presented in Chapter Five. The erosion of TEK may result in a net increase in the vulnerability of social-ecological systems through impacting on social and human capital (Pretty 2011). In response, Zent (1999: p 91) has proposed that researchers should heed an 'ethnoecological imperative', which refers to the urgent need to document and conserve traditional knowledge and management systems before they disappear. This sentiment has been echoed in a number of other works (Brosi et al. 2007; Harmon 2002; Harrison 2007; Heckler 2002; Maffi 2004; Zarger 2002), and is reflected in the code of ethics of the International Society for Ethnobiology, which compels researchers to “... [take] active measures to protect and to enhance the relationships of indigenous peoples, traditional societies and local communities with their environment and thereby promote the maintenance of cultural and biological diversity" (International Society of Ethnobiology 2006: principle 10).

Several approaches for TEK maintenance have been suggested in the literature. These have included large scale top-down approaches, such as the integration of TEK and formal school systems (Heckler 2002; Kimmerer 2002; Zent 1999); or the creation of ex situ online databases for the storage and dissemination of knowledge (e.g. TKDL 2011; UNESCO 2011; World Intellectual Property Organisation 2011). However, both approaches have also been criticised. Top-down approaches may negate local diversity, may exacerbate existing power differentials, and can be slow to change and adapt (Bates 2009; Zent 1999), while ex situ approaches have been criticised for overlooking the contextual and place-based nature of TEK, and for their potential to facilitate the private appropriation of knowledge from the source communities (Agrawal 1995; Agrawal 2002; Zent 1999). The findings presented in Chapter Seven support some of the arguments against top-down TEK maintenance in noting they may only be appropriate for certain, empirical, aspects of TEK.

Consequently, in situ approaches may represent some of the most promising options for the maintenance and revitalisation of TEK. Broadly defined, these are approaches to TEK maintenance that seek to maintain and revitalise knowledge and practice within the source community (Agrawal 1995). These approaches have taken a number of forms, from biocultural conservation schemes which seek to protect local cultural heritage in concert with biodiversity management (Maffi and 
Woodley 2010) to community-based revitalisation efforts that have been rooted in diverse subject domains, such as linguistic revival or the support of traditional food cultures (Pilgrim et al. 2009; Pretty 2011; Singh et al. 2010). Ideally, these approaches are initiated by the source community, and involve predominantly local actors.

In situ and local approaches for TEK maintenance may have a number of benefits over top-down interventions. For one, they work through existing social networks, and so may be effective at improving the intergenerational transmission of knowledge (Pilgrim et al. 2009). Moreover, they may be able to strengthen traditional pathways for cultural transmission, and ensure the use of locally appropriate settings and strategies for knowledge transmission (Bates 2009). This is an important concern given some of the issues raised in Chapters Six and Seven: the former noted that both the setting and the strategy for knowledge transmission were critical, while the latter found that inappropriate techniques for the transmission of knowledge have potential to alter the nature of the TEK itself (McCarter and Gavin 2011). In situ approaches may also be well positioned to assist in the retention of intellectual property rights by the holders of the knowledge themselves, and may be cost effective, relative to the high cost associated especially with top-down governmental interventions (Agrawal 1995). Overall, the key benefit of in situ approaches is that they are likely ensure that power remains with the holders of the TEK, thus ensuring that empirical knowledge is not separated from the worldview, place, and culture within which it is encapsulated, and so playing a key role in the facilitation of cultural self-determination (Pilgrim et al. 2009). Given that TEK is adaptive and dynamic, and that the drivers of TEK change are likely to be locally specific, the consideration of the wider political, institutional, and cultural dimensions of traditional knowledge (the 'cultural landscape' of TEK) will be critical aspects of any future TEK maintenance (Berkes 2008).

Recent documentation efforts have been valuable, and have focussed on the description of in situ schemes for biocultural conservation (Maffi and Woodley 2010) or cultural revitalisation (Pilgrim et al. 2009). We now know significantly more about the ways in which such programs may be designed, and something of the specific benefits that can accompany in situ TEK maintenance. However, there are few published examples of programs that focus explicitly on developing mechanisms and processes for the revitalisation of TEK (such as Plotkin 1993). This is a critical gap, as the establishment of these programs in other areas will hinge on creation of the necessary conditions for in situ TEK maintenance and mitigation of key barriers. Moreover, the evidence presented in the previous chapter indicates that there is potential for TEK conservation efforts to have negative consequences, meaning that research into the epistemological basis for TEK maintenance efforts will be a valuable contribution. Therefore, there is a need for documentation and discussion around the effective design of measures for TEK maintenance, as well as the factors which may promote or 
hinder the establishment of such schemes. This chapter begins to fill this literature gap by examining three case studies of in situ TEK maintenance from Malekula.

\subsubsection{In situ TEK maintenance in Vanuatu: The kastom schools}

Between 2008 and 2009, community members at Lawa, Dixon Reef, and Tisvel initiated 'kastom schools' in their communities. These schools are, in essence, small, locally-based organisations that aim to facilitate the transmission of traditional knowledge and culture within their community (Table 8.1). All have a strong focus on TEK, and aim to teach a range of skills ranging from natural resource management to the preparation of medicinal plants. However, all of the kastom schools have struggled to operate consistently.

Table 8.1: Key characteristics of three kastom schools on Malekula Island

\begin{tabular}{|c|c|c|c|}
\hline & Lawa & Dixon Reef & Unmet \\
\hline Pupil age & $\begin{array}{l}\text { All ages, in particular school } \\
\text { leavers returning to the } \\
\text { community }\end{array}$ & $\begin{array}{l}\text { Primary school age pupils, } \\
\text { with plans to expand }\end{array}$ & $\begin{array}{l}\text { All school age pupils (5-15); } \\
\text { plans to include pre-school in } \\
\text { future }\end{array}$ \\
\hline Location & $\begin{array}{l}\text { Separate from the village, } \\
\text { located on a hill c. } 15 \text { min walk }\end{array}$ & Within village & $\begin{array}{l}\text { Within village; however some } \\
\text { activities to be taught in } \\
\text { private }\end{array}$ \\
\hline $\begin{array}{l}\text { Links with } \\
\text { formal } \\
\text { school }\end{array}$ & Separate from formal school & $\begin{array}{l}\text { TBC: ideally a separate, } \\
\text { delegated, building, but could } \\
\text { run through formal school }\end{array}$ & $\begin{array}{l}\text { Within the formal school: } 1 \mathrm{hr} \\
\text { per week per age group }\end{array}$ \\
\hline Key subjects & $\begin{array}{l}\text { Ethnomedical knowledge and } \\
\text { practice; vernacular language; } \\
\text { appropriate terms for familial } \\
\text { respect }\end{array}$ & $\begin{array}{l}\text { Initial focus on language, then } \\
\text { different aspects of culture and } \\
\text { traditional medicinal use. }\end{array}$ & $\begin{array}{l}\text { Kastom dancing and dress, } \\
\text { handicrafts, some medicinal } \\
\text { use. }\end{array}$ \\
\hline Governance & $\begin{array}{l}\text { VCC fieldworker with advisory } \\
\text { group }\end{array}$ & $\begin{array}{l}\text { Elected committee (three men, } \\
\text { two women) }\end{array}$ & $\begin{array}{l}\text { Two elected committees: one } \\
\text { male, one female }\end{array}$ \\
\hline $\begin{array}{l}\text { Teaching } \\
\text { staff }\end{array}$ & $\begin{array}{l}\text { VCC fieldworker and invited } \\
\text { resource people (male and } \\
\text { female) }\end{array}$ & $\begin{array}{l}\text { Two teachers, one male and } \\
\text { one female }\end{array}$ & $\begin{array}{l}\text { One main male teacher, with } \\
\text { specialist male and female } \\
\text { teachers bought in for } \\
\text { gendered skills }\end{array}$ \\
\hline $\begin{array}{l}\text { Other } \\
\text { features }\end{array}$ & $\begin{array}{l}\text { A full medicinal plant garden } \\
\text { and traditional house to teach } \\
\text { building techniques }\end{array}$ & $\begin{array}{l}\text { Planning to continue focus on } \\
\text { sand-drawing and traditional } \\
\text { forms of communication }\end{array}$ & $\begin{array}{l}\text { Aiming to start a 'kastom } \\
\text { museum' to support the kastom } \\
\text { school through tourist dollars }\end{array}$ \\
\hline Funding & $\begin{array}{l}\text { Small grant from French } \\
\text { Embassy; future funding } \\
\text { through sales of kava and } \\
\text { cacao }\end{array}$ & $\begin{array}{l}\text { To be funded through small } \\
\text { grant from VUW, community } \\
\text { fundraising, kastom economy } \\
\text { items }\end{array}$ & $\begin{array}{l}\text { To be funded through a small } \\
\text { grant from VUW, community } \\
\text { fundraising, eventually by } \\
\text { proceeds from kastom museum }\end{array}$ \\
\hline
\end{tabular}

There are a number of key differences between the three kastom schools. First, the management structure of each school is different. In Lawa the VCC fieldworker (responsible for the establishment of the school) is in charge, with a small advisory group, whereas at Dixon Reef and Unmet elected committees decide on curriculum subjects, appoint resource people to teach, and are in charge of administration. Second, topics for consideration are subtly different between the schools: at Lawa and Dixon Reef, the emphasis is on language and TEK, whereas at Unmet the focus is on ceremonial 
preparation, dance, and dress. Third, and critically, the schools all differ with regards to their position regarding the formal school within the village: at Lawa the kastom school is completely separate; at Dixon Reef they may utilise the infrastructure of the formal school (classroom, blackboard), however the kastom school committee are hoping to construct a separate location; and at Unmet the kastom school will be taught from within the formal school, with the approval of the headmaster. Finally, the school at Unmet is unique for having a vision of a 'kastom museum'. The vision of the organisers is for this museum to attract tourists to view kastom dances and performances, the proceeds of which would be used to help fund the kastom school. This is an aspirational goal, however fundraising is proceeding for construction.

Two of the three schools (Dixon Reef and Unmet) arose from two workshops co-hosted by the VCC and Victoria University of Wellington (VUW) in 2008. These workshops were held to discuss the variation and conservation of TEK around Malekula, and included 35 participants from 17 language groups around Malekula. ${ }^{26}$ One session of each workshop was left open for the discussion of TEK conservation amongst the participants, and it was during these sessions that the idea of kastom schools was initially mooted. On returning to their communities, both participants (also VCC fieldworkers

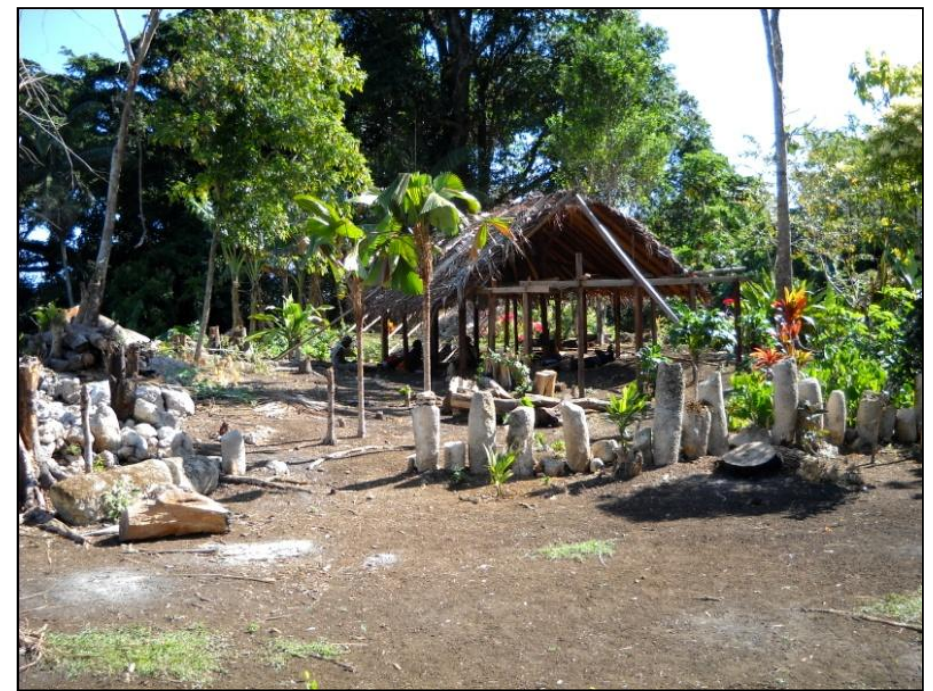

Plate 8.2: The kastom school at Lawa and community chiefs) formed kastom school committees and began to establish the schools. The third kastom school (at Lawa) was established primarily through the instigation of the VCC fieldworker in that community, who did not attend the VCC/VUW workshops in 2008. The mode of formation of the schools is a critical locus for discussion that I return to below.

This chapter does not seek to describe the kastom schools beyond the general outline above, because the models used here are not likely to be exactly applicable elsewhere. Indeed, each of the kastom schools is a unique product of the socio-ecological conditions of the community in which it is situated, and responses for TEK maintenance in other communities worldwide should be similarly

\footnotetext{
26 These workshops were part of a separate research program, and were co-hosted by Victoria University of Wellington and the VCC under the direction of Dr Michael Gavin. Their aim was to examine island-wide patterns of TEK variation, utilising the fieldworker network from around Malekula. As such, discussions of TEK maintenance were not an original research objective, but rather something that developed organically as the workshop series progressed.
} 
locally adapted. Rather, this paper seeks to contribute by identifying the triggers and barriers to their formation and function, and so provide applicable lessons to other such projects in other locations. How, then, did the kastom schools arise? What are some of the key challenges that they have faced, and are these surmountable? In sum, do they represent a feasible means of maintaining TEK? I begin to answer these questions below.

\subsection{Methods}

\subsubsection{Setting}

This research was based in Lawa, Dixon Reef, and Unmet. Please see Section 4.8 for more information on study sites and site selection.

\subsubsection{Field methods}

I utilised semi-structured interviews to build a picture of the triggers and barriers to the continued functioning of the kastom schools. Interviews typically lasted between 45 and 90 minutes, and were conducted in Bislama (schedule included in Appendix 11.8). Interviews covered a range of subjects including the nature of recent changes in ethnobiological knowledge; drivers of these trends; as well as exploring the nature, functioning, and challenges associated with the kastom schools.

I adapted a code of ethics from the International Society for Ethnobiology (International Society of Ethnobiology 2008), and my research received approval from the Victoria University Human Ethics Committee (approval number 16500) as well as from the Vanuatu Cultural Centre, under their Cultural Research Policy (Vanuatu Cultural Centre 2011). All participants were adult (over 18), were fully informed, remain confidential, and gave the verbal consent to the interview process. Please see Section 3.3 for more information and justification of my ethical approach.

\subsubsection{Participants}

I conducted fifteen detailed semi-structured interviews with key informants about the kastom schools, as well as four group interviews with the kastom school committees (two of these were in Unmet, where there is a separate woman's committee). Participant selection for this section of the research was purposive and non-random, and focussed on identifying the key actors within each of the three kastom schools. Participants were identified by gatekeeper informants (in each case VCC

fieldworkers), who were themselves interview subjects. I stress that my intention was not to conduct a thorough stakeholder analysis of the kastom schools, but rather to discuss with the key proponents of the schools the triggers and challenges they had faced. 
The mean age of participants was 51 and ranged between 29 and around 70 (older participants are often not sure of their exact age). Participants were evenly distributed between communities with five formal interviews in each study community. Data collected in interviews were supported by extensive informal discussions with community members and leaders in each community, as well as in several neighbouring communities.

My sample selection risks several inherent biases. Any snowball sampling is liable to result in one interviewing only those who fall within the social networks of the original informant (Bernard 1998). In this case, because I only spoke to key protagonists, I am limited in my ability to generalise the findings across the entire community or to detect dissent. The sample selection is, however, sufficient to attain my main goal of describing the triggers and barriers to the schools, according to the protagonists.

The sample also displayed a significant gender bias: of the individual interviewees only one was female. As noted other chapters, this was partially due to some women being uncomfortable with being interviewed. More significant, however, was the gender bias that existed within the kastom school committees and power structures, which meant my sampling strategy (in focussing on the key actors within the schools) intrinsically targeted male participants. I return to this point in the discussion; at this stage suffice to say that this may represent a weakness of the kastom school model, which is rooted in local power dynamics and thus is dependent on the preferences of the key establishing actors.

\subsubsection{Analysis}

The analysis of the interview data served to address my key focus of describing the triggers and barriers to the kastom schools. Following Miles and Huberman (1994) and Bernard (1998) I identified and categorised all participant responses regarding the kastom schools.

Interview coding was completed in several distinct iterations. First, I read through the entire transcripts in order to gain a basic understanding of the responses. During this stage I made a list of initial codes in the margins, and used these labels to develop a general category scheme of participant responses.

Second, I began to identify themes by sorting the initial scheme into concrete categories and subcategories. This categorisation reflected the frequency of response as well as the similarity between interviewees, and at least half the respondents had to note a theme for it to be included in the final categorisation. Next, I re-read the transcripts looking for atypical or unexpected cases. The themes developed during this section form the subheadings of the results section below. 
Third, I reviewed the themes and evaluated their links to the literature, both regarding the conservation of ethnobiological knowledge and the nature of kastom and culture in Vanuatu. In doing so I was able to generate several key themes that illuminate something of the participants' responses to the kastom schools and their ongoing importance.

\subsection{Results}

Triggers and barriers to the kastom schools are summarised in Table 8.2.

Table 8.2: Summary of triggers and barriers to kastom schools

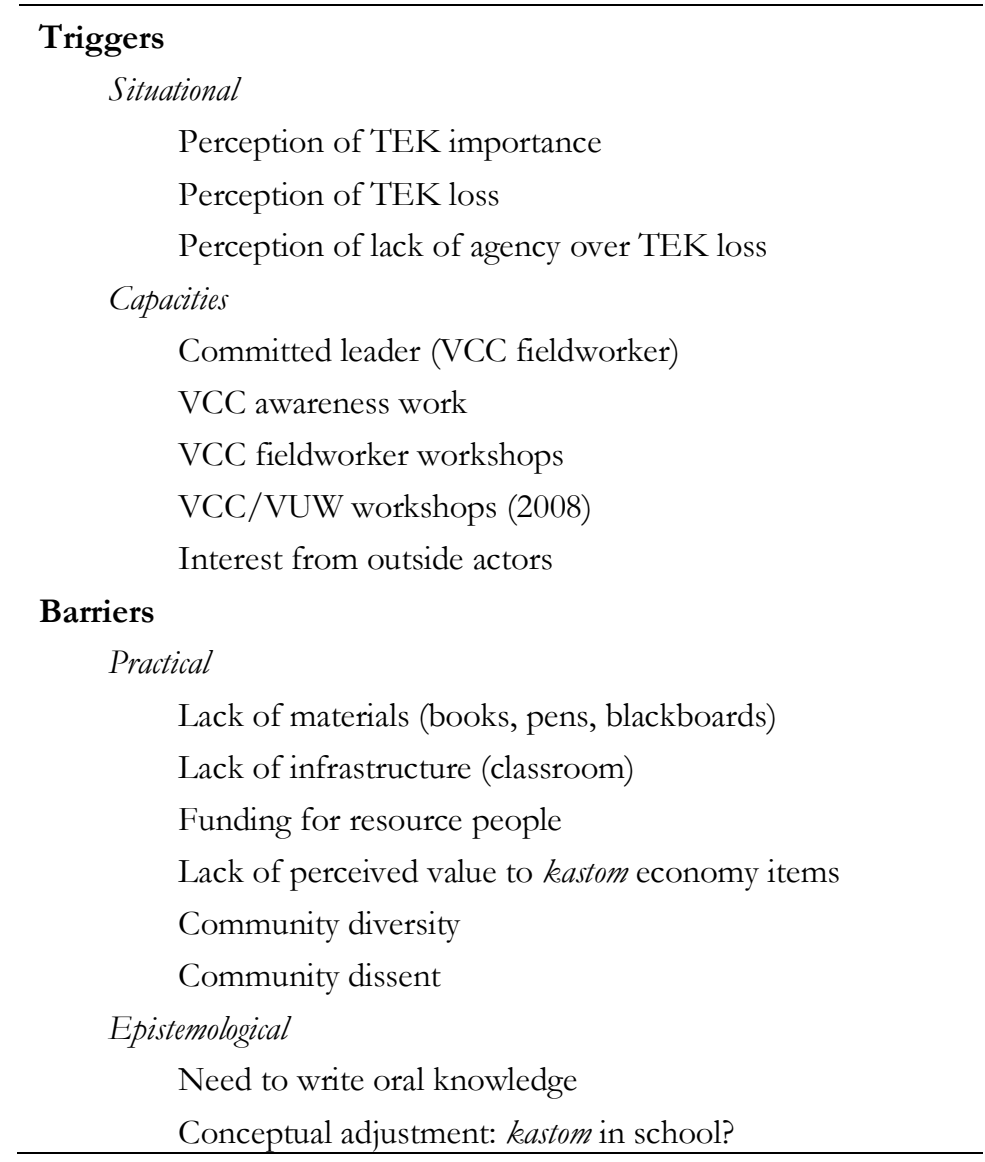

\subsubsection{Triggers}

Participants noted a number of triggers to the formation of the kastom schools. These broadly fall into two key themes, the first discussing situational characteristics of the community at the time of the formation of the schools, and the other regarding specific capacities that allowed the formation of the schools.

Underlying conversations about maintenance of kastom and culture in Vanuatu is a near-ubiquitous perception that TEK (and, indeed, all facets of language and culture) are actively eroding within the 
current generation. All interviewees believed that the younger generation were not as interested or able to learn ethnobiological knowledge, and ascribed this to a number of drivers, including lack of time because of the influence of the formal school system $(n=7)$; a generalised loss of 'respect' for the old ways and old knowledges $(n=3)$; and the influence of the various churches, who at certain times have actively discouraged the transmission of ethnobiological knowledge $(n=3)$.

In the context of the kastom schools, this perception of the erosion of traditional knowledge and culture was discussed as providing the impetus and justification for the creation of separate kastombased education units. Participants often stressed the value of kastom and culture to life in their communities, with five of the 15 individual interviewees noting that kastom was the 'stampa' [trunk] of life. My experience in the field is that the state of kastom and the transmission of TEK and language are highly visible and widely discussed aspects of life within the focus communities. Interviewees felt that these views were widespread amongst the community, noting that "... [the community] want us to do something to preserve the culture and kastom of this village, they are concerned that if we do not all of these things will be lost" (Male, 35). There was then, at least a perception amongst the interviewees that the community approved of actions to mitigate the loss of kastom and culture, and that the widespread awareness of cultural loss throughout the community was an importance necessary condition for the formation of the kastom schools.

Adding weight to the perceptions of cultural loss is a common view of a lack of community agency over the process. This is particularly visible in terms of the formal education system, which ensures that primary and secondary pupils are removed from the village learning context during their formative years (Regenvanu 2005). A founding member of the committee at Unmet noted the effect that this has on the values of the children: "It's my opinion that the kids have to learn about us...even though they go to the formal school they need to know that they are the children of Malekulan people, and that Malekulans have their own kastom and identity..." (Male, c.40).

Therefore, some participants perceived the kastom schools to be a means of reclaiming some measure of control over what children from the village were learning. At Lawa, an interviewee noted that this had a fundamental purpose: “...We have to put things to right, we have to fight for our culture... we don't want our culture to join with the government, to join with the church... we have to start bringing back the warrior culture, it basn't really worked here for a long time" (Male, c.65). This statement has important implications, as it locates the kastom schools as a means of attaining political and ideological independence from the church/state nexus in Port Vila. Under this framing, the kastom schools are not simply a means of ensuring that pupils gain identity as 'man Malekula'; but they are also a conscious effort to define and perpetuate a localised meaning of kastom, which may be in opposition national versions of kastom espoused by the government and church. 
Three of the 15 interviewees specifically noted the need for an intervention in order to increase the transmission of TEK and kastom. Observing the situation in Dixon Reef before the commencement of the kastom school program in 2008, an interviewee remarked: “...the people who know the most, they are sitting over there in a corner. They know it is hard for them to have a place to come and teach the things they know to be true. But if we could have a house, or something like it, I can say that these special people would be able to teach us there" (Female, c.30). This was considered to be crucial, as participants often stressed there were few members of the younger generation who had an interest in connecting and discussing kastom with the older generation. Moreover, they noted that if these connections were not made at an early age, transmission of knowledge became especially difficult: “...if, for example, the children get too old, as old as some of the boys working in the plantation over there, it is too late... they have made their life already, they have already learned other ways" (Male, c.55).

Alongside the situational aspects described above, interviewees also noted several specific capacities or events that led to the formation of the kastom schools. First amongst these was the presence of a committed and able leader, who in each case was a VCC fieldworker. The fieldworkers themselves (n =3) noted the importance of the annual VCC workshops in providing both the information and the technical capability to design programs to revitalise and maintain kastom: “...when I became a member of the fieldworker group, my eyes just opened... I could see that everything they were doing was good. If I hadn't become one of them, I know that I'd never have had the chance to learn about keeping kastom going” (Male, 35). Moreover, the annual workshops provided the space to learn and share ideas, and in particular allowed the spread of information about the various possible methods for maintaining kastom. In point of fact, the fieldworkers from Unmet and Dixon Reef both remarked that it was the example of Lawa, the first school to start, which had pointed them in the direction of a kastom school model.

Other interviewees also noted the importance of a strong leader in the initiation of the kastom schools. In this respect the role of the village chief becomes critical, as noted by members of all three of the kastom school committees. The comments of one female committee member at Unmet were illustrative in this respect:

JM: Do you think that you can teach TEK if there is not a specific organisation for that purpose?

Interviewee: It is possible, but only if the chief arranges it. If the chief does not arrange it here, then it cannot happen.

JM: Why? 
Interviewee: Because we are all around and scattered. We are underneath the chief, and it is his responsibility for making these things happen. So any new programs, or anything like it, it must come from the chief.

(Female, c.50)

Interviewees also highlighted the role of the VCC/VUW workshops, which were attended by seven of the interviewees. The workshops appear to have been important for two major reasons. First, they allowed the space for discussion of ideas. Interviewees who were present at the meetings commented on the importance of being able to air and discuss ideas with peers from other communities: “...the kastom school idea, well that's something where we'll be able to sit down with the elders and discuss the names of things, how to know things... I'd wanted to do something for a long time, however then we went to that meeting on Urupiv [the island where the workshop was held], we were all able to sit down and hear what the others said" (Male, c.50). Clearly, there is a potential for bias here, as I was involved in running the workshops on Urupiv, and it is possible that interviewees intentionally enhanced the importance of the workshop out of either flattery or politeness. However, this response was consistent: of the seven interviewees who had attended the workshop on Urupiv, six noted their critical role in forming the idea of the kastom school.

Second, six interviewees (including some who did not attend the VUW/VCC workshops) also noted that outside interest from VUW in kastom and culture had spurred discussion interest in its maintenance and revitalisation within the community. Whilst this point should not be overstated, given that the vast bulk of work of this nature is undertaken by the VCC, it seems that the simple fact that outside institutions thought that TEK was important enough to come and work on went some way to legitimising the ongoing activities of the fieldworkers for cultural conservation.

\subsubsection{Challenges and barriers}

Interviewees also noted a number of barriers and challenges that are impeding the progress of the kastom schools. These include constraints on the practical ability of the communities to run the schools (such as the presence of infrastructure, materials, and funding), but also include comments on the nature and context of the schools. At their core, this latter group of barriers question the validity of the kastom school idea itself.

The first and most pressing concern of most of the committee members was the lack of basic infrastructure and materials with which to assist in the teaching of kastom and TEK. All participants directly associated with running the skills commented on the need for books, pens, blackboards, and markers: for example, one kastom school leader from Dixon Reef noted in 2008 “... we do need many 
things to start to bring back kastom and traditional knowledge. Right now, the kastom school is ready to go, if we want to build it we can, but first, if we are going to teach, there must be a way of getting books and pens for the children to write in" (Male, c.60). When queried about why materials that are more commonly associated with the formal school system were necessary for facilitating the transmission of kastom and TEK, this response from a young woman teacher from Unmet was illuminating: "... like I said, you white men write things down all the time... whereas before, the elders told all of their kastom stories, our histories, and kept them in their heads... but your head cannot make memory for all time, and when they die, so do the stories... if it is written down it cannot be lost' (Female, c.40). Others, such as the committee meeting at Dixon Reef, noted that the pupils simply do not have 'space in their heads' these days, and after rote learning knowledge at school they needed to write down TEK in order to ensure it was remembered.

The need for writing and teaching materials was also described as stemming from a shift in the value systems of the younger generation. Older participants, in particular those over 70 years of age $(n=3)$ all noted that knowledge that was not written in books was not as well respected, and that there was a fundamental need to transcribe TEK and kastom stories so as to ensure they were on an equal footing with written forms of knowledge. Certainly, written materials are not common on Malekula, and other anthropologists working on Malekula have noted they rapidly become important and politically charged (Curtis 2002; Larcom 1990).

By request of the kastom school committees, one of the outputs of this research was a series of small ethnobotanical resource books, and the kastom school committee at Unmet noted that teaching would not start until these were shipped: “... because we will need to show them [the resource books] to the children, and if they do not know what kind of wood that is, the elders can go and show it to them, and tell them the name that it has" (Male, c.40). These booklets only record a small fraction of the ethnobotanical knowledge in each community, however, and community leaders would clearly like there to be more focus on recording and transcribing oral knowledge.

Underlying concerns about lack materials and housing the kastom school are concerns about lack of funding. On a family-level basis, kastom school levels noted that families had expressed concern that extra money to purchase books and pens for the kastom schools would not be easy to come by, as explained by the Dixon Reef committee “...the problem is, that times are hard a little bit here, because the school fees have gone up. This means that everyone is working too much, they do not have money to spare for extras. We're trying our best to raise funds, but it is hard" (Female, 35).

Another key concern of all interviewees was securing sufficient funding to pay resource people to instruct in the kastom school. This is related to the system of knowledge transmission in pre-contact society, which was graded and based around rank that was accumulated over a lifetime. As such, interviewees commented that some resource people were not comfortable with dispensing 
knowledge for free to a large group of people. Whilst all interviewees noted that you could change the context of knowledge transmission and utilise a kastom school format to teach TEK, there was a need to recompense the elders for their knowledge, in order to align the new contact with previous means of knowledge transmission.

There is a practical dimension to this aspect as well: the cash economy is an increasingly important part of the lives of most Malekulans, and compensation was also required for the time required to prepare and teach lessons in the kastom school. All community members, resource people included, need cash for imported items, school fees, and tithes, and although one of the resource people interviewed noted that he would 'donate' his time, one of the committee members at Lawa ruefully noted: "... if you ask a man to come and do some small project with you, you will hear 'you bave to pay me 1000 vatu for that'. This is one of the things we must struggle with here... one day, they will realise that it is culture and kastom that feed life, not money" (Male, c.65).

The kastom economy (payment through items of customary value, such as yams, mats, and tusks) was to be an integral part of funding the kastom schools as conceived by the VCC fieldworkers. However, interviews reveal that this has not worked out as planned in the initial stages, for two key reasons. First, as indicated by the previous quote, some resource people did not feel that such methods of payment were of sufficient real value, and indicated that they would not give their time if not compensated in cash. Similar initiatives to introduce kastom economy payments for key village institutions such as schools and medical supplies have hit comparable obstacles. Whilst kastom economy items are undoubtedly valued across many spheres of life, in particular in ceremony, for some resource people the opportunity costs associated with teaching in the kastom school meant that only cash payments were appropriate.

As a means of accounting for this, the committee at Dixon Reef had planned to convert kastom economy items to cash. However, their plan was based on a misunderstanding of that the VCC was capable of offering, and is the second key reason why kastom economy payments were not sufficient to run the kastom school. At Dixon Reef, and at two small communities close by, the committee had planned to: "...take the kastom economy items as they come in, then package them and send them to the Cultural Centre in Port Vila. They'll take the kastom economy stuff, convert it into vatu, and send the money back. We'll then use this to pay the teachers of the kastom school' (Male, 40). Other members of the committee at Dixon Reef noted that they had also send a letter to the Director of the VCC asking for funding, and had yet to hear back some six months later. The VCC, whilst it manages large grants for several flagship projects (Section 0), does not have sufficient funds to disburse for such projects, and is reliant on funding from key agencies such as the Christensen Fund. These misconceptions may have had several sources, from simply misunderstanding the rhetoric around the importance of the kastom 
economy, to an understanding the funding obtained by the kastom school in Lawa had been derived from the VCC (when, recall, it emanated from the French Embassy).

While my sample selection was such that wider community dissent to the scheme would not have been picked up, on the whole, interviewees were adamant that the wider community supported their drive to revive the transmission of kastom and TEK. However, one would assume that given the politically charged nature of the topic and the diverse nature of the focus communities, it is likely that some families within the communities would disagree with the intentions or methods of the schools. Indeed, there were indications that this was the case, when both the Dixon Reef and Unmet committee's noted a lack of enthusiasm for their fundraising drives, or when at Lawa a senior resource person noted that the community were not diligent in only speaking the vernacular language as opposed to Bislama: "...this is really important because when the kids come outside, they must use vernacular language outside too. If Mum and Dad do not talk. vernacular language to the kids, it means they will find it hard to pick it up" (Male, 40). However, my conclusions in this regard must remain limited, pending further research with an expanded sample.

One of the most critical barriers to the successful functioning was cited as the fundamental diversity of the communities themselves. Four of the interviewees, all of them elders, noted that in order to effectively transmit kastom and culture, including TEK, pupils and teachers must remain isolated. At Dixon Reef, the original language (Nasarian) is now moribund, and an inland language (Novol) is dominant. One interviewee explained: “... if the TEK and kastom associated with Nasarian is going to be taught back, then the kids will need to stay quietly and go only to the area of Nasarian. That is one way in which we can teach it back. If you mix it with Novol, then you cannot win - the kids, they will only speak. Novol now. That is what is happening today..." (Male, c.70). This is a good example of what is happening in each of the communities, which are conglomerations of multiple family groups, each with their own traditions and kastom, and often their own language. For these interviewees, the natural extension of the previous quote is that TEK can only be transmitted in its true form if each family returns to their ancestral land: "...now, we are all living together in this village, with the church, and now know God and are thinking of going back. Go back to our place. In this place we can teach ourpeople, and other men, we can teach them well... we want to go back and make sure the kastom road, the kastom life that is ours can come back. When we are here inside the community, it is hard...there are so many people here, it confuses us" (Male, c.60).

Finally, several $(n=6)$ interviewees alluded to the underlying conceptual issues inherent within the idea of a 'kastom school'. As one female teacher at Unmet noted, the idea itself is somewhat oxymoronic: "...before, you'd never think about teaching kastom in a classroom - it was just life" (Female, c.40). An interviewee from a fourth community (Tisvel, not included in sample outlined above), noted he was not interested in having a kastom school in his community, as "...all the time you have to spend setting 
one up, the elders are dying. It is better to just pass them a small gratuity and encourage them to gather under the nabanga [fig tree] to talk. That'd be much cheaper..." (Male, c.70).

The paradigmatic shift in the nature of knowledge and transmission from being purely lived experience to that contained in a classroom was clearly problematic, for several reasons. Importantly, one elder at Lawa noted that whilst some types of knowledge (such as language and appropriate familial respect) were appropriate for such a vehicle of transmission, others were not. This included gendered knowledge, or some elements of knowledge that have secret or ritual power and could only be dispensed to those with the appropriate rank and stature to acquire them: “... [exactly what this is] will depend on the elder who is teaching. If we are the same rank, it's ok. But if you're different, them they will be frightened to give out their knowledge...you cannot pass it out to kids who are not old enough to know" (Male, c.40). Moreover, some elements of kastom and TEK are considered to be dangerous, even today: commonly referred to as rabis kastom (rubbish kastom), they include elements of witchcraft and sorcery and are an important part of village life (Rio 2010). Interviews stressed the importance of ensuring that the kastom schools did not inadvertently promote the transmission of the negative aspects of kastom. Clearly, what these items are, and who decides on what should be taught where, is a critical locus for future discussion.

\subsection{Discussion}

A central contention of recent work on TEK maintenance is that in situ models may represent a powerful mechanism for cultural conservation, but this has rarely been tested in the literature (Agrawal 1995; Zent 1999). This chapter has outlined the triggers and barriers to in situ TEK maintenance in the form of 'kastom schools' on Malekula, and in this discussion section I briefly note the triggers to the formation of the schools, before going into some detail on the conceptual and epistemological barriers which appear to be hindering their effectiveness. I also reflect on the appropriateness of the kastom school model for general TEK maintenance. The data presented here provide a useful opportunity to consider the pitfalls inherent in TEK maintenance efforts, and provide a starting point for future discussions on the topic.

\subsubsection{Kastom school triggers}

What lessons, then, can be drawn from the triggers cited by interviewees in Section 8.3.1? First, I note that the establishment of the schools was predicated on a widespread perception and awareness of the importance of TEK throughout the communities. Much of this awareness is likely to stem from the ongoing work of the VCC, which have played a critical role in keeping discussions of kastom, knowledge and power in the forefront of national conversation, particularly through the work of the 
fieldworker program and through regular slots on Radio Vanuatu (Bolton 2003; see Section 4.4). In concert with the widespread perception that knowledge is being lost through the death of elders and resource people (see Curtis 2002), this perception provides fertile ground for the establishment of the kastom schools. This is not to say that there is universal nostalgia for the 'old ways' (for example, the threat posed by rabis kastom is considered to be current and serious), or that perceptions of the benefits of increasing transmission of traditional knowledge are widely shared. However without a widespread awareness of the threatened nature of kastom and culture, ideas such as the kastom schools would have little currency within the focus communities. Other countries would be likely to benefit from the presence of institutions such as the VCC, which may be a precondition for the successful establishment of TEK maintenance efforts.

The second key lesson from the triggers noted by interviewees is that the kastom schools were relatively easily established. The key ingredients (a minimal amount of funding, a committed and informed leader, and space in which to discuss and share ideas) are in no way indicative of a context that is unique to Malekula, and indeed could conceivably arise in a variety of locations. This work also cautiously indicates that with a minimum of extra expense and effort, forums such as the VCC/VUW workshops on Urupiv can be held that can have far reaching benefits. Participatory Action Research approaches aimed at the maintenance of vernacular languages have had success in similar circumstances (Florey 2009), and it seems that research programs may be able to positively contribute the establishment of in situ TEK maintenance programs, if a careful and considered approach is taken.

This research also indicates that the challenges associated with the in situ maintenance and revitalisation of TEK are not insubstantial. Of course, the practical challenges of finding dedicated time, space, and funding to actively transmit traditional knowledge and culture amidst today's social environment (in which the cash economy increasingly demands time commitments, and responsibility for education is centred on the formal system) are significant. These practical considerations are rooted in a series of key debates centred on identity and epistemology.

\subsubsection{The conceptual barriers to the kastom schools}

As a starting point, I observe that the idea of the kastom school represents a conceptual readjustment of the ways in which TEK, and indeed kastom in general, can be transmitted in the focus communities. In adopting the teacher centred, classroom based style of formal education, the kastom schools embody a shift in place of kastom from an entirely lived culture to a discrete body of knowledge that is transmitted in competition to other ways of knowing and being. The data in Chapter Seven have already shown that this can be problematic, and these findings too indicate that 
whilst some interviewees are pragmatic about this shift, others are less so. These informants argued that the best hope for increasing the transmission and integrity of TEK is to isolate pupils away from the sources of distraction, and allow them to live a 'kastom life'.

On a theoretical level, the kastom school concept encourages a shift in the predominant pathway of knowledge transmission from vertical (i.e. from parent to child) to oblique (i.e. from one member of the elder generation to many of the younger) (Cavalli-Sforza and Feldman 1981; Hewlett and CavalliSforza 1986). This has important implications, as vertically transmitted systems of knowledge are hypothesised to be able to sustain more heterogeneous and idiosyncratic knowledge, as knowledge and practice are 'siloed' within individual families (Cavalli-Sforza and Feldman 1981; Reyes-García et al. 2009). This was reflected in interview comments that noted discomfort at the teaching of certain types of knowledge in the kastom school, in particular TEK that is considered to be secret or tabu. Typically, these comments were followed by observations that for such knowledge, special or extra classes would need to be held with only the appropriate recipients present.

A shift from vertical to oblique transmission of knowledge also has important implications for distribution of knowledge, and therefore power, within the focus communities (cf. Agrawal 2002). In vertical systems of knowledge transmission, the primary transmitter of information (the parents) retains control over the process of knowledge acquisition in the learner. However, in systems of oblique or horizontal transmission, knowledge acquisition is dependent on a number of outside sources. As such, the resource people and teachers at the kastom school attain control over knowledge transmission to pupils (just as in the formal school setting described in Chapter Seven). While this may not present a problem for benign knowledge domains, the diverse nature of the communities in question and the presence of secret or restricted information was a source of concern for many interviewees. As noted, however, the research design does not allow me to comment at length on the degree of disagreement within the community.

The dynamics of power within the kastom schools are framed by the issue of gender. The 'traditional' role of women in ni-Vanuatu society is a matter of some debate: where dialogues adopted by some actors past and present (such as missionaries and the National Council of Chiefs) have identified the role of women in kastom is one of 'quiet subservience' (Regenvanu 2005: p 9), others have noted that this represents a contemporary re-interpretation of traditional systems, which were based on a multiplicity of relationships and understandings of reciprocity (Bolton 2003). The idea that 'women have kastom too' is a relatively recent one (stemming from the first meeting of women fieldworkers in Port Vila 1991), and has been an important aspect of the evolution of the concept of kastom itself (Bolton 2003; Tonkinson 1982). However, the fact that the kastom schools are all conceived of, and largely run by, men, gives rise to the concern that they will not adequately account for women's TEK 
and kastom. This bias is rooted in power structures that operate at the local level, and may manifest itself in more time being given to the transmission of 'men's' kastom by male resource people. Potentially, however, such dynamics are a necessary part of local formulation of the kastom school concept.

I argue that the kastom schools represent an evolving conception of the place that TEK and kastom should play in contemporary society. In re-interpreting traditional models of cultural transmission, the key protagonists in the kastom schools have consciously and pragmatically adjusted the meaning of 'kastom' in order to ensure that their version of it is not lost. Such reinterpretations are not surprising, given that kastom is fluid and is enacted differently at local, regional, and national levels (Bolton 2003; Curtis 2002; Jolly 1994: see also Section 4.4). Indeed, kastom in Vanuatu is not thought of as a strict persistence of the traditional, but rather the maintenance of tradition in the changing circumstances of the contemporary world (Jolly 1994). Similarly, the 'tradition' aspect of TEK does not refer to the inflexible transmission of knowledge from the past into the present; rather, it refers to the lens through which new information is interpreted and included into the contemporary context (Berkes 2008). Systems of knowledge, as well is the mechanisms through which they are transmitted, do not develop in isolation and are constantly exposed to new ideas and influences (Bates 2009; Ellen and Harris 2000).

This reinterpretation of kastom and cultural transmission does, however, raise a variety of tensions. One example of these tensions was provided by interviewee comments indicating that the kastom schools would be unable to function without significant outside support. In emphasising that a lack of funding, materials, and resources were key constraints to the functioning of the schools, interviewees noted that, in effect, kastom and culture could not be sustainably maintained without significant input from the outside. To interviewees, this help was either envisaged as grants from various funding agencies or as a means of converting kastom economy items into cash. The lack of ability for the items of traditional value (the kastom economy) to adequately recompense resource people may, as Regenvanu (2005) asserts, be symptomatic of a lingering 'psychology of dependency' whereby "...our own custom [is] inherently flawed or not as good as what comes from the outside" (p 4). Alternatively, it could be simply a realistic comment on the importance of the cash economy in contemporary village life.

Politically, I suggest that the kastom schools can be seen as a conscious effort by the protagonists to realign power in favour of the local community, particularly with regards to education. One of the critical facets of the current formal education system in Vanuatu is its lack of ability to support vernacular language and the transmission of traditional knowledge, for which it has been consistently criticised by ni-Vanuatu scholars (Baeraleo 2004; Niroa 2002; Niroa 2004; Regenvanu 2004; Sanga 
and Niroa 2004). This is mirrored by a large global body of literature that discusses the need for contextualised education curricula, in order to empower locals and improve educational outcomes (Castagno and Brayboy 2008; Thaman 2000). Clearly, interviewees hope that the kastom schools, by appointing local teachers and teaching locally derived subjects, may be able to act as a counterweight to the formal school system. Moreover, they feel that by formalising the teaching of traditional knowledge and practice they may be able to add to the legitimacy of TEK in the eyes of the students. This realignment of power toward the holders of the knowledge is one of the most important aspects of in situ TEK maintenance, and indeed of truly community-based sustainable development in general (Agrawal 1995; Toledo 2001). However, the concept of kastom is enacted a multiple levels (state, regional, and local): there are tensions evident between conceptualisations of kastom as local and unique and kastom as a concept for national unity (Curtis 2002).

\subsubsection{The kastom schools and in situ TEK maintenance}

What, then, is the potential for the kastom school model to maintain and revitalise TEK within the focus communities? As a starting point, I observe that the kastom school's triggers and barriers are context specific, and may only apply to the focus communities on Malekula. However, I would like to comment on the potential for the in situ models such as the kastom schools to maintain TEK, as such discussion will important as ethnobiologists attempt to form a 'community of practice' toward the conservation of biocultural diversity (Maffi and Woodley 2010).

Prima facie, there are several aspects of the kastom schools that may enable them to make a positive contribution towards the maintenance of TEK in the focus communities. Their common focus, for example, on connecting the elder and younger generation in order to facilitate the transmission of cultural knowledge has been identified as a 'key lesson' of successful biocultural conservation programs in Maffi and Woodley's sourcebook (Maffi and Woodley 2010). Likewise, their focus on locally-based institutions (such as the kastom school committees) and on locally-derived sources of authority (such as the expert resource people asked to come in and teach) may enable them to both reinforce current social networks and to be responsive to local needs and desires (Davidson-Hunt 2006). This, in turn, could build social and human capital in each community. Finally, their small and community-specific design enables a focus on local knowledge and language that would be difficult to manage by any other means in the culturally diverse landscape of Malekula Island.

The holistic approach of the kastom schools is an important feature of their design. As noted, ex situ approaches for the conservation of TEK have been criticised for removing the knowledge from the context of its source community, and thereby fundamentally altering the nature and validity of the knowledge itself (Agrawal 1995). In contrast, in situ models such as the kastom schools are instructive 
because they show how the broad cultural context of TEK can be maintained alongside a focus on the empirical knowledge. In focussing first and foremost on the maintenance of kastom and vernacular language, the kastom schools are able to conserve the cultural landscape of knowledge transmission in addition to the specific knowledge itself. There are parallels here with recent discussions in applied linguistics: for example, Fishman's (2001) scale postulates that small and endangered languages are best conserved through the enactment of in situ modules, as it is necessary to ensure the transmission of knowledge in the community environment. Under Fishman's schema, top-down approaches such as formal education are unlikely to succeed unless the language (and in this case TEK) is already being effectively transmitted in the community environment. In situ approaches, may, then, be a means of achieving this (Florey 2009).

There are, however, several key issues that may limit the ability of the kastom schools to conserve TEK. For one, the heterogeneous nature of the focus communities may not be represented in models such as the kastom schools which are necessarily driven by key individual actors with the inclination and ability to do so. On Malekula, the schools were all driven by VCC fieldworkers. However, commentary from Vanuatu has noted that some actors can subvert discussions of kastom to their own end (Regenvanu 2005), and there is no reason to assume in this case that the VCC fieldworkers accurately represent the interests (or the kastom) of the wider community. Similar observations were made by Curtis (2002) when discussing the establishment of the Nahai Cultural Centre on the South Coast of Malekula (2002) “...[the VCC fieldworker concerned was] acting in the name of community, but also raising his own stakes in the game of Melanesian village politics" ( $\mathrm{p}$ 231). Communities are never homogenous social structures (Agrawal and Gibson 1999), and as such approaches to the in situ maintenance of TEK should be mindful that local politics and power dynamics are likely to be at play.

Moreover, the ideas of 'maintenance' and conservation of knowledge may not be endogenous to the communities in question. For example, the 'kastom school' concept does not, in fact, emanate from the focus communities on Malekula. Rather, it has been in circulation in Vanuatu since at least the early 1980's when Vigestad (1984) reported on the formation of kastom schools on the island of Tanna. These schools on Tanna were very different in nature to the kastom schools discussed here, and were in fact versions of colonial formal education re-interpreted by the nascent independence movement (see also Gregory and Gregory 2000). More recently, the VCC has been running its own versions of a kastom school, as part of its national Vanuatu Young Persons Project (Vanuatu Cultural Centre 2005: see Section 0). It seems likely that the VCC fieldworkers in each of the kastom schools on Malekula had heard of the concept from elsewhere, and so brought it with them to the workshops and to the focus communities. The implication is that the kastom school model is not, in fact, adapted to local conditions and may not be appropriate for the maintenance of TEK on Malekula. 
The involvement of researchers is also problematic in the creation of approaches for in situ TEK maintenance. On Malekula, I acknowledge that I, in my position as a foreign researcher, may have inadvertently impacted on the formation of the kastom schools. In co-hosting the workshops in that led to the formation of two of the three schools, and running a research program on TEK within the focus communities, I may have fundamentally spurred the introduction of etic concepts and made the conservation of the VCC's version of kastom desirable within the focus communities. I stress here that my role with the workshops was essentially to provide space for discussion, and at no point did I suggest specific ideas such as a kastom school. However, my evident interest in kastom and TEK may have spurred the development of the kastom school model, which potentially impacted on its suitability at a local scale. As Heckler (2007) has observed in work amongst the Piaroa in Venezuela “...the valuation of particular types of knowledge by global scientists influences the choices local peoples make about what is desirable or even valid in their own paradigms" ( $p$ 91). Future work on in situ TEK maintenance must be aware of this dynamic.

These caveats, then, raise concerns about the ability of the kastom schools (and in situ approaches more broadly) to maintain TEK. First, the kastom schools may not be an appropriate model for the in situ maintenance of TEK. Although the reinterpretation of traditional modes of transmission is one of the key facets of the schools (and a key aspect of community empowerment), in situ may run into similar criticisms as those levelled at the idea of including TEK in formal education: namely, that these, essentially western, modes of knowledge transmission are inappropriate for transmitting the values and norms associated with TEK and kastom. Second, the project-driven nature of the schools, influenced heavily by the fieldworkers, the VCC, and VUW, may mean they are unable to adequately account for community heterogeneity (and in particular, may promote a patriarchal interpretation of kastom). Indeed, they may be more akin to top-down TEK conservation than the idealised bottom-up model. I note here that no development is free of power dynamics, and all initiatives will be influenced by both internal politics and external relationships. These issues may be manifested in different ways in different settings. Third, these case studies problematise the very concept of revitalising TEK, by indicating that the relationship of TEK maintenance to increased social capital is, at best, contestable. Even assuming TEK represented social capital in its original form, it is far from clear that injecting TEK back into the community, in this direct and altered form, would result in a corresponding gain in net social capital.

\subsubsection{Study limitations}

There were some issues with the chosen research methodology. In focussing on interviewing only a small number of the protagonists of the kastom schools, I limited myself to speaking to only those actors who were likely to be positive about their establishment and aims. Moreover, these 
participants were also the most likely to have a stake in the success or otherwise of the kastom schools. Further studies on the kastom schools, or in situ conservation in general, should devote time to broadening the sample with the aim of adding more depth to some of the issues raised in this discussion. The inclusion of local female members in the research team, as adopted for other aspects of this research program (Chapters Five and Six) is likely to be a critical innovation to shed light on the gender dynamics inherent in the kastom school concept. Finally, my chosen methodology was not designed for objectively assessing the success, or otherwise, of the kastom schools. Such studies, including a rigorous discussion of how 'success' would be defined in this context, will no doubt be a valuable contribution in future.

\subsection{Conclusions}

This paper outlines research into the triggers and barriers to the formation of three kastom schools on Malekula Island, Vanuatu. I find that as models of in situ TEK maintenance, the kastom schools have potential to contribute to the maintenance of TEK and kastom in general. Conceptually, however, the kastom schools embody a number of tensions that will need to be negotiated by the key actors and the wider community if they are going to become sustainable over the longer term. Indeed, the lessons gained from this data effectively problematise the concept of TEK maintenance, and emphasise that unless we are clear about what is being conserved, for who, and by who, there is little chance that such projects will have positive outcomes for all concerned. Such findings mirror experiences of in situ conservation within the arena of biodiversity conservation (for example, see Brechin et al. 2002)

In conclusion, I make three final points. First, these data remind us that bodies of cultural knowledge such as TEK are dynamic and constantly changing. Moreover, I note that these changes, on the whole, do not represent passive submission to global and national forces on the part of the community. Rather, they are evident of an active and pragmatic attempt by key actors within the community to redefine knowledge transmission (and indeed the nature of TEK and kastom itself) in line perceived realities of contemporary island life. Such processes were also observed by Curtis (2002), summing up his work on South Malekula: “... [on Malekula] kastom is not so much a nostalgic recuperation of the past, nor even a conservative statement about the power of 'tradition' in the present, but a projection of individual and collective power into the future" ( $p$ 241). This recognition of the dynamism of knowledge contextualises the results from Chapters Five and Six, which presented quantitative data indicative of the same process.

Therefore, approaches for the maintenance of TEK through in situ or other means must be firmly grounded in the source communities from which the TEK emanates. However, like Agrawal and 
Gibson (1999) I caution against the oversimplification of 'community', and note that significant power differentials are always present with regards to the transmission of knowledge and value systems. This is true at national, regional and local levels. With regards to gender, approaches for the revitalisation of TEK should be aware of the gendered nature of TEK and of power relations in the village environment (Agarwal 1992), but also that women are “...not a unitary category, and their environmental relations reflect not only divisions among women but also gender relations and the dynamics of political economies and agroecosystems" (Briggs 2005: p 106). As such, the focus of in situ approaches for TEK maintenance should be on mitigating some of the key power differentials (for example, between the state-run formal education system and local transmission of TEK and culture). Moreover, approaches for the in situ conservation of TEK should be flexible enough to account for the heterogeneity of local knowledge and power structures.

Finally, I note that the complexities of in situ TEK conservation discussed here highlight the need for more targeted research attention toward the maintenance of TEK. The strengthening of social and human capital (and thereby the promotion of social-ecological resilience) is a key task of future ethnobiological research, and the in situ maintenance of TEK may be an effective means of doing this (Zent 1999). If this is the case, then explicit Participatory Action Research methodologies which promote the maintenance of TEK through locally-adapted and emic approaches may be an effective means of maintaining community resilience. However, as in Chapter Seven, the debate around TEK maintenance is politically complex, and involves the negotiation of local power dynamics and heterogeneity. These issues are based in issues concerning local self-determination (Nadasdy 1999), as well as the lived value of kastom in contemporary Malekulan society (Regenvanu 2005). 


\section{Conclusions}

The aim of this thesis was to understand processes of the variation, change and maintenance of TEK on Malekula Island in the Republic of Vanuatu. Throughout the document I have sought to address key gaps in the literature and make theoretical and methodological contributions to guide future research and responses to TEK erosion. To this end, I have presented four core chapters in the form of published or publication-oriented journal articles, each of which makes a unique contribution to the thesis objectives and the literature. The multi-disciplinary and mixed methods approach taken throughout has yielded a diverse range of important insights, which tend to highlight the complexity of patterns of TEK variation and change. These findings will impact on future research and on attempts to maintain or revitalise systems of TEK.

In this concluding chapter I summarise the findings and contributions of this thesis, based on the objectives set out in Chapter One. I then go on to reflect on some of the issues inherent in work within the arena of traditional and indigenous knowledge and the success (or otherwise) of my approach, before suggesting directions for future research based on my data. Finally, I present three cross-cutting themes that emerge from the core chapters of the thesis, before closing the thesis with some brief remarks about the urgency and complexity of the task at hand.

\subsection{Objectives and major contributions}

Each of the core chapters addressed one research objective and made important contributions to the literature. In this section I summarise the major contributions of each chapter, according to the objective it was intended to address.

\subsubsection{Objective one: To assess variation of TEK in four focus communities}

This objective was addressed in Chapter Five, in which I presented the results of a structured interview with 177 participants in four focus communities. The interview collected individual-level data on five measures of TEK (richness and diversity of medicinal plant citations, abundance of reef fish citations, ecological knowledge, and skill proficiency) through the use of freelisting, an ecological 
knowledge quiz, and measurements of skill proficiency. In this chapter I addressed three subobjectives: 1(a), which was to assess TEK variation in relation to socio-demographic explanatory variables; 1(b), which sought to assess evidence of TEK change or erosion; and 1 (c), which was to assess whether different indicators of TEK reveal divergent patterns.

I addressed objective 1(a) in Sections 5.3.1 and 5.3.2. Using a multi-model inferencing approach (Burnham and Anderson 1998), I found that measures of TEK were distinctly patterned by demographic and economic variables. Some of the key results in this regard were that male participants scored more highly than female participants across all measures taken; that scores in most measures were negatively associated with indicators of modernisation, in particular market visitation and linguistic preference; and that formal education was positively associated with some of the measures, including score in ecological knowledge quiz and ability to freelist reef fish species. These findings are important because research to date has indicated mixed and conflicting results regarding TEK variation. For example, previous research has indicated that the formal school associated both positively (Reyes-García et al. 2005) and negatively (Benz et al. 2000) with ethnobotanical knowledge, while the degree of market integration has been shown to have complex impacts on systems of traditional knowledge (Godoy et al. 1998). My findings show that while TEK is patterned via social and demographic variables, the significance of these relationships is variable across communities and between domains of knowledge. As such, these findings extend the current state of knowledge regarding TEK variation, and allow a more detailed analysis of the ways in which it can erode or persist. Moreover, these findings re-iterate that TEK is heterogeneous within and between communities, and should not be conceived as a uniformly distributed body of knowledge (Zent and Maffi 2009).

Objective 1(b) was to assess evidence of TEK change or erosion. To address this objective I analysed the measures of TEK in concert with age of participants and linked these observations with the findings above. I found that there were no associations between the richness of medicinal plant citations and age, but that younger age cohorts cited a more homogenous body of ethnomedical knowledge than the older age cohorts (utilising the Shannon-Weiner diversity index). Interestingly, my data also display a negative association between knowledge of reef fish and age, indicating that knowledge may be increasing in younger generations. This probably stems from the relatively recent establishment of communities on the coast. This association was strongest at Unmet, the most recently-established community. These findings echo others in pointing to some erosion of knowledge (e.g. Lee et al. 2001), specifically indicating that idiosyncratic ethnomedical knowledge of the interior bush environment may be at risk. In concert with data showing variation in knowledge according to market integration and linguistic proficiency, these data indicate that contemporary ecological and social changes may by impacting on knowledge transmission and driving knowledge 
erosion (Quinlan and Quinlan 2007). However, the fact that knowledge of the marine environment appears to be growing amongst the youngest age cohorts highlights the dynamic and adaptive nature of TEK, which can increase under certain circumstances (Guest 2002). These data also emphasised that the salience of different domains of TEK may change according to the broader ecological and cultural context of the TEK holders (Berkes 2008). Therefore, these findings corroborate other commentators in stressing the complexity and dynamism of TEK and the need to move away from simplified and emotive descriptions of TEK loss (Godoy et al. 2009; Zent and Maffi 2009).

Objective 1 (c) was to assess whether different indicators of TEK reveal divergent patterns. To address this objective I compared the five key measures of TEK (in Table 5.20), and found some evidence of divergent patterns, including the positive and negative associations with age noted in the previous paragraph. This finding emphasises that the various domains and dimensions of TEK respond in different ways to social and environmental pressures, and therefore highlights the need for nuanced approaches to measuring TEK variation. Previous studies of this kind have typically focussed on ethnobotanical knowledge, however my results make the important assertion that to gain a more holistic perspective of variation in TEK other domains of knowledge (such as that of insects, fish, or soils) should be taken into account (Reyes-García et al. 2007a).

Overall, the data presented in Chapter Five make three key methodological contributions. First, the literature has thus far been lacking in research that encompasses multiple domains and dimensions of TEK, despite the need for such approaches being a key recommendation of recent reviews (e.g. Reyes-García et al. 2007a). This research filled this gap by sampling different domains (ethnomedical knowledge, ecological knowledge, knowledge of reef fish) and different dimensions (practical skills and theoretical identification and naming) of TEK, and was therefore able to reveal complex patterns of TEK change. Second, this study highlights the need for rigorous and diverse statistical approaches (Begossi 1996). The multi-model inferencing approach employed here allows robust model selection and minimises information loss during analysis. My research is the first to demonstrate its application in the ethnobiological context. Moreover, the use of diversity indices, although not novel (see Begossi 1996; Hanazaki et al. 2000) emphasises the importance of careful and rigorous analysis of data, which are often not analysed to the full extent (for example, Quinlan and Quinlan 2007). In this thesis these techniques allowed the recognition of non-linear trends across age cohorts in the data, and thus the conclusion that knowledge may be homogenising among the youngest age cohorts. Third, the location of this work in Vanuatu is an important contribution: research of this type has been concentrated in particular areas of Central and South America, and despite the status of the Oceania as a hotspot of biocultural diversity there has been little research of this type in the area (Reyes-García et al. 2007a). 
I derive three key policy recommendations from the data presented here. First, ethnomedical knowledge of the interior bush environment on Malekula may be a domain of knowledge that is at specific risk of erosion over the coming decades. As such, the reconnection of traditional pathways for knowledge transmission between elder and younger generations, with a focus on the interior environment, should be a key node of discussion for the maintenance and revitalisation of TEK (cf. Ohmagari and Berkes 1997). Second, the importance of linguistic preference in patterning variation of TEK stresses the critical nature of vernacular language education for the maintenance of TEK. The place of vernacular education in school curricula in Vanuatu is a key site of debate (Siegal 1996), and this research highlights that such developments may be critical for the maintenance of endangered knowledge systems (Zent 1999). Third, changes in TEK systems are complex and adaptive, and different domains of knowledge can show divergent trends. Therefore, programs for the maintenance of TEK should be cognisant of the dynamic nature of culture and seek to clearly differentiate cultural change from TEK erosion (Florey 2009). This will require a detailed understanding of local drivers and complexity (Zent and Maffi 2009).

\subsubsection{Objective two: Cultural transmission of TEK on Malekula}

This objective was addressed in Chapter Six, using an exploratory mixed methods procedure based on both quantitative and qualitative methods (Creswell and Clark 2007). This chapter aimed to fill the literature gap that exists in our understanding of the nature of the cultural transmission of TEK and how this might be changing (Section 6.1). These data contextualised the variation in TEK observed in Chapter Five, and indicated the pathways through which traditional knowledge is acquired and dispersed by TEK holders in the focus communities (Ohmagari and Berkes 1997).

This objective had two parts, the first of which was to describe the cultural transmission of TEK on Malekula. To this end I used a section of the structured interview above $(n=165)$ that examined the participant-reported transmission pathway for 18 TEK skills in five use domains. These data showed that the cultural transmission of TEK on Malekula is largely vertical in nature, and that initial skill transmission generally occurs during the early teenage years at a variety of locations around the village environment. However, transmission pathways were variable between domains, with some food gathering skills predominantly transmitted via horizontal methods, and complex skills such as the carving of ceremonial clubs transmitted through oblique means. There was little consistent sociodemographic variation in TEK transmission pathway, though notably males in the oldest age cohort at Unmet were the only grouping to report learning skills through the nakamal system (the preChristian focus of male social organisation and knowledge transmission). Interviewees who cited a high percentage of oblique transmission events (from non-familial elders) had higher overall proficiency scores, and scored more highly on the key TEK measures from Chapter Five. 
The second part of this objective was to describe changes in systems of cultural transmission on Malekula. This objective was addressed through the use of semi-structured interviews with 18 expert informants, and data were analysed through the use of the Developmental Niche framework (DN: Super and Harkness1986). Using the DN framework, I identified a variety of social, environmental, and individual aspects of change in the cultural transmission of TEK (Table 6.8). These aspects largely coalesced into changes in both the setting (the place of knowledge transmission) and the strategy (the mode of knowledge transmission) of cultural transmission. Participants indicated that the formal school was a driver of both aspects of change: for example, it affects the former by removing children from the traditional learning context, and the latter by being primarily responsible for the introduction of written systems of knowledge. These changes in transmission systems were perceived by interviewees to be impacting on the ability of novices to acquire TEK from knowledge holders.

Both sets of data contribute to knowledge around the cultural transmission of TEK. This is an under-represented research area within ethnobiology, and a critical literature gap to fill given the importance of processes of cultural transmission in the persistence and erosion of TEK (Zarger and Stepp 2004). The quantitative data extends our knowledge of how TEK is transmitted through social networks on Malekula. These findings dovetail with other ethnobiological research based in different settings (e.g. Eyssartier et al. 2008; Lozada et al. 2006; Ohmagari and Berkes 1997), and appear to add weight to the contention that vertical transmission is often a formative pathway for the acquisition of TEK in the early teenage years (Hewlett and Cavalli-Sforza 1986). These findings also indicate that oblique TEK transmission is important for learning other aspects of TEK (such as ethnomedical knowledge), which highlights the critical role of elder resource people in dispersing environmental knowledge (cf. Reyes-García et al. 2009). The qualitative data strongly suggest that cultural transmission on Malekula has changed in recent years, and that changes in the social and ecological environments have been mirrored by changes in systems of knowledge transmission, which may in turn influence importance and salience of different domains and dimensions of TEK (Greenfield et al. 2000).

Overall, these data offer three unique contributions. First, no quantitative studies thus far have analysed changes in systems of cultural transmission of TEK, despite this being a critical aspect of the persistence and erosion of knowledge (Ross 2002a). In highlighting the setting and strategy for the cultural transmission of TEK as key nodes of change, these data provide a focus for efforts to support systems of TEK in the focus communities, and guidance for similar programs elsewhere (Zent 1999). Second, no ethnobiological studies thus far have used an explicitly mixed-method approach to studying cultural transmission. This is important, as quantitative and qualitative methodologies can derive differing results regarding cultural transmission (Zent and Maffi 2009), and 
so should be used in combination to maximise the strengths (for example, the ability to garner large sample sizes using quantitative methods) and minimise the weaknesses (for example, the tendency of quantitative methods to approach complex systems in a reductionist manner) of each approach (Creswell and Plano Clark 2007). Third, no studies thus far have linked research into the variation of TEK with changes in the modes of cultural transmission of that knowledge. This is critical, as processes of cultural transmission govern the erosion or persistence of knowledge and are likely to underlie observed patterns of variation (Ross 2002b). The data presented in Chapter Six add value to the findings in Chapter Five by suggesting that changes in cultural transmission are a mechanism for the observed variation (and inferred change) of TEK on Malekula.

Two key policy recommendations stem from Chapter Six. First, traditional lines of cultural transmission between parents, elders, and children should be a focus for those seeking to conserve at-risk systems of knowledge (Bates 2009). However, such efforts should be cognisant that systems of cultural transmission are responsive and adaptive to new ecological and social conditions (Greenfield et al. 2000), and that new forms of transmission (i.e. within peer groups) may be important for the maintenance (or even growth) of some domains of TEK, such as that of the marine environment. Second, interventions to support the transmission of TEK must pay attention to both the setting and the strategy for knowledge transmission, as these were identified as key nodes of intergenerational change within the focus communities. In particular, the formal school system was identified as impacting on knowledge transmission, thus supporting other data from Vanuatu and the Pacific which indicates formal schooling may be detrimental to the transmission of TEK and kastom (Pene et al. 2002; Sanga et al. 2004; Thaman 2000). These issues were explored more thoroughly in Chapter Seven and Eight.

\subsubsection{Objective 3(a): To assess perceptions of the value of TEK to formal school curricula}

I addressed this objective in Chapter Seven (see also McCarter and Gavin 2011). In that chapter, I aimed to assess the potential for formal education systems in Vanuatu to contribute to the maintenance and revitalisation of TEK, and the potential value that TEK may be able to add to formal school curricula. I gathered data using a series of semi-structured interviews with three groups of informants: school teachers on Malekula; TEK experts on Malekula; and officials within the Vanuatu Ministry of Education (total $\mathrm{n}=49$ ).

Most interviewees believed that if an appropriate format could be developed, TEK could be included in the formal education system. They noted that approaches such as these have potential to maintain customary knowledge and practice in the focus communities. Participants identified several specific 
domains of TEK for inclusion in school curricula, including ethnomedical knowledge, agricultural knowledge and practice, and the reinforcement of respect for traditional authority and values. If such an approach were to be taken, interviewees noted that TEK has potential to add value to formal education systems in Vanuatu by contextualising the content and process of curricular delivery, and by facilitating character development and self-awareness in students. These benefits corroborate those highlighted in recent literature (Castagno and Brayboy 2008; Ruiz-Mallen et al. 2010) and with UNESCO-mandated goals for curricular reform (Delors 1996). Such approaches may also assist in the maintenance and revitalisation of at-risk systems of ethnobiological knowledge. However, interviewees also noted a number of practical and epistemological barriers to teaching TEK in school. These included the cultural diversity of Malekula, tensions between public and private forms of knowledge, and multiple values of TEK within the community.

The data presented in this chapter make three primary contributions. First, discussion of the validity of the various approaches to TEK maintenance has been surprisingly sparse in the literature (cf. Agrawal 1995), and these data represent an important contribution. In particular, several commentators have recommended that formal schooling be used as a mechanism for TEK conservation (e.g. Heckler 2007; Zent and Zent 2004), but few have discussed it in detail or presented work to outline how such proposals might operate on the ground. Given the potential for negative consequences suggested in these results, I suggest that there is need for caution and further research around the use of top-down mechanisms such as formal schooling in the maintenance in TEK. Second, in suggesting specific ways in which TEK might be incorporated into school curricula, these interview results provide a starting point for the discussion of contextualising education using TEK in Vanuatu. The importance of local knowledge for education in Vanuatu has been recognised recently by Vanuatu's Ministry of Education (Vanuatu Ministry of Education 2010) and the Vanuatu Cultural Centre (Baeraleo 2010), but there is still more work needed for the effective integration of TEK into formal education. This is typical of many areas of the world, where epistemological diversity is often marginalised by formal schooling (De Sousa Santos 2007), which therefore fails to take account of TEK and other traditional knowledge systems (Kimmerer 2002). Third, these data indicate that formal education systems may be unable to account for the full cultural landscape of traditional knowledge systems. Importantly, they may obscure traditional settings and strategies for knowledge transmission, which were revealed to be critical nodes of intergenerational change in Chapter Six. Consequently, formal school systems are unlikely to be sufficient for the maintenance of entire bodies of TEK, and additional approaches (such as in situ TEK conservation) may be needed (Zent 1999). 


\subsubsection{Objective 3(b): To assess community perceptions of in situ TEK conservation}

This objective was addressed in Chapter Eight. In this final chapter, I aimed to outline the triggers and barriers to the formation of three kastom schools, local organisations for the in situ maintenance of TEK on Malekula. I gathered data from key protagonists within the kastom schools, using semistructured interviews $(\mathrm{n}=15)$ as well as focus groups with kastom school committees $(\mathrm{n}=4)$.

The findings presented in this chapter indicate that the kastom schools have potential as a means of in situ TEK conservation. In particular, such approaches have the potential to re-open pathways for cultural transmission between elder experts and pupils, as such mitigating some of the changes in cultural transmission that have been noted in the previous chapters. Such approaches are congruent with those suggested by authors working on the revitalisation of vernacular language, which have emphasised the role that community-based institutions for knowledge transmission must play in the revitalisation of at-risk cultural systems (Florey 2009; Romaine 2008).

However, the kastom school model also embodies a number of tensions, particularly surrounding the ways in which kastom is interpreted in the contemporary environment. Interviews revealed that the kastom schools represented an active reinterpretation on the part of the protagonists of the ways in which TEK and kastom are lived, taught, and transmitted. Issues of power, particularly surrounding who controls and teaches the subject material, are thus critical in the culturally diverse environment of Malekula (cf. Agrawal 2002). These conceptual issues, as well as practical barriers such as lack of space and funding, are currently impacting on the ability of the kastom schools to function effectively, and may fundamentally undermine the ability of such models to maintain TEK. The issues described in this chapter draw attention to the fact that that TEK 'maintenance' involves much more than the simple conservation of static knowledge and practice. Indeed, the debate is politically broad and involves the negotiation of local power dynamics and heterogeneity, rooted in issues surrounding indigenous self-determination (cf. Nadasdy 1999) and the lived value of kastom in contemporary Malekulan society (Regenvanu 2005).

The contributions of these data are threefold. First, the data presented in this chapter indicate that in situ schemes may be relatively easy and cost effective to establish, and offer the potential for the conservation of the entire cultural landscape of TEK by re-establishing lines of transmission within the communities. Moreover, the practical barriers to their functioning (such as a lack of dedicated space and funds) may be relatively easily overcome, and could be potentially assisted by dedicated research utilising Participatory Action Research methodologies. Such approaches would be consistent with researcher obligations under the International Society of Ethnobiology's Code of Ethics.

Second, however, these data raise a host of broader issues that highlight the situated nature of TEK 
and kastom, which is enacted in a number of ways throughout the community. Therefore, the findings in this chapter serve to problematise simplistic accounts of TEK maintenance, and draw attention to the wider political issues inherent in the 'conservation' of any aspect of a worldview(Agrawal 1995; Zent 1999). Third, and overall, discussions around the maintenance of TEK have thus far been sparse in the literature (Zent 1999). Given that in situ means have been promoted as the most appropriate approach to TEK maintenance (Agrawal 1995), the data presented here are an important addition to knowledge around the theoretical and practical issues inherent in the concept.

\subsection{Reflections}

As in any research program, there are a number of aspects of this thesis that warrant discussion and reflection. In this section I reflect on how some of the issues regarding TEK research that were raised in the introduction have impacted on this work. I'd note that specific methodological issues have been mentioned in the chapters throughout the thesis. I acknowledge that some of these, such as the lack of longitudinal (time-series) data and significant gender biases in semi-structured interview samples, are significant and have influenced the outcomes of this research. I will not, however, repeat myself by listing each of them again.

In the introductory chapters (Sections 2.3 and 3.2) I noted several key issues that had the potential to undermine the validity of this research. The most important of these were issues surrounding power dynamics and representation of indigenous peoples, and the significance of gender in this research. Critiques included the difficulty of representing lived and dynamic indigenous knowledge in text (Brosius 1997); the fundamental issues with delineating and specifying 'indigenous' knowledge in opposition to other forms of knowledge (Agrawal 1995); and the power dynamics that are inherent in any research relationship, especially between western academics and indigenous people or between male researchers and female participants (Scheyvens and Storey 2003; Smith 2005).

Undoubtedly my positionality as a male, scientifically-trained, outsider has fundamentally impacted on the nature of this thesis and the findings presented herein. Indeed, my interpretation of TEK and analysis through the lens of an empirical study may be unrecognisable to the TEK holders with which I spoke, and if another researcher with a different positionality (say, a ni-Vanuatu woman) was to conduct similar research they may find different results. That said, the mixed methods research design, the rigorous ethical procedure (Section 3.3), and the high degree of input from the VCC and fieldworkers have worked to reduce the power imbalance inherent in research relationships and promote a sensitive discussion of the data. I'd also note that the fact I am likely to have interpreted aspects of TEK differently to the TEK holders themselves is not in itself a fundamental problem: the purpose of this research was not to record TEK per se, but rather to look for patterns and discuss 
change and maintenance of traditional knowledge. In situations where I was actively recording TEK - such as the design of ethnobotanical resource books for use in the kastom schools (Appendix 11.13) - I attempted to ensure expert participation to the highest degree to increase accuracy.

However, as noted in Chapter Eight, the recording of knowledge in this form was a matter of active debate within the focus communities. Indeed, there were (and remain) tensions regarding the transcription and writing down of TEK on Malekula throughout the research. The act of writing information down is a fundamentally political process that is consciously guided and deployed by the actors concerned for various purposes (Curtis 2002). For example, on Malekula the texts of early anthropologists John Layard and Bernard Deacon have become important arbiters of 'truth' in disputes over land and resources, some 70-80 years after they were first produced (Geismar 2009; Larcom 1990). As Curtis (2002) writes of his own work in South Malekula, recounting an incident in which one community member was concerned that he had recorded an incorrect story: “... [this], however, was a totally valid concern: the fact that writing down a story can then give it local authority. Although I assiduously tried to avoid giving such authority to my work, no doubt one day, somewhere on Malekula, people will use my texts for a dispute" ( $\mathrm{p} 217)$. Because summaries of my work will be sent to the communities, in addition to the ethnobotanical resource books, my work faces similar problems. I attempted to mitigate the politicisation of my work by thoroughly checking information with a variety of informants, careful informant selection, and a focus on noncontroversial domains (most notably, not land). However, like Curtis, I acknowledge that the printed outputs of this work may be for purposes contrary to their original intention in the future.

Another issue, particularly evident in the early stages of the research, was tension over the conceptualisation of TEK. This largely arose because the direct translation into Bislama provided to me by the VCC ('kastom save blong envaeromen') proved to be understood in a number of ways by interviewees. This is likely to be the result of several intersecting factors, not least my limited proficiency in Bislama during the scoping study (Section 4.9), the multiple meanings of kastom understood throughout the community, and the difference between urban and rural versions of Bislama (Crowley 2003). However, it is also likely to derive from the fact that the designation of 'TEK' as a separate entity from wider 'culture' is a distinctly academic, western, idea. To interviewees, the environmental aspects of kastom are inseparable from the wider cultural framework in which it is embedded. This is, of course, the point that Berkes (2008) was attempting to reinforce with the nested egg diagram (Figure 2.1). However it contrasts with common portrayals of TEK in the academic literature, which seldom have the means to consider the wider aspects of TEK in a particular survey. I overcame this difficulty by referring to the specific aspects of kastom I was referring to, such as ethnomedical knowledge, because this was a locally salient and distinct domain 
of knowledge that made sense in discussion. However, I'd argue that the embedded nature of TEK needs to be more commonly recognised and deployed in field research.

\subsection{Further research}

This research made a number of important contributions, which should be replicated in future studies in order to continue to fill the literature gaps outlined in Section 2.7. In particular, future research should take a broad approach to measuring TEK variation (Zent and Maffi 2009), and should focus on elucidating patterns in other domains of TEK, such as reef fish and insects.

Moreover, future studies should utilise the most rigorous quantitative data analysis methods (such as multi-model inferencing) and mixed methods approaches to understanding complex patterns of change and dynamism. Finally, and most critically, upcoming research should continue to close the literature gap surrounding the TEK maintenance and revitalisation efforts, in order to begin to answer some of the critical epistemological issues raised throughout this thesis.

Other future research areas extend from the findings presented in this thesis. One of the most urgent areas of future research surrounds the links between vernacular language and TEK. It would be instructive to clarify the relationship between language acquisition and TEK transmission, and it will be critical to maintain discussion around the mechanisms through which both can be maintained. This is especially urgent in culturally diverse environments such as Malekula (where, recall, up to 15 languages are moribund), and points to the need for cross-disciplinary links between applied linguistics and ethnobiology. As noted by others (e.g. Godoy et al. 2009), longitudinal studies of TEK will also be important in order to understand more precisely how TEK changes in response to indicators of modernisation. I'd note, however, that it is essential that these studies contain a component that explores temporal changes in systems of cultural transmission: my work is rare in focussing on this aspect, and without more work in this area it will be difficult to specifically identify the mechanisms that underpin variation and erosion of knowledge. Moreover, such studies must be founded on a nuanced understanding of local context: for example, in Melanesia clan membership is key variable that structures knowledge throughout the population (Sanga, pers. comm.; May 2012), but has never been formally incorporated into of models of knowledge variation and erosion. Finally, at a global scale, it will be important to systematically examine the relationship between TEK and the resilience of socio-ecological systems. Most of the evidence presented so far, including that cited in support of the ideas herein, is theoretical in nature. Identifying the precise mechanisms through which diverse systems of TEK can promote resilience, or ameliorate system vulnerability, would be a powerful argument in favour of continued efforts to maintain and revitalise TEK. 


\subsection{Cross-cutting themes}

In this final section, I discuss three cross-cutting themes that underpin and intersect with all or some of the chapters.

\subsubsection{The value and dynamism of TEK on Malekula}

Throughout this thesis I have emphasised the value of TEK and kastom to everyday life on Malekula. As noted in the introduction and throughout, Vanuatu is one of the locations in the Pacific, and the world, in which cultural traditions have been retained to the greatest extent (Regenvanu 2005). Although the purpose here has not explicitly been to document TEK per se, evidence of the depth and value of local knowledge on Malekula has accumulated throughout the thesis. Two examples include the high richness of medicinal plant citations in Chapter Two, which potentially decrease reliance on the under-resourced western medical system; and the potential for TEK to contextualise and add value to formal education curricula in Chapter Seven, which indicate that TEK could play a role in increasing the educational performance of the formal school system. In addition, TEK is an intrinsic facet of the cultural diversity of Malekula: framed by the variety of spoken languages in which it is encoded, it is deeply implicated in the formation of identity and values of Malekulans as 'man ples' [local] (Hickey 2005).

It is likely that these and other aspects of TEK on Malekula are linked to the resilience of the socioecological systems in which the focus communities are situated. In this case, ethnomedical knowledge could be considered a store of human capital, and the social networks inherent in systems of TEK transmission and use may represent social capital (Pretty 2011). Social and human capital, in concert with natural and financial capital, influence the resilience of a given socio-ecological system; the erosion of some of these forms of capital may increase overall vulnerability (Folke et al. 2007). TEK systems can increase the resilience and lower the vulnerability of rural communities in the face of social and ecological challenges, as shown by research elsewhere in Vanuatu (e.g. Campbell 2006; Warrick 2009) and globally (e.g. Mercer et al. 2007). However, as I will indicate below, the role of TEK in supporting social capital is likely to be variable across time and space.

This thesis also provides evidence of the erosion of TEK on Malekula, which can be drawn from all the data sources presented throughout the core chapters. Semi-structured interviews revealed a nearuniversal perception of TEK loss amongst community members, driven by a nexus of factors associated with the changing social and environmental conditions. Quantitative data (Chapter Five) supported these assertions, and indicated that individual level of TEK in the focus communities is strongly associated with indicators of modernisation (such as linguistic preference) and may be 
homogenising amongst younger age cohorts. Data in Chapter Six indicated a mechanism for the observed variation of TEK amongst the sample population, and suggested that the strategies and setting for cultural transmission are changing and may be less appropriate for the transmission of TEK to younger age cohorts. The erosion of TEK amid contemporary social and ecological change has been anticipated by a number of authors worldwide (e.g. Cox 2000; Quinlan and Quinlan 2007). The data presented here corroborate some of these suggestions, and indicate that historical factors (such as geographic shifts from the interior environment to the coast) and the ongoing permeation of western ideas may be negatively impacting on the transmission of TEK.

That said, one of the outstanding features of this research is the temporal and spatial complexity of processes of TEK change. Systems of TEK are adaptive and are tightly linked to ecological and social context in which the TEK is generated, transmitted, and lived (Greenfield et al. 2000). This research clearly demonstrates that while ecological and social change means that some information may be at risk of erosion from within a social network (for example, ethnomedical knowledge of interior Malekula) other information may be gained (such as knowledge of the marine environments). Systems of cultural transmission are actively reinterpreted by key actors as social circumstances change, resulting in differences in the kinds of knowledge that are salient within the community (Greenfield et al. 2000). Moreover, the drivers of this ecological and social change are temporally and spatially variable, and will result in localised changes to TEK systems and localised impacts in terms of the resilience of the social-ecological system (Anderies et al. 2004). Therefore, these data reinforce our understanding of TEK and social-ecological systems as fundamentally adaptive (Berkes et al. 2000), and emphasise the inextricable links between biological and cultural diversity (Maffi 2005).

The dynamism that characterises biocultural diversity in Vanuatu should influence discussions around the use and promotion of TEK. Some of the literature based in Vanuatu has emphasised the importance of biocultural approaches to resource management, in particular the need to recognise, acknowledge, and strengthen TEK systems (Hickey 2005). However the data presented here emphasise that TEK is spatially variable and is contingent on historical factors: for example, whilst there is no doubt that groups with a history of living on small islands (such as on Urupiv Island) are likely to have a detailed and comprehensive knowledge of the reef environment, this is not be true of the relatively recently settled coastal areas over much of the island. Moreover, the argument that promotion of TEK seamlessly increases social capital is also contentious, as indicated by some of the potential negative outcomes of TEK maintenance identified in Chapter Seven and Eight (such as the promotion of 'bad' kastom, and the infringement on educational outcomes). Whilst, in some areas, strengthened systems of cultural transmission of kastom are likely to increase the ability of groups to affect collective action, and thus support social capital, this may not be the case in all areas and for all sectors of the community. Biocultural conservation approaches, then, must be founded on a nuanced 
understanding of local history and complexity, as well as recognition that systems of cultural transmission are adaptive and dynamic.

\subsubsection{The 'maintenance' of TEK}

TEK is clearly a critical resource, and the imperative to record, document, and maintain endangered knowledge systems should be a key focus for ethnobiologists over coming decades (Zent 1999). Indeed, several authors have called for a more in-depth focus on the maintenance of TEK systems, and have proposed a number of potential methodologies for achieving this aim (see Section 2.6). However, little research has explored the validity and salience of these options, two of which were explored in Chapters Seven and Eight. These data, whilst seeking to recommend optimal courses for future action, indicated that the maintenance or conservation of TEK is an uncomfortable proposition.

One issue is that a focus on particular aspects of TEK (such as ethnomedical knowledge) without attention to the underlying cultural framework or methods of transmission is unlikely to be successful (Bates 2009). In Vanuatu, this means that discussions of TEK maintenance and revitalisation return inexorably to kastom and its place in contemporary society (Section 4.4). The revival of traditional culture, whilst promoted vociferously by the VCC and fieldworkers in the village environment, hinge around a multiplicity of understandings of the nature of kastom (Curtis 2002). Chiefs and elders (who are commonly key TEK holders) may espouse a version of kastom that might be particularly uninspiring for women and for younger people, and which may owe more to recent political struggles against the colonial condominium government than to centuries-old tradition (Regenvanu 2005). Similarly, parents, teachers, and policy makers may have their own version of kastom to perpetuate, and their own reasons for seeking the revitalisation of TEK within their communities (Curtis 2002). Each of these parties may seek to pragmatically and consciously alter pathways for cultural transmission, such as the kastom schools discussed in Chapter Eight.

Approaches to strengthen the transmission of TEK must, by nature, pass some educational authority to the TEK holders and experts themselves (Agrawal 1995). However, such approaches must be cognisant of intra-community power dynamics, which are based in the shifting identities of community participants and of kastom itself (Smith 2005).

In stressing the complexity of TEK change, however, this research calls into question the ability of programs that seek the 'maintenance' or 'conservation' of knowledge to adequately account for local heterogeneity. Knowledge and power are unevenly distributed throughout the community (a dynamic that is amplified in the culturally diverse environment of Malekula), and thus questions of the 'maintenance' or 'conservation' of knowledge immediately beg the question of which knowledge is to 
be conserved, by who, and for who (Agrawal and Gibson 1999). The value of cultural continuity against local adaptability will be different to different actors in the community, and this will fundamentally impact on the domains and dimensions of knowledge that are 'maintained' through TEK conservation programs (Heckler 2007). In this sense, Nandini Sundar's (2002) admonishment that educational projects are always “...inflected by race, class, or gendered assumptions... [and] that pedagogy...involve[s] projects of moral transformation" ( $\mathrm{p}$ 374) seems particularly salient, and may be enacted through power dynamics that exist at a local level - for example, between TEK experts and youth, or between men and women (Briggs 2005). Such acknowledgements indicate that in situ TEK conservation should not be seen as a panacea solution to the erosion of traditional knowledge and practice, and that such approaches may serve to obscure heterogeneity and power dynamics at the local level (Nadasdy 1999). This does not, of course, mean that such approaches should not be considered in the future. However, such dynamics are likely to be at play in various contexts around the world, and will be amplified in culturally diverse nations such as Vanuatu.

\subsubsection{Multiple processes, multiple approaches}

The epistemological issues outlined above should not obscure the urgency of TEK maintenance efforts. Although caution is needed in the way that such programs are set up, the ongoing homogenisation of knowledge and the advanced age of resource people in these communities mean that on Malekula, as elsewhere in the world, TEK maintenance should be a priority for research and practice (Zent 1999). However, the data presented throughout this thesis do call attention to the need for more nuanced and holistic discussion in ethnobiology around TEK maintenance. There has thus far been little discussion about how TEK conservation should proceed, despite the presence of multiple epistemological and practical challenges and despite several calls for more discussion in the literature (Ohmagari and Berkes 1997).

I suggest that some of the most trenchant issues outlined above may be circumvented by reorientating the discussion of TEK maintenance around educational concepts such as 'lifelong learning' (e.g. Sanga 2002) and the latter two pillars of the Delors Report ('learning to know' and 'learning to be': see Section 7.1). These approaches emphasise that basic education should prepare pupils by not only passing information, but by also passing self-awareness and the ability to live connected, informed and community-oriented lives (Taufe'ulungaki 2002). In framing the discussion in these terms, the focus is shifted away from the 'maintenance' of decontextualised pieces of knowledge, and instead moves toward a holistic discussion of desired outcomes of pedagogy and tutelage in the formative years (Thaman 2000). Such moves may require a re-distribution of power (cf. the 'democratisation of knowledge systems' suggested by Shiva 2006), such that local communities are able to determine the kinds of knowledge and means of cultural transmission that 
are appropriate in their particular social-ecological context (Zent 1999). TEK maintenance, then, would become fundamentally linked to moves to facilitate the self-determination of indigenous peoples (Nadasdy 1999), and would ensure that TEK continues to adapt and persist into the future.

More broadly, I argue that the complexity of the issues discussed throughout this thesis highlight the need for holistic and multi-pronged responses to biocultural homogenisation. These responses may be necessarily issues-based: for example, if improving marine resource management at Unmet was the goal, there may be a need for science-based education around marine ecology and information (c.f. Foale 2006b), as well as support and recognition of the traditional structures of authority in the community environment. If, on the other hand, the goal is facilitating the maintenance of traditional ethnomedical knowledge, a variety of approaches that may include the utilisation of formal school curricula, linguistic revival, and community-based initiatives are likely to be needed. All responses, however, must seek to build on nuanced and sensitive understanding of local history and complexity. Indeed, failure to do this may result not only in the incomplete attainment of goals, but in irrevocable damage to the TEK systems themselves.

\subsection{Conclusion}

In this thesis I have presented four core chapters that examine the variation, transmission, and maintenance of TEK on Malekula Island, Vanuatu. The results presented throughout show that recent changes in the geographic and cultural setting of the focus communities have affected the inter-generational transmission of TEK. This has led to the the homogenisation of some knowledge domains and vigorous debate about the place of kastom in contemporary community life. These data highlight the rapid adaptation and resilience of TEK systems, and show that methods of cultural transmission are actively adapted to suit changing environmental and social conditions. My findings also emphasise the multi-dimensional and complex nature of TEK, which is patterned throughout the community and is situated within a wider cultural and political framework that belies simplistic empirical explanation or attempts at static maintenance. In sum, this thesis highlights the importance and fragility of TEK systems, at the same time as calling attention to the complex nature of TEK change and arguing for nuanced, sensitive, and adaptive approaches to its maintenance.

I began this thesis by noting the urgency of the challenges facing many areas of the world, and by proposing that indigenous knowledge systems will be critical aspects of any response to global environmental and social issues. Throughout this thesis I have advanced our understanding of the ways in which TEK systems change, adapt, and are maintained in the diverse and dynamic environment of rural Malekula. What is now needed is sustained and detailed work on traditional knowledge in Vanuatu, and in other contexts, so as to enable the maintenance of social capital and 
resilience in social-ecological systems. That TEK will be a critical aspect of a sustainable future, both globally and in Vanuatu, is not under debate. However which aspects of TEK are emphasised, how they evolve under shifting social and ecological conditions, and how issues of power and heterogeneity are negotiated will remain critical nodes of discussion over the coming decades. 


\section{References}

Adger, N. W. (2003). Social capital, collective action, and adaptation to climate change. Economic Geography, 79(4), 387-404.

Agarwal, B. (1992). The gender and environment debate: Lessons from India. Feminist Studies, 18(1), 119158.

Agrawal, A. (1995). Indigenous and scientific knowledge: Some critical comments. Indigenous Knowledge Monitor, 3(3).

Agrawal, A. (2002). Indigenous knowledge and the politics of classification. International Social Science Journal, 54(173), 287-298.

Agrawal, A. (2009). Why indigenous knowledge? Journal of the Royal Society of New Zealand, 39(4), 157-158.

Agrawal, A., \& Gibson, C. G. (1999). Enchantment and disenchantment: The role of community and natural resource conservation. World Development, 27(4), 629-649.

Aikenhead, G. (1993). Foreword: Multicultural issues and perspectives on science education. Science Education, 77(6), 659-660.

Aikenhead, G. (2001). Integrating western and aboriginal: Cross-cultural science teaching. Research in Science Education, 31(3), 337-357.

Aikenhead, G., \& Ogawa, M. (2007). Indigenous knowledge and science revisited. Cultural Studies of Science Education, 2, 539-620.

Akerreta, S., Cavero, R. Y., \& Calvo, M. I. (2007). First comprehensive contribution to the medical ethnobotany of Western Pyrenees. Journal of Ethnobiology and Ethnomedicine, 3(26).

Alcorn, J., \& Toledo, V. M. (1998). Resilient resource management in Mexico's forest ecosystems: The contribution of property rights. In F. Berkes, C. Folke \& J. Colding (Eds.), Linking social and ecological systems: Management practices and social mechanisms for building resilience. Cambridge: Cambridge University Press.

Alexander, C., Bynum, N., Johnson, E., King, U., Mustonen, T., Neofotis, P., et al. (2011). Linking indigenous and scientific knowledge of climate change. Bioscience 61(6), 477-484.

Allen, G., \& Steene, R. (1994). Indo-Pacific Coral Reef Field Guide. Singapore: Tropical Reef Research. 
Altieri, M., \& Merrick, L. (1987). In situ conservation of crop genetic resources through maintenance of traditional farming systems. Economic Botany, 41(1), 86-96.

Anderies, J. M., Janssen, M., \& Ostrom, E. (2004). A framework to analyze the robustness of socialecological systems from an institutional perspective. Ecology and Society, 9(1), 18-35.

Andresen, P. (2001). Gender and indigenous knowledge: Experience from Nigeria and the USA. Indigenous Knowledge and Development Monitor. Retrieved from http://www.nuffic.nl/ciran/ikdm/9$1 /$ andres.html

Apaza, L., Godoy, R., Wilkie, D., Byron, E., Huanca, T., Leonard, W., et al. (2003). Markets and the use of wild animals for traditional medicine among Tsimane Amerindians, Bolivia. Journal of Ethnobiology, 23, 47-64.

Aswani, S., Albert, S., Sabetian, A., \& Furusawa, T. (2007). Customary management as precautionary and adaptive principles for protecting coral reefs in Oceania. Coral Reefs, 26(4), 1009-1021.

Atran, S. (1999). Managing the Maya commons: The value of local knowledge. In V. Nazarea (Ed.), Ethnoecology: Situated Knowledge, Located Lives. Arizona: University of Arizona Press.

Atran, S., Medin, D. L., Ross, N., Lynch, E., Vapnarsky, V., Ucan Ek', E., et al. (2002). Folkecology, cultural epidemiology, and the spirit of the commons: A garden experiment in the Maya Lowlands, 1991-2001. Current Anthropology, 43(3).

Atran, S., \& Sperber, D. (1991). Learning without teaching: Its place in culture. In L. T. Landsmann (Ed.), Culture, Schooling, and Psychological Development. Norwood, New Jersey: Ablex Publishing Corporation.

Aunger, R. (2000). The life history of culture learning in a face-to-face society. Ethos, 28(3), 445-481.

Baeraleo, S. (2004). Community knowledge and values: Implementing Vanuatu's curriculum. In K. Sanga, J. Niroa, K. Matai \& L. Crowl (Eds.), Re-thinking Vanuatu Education Together. Port Vila: University of the South Pacific.

Baeraleo, S. (2010). Teaching Indigenous Knowledge and Resource Management in the Primary School. Port Vila: The Vanuatu National Cultural Council.

Bajunid, I. A. (2008). The building of a national and ideas of nationhood: Citizenship education in Malaysia. In D. L. Grossman, W. O. Lee \& K. J. Kennedy (Eds.), Citizenship Curriculum in Asia and the Pacific. Hong Kong: Springerlink.

Barbour, J. (2011). Exploring mood in Neverver. Oceanic Linguistics, 50(1), 198-220.

Barelli, M. (2010). The interplay between global and regional human rights systems in the construction of the indigenous rights regime. Human Rights Quarterly: A Comparative and International Joumal of the Social Sciences, Humanities, and Law, 32(4), 951-979. 
Bates, P. (2009). Learning and Inuit knowledge in Nunavat, Canada. In P. Bates, M. Chiba, S. Kube \& D. Nakashima (Eds.), Learning and Knowing in Indigenous Societies Today. Paris: UNESCO.

Becker, D., \& Ostrom, E. (1995). Human ecology and resource sustainability: The importance of institutional diversity. Annual Review of Ecology and Systematics, 26, 113-133.

Bedford, S., \& Galipaud, J.-C. (2011). Chain of islands: Lapita in the north of Vanuatu. In M. C. Sand \& S. Bedford (Eds.), Lapita: Oceanic Ancestors. Paris: Musee du quai Branly.

Begossi, A. (1996). Use of ecological methods in ethnobiology: Diversity indices. Economic Botany, 50(3), 280-289.

Begossi, A., Hanazaki, N., \& Peroni, N. (2000). Knowledge and use of biodiversity in Brazilian hotspots. Environment, Development and Sustainability, 2, 177-193.

Begossi, A., Hanazaki, N., \& Tamashiro, J. (2002). Medicinal plants in the Atlantic Forest (Brazil): Knowledge, use, and conservation. Human Ecology 30(3), 281-299.

Benz, B., Cevallos, J., Santana, F., Rosales, J., \& Graf, S. (2000). Losing knowledge about plant use in the Sierra de Manantlan Biosphere reserve, Mexico. Economic Botany, 54(2), 1874.

Berkes, F. (2008). Sacred Ecology: Traditional Ecological Knowledge and Resource Management (2 ed.). London: Taylor and Francis.

Berkes, F., Colding, J., \& Folke, C. (2000). Rediscovery of traditional ecological knowledge as adaptive management. Ecological Applications, 10(5), 1251-1262.

Berkes, F., \& Folke, C. (1998). Linking social and ecological systems for resilience and sustainability. In F. Berkes, C. Folke \& J. Colding (Eds.), Linking Social and Ecological Systems: Management Practices and Social Mechanisms for Building Resilience. Cambridge: Cambridge University Press.

Berkes, F., \& Folke, C. (2002). Back to the future: Ecosystem dynamics and local knowledge. In L. Gunderson \& C. S. Holling (Eds.), Panarchy: Understanding Transformations in Human and Natural Systems. Washington: Island Press.

Berkes, F., \& Jolly, D. (2001). Adapting to climate change: Social-ecological resilience in a Canadian Western Arctic community. Conservation Ecology, 5(2), 18.

Berkes, F., \& Turner, N. (2006). Knowledge, learning and the evolution of conservation practice for socio-ecological system resilience. Human Ecology, 34(4).

Berlin, E. (1999). Ecological, sociocultural, and biological determinants of ethnoepidemiological patterns among Tzotzil and Tzeltal Maya. In T. Gragson \& B. Blount (Eds.), Ethnoecology: Knowledge, Resources, and Rights. Athens, Georgia: The University of Georgia Press.

Bernard, H. R. (2005). Research methods in anthropology: Qualitative and quantitative approaches (4 ed.). Walnut Creek, CA: AltaMira Press. 
Bernard, H. R. (Ed.). (1998). Handbook of Methods in Cultural Anthropology. California: AltaMira Press.

Billo, M., Fournet, A., Cabalion, P., Waikedre, J., Bories, C., loiseu, P., et al. (2005). Screening of New Caledonian and Vanuatu medicinal plants for antiprotozoal activity. Journal of Ethnopharmacology, 96, 596-575.

Bishop, R. (2005). Freeing ourselves from neocolonial domination in research: A Kaupapa Maori approach to creating knowledge. In N. Denzin \& Y. Lincoln (Eds.), The SAGE Handbook of Qualitative Research (3 ed). Thousand Oaks: SAGE.

Blakeney, M. (2011). Protecting traditional knowledge and expressions of culture in the Pacific. Queen Mary Journal of Intellectual Property 1(1), 80-89.

Bolton, L. (1999a). Introduction. Oceania, 70(1), 1-8.

Bolton, L. (1999b). Radio and the redefinition of kastom in Vanuatu. The Contemporary Pacific, 11(2), 335360.

Bolton, L. (1999c). Women, place, and practice in Vanuatu: A view from Ambae. Oceania, 70, 43-56.

Bolton, L. (2003). Unfolding the Moon: Enacting Women's Kastom in Vanuatu. Honolulu: University of Hawaii Press.

Bonnemaison, J. (1996). A web of connections. In J. Bonnemaison, K. Huffman, C. Kaufmann \& D. Tryon (Eds.), Arts of V anuatu. Honolulu: University of Hawaii Press.

Bonnemaison, J., Huffman, K., Kaufmann, C., \& Tryon, D. (Eds.). (1996). Arts of V anuatu. Bathurst: Crawford House.

Borgatti, S. (1996). Anthropac 4. Natick MA: Analytic Technologies.

Boster, J. (1986). Exchange of varieties and information between Aguaruna manioc cultivators. American Anthropologist, 88, 429-436.

Botha, L. (2010). Indigenous knowledge as culturally-centred education in South Africa. Africa Education Review, 7(1), 34-50.

Bourdy, G., Francois, C., Andary, C., \& Boucard, M. (1996). Maternity and medicinal plants in Vanuatu II: Pharmacological screening of five selected plants. Joumal of Ethnopharmacology, 52, 139-143.

Bourdy, G., \& Walter, A. (1992). Maternity and medicinal plants in Vanuatu I: The cycle of reproduction. Journal of Ethnopharmacology, 37, 176-196.

Boyd, R., \& Richerson, P. (1985). Culture and the Evolutionary Process. Chicago: University of Chicago Press.

Bradacs, G. (2008). Ethnobotanical survey and biological screening of medicinal plants from Vanuatu. Universitat Regensburg, Frankfurt. 
Bradacs, G., Heilmann, J., \& Weckerle, C. (2011). Medicinal plant use in Vanuatu: A comparative ethnobotanical study of three islands. Journal of Ethnopharmocology, 137(1), 434-448.

Brayboy, B., \& Castagno, A. (2009). Self-determination through self-education: Culturally responsive schooling for indigenous students in the USA. Teaching Education, 20(1), 31-53.

Brechin, S., Wilshusen, P., Fortwangler, C., \& West, P. (2002). Beyond the square wheel: Towards a more comprehensive understanding of biodiversity conservation as a social and political process. Society \& Natural Resources, 15, 41-64.

Bregulla, H. (1992). Birds of V anuatu. Oswestry: Antony Nelson.

Bridges, K., \& McClatchey, W. (2009). Living on the margin: Ethnoecological insights from Marshall Islanders at Rongelap Atoll. Global Environmental Change, 19(2), 140-146.

Briggs, J. (2005). The use of indigenous knowledge in development: Problems and challenges. Progress in Development Studies, 5(2), 99-114.

Briggs, J., \& Sharp, J. (2004). Indigenous knowledges and development: A postcolonial caution. Third World Quarterly, 25(4), 661-676.

Brodt, S. (2001). A Systems Perspective on the Conservation and Erosion of Indigenous Agricultural Knowledge in Central India. Human Ecology, 29(1), 99-120.

Brokensha, D., Warren, B., \& Werner, O. (1980). Indigenous Knowledge Systems and Development. Washington, DC: University Press of America.

Brosi, B., Balick, M., Wolkow, R., Lee, R., Kostka, M., Raynor, W., et al. (2007). Cultural erosion and biodiversity: Canoe-making knowledge in Pohnpei, Micronesia Conservation Biology, 21(3), 875-879.

Brosius, J. P. (1997). Endangered forests, endangered people: Environmentalist representations of indigenous knowledge. Human Ecology, 25(1), 47-69.

Burnett, G. (2007). 'Culture isn't what it used to be': Problematising pedagogies of cultural difference in Pacific education. Social and Economic Studies, 56(1/2), 261.

Burnett, G. (2009). Critically theorising the teaching of literacy and language in Pacific schooling: Just another Western metanarrative? Critical Literacy: Theories and Practices, 3(2).

Burnham, K., \& Anderson, D. (1998). Model Selection and Inference: A Practical Information-Theoretic Approach. New York: Springer.

Burnham, K., \& Anderson, D. (2004). Multimodel Inference: Understanding AIC and BIC in Model Selection. Sociological Methods and Research, 33, 261-304.

Byers, B., Cunliffe, R., \& Hudak, A. (2001). Linking the conservation of culture and nature: A case study of sacred forests in Zimbabwe. Human Ecology, 29(2), 187. 
Byg, A., \& Balslev, H. (2001). Traditional knowledge of Dypsis fibrosa (Arecaceae) on Eastern Madagascar. Economic Botany, 55(2), 263.

Byg, A., \& Balslev, H. (2004). Factors affecting local knowledge of palms in Nangaritza Valley, southeastern Ecuador. Journal of Ethnobiology 24(2), 255-278.

Cabalion, P., Sam, C., \& Hoff, M. (1991). Contribution a l'Etude de la Flore de Vanuatu. Index Synonymique de la Flore, Liste de Specimens d'Herbier par Especes, Listes des Especes par Ile [Document Interne]. Port Vila, Vanuatu: OSRTOM.

Caillon, S., Quero-Garcia, J., \& Guarino, L. (2004). Taro in Vanuatu: Towards a dynamic conservation strategy. Leisa Magazine, March.

Campbell, J. (1990). Disasters and development in historical context: Tropical cyclone response in the Banks Islands, Northern Vanuatu. International Journal of Mass Emergencies and Disasters, 8(3), 404424.

Campbell, J. (2006). Traditional disaster reduction in Pacific Island communities. GNS Science Report (2006/038).

Caniago, I., \& Siebert, S. (1998). Medicinal plant economy, knowledge, and conservation in Kalimantan, Indonesia. Economic Botany, 52(3), 229-250.

Carpenter, S., Walker, B., Anderies, J. M., \& Abel, N. (2001). From metaphor to measurement: Resilience of what to what? Ecosystems, 4, 765-781.

Casagrande, D. G. (2002). Ecology, Cognition, and Cultural Transmission of Tzeltal Maya Medicinal Plant Knowledge (PhD dissertation). University of Georgia, Athens, Georgia.

Case, R., Pauli, G., \& Soejarto, D. (2005). Factors in maintaining indigenous knowledge among ethnic communities of Manus Island. Economic Botany, 59(4), 356-365.

Castagno, A., \& Brayboy, B. (2008). Culturally responsive schooling for indigenous youth: A review of the literature. Revien of Education Research, 78(4), 941-993.

Cavalli-Sforza, L., \& Feldman, M. W. (1981). Cultural Transmission and Evolution: A Quantitative Approach. Princeton, NJ: Princeton University Press.

Cavalli-Sforza, L. L., Feldman, M. W., Chen, K. H., \& Dornbusch, S. M. (1982). Theory and observation in cultural transmission. Science, 218(4567), 19-27.

Chambers, M. (1992). Introduction. In H. Bregulla (Ed.), Birds of V anuatu. Owestry: Anthony Nelson.

Chang Bioscience. (2011). Shannon-Wiener Diversity Index / Shannon Entropy Calculator. Retrieved 20th April, 2011, from http://www.changbioscience.com/genetics/shannon.html 
Charnley, S., Paige Fischer, A., \& Jones, E. (2007). Integrating traditional and local ecological knowledge into forest biodiversity conservation in the Pacific Northwest Forest Ecology and Management, 246, $14-28$.

Charpentier, J.-M. (1982). Atlas Lingiustique du Sud-Malakula (V anuatu). Paris: Societe D'etudes Linguistiques et Anthropologiques de France.

Chipenuik, R. (1995). Childhood foraging as a means of acquiring competent human cognition about biodiversity. Environment and Behavior, 24(4), 490-514.

Clapp, J., \& Dauvergne, P. (2005). Paths to a Green World: The Political Economy of the Global Environment. Cambridge, Massachusetts: The MIT Press.

Cocks, M. (2006). Biocultural diversity: Moving beyond the realm of 'indigenous' and 'local' people. Human Ecology, 34(2), 185-202.

Coe, F., \& Anderson, D. (1996). Ethnobotany of the Garifuna of Eastern Nicaragua. Economic Botany, 50, 71-107.

Colwell, R. K. (2009). EstimateS: Statistical estimation of species richness and shared species samples. Version 8.2. User's Guide and application published at: http://purl.oclc.org/estimates.

Colwell, R. K., Mao, C. X., \& Chang, J. (2004). Interpolating, extrapolating, and comparing incidencebased species accumulation curves. Ecology 85(10), 2717-2727.

Conservation International. (2009). East Melanesian Islands Hotspot. Retrieved 18/10, 2011, from http://www.biodiversityhotspots.org/xp/hotspots/east_melanesia/Pages/default.aspx

Cox, P. (1999). Nafanua: Saving the Samoan Rainforest. New York: W.H Freeman.

Cox, P. (2000). Will tribal knowledge survive the millennium? Science, 287(5450), 44-45.

Crawford, S. (2009). Matauranga Maori and western science: The importance of hypotheses, predictions, and protocols. Journal of the Royal Society of New Zealand, 39(4), 163-166.

Creswell, J., \& Plano Clark, V. (2007). Designing and conducting mixed methods research. London: SAGE Publications.

Cristancho, S., \& Vining, J. (2009). Perceived intergenerational differences in the transmission of traditional ecological knowledge (TEK) in two indigenous groups from Columbia and Guatemala. Culture and Psychology, 15, 229.

Cronin, S., Gaylord, R., Charley, D., Alloway, B., Wallex, S., \& Esau, J. (2004). Participatory methods of incorporating scientific with traditional knowledge for volcanic hazard management on Ambae Island, Vanuatu. Bulletin of Volcanology, 66, 652-668.

Crowley, T. (1990). From Beach-la-Mar to Bislama: The emergence of a national language in Vanuatu. Oxford and New York: Clarendon Press. 
Crowley, T. (2000). Vernaculars in Education in Vanuatu. Port Vila: World Bank \& Vanuatu Ministry of Education, Youth and Sport.

Crowley, T. (2003). A New Bislama Dictionary (2 ed). Port Vila: University of the South Pacific, Pacific Languages Unit.

Crowley, T. (2006a). The Avava Language of Central Malakula (V anuatu). Canberra: Pacific Linguistics, Australian National University.

Crowley, T. (2006b). Naman: A V anishing Language of Malakula (Vanuatu). Canberra: Pacific Institute of Linguistics, Australian National University.

Crowley, T. (2006c). Nese: A Diminishing Speech Variety of Nortbwest Malakula (Vanuatu). Canberra: Pacific Institute of Linguistics, Australian National University.

Crowley, T. (2006d). Tape: A Declining Language of Malakula. Canberra: Pacific Institute of Linguistics, Australian National University.

Cruz Garcia, G. S. (2006). The mother-child nexus: Knowledge and valuation of wild food plants in Wayanad, Western Ghats, India. Journal of Ethnobiology and Ethnomedicine, 2(39).

Curtis, T. (1999). Tom's Tambu House: Spacing, status, and sacredness in South Malakula, Vanuatu. Oceania, 70, 56-71.

Curtis, T. (2002). Talking about place: Identities, histories, and powers amongst the Na'hai speakers of Malakula (Vanuatu). Australian National University, Canberra.

Davis, A., \& Wagner, J. (2003). Who knows? On the importance of identifying 'experts' when researching local ecological knowledge. Human Ecology 31(3), 463-489.

Davis, W. (1996). One River: Explorations and Discoveries in the Amazon Rain Forest. New York: Simon and Schuster.

Davis, W. (2009). Light at the Edge of the World: A Journey Through the Realm of Vanishing Cultures. Vancouver: Douglas and McIntyre.

Davis, W., \& Henley, T. (Eds.). (1990). Penan: Voice for the Borneo Rainforest. Vancouver: Western Canada Wilderness Committee.

De Beer, J., \& Whitlock, E. (2009). Indigenous Knowledge in the Life Sciences Classroom: Put on Your de Bono Hats! The American Biology Teacher, 71(4), 209-216.

De Sousa Santos, B. (2007). Another Knowledge Is Possible: Beyond Northern Epistemologies. London: Verso.

Deacon, B. (1934). Malekula: A Vanishing People in the New Hebrides. London: George Routledge \& Sons, Ltd. 
Delors, J. (1996). Learning: The Treasure Within. Paris: UNESCO.

Delors, J. (2002). Learning Throughout Life: Challenges for the Twenty-First Century. Paris: UNESCO.

Dewey, J. (1937). Education and social change. Bulletin of the American Association of University Professors, 23(6), 472-474.

Diamond, J. (2005). Collapse: How Societies Choose to Fail or Succeed. US: Viking Press.

Diamond, J., \& Bishop, K. (1999). Ethno-ornithology of the Ketengban people, Indonesian New Guinea.

In D. L. Medin \& S. Atran (Eds.), Folkbiology. Cambridge: The MIT Press.

Dietz, T., Ostrom, E., \& Stern, P. (2003). The struggle to govern the commons. Science, 302(1907).

Don, W. (2010). The titi project, traditional ecological knowledge and science: A critique. Journal of the Royal Society of New Zealand, 40(2), 39-43.

Dove, M. (2000). The life-cycle of indigenous knowledge, and the case of natural rubber production. In R. Ellen, P. Parkes \& A. Bicker (Eds.), Indigenous Environmental Knowledge and its Transformations: Critical Anthropological Perspectives. London and New York: Routledge.

Dove, M. (2002). Hybrid histories and indigenous knowledge among Asian rubber smallholders.

International Social Science Journal, 173, 349-359.

Dove, M., Smith, D., Campos, M., Mathews, A., Rademacher, A., Rhee, S., et al. (2007). Globalisation and the construction of western and non-western knowledge. In P. Sillitoe (Ed.), Local vs. Global Knowledge: Approaches to Indigenous Knowledge in Global Development. New York: Berghahn books.

Drew, J. (2005). Use of traditional ecological knowledge in marine conservation. Conservation Biology, 19(4), 1286-1293.

Ellen, R., \& Harris, H. (2000). Introduction. In R. Ellen, P. Parkes \& A. Bicker (Eds.), Indigenous Environmental Knowledge and its Transformations. London and New York: Routledge.

Ellen, R., Parkes, P., \& Bicker, A. (Eds.). (2000). Indigenous Environmental Knowledge and its Transformations: Critical Anthropological Perspectives. Amsterdam: Harwood Academic Publishers Ltd.

England, K. (1994). Getting personal: Reflexivity, positionality, and feminist research. Professional Geographer, 46(1), 80-89.

Escobar, A. (1995). Encountering development: The making and unmaking of the Third World. Princeton NJ: Princeton University Press.

Estomba, D., Ladio, A., \& Lozada, M. (2006). Medicinal wild plant knowledge and gathering patterns in a Mapuche community from north-western Patagonia. Journal of Ethnopharmacology, 103, 109-119.

Eyssartier, C., Ladio, A., \& Lozada, M. (2008). Cultural transmission of traditional knowledge in two populations of North-West Patagonia. Journal of Ethnobiology and Ethnomedicine, 4(25). 
Field, A. (2009). Discovering Statistics Using SPSS: Third Edition. Thousand Oaks, CA: SAGE.

Fishman, J. (2001). Can threatened languages be saved? Reversing language shift, revisited: A 21st century perspective. Cleveland: Multilingual Matters Ltd.

Flannery, T. (2002). The Future Eaters: An Ecological History of the Australasian Lands and People. New York: Grove Press.

Florey, M. (2009). Sustaining indigenous languages and indigenous knowledge: Developing community training approaches for the 21st century. In P. Bates, M. Chiba, S. Kube \& D. Nakashima (Eds.), Learning and Knowing in Indigenous Societies Today. Paris: UNESCO.

Foale, S. (2006a). The intersection of scientific and indigenous knowledge in coastal Melanesia: Implications for contemporary marine resource management. International Social Science Journal, 58(187), 129-137.

Foale, S. (2006b). Is coral reef conservation possible without science education in Melanesia? Is science education possible without development? Proceedings of the 10th International Coral Reef Symposium

Folke, C., Berkes, F., \& Colding, J. (1998). Ecological practices and social mechanisms for building resilience and sustainability. In F. Berkes, C. Folke \& J. Colding (Eds.), Linking Social and Ecological Systems: Management Practices and Social Mechanisms for Building Resilience. Cambridge: Cambridge University Press.

Folke, C., Carpenter, S., Elmqvist, T., Gunderson, L., Holling, C. S., Walker, B., et al. (2002). Resilience and Sustainable Development: Building Adaptive Capacity in a World of Transformation: Environmental Advisory Council to the Swedish Government.

Folke, C., Colding, J., Olsson, P., \& Hahn, T. (2007). Interdependent social-ecological systems and adaptive governance for ecosystem services. In J. Pretty, A. Ball, T. Benton, J. Guivant, D. Lee, D. Orr, M. Pfeffer \& H. Ward (Eds.), The SAGE Handbook of Environment and Society. London: SAGE Publications Ltd.

Ford, J., \& Martinez, D. (2000). Traditional ecological knowledge, ecosystem science, and environmental management. Ecological Applications, 10(5), 1249-1250.

Funabiki, T. (1981). On pigs of the Mbotkot in Malekula. In M. Allen (Ed.), Vanuatu: Politics, Economics, and Ritual in Island Melanesia. Sydney.

Furusawa, T. (2006). Role of Western biomedicine and folk medicine in rural Solomon Islands: a quantitative analysis of villagers response to illness. Tropical Medicine and Health, 34(2), 83-91.

Furusawa, T. (2009). Changing ethnobotanical knowledge of the Roviana people, Solomon Islands: Quantitative approaches to its correlation with modernity. Human Ecology, 37, 147-159. 
Gadgil, M., Hemam, N. S., \& Reddy, B. H. (1998). People, refugia and resilience. In F. Berkes, C. Folke \& J. Colding (Eds.), Linking Social and Ecological Systems: Management Practices and Social Mechanisms for Building Resilience. Cambridge: Cambridge University Press.

Gadgil, M., Rao, P. R. S., Utkarsh, G., \& Chhartre, A. (2000). New meanings for old knowledge: The people's biodiversity registers programme. Ecological Applications, 10(5), 1307-1317.

Garanger, J. (1996). Preface. In J. Bonnemaison, K. Huffman, C. Kaufmann \& D. Tryon (Eds.), Arts of Vanuatu. Honolulu: University of Hawaii Press.

Garro, L. C. (1986). Intracultural Variation in Folk Medical Knowledge: A Comparison between Curers and Noncurers. American Anthropologist, 88(2), 351-370.

Gavin, M. C., \& Anderson, G. J. (2007). Socioeconomic predictors of forest use values in the Peruvian Amazon: A potential tool for biodiversity conservation. Ecological Economics, 60, 752-762.

Geismar, H. (2009). Stone men of Malekula on Malekula: An ethnography of an ethnography. Ethnos, 74(2), 199-228.

Ghimire, S. K., McKey, D., \& Aumeeruddy-Thomas, Y. (2004). Heterogeneity in ethnoecological knowledge and management of medicinal plants in the Himalayas of Nepal: Implications for conservation. Ecology and Society, 9(3), 6.

Godoy, R., Brokaw, N., Wilkie, D., Colon, D., Palermo, A., Lye, S., et al. (1998). Of trade and cognition: Markets and the loss of folk knowledge among the Tawahka Indians of the Honduran rainforest. Journal of Anthropological Research, 54(2), 219-234.

Godoy, R., Reyes-Garcia, V., Broesch, J., Fitzpatrick, I., Giovanninni, P., Rodriguez, M., et al. (2009). Long-term (secular) change of ethnobotanical knowledge of useful plants: Separating cohort and age effects. Journal of Anthropological Research, 65, 51-67.

Godoy, R., Reyes-Garcia, V., Byron, E., Leonard, W., \& Vadez, V. (2005). The effect of market economies on the well-being of indigenous peoples and on their use of renewable natural resources. Annual Review of Anthropology, 34, 121-138.

Gomez-Baggethun, E., Mingorria, S., Reyes-Garcia, V., Calvet, L., \& Montes, C. (2010). Traditional ecological knowledge trends in the transition to a market economy: empirical study in the Donanan Natural Areas. Conservation Biology, 24(3), 721-729.

Gorecki, P. (1996). The initial colonisation of Vanuatu. In J. Bonnemaison, K. Huffman, C. Kaufmann \& D. Tryon (Eds.), Arts of V anuatu. Honolulu: University of Hawaii Press.

Gotelli, N. J., \& Colwell, R. K. (2001). Quantifying biodiversity: Procedures and pitfalls in the measurement and comparison of species richness. Ecology Letters, 4, 379-391. 
Gotelli, N. J., \& Colwell, R. K. (2011). Estimating species richness. In A. E. Magurran \& B. J. McGill (Eds.), Biological Diversity: Frontiers in Measurement and Assessment. Oxford: Oxford University Press.

Grace, R. (2001). Anticholinergic poisoning secondary to custom medicine in Vanuatu. Journal of Ethnopharmacology, 77, 269-270.

Gray, R., Drummond, A., \& Greenhill, S. (2009). Language phylogenies reveal expansion pulses and pauses in Pacific settlement. Science 323, 479.

Greenfield, P., \& Lave, J. (1982). Cognitive aspects of informal education. In D. Wagner \& H. Stevenson (Eds.), Cultural Perspectives on Child Development. San Francisco, CA: Freeman.

Greenfield, P. M., Maynard, A. E., \& Childs, C. P. (2000). History, culture, learning, and development. Cross-Cultural Research, 34(4), 351-374.

Gregory, R. J., \& Gregory, J. E. (2000). The custom school and gender equality. Anthropologist, 2(3), 197198.

Guba, E., \& Lincoln, Y. (2005). Paradigmatic controversies, contradictions, and emerging confluences. In N. Denzin \& Y. Lincoln (Eds.), The SAGE Handbook of Qualitative Research (3 ed). Thousand Oaks: SAGE.

Guest, G. (2002). Market integration and the distribution of ecological knowledge within an Ecuadorian fishing community. Journal of Ecological Anthropology, 6(1), 38.

Haccius, J. (2011). The Interaction of Modern and Custom Land Tenure Systems in Vanuatu. Canberra: ANU School of International, Political, and Strategic Studies.

Hae Rokona. (2011). Reef and Rainforest: An Environmental Wiki of Marovo Lagoon, Solomon Islands. Retrieved 11th October, 2011, from http://ma.marovo.org/index.php?title=Hae_Rokona Hanazaki, N., Tamashiro, J. Y., Leitão-Filho, H. F., \& Begossi, A. (2000). Diversity of plant uses in two Caiçara communities from the Atlantic Forest coast, Brazil. Biodiversity and Conservation, 9(5), 597615.

Harmon, D. (2002). In Light of our Differences: How Diversity and Culture Makes Us Human. Washington and London: Smithsonian Institution Press.

Harrison, D. K. (2007). When Languages Die: The Extinction of the World's Languages and the Erosion of Human Knowledge. Oxford: Oxford University Press.

Harrison, T. (1936). Living with the people of Malekula. The Geographic Journal, 88(2), 97-124.

Haugen, E. (1972). The Ecology of Languages: Essays by Einar Haugen. Sanford: Stanford University Press.

Heckler, S. (2002). Traditional ethnobotanical knowledge loss and gender among the Piaroa. In J. R. Stepp, F. S. Wyndham \& R. K. Zarger (Eds.), Ethnobiology and Biocultural Diversity. Athens, Georgia: The International Society of Ethnobiology. 
Heckler, S. (2007). On knowing and not knowing: The many valuations of Piaroa local knowledge. In P. Sillitoe (Ed.), Local Science vs. Global Science: Approaches to Indigenous Knowledge in International Development. New York: Berghahn Books.

Herdt, G. (1984). Ritualized homosexual behavior in the male cults of Melanesia, 1862-1983: An introduction. In G. Herdt (Ed.), Ritualized Homosexuality in Melanesia. London: University of California Press.

Hewlett, B. S., \& Cavalli-Sforza, L. (1986). Cultural transmission among Aka Pygmies. American Anthropologist, 88(922-934).

Hewlett, Barry S., De Silvestri, A., \& Guglielmino, C. R. (2002). Semes and Genes in Africa. Current Anthropology, 43(2), 313-321.

Hickey, F. (2003a). Traditional Marine Resource Management in Vanuatu: Worldviews in Transformation; Sacred and Profane. Paper presented at the Putting Fishers' Knowledge to Work Conference, University of British Columbia.

Hickey, F. (2003b). The Use of Indigenous Knowledge in Traditional Fisheries and Marine Resource Management in Vanuatu. Port Vila: Vanuatu Cultural Centre.

Hickey, F. (2005). Recognising, Acknowledging, Strengthening and Supporting Traditional Resource Management Practices. Paper presented at the National Summit on Self-Reliance and Sustainability: Port Vila.

Hickey, F. (2006a). Amal/Crab Bay Tabu Eria Review, Malekula Island. Apia, Samoa: SPREP.

Hickey, F. (2006b). Traditional marine resource management in Vanuatu: Acknowledging, supporting and strengthening indigenous management systems. SPC Traditional Marine Resource Management and Knowledge Information Bulletin 20, 11-24.

Hickey, F., \& Ruddle, K. (2008). Accounting for the mismanagement of tropical nearshore fisheries. Environmental Development and Sustainability, 10(5), 565-589.

Huffman, K. (1996). Trading, cultural exchange, and copyright: Important aspects of Vanuatu arts. In J. Bonnemaison, K. Huffman, C. Kaufmann \& D. Tryon (Eds.), Arts of Vanuatu. Honolulu: University of Hawaii Press.

Hunn, E. S. (1999). The value of subsistence for the future of the world. In V. Nazarea (Ed.), Ethnoecology: Situated Knowledge, Located Lives. Arizona: University of Arizona Press.

Hunn, E. S. (2002). Evidence for the precocious acquisition of plant knowledge by Zapotec children In J. R. Stepp, F. S. Wyndham \& R. K. Zarger (Eds.), Ethnobiology and Biocultural Diversity. Athens, GA: International Society for Ethnobiology.

Huntingdon, H. (2000). Using traditional ecological knowledge as science: Methods and applications. Ecological Applications, 10(5), 1270-1274. 
Hviding, E. (2005). Village-level document and transmission of local environmental knowledge: A pilot for the Solomon Islands. Retrieved from http://portal.unesco.org/science/en/ev.phpURL_ID=4989\&URL_DO=DO_TOPIC\&URL_SECTION=201.html

Inglehart, R., \& Baker, W. (2000). Cultural change and the persistence of traditional values. American Sociological Review, 65(1), 19-51.

International Council for Science. (2002). Science, Traditional Knowledge and Sustainable Development. Paris: UNESCO.

International Society of Ethnobiology. (1988). Declaration of Belem. Retrieved 21st October, 2011, from http://ethnobiology.net/docs/DeclarationofBelem.pdf

International Society of Ethnobiology. (2006). ISE Code of Ethics (with 2008 additions). Retrieved 19 June, 2009, from http://ethnobiology.net/code-of-ethics/code-in-english/

Jackson, J., Kirby, M., Berger, W., Bjorndal, K., Botsford, L., Bourque, B., et al. (2001). Historical overfishing and the recent collapse of coastal ecosystems. Science, 293, 623-637.

Johannes, R. (1981). Words of the Lagoon: Fishing and Marine Lore in the Palau District of Micronesia. Berkeley: University of California Press.

Johannes, R. (1998). The case for data-less marine resource management: Examples from tropical nearshore finfisheries. Trends in Ecology \& Evolution, 13(6), 243-246.

Johannes, R. (2002). Did indigenous conservation ethics exist? Traditional Marine Resource Management and Knowledge Information Bulletin, 14, 3-6.

Jolly, M. (1994). Women of the Place: Kastom, Colonialism and Gender in Vanuatu. Camberwell, Victoria: Harwood Academic Publishers.

Kates, R., Clark, W. C., Corell, R., Hal, J., Jaeger, C., Lowe, I., et al. (2001). Sustainability science. Science, 292, 641 .

Keesing, R. (1985). Kwaio women speak: The micropolitics of autobiography in a Solomon Island society. American Anthropologist, 87(1), 27-40.

Kellner, K., \& Bosch, O. (2003). The value of local and indigenous knowledge for the development of information systems for conservation management. In J. Lemons, R. Victor \& D. Schaffer (Eds.), Conserving Biodiversity in Arid Regions. USA: Kluwer Academic Publishers.

Kimmerer, R. (2002). Weaving traditional ecological knowledge into biological education: A call to action. Bioscience, 52 (5), 432-439.

King, S. (1996). Conservation and tropical medicinal plant research. In M. Balick, E. Elisabetsky \& S. Laird (Eds.), Medicinal Resources of the Tropical Forest. New York: Columbia University Press. 
Kitchin, R., \& Tate, N. (2000). Conducting Research in Human Geography: Theory, Methodology, and Practice. London: Prentice Hall.

Korean Traditional Knowledge Portal. (2011). Korean Traditional Knowledge Portal. Retrieved 11th October, 2011, from http://www.koreantk.com/en/JZ0100.jsp

Krauss, M. (1992). The world's languages in crisis. Language, 68(1), 4-10.

Krauss, M. (2007). Mass language extinction and documentation: The race against time. In O. Miyaoka, O. Sakiyama \& M. Krauss (Eds.), The Vanishing Languages of the Pacific Rim. Oxford: Oxford University Press.

Kristensen, M., \& Balslev, H. (2003). Perceptions, use and availability of woody plants among the Gourounsi in Burkina Faso. Biodiversity and Conservation, 12(8), 1715-1739.

Kumar, S. (2002). Methods for Community Participation: A Complete Guide for Practitioners. Warwickshire, UK: Intermediate Technology Publications Ltd.

La Belle, T. (1982). Nonformal and informal education: A holistic perspective on lifelong learning. International Review of Education, 28(2), 159-175.

Ladio, A., \& Lozada, M. (2004). Patterns of use and knowledge of wild edible plants in distinct ecological environments: A case study of a Mapuche community from north-western Patagonia. Biodiversity and Conservation, 13, 1153-1173.

Lake, B. (1985). The Educational Policy of the Presbyterian Church of the New Hebrides (Vanuatu) 1948-1980. Murdoch University, Perth.

Lancy, D. (1996). Playing on the Mother Ground: Cultural Routines for Children's Development. New York, London: The Guildford Press.

Lane, M. (2006). Evaluating the Governance of Coastal Resources and Environments in Vanuatu. Apia: South Pacific Regional Environment Program (SPREP):

Larcom, J. (1990). Custom by decree: Legitimation crisis in Vanuatu. In J. Linnekin \& L. Poyer (Eds.), Cultural Identity and Ethnicity in the Pacific. Honolulu: University of Hawaii Press.

Lave, J., \& Wenger, E. (1991). Situated Learning: Legitimate Peripheral Participation. Cambridge: Cambridge University Press.

Lavoue, J., \& Droz, P. (2009). Multimodel Inference and Multimodel Averaging in Empirical Modelling of Occupational Exposure Levels. The Annals of Occupational Hygiene, 53(2), 1-8.

Layard, J. (1942). Stone Men of Malekula. London: Chatto \& Windus.

Lee, R. A., Balick, M., Ling, D., Sohl, F., Brosi, B., \& Raynor, W. (2001). Cultural dynamism and change: An example from the Federated States of Micronesia. Economic Botany, 55(1), 9-13. 
Lertzman, D. (2002). Rediscovering rites of passage: Education, transformation, and the transition to sustainability. Conservation Ecology, 5(2), 30.

LeVine, R. (1984). Properties of culture: An ethnographic view. In R. Shweder \& R. LeVine (Eds.), Culture Theory: Essays on Mind, Self, and Society. Cambridge, MA: Harvard University Press.

Lewis, M. (2009). Ethnologue: Languages of the World, Sixteenth Edition. Dallas, Texas: SIL International.

Lewis, W. H. (2003). Pharmaceutical discoveries based on ethnomedicinal plants: 1985 to 2000 and beyond. Economic Botany, 57(1), 126-134.

Lieske, E., \& Myers, R. (1999). Coral Reef Fishes: Caribbean, Indian Ocean, and Pacific Ocean Princeton: Princeton University Press.

Lightner, S., \& Naupa, A. (2005). Histri Blong Yumi Long Vanuatu. Port Vila: Vanuatu National Cultural Council.

Lindstrom, L. (2009). Kava pirates in Vanuatu? International Journal of Cultural Property 16, 291-308

Lipka, J. (1994). Culturally negotiated schooling: Toward a Yup'ik mathematics. Journal of American Indian Education, 33(3).

Lizarralde, M. (2001). Biodiversity and loss of indigenous languages and knowledge in South America. In L. Maffi (Ed.), On Biocultural Diversity: Linking Language, Knowledge, and the Environment. Washington DC: Smithsonian Press.

Louette, D., Charrier, A., \& Berthaud, J. (1997). In situ conservation of Maize in Mexico: Genetic diversity and Maize seed management in a traditional community. Economic Botany, 51(1), 20-38.

Lozada, M., Ladio, A., \& Weigandt, M. (2006). Cultural transmission of ethnobotanical knowledge in a rural community of north-western Patagonia, Argentina. Economic Botany, 60(4), 374-385.

Luke, A. (2009). On indigenous education. Teaching Education, 20(1), 1-5.

Lykke, A. M., Kristensen, M. K., \& Ganaba, S. (2004). Valuation of local use and dynamics of 56 woody species in the Sahel. Biodiversity and Conservation, 13(10), 1961-1990.

Lynch, J. (2005). The apicolabial shift in Nese. Oceanic Linguistics, 44(2), 389-403.

Lynch, J., \& Crowley, T. (2001). Languages of V anuatu. Canberra: Pacific Linguists.

MacClancy, J. (2002). To Kill a Bird With Two Stones. Port Vila: Vanuatu Cultural Centre.

Maclean, R. (2002). Secondary education reform and educational research in the Asia-Pacific region. Educational Research for Policy and Practice, 1, 79-98.

Maffi, L. (2004). Maintaining and restoring biocultural diversity: The evolution of a role for Ethnobiology. In J. S. Carlson \& L. Maffi (Eds.), Ethnobotany and the Conservation of Biocultural Diversity. New York: The New York Botanical Garden Press. 
Maffi, L. (2005). Linguistic, cultural and biological diversity. Annual Review of Anthropology, 29, 599-617.

Maffi, L., \& Woodley, E. (2010). Biocultural Diversity Conservation: A Global Sourcebook. London: Earthscan.

Magurran, A. E. (1988). Ecological diversity and its measurement. Princeton, NJ: Princeton University Press.

Magurran, A. E. (2004). Measuring Biological Diversity. Malden, Ma \& Oxford, UK: Blackwell Science Ltd.

Marahere, D. (2004). Towards an equitable future in Vanuatu: The legal protection of cultural property. Journal of South Pacific Law 8(2).

Marie, D., \& Haig, B. (2009). The Maori renaissance and the politicisation of science in New Zealand. In R. Openshaw \& E. Rata (Eds.), The Politics of Conformity in New Zealand. Auckland, New Zealand: Pearson.

Martin, J., Roy, E., Diemont, S., \& Ferguson, B. (2010). Traditional ecological knowledge (TEK): Ideas, inspiration, and designs for ecological engineering. Ecological Engineering, 36(7), 839-849.

Martinez-Rodriguez, M. (2001). Ethnobotanical Knowledge Acquisition Among Tsimane' Children in the Bolivian Amazon (PhD dissertation). Athens, Georgia: University of Georgia.

Mascia, M., Brosius, J. P., Dobson, T., Forbes, B., Horowitz, L., McKean, M., et al. (2003). Editorial: Conservation and the social sciences. Conservation Biology, 17(3), 649-650.

Matthews, M. (1994). Science Teaching: The Role of History and Philosophy of Science. New York: Routledge Press.

Maynard, A. E. (2002). Cultural teaching: The development of teaching skills in Maya sibling interactions. Child Development, 73(3), 969-982.

Maynard, A. E. (2004). Cultures of teaching in childhood: Formal schooling and Maya sibling teaching at home. Cognitive Development, 19, 517-535.

McCarter, J., \& Gavin, M. C. (2011). Perceptions of the value of traditional ecological knowledge to formal school curricula: Opportunities and challenges from Malekula Island, Vanuatu. Journal of Ethnobiology and Ethnomedicine, 38(7).

McGrath, B. B. (1999). Swimming from island to island: Healing practice in Tonga. Medical Anthropology Quarterly, 13(4).

McGregor, S., Lawson, V., Christopherson, P., Kennett, R., Boyden, R., Bayliss, P., et al. (2010). Indigenous wetland burning: Conserving natural and cultural resources in Australia's World Heritage-listed Kakadu National Park. Human Ecology, 38(721-729).

Medley, K. E., \& Kalibo, H. (2004). An ecological framework for participatory ethnobotanical research at Mt. Kasigau, Kenya. Field Methods, 17(3). 
Mees, C. C., \& Anderson, J. (1999). The performance of customary marine tenure in the management of community fishery resources in Melanesia: Final technical report. London: MRAG.

Mercer, J., Dominey-Howes, D., Kelman, I., \& Lloyd, K. (2007). The potential for combining indigenous and western knowledge in reducing vulnerability to environmental hazards in small island developing states. Environmental Hazards, 7, 245-256.

Mercer, J., Kelman, I., Taranis, L., \& Suchet-Pearson, S. (2010). Framework for integrating indigenous and scientific knowledge for disaster risk reduction. Disaster, 34(1), 214-239.

Metcalf, P. (2002). They Lie, We Lie: Getting On with Anthropology. London and New York: Routledge.

Miles, M., \& Huberman, A. (1994). Qualitative Data Analysis. Thousand Oaks, CA: Sage.

Molisa, G. (1980). Women. In B. Weightman, H. Lini \& W. Lini (Eds.), Vanuatu: Twenti Wan Tingting long Taem blong Independens. Suva: Institute of Pacific Studies, University of the South Pacific.

Moller, H. (2009). Matauranga Maori, science and seabirds in New Zealand. New Zealand Journal of Zoology, 36(2), 203-210.

Moller, H., Kitson, J., \& Downs, T. (2009). Knowing by doing: Learning for sustainable muttonbird harvesting. New Zealand Journal of Zoology, 36(2), 243-258.

Moller, H., Newman, J., Lyver, P., \& Rakiura Titi Islands Administering Body (RTIAB). (2010). Fourteen Years On: Lessons for Community-led Science Partnerships from the Kia Mau ti Titi Mo Ake Tonu Atu project. Paper presented at the Nga Kete a Rehua Inaugural Maori Research Symposium Te Waipounamu 2008, Aotahi, New Zealand.

Moller, H., O'Blyver, P., Bragg, C., Newman, J., Clucas, R., Fletcher, D., et al. (2009). Guidelines for cross-cultural Participatory Action Research Partnerships: A case study of customary seabird harvest in New Zealand. New Zealand Journal of Zoology, 36(2), 211-241.

Morgan, D. (2007). Paradigms lost and pragmatism regained: Methodological implications of combining qualitative and quantitative methods. Journal of Mixed Methods Research, 1(1), 48-76.

Morrow, P., \& Hensel, C. (1992). Hidden dissensions: Minority-majority relationships and the use of contested terminology. Arctic Anthropology, 29(1), 38-53.

Mueller-Dombois, D., \& Fosberg, F. R. (1998). Vegetation of the Tropical Pacific Islands. New York: Springer.

Muhlhausler, P. (1995). The interdependence of linguistic and biological diversity. In D. Myers (Ed.), The Politics of Multiculturalism in the Asia/Pacific. Darwin, Australia: Northern Territory University Press.

Muhlhausler, P. (1996). Linguistic Ecology: Language Change and Linguistic Imperialism in the Pacific Region. London and New York: Routledge.

Nabhan, G. (1995). The dangers of reductionism in biodiversity conservation. Conservation Biology, 9(3), 479-481. 
Nabhan, G. (1998). Passing on a sense of place and traditional ecological knowledge between generations: A primer for Native American museum educators and community-based cultural education projects. People and Plants Handbook, 30-33.

Nadasdy, P. (1999). The politics of TEK: Power and the 'integration' of knowledge. Arctic Anthropology, $36(1 / 2), 1-18$.

Nadasdy, P. (2005). Transcending the debate of the ecologically noble Indian: Indigenous peoples and environmentalism. Ethnohistory, 52(2), 291-331.

Nako, A. (2004). Vernacular language policy in Vanuatu. In K. Sanga, J. Niroa, K. Matai \& L. Crowl (Eds.), Re-thinking V anuatu Education Together. Port Vila: University of the South Pacific.

Nature. (1999). Caution: Traditional knowledge (editorial). Nature, 401(6754), 623.

Navarro, M., Malres, S., Labouisse, J., \& Roupsard, O. (2004). Vanuatu Breadfruit: Survey on botanical diversity and traditional uses of Artocarpus altilus. ISHS Acta Horticulturae, 757.

Nazarea, V. (1998). Cultural Memory and Biodiversity. University of Arizona Press: Tuscon, AZ.

Nettle, D., \& Romaine, S. (2000). Vanishing Voices: The Extinction of the World's Languages. Oxford: Oxford University Press.

Niamir-Fuller, M. (1998). The resilience of pastoral herding in Sahelian Africa. In F. Berkes, C. Folke \& J. Colding (Eds.), Linking Social and Ecological Systems: Management Practices and Social Mechanisms for Building Resilience. Cambridge: Cambridge University Press.

Niroa, J. (2002). Reflections on Vanuatu's Education System. In F. Pene, A. Taufe'ulungaki \& C. Benson (Eds.), Tree of Opportunity: Re-tbinking Pacific Education. Suva: Institute of Education, University of the South Pacific.

Niroa, J. (2004). Why we need to re-think Vanuatu education. In K. Sanga, J. Niroa, K. Matai \& L. Crowl (Eds.), Re-thinking Vanuatu Education Together. Port Vila: University of the South Pacific.

Nirua, J.-P. (2004). A mountain of opportunity and hope. In K. Sanga, J. Niroa, K. Matai \& L. Crowl (Eds.), Re-thinking Vanuatu education together. Port Vila: University of the South Pacific.

Nyhus, P. J., Sumianto, \& Tilson, R. (2003). Wildlife knowledge among migrants in southern Sumatra, Indonesia: Implications for conservation. Environmental Conservation, 30(2), 192-199.

Odochao, J., Nakashima, D., \& Vaddhanaphuti, C. (2006). An education rooted in two worlds: the Karen of northern Thailand. International Social Science Journal, 58(187), 117-120.

Ohmagari, K., \& Berkes, F. (1997). Transmission of indigenous knowledge and bush skills among the Western James Bay Cree women of Subarctic Canada. Human Ecology, 25(2), 197-223.

Ostrom, E. (1990). Governing the commons: The evolution of institutions for collective action. Cambridge: Cambridge University Press. 
Ostrom, E., Janssen, M., \& Anderies, J. M. (2007). Going beyond panaceas. Proceedings of the National Academy of Sciences, 104(39), 15176-15178.

Paviour-Smith, M. (2005). Is it Aulua or education dressed up in 'kastom'? An ongoing report of the ongoing negotiation of literacy and identity in a ni-Vanuatu community. Current Issues in Language Planning, 6(2), 224-238.

Pelling, M., \& Uitto, J. (2001). Small island developing states: Natural disaster vulnerability and global change. Global Environmental Change Part B: Environmental Hazards, 3(2), 49-62.

Pene, F., Taufe'ulungaki, A., \& Benson, C. (Eds.). (2002). Re-thinking Pacific Education. Suva: Institute of Education, University of the South Pacific.

Peroni, N., \& Hanazaki, N. (2002). Current and lost diversity of cultivated varieties, especially cassava, under swidden cultivation systems in the Brazilian Atlantic forest. Agriculture, Ecosystems \& Environment, 92, 171-183.

Perrings, C. (2007). Future challenges. Proceedings of the National Academy of Sciences, 04(39), 15179-15180.

Phillips, O., \& Gentry, A. (1993). The useful plants of Tambopata, Peru: II. Additional hypothesis testing in quantitative ethnobotany. Economic Botany, 47(1), 33-43.

Pierce, E. (2010). Possession syntax in Unua DPs. In R. Mercade, P. E \& T. L (Eds.), Austronesian and Theoretical Linguistics. Amsterdam: Benjamins.

Pierotti, R., \& Wildcat, D. (2000). Traditional ecological knowledge: The third alternative (commentary). Ecological Applications, 10(5), 1333-1340.

Pilgrim, S., Samson, C., \& Pretty, J. (2009). Rebuilding Lost Connections: How Revitalisation Projects Contribute to Cultural Continuity and Improve the Environment. University of Essex, UK: Interdisciplinary Centre for Environment and Society Occasional Paper 2009-01.

Pilgrim, S., Smith, D., \& Pretty, J. (2007). A cross-regional assessment of the factors affecting ecoliteracy: Implications for policy and practice. Ecological Applications, 17, 1742-1751.

Plants for a Future. (2011). Main Page. Retrieved 2nd September, 2011, from http://www.pfaf.org/user/default.aspx

Plotkin, M. (1993). Tales of a Shaman's Apprentice: An Ethnobotanist Searches for New Medicines in the Amazon Rainforest. New York: Viking Penguin.

Pollard, D., Almond, R., Duncan, E., Grooten, M., Hadeed, L., Jeffries, B., et al. (2010). Living Planet Report 2010: Biodiversity, Biocapacity, and Development. Gland, Switzerland: WWF International.

Posey, D. (2000). Ethnobiology and ethnoecology in the context of national laws and international agreements affecting indigenous and local knowledge, traditional resources and intellectual 
property rights. In R. Ellen, P. Parkes \& A. Bicker (Eds.), Indigenous Environmental Knowledge and its Transformations: Critical Anthropological Perspectives. London and New York: Routledge.

Posey, D. (2001). Biological and cultural diversity: The inextricable, linked by language and politics. In L. Maffi (Ed.), On Biocultural Diversity: Linking Language, Knowledge and the Environment. Washington DC: Smithsonian Institution Press.

Posey, D., \& Dutfield, G. (1996). Beyond intellectual property: Toward traditional resource rights for indigenous peoples and local communities. Ottawa: International Development Research Centre.

Pretty, J. (2003). Agri-culture: Reconnecting people, land, and nature. London: Earthscan.

Pretty, J. (2011). Interdisciplinary progress in approaches to address social-ecological and ecocultural systems. Environmental Conservation, 38(2), 127-139.

Pretty, J., \& Smith, D. (2004). Social capital in biodiversity conservation and management. Conservation Biology, 18(3), 631-639.

Prober, S., O'Connor, M., \& Walsh, F. (2011). Australian Aboriginal people's seasonal knowledge: A potential basis for shared understanding in environmental management. Ecology and Society, 16(2), $12-28$.

Quinlan, M. (2005). Considerations for collecting freelists in the field: Examples from ethnobotany. Field Methods, 17, 219-237.

Quinlan, M., \& Quinlan, R. (2007). Modernisation and medicinal plant knowledge in a Caribbean horticultural village. Medicinal Antbropology Quarterly, 21 (2), 169-192.

Rahmann, A. (2000). Development of an integrated Traditional and Scientific Knowledge base: A mechanism for accessing, benefit-sharing and documenting Traditional Knowledge for sustainable socio-economic development and poverty alleviation. Paper presented at the UNCTAD Expert Meeting on Systems and National Experiences for Protecting Traditional Knowledge, Innovations and Practices.

Redford, K., \& Sanderson, S. (2000). Extracting humans from nature. Conservation Biology 14(5), 1362-1364.

Redford, K., \& Stearman, A. (1993). Forest-dwelling Native Amazonians and the conservation of biological diversity: Interests in common or in collision? Conservation Biology 7(2), 248-255.

Regenvanu, R. (1999). Afterword: Vanuatu perspectives on research. Oceania, 70, 98-100.

Regenvanu, R. (2002). A New Vision of Education. Paper presented at the National Education Conference: Port Vila.

Regenvanu, R. (2004). Education initiatives of the Vanuatu Cultural Centre. In K. Sanga, J. Niroa, K. Matai \& L. Crowl (Eds.), Re-thinking V anuatu Education Together. Port Vila: University of the South Pacific. 
Regenvanu, R. (2005). The changing face of 'custom' in Vanuatu. People and Culture in Oceania, 20(Special edition).

Regenvanu, R. (2006, 24 April). Panel session on indigenous and local communities' concerns and experiences in protecting their traditional knowledge and cultural expressions. Paper presented at the 9th Session of the Intergovenmental Committee on Intellectual Property and Genetic Resources, Traditional Knowledge and Folklore (IGC) of the World Intellectual Property Organisation (WPO), Geneva, Switzerland.

Regenvanu, R. (2007a). Making Policy to Support Living Cultures: A Case Study in 'Mainstreaming Culture' from Vanuatu. Paper presented at the conference Islands at a Crossroads: Cultural diversities in Small Island Developing States: Seychelles.

Regenvanu, R. (2007b, 11 February). The year of the traditional economy - What is it all about? The Independent, pp. 18-19.

Regenvanu, R. (2008). Issues with land reform in Vanuatu. Journal of South Pacific Law 12(8), 63-67.

Reichel-Dolmatoff, G. (1976). Cosmology as ecological analysis: A view from the rain forest. Man, 11, 307-318.

Reyes-Garcia, V. (2001). Indigenous people, ethnobotanical knowledge, and market economy: A case study of the Tsimane Amerindians in Lowland Bolivia. University of Florida, Gainesville.

Reyes-García, V., Broesch, J., Calvet-Mir, L., Fuentes-Peláez, N., McDade, T. W., Parsa, S., et al. (2009). Cultural transmission of ethnobotanical knowledge and skills: an empirical analysis from an Amerindian society. Evolution and Human Behavior, 30(4), 274-285.

Reyes-Garcia, V., Kightley, E., Ruiz-Mallen, I., Fuentes-Pelaez, N., Demps, K., Huanca, T., et al. (2010). Schooling and local environmental knowledge: Do they compliment or substitute each other? International Journal of Educational Development, 30, 305-313.

Reyes-Garcia, V., Marti, N., McDade, T., Tanner, S., \& Vadez, V. (2007). Concepts and methods in studies measuring individual ethnobotanical knowledge. Journal of Ethnobiology, 27(2), 182-203.

Reyes-Garcia, V., Vadez, V., Byron, E., Apaza, L., Leonard, W., Perez, E., et al. (2005). Market economy and the loss of folk knowledge of plant uses: Estimates from the Tsimane' of the Bolivian Amazon. Current Anthropology, 46(4), 651-656.

Reyes-Garcia, V., Vadez, V., Huanca, T., Leonard, W., \& McDade, T. (2007). Economic development and local ecological knowledge: A deadlock? Quantitative research from a native Amazonian society. Human Ecology: An Interdisciplinary Joumal, 35(3), 371-377.

Reyes-Garcia, V., Vadez, V., Tanner, S., Huanca, T., Leonard, W., \& McDade, T. (2006). Measuring what people know about the environment: A review of quantitative studies. Tsimane Amazonian Panel Study Working Paper, \#21. Retrieved from 
http://people.brandeis.edu/ rgodoy/working\%20papers/TAPS-WP-21-TEKMETHODS-Jan2006.pdf

Reyes-Garcia, V., Vadez, V., Tanner, S., McDade, T., Huanca, T., \& Leonard, W. (2006). Evaluating indices of traditional ecological knowledge: A methodological contribution. Joumal of Ethnobiology and Ethnomedicine, 2(21).

Rio, K. (2010). Handling sorcery in a state system of law: Magic, violence, and kastom in Vanuatu. Oceania, 80, 182-197.

Rocheleau, D. (1991). Gender, ecology, and the science of survival: Stories and lessons from Kenya. Agriculture and Human Values, 8, 156-165.

Rogoff, B. (1981). Adults and peers as agents of socialisation: A highland Guatemalan profile. Ethos, 9(1), 18-36.

Romaine, S. (2008). Linguistic diversity, sustainability and the future of the past. In K. King, N. SchillingEste, L. Fogle, J. Lou \& B. Soukup (Eds.), Sustaining Linguistic Diversity: Endangered and Minority Languages and Language V arieties. Washington DC: Georgetown University Press.

Romney, A., Boyd, J., Moore, C., Batchelder, W., \& Brazill, T. (1996). Culture as shared cognitive representations. Proceedings of the National Academy of Sciences, USA, 93, 4699-4705.

Rosillo-Cale, F., \& Woods, J. (2003). Individual country biomass resource assessment profiles for Fiji, Kiribati, Samoa, Tonga, Tuvalu \& Vanuatu: SOPAC Technical Report 364.

Ross, N. (2002a). Cognitive aspects of intergenerational change: Mental models, cultural change, and environmental behaviour among the Lacandon Maya of Southern Mexico. Human Organization, $61(2), 125-138$

Ross, N. (2002b). Lacandon Maya intergenerational change and the erosion of folk biological knowledge. In J. R. Stepp, F. S. Wyndham \& R. K. Zarger (Eds.), Ethnobiology and Biocultural Diversity. Athens, Georgia: The International Society of Ethnobiology.

Ruddle, K. (1993). The transmission of traditional ecological knowledge. In J. T. Inglis (Ed.), Traditional ecological knowledge: Concepts and cases. Ottawa: International Program on Traditional Ecological Knowledge and the International Development Research Centre.

Ruddle, K., \& Chesterfield, R. (1977). Education for Traditional Food Procurement in the Orinoco Delta. Berkeley: University of California Press.

Ruiz-Mallen, I., Barraza, L., Bodenhorn, B., de la Paz Ceja-Adame, M., \& Reyes-Garcia, V. (2010). Contextualising learning through the participatory construction of an environmental education programme. International Journal of Science Education, 13(1), 1755-1770. 
Ruiz-Mallen, I., Barraza, L., Bodenhorn, B., \& Reyes-Garcia, V. (2009). Evaluating the impact of an environmental education programme: An empirical study in Mexico. Environmental Education Research, 15(3), 371-387.

Salick, J., Cellinese, N., \& Knapp, S. (1997). Indigenous diversity of Cassava: Generation, maintenance, use and loss among the Amuesha, Peruvian upper Amazon. Economic Botany, 51(1), 6-19.

Salmon, E. (2000). Kincentric ecology: Indigenous perceptions of the human-nature relationship. Ecological Applications, 10(5), 1327-1332.

Sanga, K. (2002). Beyond Access and Participation: Challenges facing Pacific Education. In F. Pene, A. Taufe'ulungaki \& C. Benson (Eds.), Tree of Opportunity: Re-thinking Pacific Education. Suva: Institute of Education, University of the South Pacific.

Sanga, K., \& Niroa, J. (2004). First steps and small beginnings in Vanuatu educational research. In K. Sanga, J. Niroa, K. Matai \& L. Crowl (Eds.), Re-thinking V anuatu education together. Port Vila: University of the South Pacific

Sanga, K., Niroa, J., Matai, K., \& Crowl, L. (Eds.). (2004). Re-thinking V anuatu Education Together. Port Vila: University of the South Pacific.

Scheyvens, R., \& Storey, D. (Eds.). (2003). Development Fieldwork: A Practical Guide. London: Sage Publications.

Schilderman, T. (2004). Adapting traditional shelter for disaster mitigation and reconstruction: Experiences with community-based approaches. Building Research \& Information, 32(5), 414-426.

Schwartzman, S., Nepstad, D., \& Moreira, A. (2000). Arguing tropical forest conservation: People versus parks. Conservation Biology 14(5), 1370-1374.

Secretariat of the Pacific Community. (2010). Valuing Culture in Oceania. Noumea, New Caledonia: Prepared by Synexe Consulting Ltd.

Seitz, S. (1998). Coping strategies in an ethnic minority group: The Aeta of Mt Pinatubo. Disasters, 22(1), 76-90.

Setalaphruk, C., \& Price, L. (2007). Children's traditional ecological knowledge of wild food resources: A case study in a rural village in Northeast Thailand. Journal of Ethnobiology and Ethnomedicine, 3(33).

Shipman, T. (2008). Ples blong lanwis: Vernacular education in Vanuatu from a community perspective.

Paper presented at the ASAO Annual Meeting.

Shiva, V. (1988). Staying Alive: Women, Ecology, and Development. London: Zed Press.

Shiva, V. (2006). Monocultures of the mind. In J. Henry (Ed.), Creative Management and Development. London SAGE. 
Shukla, S., \& Gardner, J. (n.d.). The role of traditional ecological knowledge in education for communitybased resource management Available from http://dlc.dlib.indiana.edu/archive/00001488/00/Shukla_Role_040511_Paper223.pdf

Siegal, J. (1996). Vernacular Education in the South Pacific. Canberra: Australian Agency for International Development.

Siegel, H. (2002). Multiculturalism, universalism and science education: In search of common ground. Science Education, 86, 803-820.

Sillitoe, P. (1998). The development of indigenous knowledge: A new applied anthropology. Current Anthropology, 39(2), 223-253.

Sillitoe, P. (2002). Globalizing indigenous knowledge. In P. Sillitoe, A. Bicker \& J. Pottier (Eds.), Participating in development: Approaches to indigenous knowledge. London: Routledge.

Sillitoe, P. (2007). Local science vs global science: An overview. In P. Sillitoe (Ed.), Local science vs. global science: Approaches to Indigenous Knowledge in international development. New York: Berghahn Books.

Sillitoe, P. (2010). Trust in development: Some implications of knowing in indigenous knowledge. Journal of the Royal Anthropological Institute, 16(1), 12-30.

Singh, R. K., Pretty, J., \& Pilgrim, S. (2010). Traditional knowledge and biocultural diversity: Learning from tribal communities for sustainable development in northeast India. Journal of Environmental Planning and Management, 53(4), 511-533.

Smith, D., Pilgrim, S., \& Cullen, L. (2007). Coral reefs and people. In J. Pretty, A. Ball, T. Benton, J. Guivant, D. Lee, D. Orr, M. Pfeffer \& H. Ward (Eds.), The SAGE bandbook of environment and society. London SAGE Publications Ltd.

Smith, J. (1993). Using ANTHROPAC 3.5 and a spreadsheet to compute a freelist salience Index. Cultural Anthropology Methods Letter, 5, 1-3.

Smith, L. T. (1999). Decolonising Methodologies: Research and Indigenous Peoples. Dunedin: University of Otago Press.

Smith, L. T. (2005). On tricky ground: Researching the native in the age of uncertainty. In N. Denzin \& Y. Lincoln (Eds.), The SAGE Handbook of Qualitative Research (3 ed). Thousand Oaks: SAGE.

Snively, G., \& Corsiglia, J. (2001). Discovering indigenous science: Implications for science education. Science Education, 85(1), 6-34.

Spriggs, M. (1985). 'A school in every district': the cultural geography of conversion on Aneityum, southern Vanuatu The Journal of Pacific History, 20(1), 23-41.

Spriggs, M. (1996). The archaeology of Vanuatu in a Pacific perspective. In J. Bonnemaison, K. Huffman, C. Kaufmann \& D. Tryon (Eds.), Arts of V anuatu. Honolulu: University of Hawaii Press. 
SPSS Inc. (2008). SPSS for Windows 17.0. Chicago: SPSS Inc.

Stagegaard, J., Sørensen, M., \& Kvist, L. P. (2002). Estimations of the importance of plant resources extracted by inhabitants of the Peruvian Amazon flood plains. Perspectives in Plant Ecology, Evolution and Systematics, 5(2), 103-122.

Stahl, J. (2004). Vernacular education in Vanuatu: A fond memory of a past generation or hope for a new? In K. Sanga, J. Niroa, K. Matai \& L. Crowl (Eds.), Re-thinking Vanuatu Education Together Port Vila: University of the South Pacific.

Stanley, W., \& Brickhouse, N. (2001). Teaching sciences: The multicultural question revisited. Science Education, 85, 35-49.

Steffen, W., Crutzen, P. J., \& McNeill, J. R. (2007). The Anthropocene: Are humans now overwhelming the great forces of nature? Ambio, 36(8), 614-621.

Stephenson, J., \& Moller, H. (2009). Cross-cultural environmental research and management: Challenges and progress. Journal of the Royal Society of New Zealand, 39(4), 139-149.

Stepp, J. R., \& Moerman, D. E. (2001). The importance of weeds in ethnopharmacology. Journal of Ethnopharmacology, 75(1), 19-23.

Sternberg, R., Nokes, C., Geissler, P. W., Prince, P., Okatcha, F., Bundy, D., et al. (2001). The relationship between academic and practical intelligence: A case study in Kenya. Intelligence, 29, 401-418.

Straume, A. (2009). Medicine of the Past and Present: A Study in Medical Knowledge and Practice in a Solomon Islands Village. University of Bergen, Bergen.

Stross, B. (1973). Acquisition of botanical terminology by Tzeltal children. In M. S. Edmonson (Ed.), Meaning in Mayan Languages (pp. 107-141). The Hague: Mouton.

Sundar, N. (2002). "Indigenise, nationalise, spiritualise" - an agenda for education? International Social Science Journal, 54(173), 373-383.

Super, C., \& Harkness, S. (1986). The developmental niche: A conceptualization at the interface of child and culture. International Joumal of Behavioural Development, 9(4), 545-569.

Super, C., \& Harkness, S. (2002). Cultural structures the environment for development. Human Development, 45(4), 270-274.

Sutherland, W. J. (2003). Parallel extinction risk and global distribution of languages and species. Nature, 423(6937), 276(274).

Suzuki, D., \& McConnell, A. (1997). The Sacred Balance: Rediscovering Our Place in Nature. Vancouver: Greystone.

Tacconi, L., \& Bennet, J. (1993). The Forests of V anuatu: An Overview of their Environmental and Economic Status. Sydney: Vanuatu Forest Conservation Research Reports: University of New South Wales. 
Taufe'ulungaki, A. (2002). Pacific Education at the Crossroads: Are there alternatives? In F. Pene, A.

Taufe'ulungaki \& C. Benson (Eds.), Tree of Opportunity: Re-thinking Pacific Education. Suva: Institute of Education, University of the South Pacific.

Taylor, P., \& Mulhall, A. (2001). Linking learning environments through agricultural experience Enhancing the learning process in rural primary schools. International Journal of Educational Development, 21, 135-148.

Teasdale, B. (1995). Education and Culture: An Introduction. Prospects, XXV(4).

Teasdale, B., \& Ma Rhea, Z. (Eds.). (2000). Local Knowledge and Wisdom in Higher Education. Oxford: IAU Press, Pergamon.

Teasdale, B., Tokai, E., Puamau, P., \& Singh, M. (2005, 2005). Culture, Literacy and Livelihoods: Reconceptualising the Reform of Education in Oceania. Paper presented at the Literacy and Livelihoods: Learning for Life in a Changing World, Vancouver.

Teasdale, G. R. (2005). The Big Picture: International Perspectives on the Reform of Education. Paper presented at the Vanuatu Institute of Teacher Education National Workshop: Port Vila.

Terborgh, J. (2000). The fate of tropical forests: A matter of stewardship. Conservation Biology 14(5), 13581361.

Thaman, K. H. (2000). Toward a new pedagogy: Pacific cultures in higher education. In B. Teasdale \& Z. Ma Rhea (Eds.), Local Knowledge and Wisdom in Higher Education. Oxford: Elsevier Science Ltd.

Thaman, K. H. (2002). Towards cultural democracy in Pacific education: An imperative for the 21st century. In F. Pene, A. Taufe'ulungaki \& C. Benson (Eds.), Tree of Opportunity: Re-thinking Pacific Education. Suva: Institute of Education, University of the South Pacific.

TKDL. (2011). Indian Traditional Knowledge Digital Library. Retrieved 2nd September, 2011, from http://www.tkdl.res.in/tkdl/langdefault/common/AboutTKDL.asp?GL=\#Present

Toledo, V. M. (2001). Biocultural diversity and local power in Mexico: Challenging globalization. In L. Maffi (Ed.), On Biocultural Diversity: Linking Language, Knowledge, and the Environment. Washington: Smithsonian Institution.

Toledo, V. M. (2002). Ethnoecology: A conceptual framework for the study of indigenous knowledge of nature. In J. R. Stepp, F. S. Wyndham \& R. K. Zarger (Eds.), Ethnobiology and biocultural diversity. Athens, Georgia: The International Society of Ethnobiology.

Tompkins, A., \& Adger, W. N. (2004). Does adaptive management of natural resources enhance resilience to climate change? Ecology and Society, 9(2). Retrieved from http://www.ecologyandsociety.org/vol9/iss2/art10/

Tonkinson, R. (1982). National identity and the problem of kastom in Vanuatu. Mankind, 13(4), 306-315. 
Tryon, D. (1976). New Hebrides Languages: An Internal Classification. Canberra: Pacific Institute of Linguistics

Tryon, D. (1979). The language situation in the New Hebrides. In S. A. Wurm (Ed.), New Guinea and Neighbouring Areas. The Hague Mouton.

Tryon, D. (1996). Dialect chaining and the use of geographical space. In J. Bonnemaison, K. Huffman, C. Kaufmann \& D. Tryon (Eds.), Arts of Vanuatu. Honolulu: University of Hawaii Press.

Tryon, D. (1999). Ni-Vanuatu research and researchers. Oceania, 70, 9-15.

Tuiono, T. (2011). Contribution to conference workshop: "Communications Tools for Sharing Power". Paper presented at the IUCN Sharing Power Conference.

Turner, N., \& Berkes, F. (2006a). Coming to understanding: Developing conservation through incremental learning in the Pacific Northwest. Human Ecology 34(4), 495-513.

Turner, N., \& Berkes, F. (2006b). Developing resource management and conservation. Human Ecology 34(4), 475-478.

Turner, N., Davidson-Hunt, I., \& O'Flaherty, M. (2003). Living on the edge: Ecological and cultural edges as sources of diversity for social-ecological resilience Human Ecology, 31(3), 439-461.

U.S. Department of State. (2011). Background note: Vanuatu. Retrieved 25th October, 2011, from http://www.state.gov/r/pa/ei/bgn/2815.htm

Ugent, D. (2000). Medicines, myths, and magic: The folk healers of a Mexican market. Economic Botany, 54, 427-438.

UNESCO. (2011). Traditional Knowledge World Bank. Retrieved 2nd September, 2011, from http://www.tkwb.org/web/?page_id=4

UNFCCC. (2011). UNFCCC database on local coping strategies. Retrieved 25th March, 2011, from http://maindb.unfccc.int/public/adaptation/

UNICEF. (2003). Ensuring the Rights of Indigenous Children. Washington DC: Innocenti Research Centre, United Nations Children's Fund.

United Nations. (2009). World urbanization prospects. Accessed 24/06/2011 at http://esa.un.org/unpd/wup/index.htm: United Nations, Department of Economic and Social Affairs.

United Nations. (2011). Culture and development: Report of the Director-General of the UNESCO: United Nations General Assembly.

Van Eijck, M., \& Roth, W. (2007). Keeping the local local: Recalibrating the status of science and Traditional Ecological Knowledge (TEK) in education. Science Education, 91(6), 926-947. 
van Etten, J. (2006). Changes in farmers' knowledge of maize diversity in highland Guatemala, 1927/372004. Journal of Ethnobiology and Ethnomedicine, 2(12).

Van Trease, H. (1987). The Politics of Land in Vanuatu. Suva, Fiji: University of the South Pacific.

Vandebroek, I. (2010). The dual intracultural and intercultural relationship between medicinal plant knowledge and consensus. Economic Botany, In press, 1-15.

Vanuatu Cultural Centre. (2005). Vanuatu Young People's Project. Retrieved 13th October, 2011, from http://www.vanuatuculture.org/site-bm2/ypp/vanuatu-young-peoples-pro.shtml

Vanuatu Cultural Centre. (2011). The Vanuatu Cultural Research Policy. Retrieved 4th September, 2011, from http://www.vanuatuculture.org/site-bm2/research/050520_culturalresearchpolicy.shtml

Vanuatu Ministry of Education. (2010). Vanuatu National Curriculum Statement (Edited Draft). Port Vila: Ministry of Education.

Vanuatu National Statistics Office. (1999). National population and housing census. Port Vila: Vanuatu Government.

Vanuatu National Statistics Office. (2006). National Agriculture Census. Port Vila: Vanuatu Government.

Vanuatu National Statistics Office. (2009). National Population and Housing Census: Basic Tables Report Volume One. Port Vila: Republic of Vanuatu.

Vari-Bogiri, H. (2005). A sociolinguistic survey of Araki: A dying language in Vanuatu. Journal of Multilingual Multicultural Development, 26(1).

Victoria University of Wellington. (2009). Human Ethics Policy. Wellington: Research Policy Group, Victoria University of Wellington.

Vigestad, O. (1984). Waiting for paradise: On the 'kastom' movement, a naticistic, politico-religious movement on Tanna, Vanuatu. Oslo, Oslo.

Voeks, R. (2004). Disturbance pharmacopoeias: Medicine and myth from the humid tropics. Annals of the Association of American Geographers, 94(4), 868-888.

Voeks, R. (2007). Are women reservoirs of traditional plant knowledge? Gender, ethnobotany and globalization in northeast Brazil. Singapore Journal of Tropical Geography, 28, 7-20.

Voeks, R., \& Leony, A. (2004). Forgetting the forest: Assessing medicinal plant erosion in Eastern Brazil. Economic Botany, 58 (Supplement), S294-S306.

Voeks, R., \& Nyawa, S. (2001). Healing flora of the Brunei Dusun. Bormeo Research Bulletin, 32, 178-195.

Voeks, R., \& Sercombe, P. (2000). The scope of hunter-gatherer ethnomedicine. Social Science and Medicine, $50,1-12$.

Vygotsky, L. (1978). Mind in Society. Cambridge, MA: Harvard University Press. 
Warren, D. (1995). Comments on article by Arun Agrawal. Indigenous Knowledge and Development Monitor, $4(1)$.

Warren, D. (2001). The role of the global network of indigenous knowledge resource centres in the conservation of cultural and biological diversity. In L. Maffi (Ed.), On Biocultural Diversity: Linking Language, Knowledge, and the Environment. Washington DC: Smithsonian Insitution Press.

Warren, D., Slikkerveer, L. J., \& Brokensha, D. (Eds.). (1995). The Cultural Dimension of Development: Indigenous Knowledge Systems. London: Intermediate Technology Publications.

Warrick, O. (2009). Climate Change and Social Change: Vulnerability and Adaptation in Rural Vanuatu. Paper presented at the 11th Pacific Science Inter-Congress.

Weightman, B. (1989). Agriculture in Vanuatu: A Historical Review. Port Vila: British Friends of Vanuatu.

Wenzel, G. (1999). Traditional ecological knowledge and Inuit: Reflections on TEK research and ethics. Arctic 52(2), 113-124.

Westley, F., Carpenter, S., Brock, W., Holling, C. S., \& Gunderson, L. (2002). Why systems of people and nature are not just social and ecological systems. In L. Gunderson \& C. S. Holling (Eds.), Panarchy: Understanding Transformations in Human and Natural Systems. Washington: Island Press.

Wheatley, J. I. (1992). A Guide to the Common Trees of Vanuatu: With Lists of their Traditional Uses \& ni-Vanuatu names. Port Vila: Department of Forestry.

Williams, V., Witkowski, E., \& Balkwill, K. (2007). The use of incidence-based species richness estimators, species accumulation curves, and similarity measures to appraise ethnobotanical inventories in South Africa. Biodiversity and Conservation, 16, 2495-2513.

World Health Organisation. (2008). Fact Sheet on Traditional Medicine. Retrieved 26/10, 2010, from http://www.who.int/mediacentre/factsheets/fs134/en/

World Intellectual Property Organisation. (2011). Portal of Online Databases and Registries of Traditional Knowledge and Genetic Resources. Retrieved 2nd September, 2011, from http://www.wipo.int/tk/en/databases/tkportal/

Zarger, R. K. (2002). Acquisition and transmission of subsistence knowledge by Q'echi' Maya in Belize. In J. R. Stepp, F. S. Wyndham \& R. K. Zarger (Eds.), Ethnobiology and Biocultural Diversity. Athens, Georgia: The International Society of Ethnobiology.

Zarger, R. K., \& Stepp, J. R. (2004). Persistence of botanical knowledge among Tzeltal Maya children. Current Anthropology, 45(3), 413 - 418.

Zent, S. (1999). The quandary of conserving ethnoecological knowledge: A Piaroa example. In T. Gragson \& B. Blount (Eds.), Ethnoecology: Knowledge, Resources and Rights. Athens, Georgia: The University of Georgia Press. 
Zent, S. (2001). Acculturation and ethnobotanical knowledge loss among the Piaroa of Venezuela: Demonstration of a quantitative method for the empirical study of TEK change. In L. Maffi (Ed.), On Biocultural Diversity: Linking Language, Knowledge, and the Environment. Washington and London: Smithsonian Institution Press.

Zent, S. (2009). Traditional ecological knowledge (TEK) and biocultural diversity: A close-up look at linkages, delearning trends \& changing patterns of transmission. In P. Bates, M. Chiba, S. Kube \& D. Nakashima (Eds.), Learning and Knowing in Indigenous Societies Today. Paris: UNESCO.

Zent, S., \& Maffi, L. (2009). Final Report on Indicator No. 2: Methodology for Developing a Vitality Index of Traditional Environmental Knowledge (VITEK) for the Project "Global Indicators of the Status and Trends of Linguistic Diversity and Traditional Knowledge". Terralingua: Accessed 3rd of March 2009 from http://terralingua.org/projects/vitek/VITEK_Report.pdf.

Zent, S., \& Zent, E. L. (2004). Ethnobotanical convergence, divergence, and change among the Hoti of the Venezuelan Guyana. In J. S. Carlson \& L. Maffi (Eds.), Ethnobotany and the Conservation of Biocultural Diversity. New York: New York Botanical Garden Press.

Zent, S., \& Zent, E. L. (2007). On Biocultural diversity from a Venezuelan perspective: Tracing the interrelations among biodiversity, culture change, and legal reforms. In C. McManis (Ed.), Biodiversity and the Law: Intellectual Property, Biotechnology, and Traditional Knowledge. London: Earthscan 


\section{Appendices}

\subsection{Ethics approval (2008)}

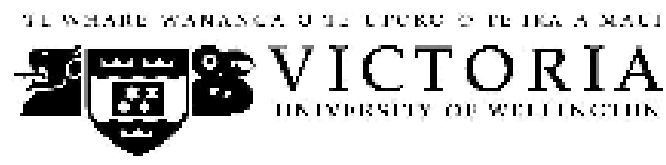

\section{MEMORANDUM}

Phone 0-4-4635676

Fax 0-4-4635209

Email Allison.kirkmaniovuw.ac.nz.

\begin{tabular}{l|l}
\hline TO & Joe McCarter \\
\hline COPY TO & Dr Michael Gavin, Supervisor \\
\hline FROM & Dr Allison Kirkman, Convener, Human Ethics Committee \\
\hline
\end{tabular}

\begin{tabular}{l|l}
\hline DATE & March 19, 2008 \\
\hline PAGES & 1
\end{tabular}

\begin{tabular}{l|l}
\hline SUBJECT & $\begin{array}{l}\text { Ethics Approval: No 15452, Traditional ecological knowledge } \\
\text { change and education: a community perspective from } \\
\text { Malakula, Vanuatu }\end{array}$
\end{tabular}

Thank you for your application for ethical approval, which has now been considered by the Standing Committee of the Human Ethics Committee.

Your application has been approved from the above date and this approval continues until 25 February 2009. If your data collection is not completed by this date you should apply to the Human Ethics Committee for an extension to this approval.

Best wishes with the research.

Allison Kirkman

Convener 


\title{
11.2 Information and informed consent forms (2008)
}

\subsubsection{Community interviewees (information sheet and informed consent)}

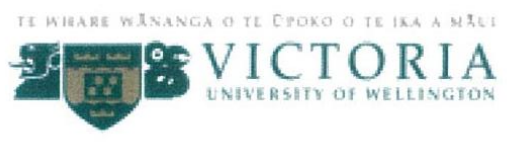

Consent and Information for Community Interviews

\author{
Joe McCarter
}

- I am doing work for one project with my university in collaboration with the Vanuatu Cultural Centre and Dr Michael Gavin. My project will investigate community views of traditional ecological knowledge loss and education on Malekula.

- In this study I want to talk with people about traditional ecological knowledge; how it is changing; what people think about this; and how the school system affects it. I am working with the VKS who are looking at making local education modules for TEK, and as part of the bigger project my supervisor and I will produce a resource booklet on TEK for Malekula.

- I am doing my research by talking with people all over Malekula and with government people in Port Vila. I would like to talk with people when it suits them, and get their opinions on how TEK is changing and why.

- I would like to talk with you about TEK. The interview should not last for more than one hour, and will probably be less.

- Do you have any questions about what I am doing? If you do not want to talk about TEK, that is fine, we will not continue.

- As part of my university rules, I need your consent to collect your opinions. I will now read some things about what I will do with the things you say, then will ask you to answer some questions.

- I will not use your name in my report. Also, all information collected from all my interviews will be confidential, and all data will be only able to be seen by me, my supervisor, and some people cleared by the National Cultural Council.

- All written material will be kept in a locked file, and all electronic material will be password protected.

- The results collected will be reported in my thesis, and will be potentially presented in academic journals and conferences.

- During the course of the interview, you can withdraw from the interview or refuse to answer any question(s) at any time. You may request that the transcript of your interview be destroyed and not used in the study, for any reason. Your must tell me of your withdrawal no more than one week after this interview.

I will now read you some statements. Please let me know if you understand and ask any questions you might have.

1. Do you understand that you are being asked to take part in an interview that will take no more than one hour? 
2. Do you understand that your name will be confidential in this project?

3. Do you understand that the data collected will not be used for any purpose other than what I have told you?

$$
\mathrm{Y} / \mathrm{N}
$$

4. Do you undersatnd that data collected in this interview may be stored securely and accessed by Joe McCarter, Dr Michael Gavin and researchers approved by the National Cultural Council?

$$
\mathrm{Y} / \mathrm{N}
$$

5. Do you understand that a summary overview of the research program will be provided to my community at the completion of Dr Michael Gavin's research program.

Do you agree to continue in this interview?

$$
\mathbf{Y} / \mathbf{N}
$$

Researcher:

I certify that this form and its attached "Information Sheet" cover letter provide a complete and accurate description of the aims and processes of this research project.

Name:

Date:

Signature:

If you have any other questions about this project, please feel free to ask me now, or contact me or my supervisor, Dr Michael Gavin, at the address or number on the card I will give you.

Tankyu tumas! 


\title{
11.2.2 Port Vila officials (information sheet)
}

\author{
年 \\ Participant Information Sheet \\ (Officials) \\ Master's Thesis Research Project: \\ "Traditional knowledge loss and education: A community perspective from Malekula, \\ Vanuatu"
}

March, 2008

\begin{tabular}{|c|c|c|}
\hline $\begin{array}{l}\text { Researcher: } \\
\text { Telephone: } \\
\text { Mobile: } \\
\text { Supervisor: } \\
\text { Email: }\end{array}$ & $\begin{array}{l}\text { Joe McCarter } \\
006443877783 \\
0064274224209 \\
\text { Dr Michael Gavin } \\
\text { michael.gavin@vuw.ac.nz } \\
\text { joe.mccarter@gmail.com }\end{array}$ & $\begin{array}{l}\text { School of Geography, Environment } \\
\text { and Earth Sciences (SGEES) } \\
\text { Victoria University of Wellington } \\
\text { PO BOX } 600 \\
\text { Wellington } 6140 \\
\text { (04) } 463-5337\end{array}$ \\
\hline
\end{tabular}

Dear Project Participant,

You are being asked to take part in an interview for my master's thesis at Victoria University of Wellington, in collaboration with the Vanuatu Cultural Centre and Dr Michael Gavin. The project will investigate community views of traditional ecological knowledge loss on Malekula, as well as investigating the creation of an education module based on local traditional knowledge. The study will try to do the following things:

a. Describe community perceptions on the status of traditional ecological knowledge and its change

b. Describe academic, non government organisation, and government perceptions of traditional ecological knowledge and its change

c. Describe perceptions of the role of the education system in the transmission and preservation of traditional ecological knowledge

d. Define the community perceptions of the necessary parts of a local education module based on traditional ecological knowledge

I am inviting you to participate in a semi-structured interview to expand my understanding of traditional knowledge loss and education on Malekula. I will ask questions about your knowledge of traditional ecological knowledge on Malekula, how it is changing, and government plans for conserving it. The questions will not be of a personal nature. The interview will last for approximately one hour and will not exceed more than one and a half hours in length.

\section{Confidentiality}

At the beginning of the interview you will be asked whether you wish your identity to remain confidential. I will explain in detail how the results of the research may be used, including the potential for publication in the form of academic papers in peer reviewed journals. The Informed Consent Form (attached) will ask you whether you require confidentiality or not, and how you wish to identify yourself in the research. If you do not require confidentiality, you will be asked to circle the relevant statement (see \#4 on the Informed Consent Form), and only then will your identity be published.

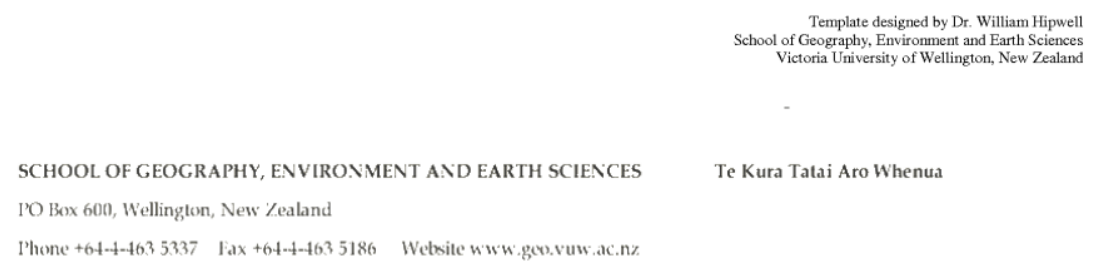


If you would like your comments to remain confidential, a letter-number code will be assigned to each interview respondent, and only this code, or other title specified by you, will appear in the published results. The real names associated with the codes will be kept securely by me, and never made public without your permission.

\section{Storage and Disposal of Data}

Access to the written and electronic material will be restricted to me, my supervisor (Dr Michael Gavin), and researchers granted access by the National Cultural Council. All written material will be kept in a locked file, and all electronic material will be password protected.

The results collected will be reported in my thesis, and will be potentially presented in academic journals and conferences. The thesis will be submitted for marking to the School of Geography, Environment and Earth Sciences, Victoria University of Wellington.

\section{Right of Withdrawal}

During the course of the interviews, you will have the right to withdraw from the interview or refuse to answer any question(s) at any time. You may request that the transcript of your interview be destroyed and not used in the study, for any reason. You must inform me of your withdrawal no more than 8 weeks after the date of the interview.

\section{Provision of Feedback}

You have the right to check the interview transcript, and will be able to provide any corrections at any time, prior to final analysis of data.

\section{Access to Research Results}

In order to ensure that the results of this research project are accessible to the participants, a summary of the completed research output will be available. You may request it by circling 'Yes' in Question 7 of the 'Informed Consent Form' (attached), or on later request (by email to joe.mccarter@gmail.com). Copies of the completed research output will also be available from the School of Geography, Environment and Earth Sciences Library, and in the Victoria University of Wellington electronic database of theses.

If you have any other questions about this project, please feel free to ask me now, or contact me or Michael Gavin later from the contact details provided on the first page of this sheet.

Thank you for participating!

Sincerely,

\section{Joe McCarter}

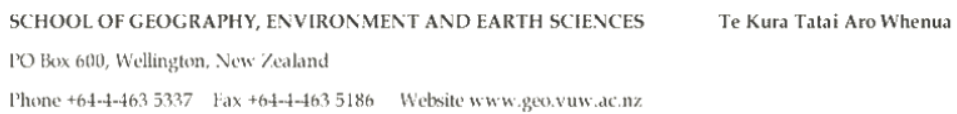




\subsubsection{Informed consent form (officials)}

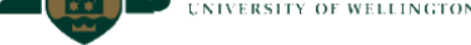

Master's Thesis Research Project:

"Traditional ecological knowledge and education: A community perspective from Malekula, Vanuatu"

INFORMED CONSENT FORM

(Officials)

\begin{tabular}{ll|l|} 
Researcher: & Joe McCarter & School of Geography, Environment \\
Telephone: & 006443877783 & and Earth Sciences (SGEES) \\
Mobile & 0064274224209 & Victoria University of Wellington \\
Supervisor & Dr Michael Gavin & PO BOX 600 \\
Tel. & 006444635195 & Wellington 6140 \\
Email: & & (04) 463-5337
\end{tabular}

\section{Introduction}

The Victoria University of Wellington Human Ethics Committee, which has approved this research project, requires that all research involves Participants who are: 1) fully informed about the nature of the research; and 2) consent to participate. This "Informed Consent Form" has been designed to meet these requirements. It is meant to ensure that research Participants and their communities are protected from any harm potentially arising from their participation in the research process.

\section{Purpose of the Study}

This study, for my master's thesis, is intended to fulfil the following objectives:

a. Describe community perceptions on the status of traditional ecological knowledge and its change

b. Describe academic, non government organisation, and government perceptions of traditional ecological knowledge and its change

c. Describe perceptions of the role of the education system in the transmission and preservation of traditional ecological knowledge

d. Define the community perceptions of the necessary parts of a local education module based on traditional ecological knowledge

Please also see the attached 'Information Sheet'.

\section{Research Format}

In addition to research being undertaken in communities on Malekula Island, I am doing semistructured interviews in Port Vila with officials concerning traditional ecological loss and education. These discussions will take place at a time and place that suits you.

I am asking for your consent to attribute opinions and data to you. No names will be attached to my results and data will not be attributable without your written consent. Questions will not be of a personal nature. Each interview will last for approximately one hour, and will last no more than one and a half hours. 
1. I agree to an audio recorded interview with Joe McCarter

Please circle one

2. I wish for my identity to remain confidential.

yes, please answer 3 . If no, please skip to 4

3. I would like to be identified as (please circle one):

a) An 'official' from the organisation I represent, or

b) A letter code, or

c) Other, please specify:.

4. I consent to information or opinions that I have given being attributed to me in any reports on this research.

$$
\mathrm{Y} / \mathrm{N}
$$

5. I understand that I will have an opportunity to correct the transcript of the interview before publication.

$$
\mathrm{Y} / \mathrm{N}
$$

6. I understand that the data I provide will not be used for any other purpose than described in the 'Information Sheet'.

$$
\mathrm{Y} / \mathrm{N}
$$

7. I understand that data collected in this interview may be stored securely and accessed by Joe McCarter, Dr Michael Gavin and researchers approved by the National Cultural Council.

$$
\mathrm{Y} / \mathrm{N}
$$

8. I would like to receive a summary of the results of this research when it is completed.

If yes, My address is:

My email is:

I agree to take part in this research

Participant:

Name:

Date:

Signature:

Researcher:

I certify that this form and its attached "Information Sheet" cover letter provide a complete and accurate description of the aims and processes of this research project. 


\subsection{Ethics approval (2009)}

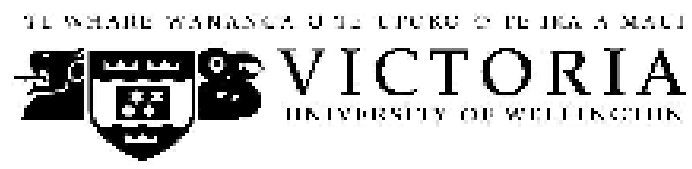

MEMORANDUM

Phone 0-4-4635676

Fax 0-4-4635209

Email Allison.kirkmanivvuw.ac.nz.

\begin{tabular}{l|l}
\hline TO & Joe McCarter \\
\hline COPY TO & Dr Michael Gavin, Supervisor \\
\hline FROM & Dr Allison Kirkman, Convener, Hurnan Ethics Committee \\
\hline
\end{tabular}

\begin{tabular}{l|l}
\hline DATE & May 17,2009 \\
\hline PAGES & 1
\end{tabular}

SUBJECT

Ethics Approval: No 16500, Traditional ecological knowledge and climate change adaptation on Malekula Island, Vanuatu.

Thank you for your application for ethical approval, which has now been considered by the Standing Committee of the Human Ethics Committee.

Your application has been approved from the above date and this approval continues until 31 January 2010. If your data collection is not completed by this date you should apply to the Human Ethics Committee for an extension to this approval.

Best wishes with the research.

Allison Kirkman

Convener 


\subsection{Ethics approval (2009 extension)}

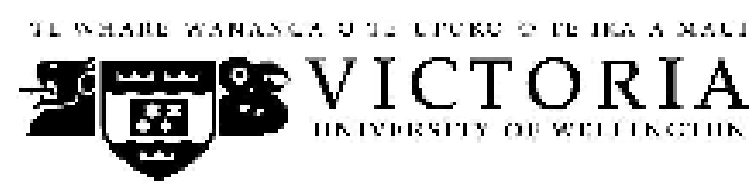

\section{MEMORANDUM}

Phone 0-4-4635676

Fax 0-4-4635209

Email Allison.kirkmanavuw.ac.nz

\begin{tabular}{l|l}
\hline TO & Joe McCarter \\
\hline COPY TO & Michael Gavin \\
\hline FROM & Dr Katherine Nelson, Acting Convener, Human Ethics Committee \\
\hline
\end{tabular}

\begin{tabular}{l|l}
\hline DATE & 18 November 2011 \\
\hline PAGES & 1 \\
\hline
\end{tabular}

SUBJECT

Ethics Approval: 16500

Traditional ecological knowledge and climate change adaptation on Malekula Island, Vanuatu

Thank you for your request to extend your ethics approval. This has now been considered and the request granted. Your application has approval until 31 November 2011.

If your data collection is not completed by this date you should apply to the Human Ethics Committee for an extension to this approval.

Best wishes with your research.

Dr Katherine Nelson

Acting Convener

Human Ethics Committee 


\subsection{Information and informed consent forms (2009-2010)}

Please note that the information sheets and consent forms included here are slightly different to those used in the field, as the research evolved after the proposal was sent to the HEC. Those used in the field did not differ in methodological approach, but were tweaked to reflect the updated objectives of this research.

\subsubsection{Information sheet (experts)}




\author{
Participant Information Sheet (experts) \\ PhD Research Project: \\ "Traditional exological knowledge and climate change adaptation on Malekela Island, \\ Vanuatu"
}

March, 2009

\begin{tabular}{|ll|l|}
\hline Researcher: & Joe McCarter & School of Geography, Environment \\
Telephone: & 006443877783 & and Earth Scie nces (SGEES) \\
Mobile: & 0064274224209 & Victoria University of Wellington \\
Supervisor: & $\begin{array}{l}\text { Dr Michael Gavin } \\
\text { michael.ga vin(Qvuw.ac.nz }\end{array}$ & FO BOx 600 \\
Email: & joe.mocarter(Q.gmail.com & (04) $463-5337$ \\
\hline
\end{tabular}

Dear Project Participant,

You are being asked to take part in a series of activities as part of my $\mathrm{FhD}$ research at Victoria University of Wellington, in collaboration with the Vanuatu Cultural Centre and Di Michael Gavin. The project will investigate perceptions of traditional knowledge change on Malakula, as well as invertigating the effects of this loss on adaptive capacity to climate change. The study will answer the following major questions:

1. What are the perceived patterns of general TEK loss actoss Mak kula?

2. How is disaster-re lated TEK changing actoss different sectors of the population in a focus community? This has two key components:

a. How is diversity of disaster re lated plant knowledge changing?

b. How are traditional practices associated with disaster relief chan ging?

3. How are these changes impacting on local ability to respond and adapt to changing climatic conditions?

4. What are local perceptions of the best way to conserve disaster-related TEK?

The study will use both quantitative and qualitative methodology including interviews, focus groups, and ethnobotanical trails.

I would like your help in three activities. First, I would like to do a listing exancise. Second, as you are an expert, I would like your help in setting up a trail in the bush to identify plants that are useful in disasters, so that I can ask other community members about them. Third, at some point in the coming weeks I would hike to hold a one-day workshop with you and other experts to discuss traditional ways of disaster relief. This will help me to identify issues I need to discuss with other community members.

I realise that this will be a signficant inconvenience to you. Therefore, I can pay you at a rate of 1000 vt. for a full days work for your time.

All the information that we discuss is entirely confidential. I will not record your name in associated with any of the information or issues that we discuss, and no user of this data will be able to attribute opinions, facts, or other information to you. 


\section{Storage and Diaposal of Dats}

Access to the written and electronic material will be restricted to me. All written material will be kept in a beked file, and all electronic material will be password protected. A.fter five years of the completion of the research, any interview material, or similar will be destroyed and the audio recordings of the interviews will be electronically wiped.

The results collected will be reported in my thesis, and will be potentially presented in academic jour nals and conferenos. The thesis will be submitted for marking to the Sctool of Geography, Environment and Earth Sciences, Victoria Uni versity of Wellington.

\section{Right of Withdrawal}

During the course of the activities, you will have the right to withdraw from the interview or refuse to answer any question(s) at any time. You may request that the transcript of your interview be destroyed and not used in the study, for a ny reason. Your must inform me of your withdrawal no more than 8 weeks after the date of the interview.

\section{Provision of Feedbact}

You have the right to chedk the interview transcript, and will be able to provide any comections at any time, prior to final analysis of data.

Community A ccess to Research Results

Dr Gavin and I will be providing to communities a resource book containing summaries of all the information that we gather during our research a round Malekula. All information gained during these interviews will be passed on to the Vanuatu Cultural Centre and will form part of their database of traditional knowledge.

If you have any other questions about this project, please feel free to ask me now, or contact me, or my supervisor, Dr Gavin, later from the contact details provided on the first page of this sheet.

Thank you for helping!

Sincerely,

\section{Joe MCCarter}




\subsubsection{Information sheets (community)}

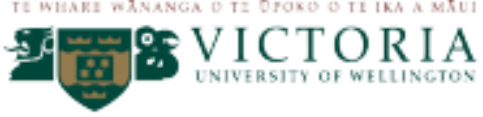

Participant Information Sheet (community participants)

PhD Reserrch Proj ect:

"Traditional ecological knowledge and climate change adaptation on Malekula Island, Vamuatu"

Mrxch, 2009

\begin{tabular}{|ll|l|}
\hline Researcher: & Joe McCarter & School of Geography, Environment \\
Telephone: & 006443877783 & and Earth Sciences (SGEES) \\
Mobile: & 0064274224209 & Victoria University of Wellington \\
Supervisor: & Dr Michael Gavin & PO BOx 600 \\
& michael.ga vin@Qvuw.ac.nz & Wellington 6140 \\
Email: & joe.mocarter(Qgemail.com & (04) $463-5337$ \\
\hline
\end{tabular}

Dear Project Participant,

You are being asked to take part in a series of activities as part of my PhD research at Victoria University of Wellington, in collaboration with the Vanuatu Cultural Centre and Dr Michael Gavin. The project will investigate perceptions of traditional knowledge change on Malakula, as well as investigating the effects of this loss on adaptive capacity to elimate change. The study will a nswer the following major questions:

1. What are the perceived patterns of general TEK loss actoss Mak kula?

2. How is disaster-re lated TEK changing actoss different sectors of the population in a focus community? This has two key components:

a. How is diversity of disaster related plant knowledge changing?

b. How are traditional practices associated with disaster relief chan ging?

3. How are these changes impacting on local ability to respond and adapt to changing climatic conditions?

4. What are local perceptions of the best way to conserve disaster-Telated TEK?

The study will use both quantitative and qualitative methodology including interviews, focus groups, and ethnobotanical trails.

I am inviting you to participate in a series of activities: a freelisting exercise, a walle-through trail, and a short interview, none of which will tale more than one hour each. We can undertale these activities at times that suit you, at any time over the coming weets. Further, towards the end of my stay I will hold some focus groups, which you are invited to attend. These will not be more than two hours each.

All the information that we discuss isentirely confidential. I will not record your name in associated with any of the information or issues that we discuss, and no u ser of this data will be able to attribute opinions, facts, or other information to you. 


\section{Storage and Diaposal of Dats}

Access to the written and electronic material will be restricted to me. All written material will be kept in a beked file, and all electronic material will be password protected. A.fter five years of the completion of the research, any interview material, or similar will be destroyed and the audio recordings of the interviews will be electronically wiped.

The results collected will be reported in my thesis, and will be potentially presented in academic jour nals and conferenos. The thesis will be submitted for marking to the Sctool of Geography, Environment and Earth Sciences, Victoria Uni versity of Wellington.

\section{Right of Withdrawal}

During the course of the activities, you will have the right to withdraw from the interview or refuse to answer any question(s) at any time. You may request that the transcript of your interview be destroyed and not used in the study, for a ny reason. Your must inform me of your withdrawal no more than 8 weeks after the date of the interview.

\section{Provision of Feedbact}

You have the right to chedk the interview transcript, and will be able to provide any comections at any time, prior to final analysis of data.

Community A ccess to Research Results

Dr Gavin and I will be providing to communities a resource book containing summaries of all the information that we gather during our research a round Malekula. All information gained during these interviews will be passed on to the Vanuatu Cultural Centre and will form part of their database of traditional knowledge.

If you have any other questions about this project, please feel free to ask me now, or contact me, or my supervisor, Dr Gavin, later from the contact details provided on the first page of this sheet.

Thank you for helping!

Sincerely,

\section{Joe MCCarter}




\subsubsection{Informed consent form}

\section{SI:}

PhD Research Project:

"Traditional ecological knowdedge and climate change adaptation on Malekela Island,

Vanuatr"

\section{INFORMED CONBENT FORM}

\begin{tabular}{|ll|l|}
\hline Researcher: & Joe MdCarter & School of Geography, Environment \\
Telephone: & 006443877783 & and Earth Sciences (SGEES) \\
Mobile & 0064274224209 & Victoria University of Wellington \\
Supervisor & Michael Gavin & PO BOx 600 \\
Tel. & 006444635195 & Wellington 6140 \\
Email: & & (04) $463-5337$ \\
\hline
\end{tabular}

Introduction

Victoria University of Wellington requires that all research involve Participants who are fully informed about my research, and consent to participate. The form that I'm about to read you has been designed in acoordance with these requirements. It is meant to ensure that research Participants and their communities are protected from any harm potentially arising from their participation in the reseanch process.

Purpose of the Study

This study, as part of my PhD research, is intended to fulfil the following objectives:

1. What are the perceived patterns of general TEK loss actoss Male kula?

2. How is disaster-related TEK changing actoss different sectors of the population in a focus community? This has two bey components:

a. How is diversity of disaster re lated plant knowledge changing?

b. How are traditional practices associated with disaster relief changing?

3. How are these changes impacting on local ability to respond and adapt to changing climatic conditions?

4. What are local perceptions of the best way to conserve disaster-related TEK?

Flease also see the 'Information Sheet'

Reserrch Format

I will undertake qualitati ve field research by camying out interviews, focus group discussions, and a quanti tative survey of traditional ecological knowledge with a variety of participants in your community. Participants will be asked to take part in three activities: a listingexercise, a walkthrough trail, and a short interview. Feople that wish may participate in a focus group towards the end of my stay. These activities are aiming to collect opinions about the key questions listed above. Participants are being asked to consent to the collection of theiropinions on traditional ecological knowledge. No names will be attached to any data collected and opinions will not be attributable to any one person. Each activity will last no more than one hour, and each focus group for no more than three houts. 
Please circle one

1. I understand that I am being asked to participate in series of activities, not totalling more than three houts.

$\mathrm{Y} / \mathrm{N}$

2. I understand that my name will never be revealed or attached to any data collected during this research project

$\mathrm{Y} / \mathrm{N}$

3. I understand that the data I provide will not be used for any other purpose than described in the 'Information Sheet' or rekased to others without written consent.

$$
\mathrm{Y} / \mathrm{N}
$$

4. I understand that data collected in this interview may be stored securely and accessed by Joe McCarter, Dr Michael Gavin and researchers approved by the National Cultural Council.

$$
\mathrm{Y} / \mathrm{N}
$$

5. I understand that a summary overview of the research program will be provided to my community at the completion of DiMichael Gavin's research program.

$$
\mathrm{Y} / \mathrm{N}
$$

6. I agree to take part in this research

$\mathrm{Y} / \mathrm{N}$

[Verbal consent. Should the participant not answer $Y$ to any of the above answers the interview etc will not be continued] 


\subsection{Semi-structured interview schedule (Chapter Seven)}

For this chapter there were two distinct interviews, the first for TEK experts and teachers on Malekula, and the second for officials in Port Vila. Please see the chapter for details.

\subsubsection{Community members and teachers}

1. Introduction, explanation of information and informed consent procedure

2. Verbal consent, questions

3. Do you think TEK is important in this community? Why?

4. Has TEK changed in this community? How? Why?

5. What is driving these changes in TEK?

6. What have been the effects of these changes within the community?

7. Who is currently teaching TEK in the community?

8. Whose job is it to teach TEK?

9. Can you teach TEK in formal school? Why or why not?

10. If TEK could be taught in school, which subjects do you think should be included?

11. What might be some of the problems with doing this?

\subsubsection{Officials in Port Vila}

12. Introduction, explanation of information and informed consent procedure

13. Written consent, questions

14. Do you think TEK is important in Vanuatu? Why?

15. Has TEK changed in Vanuatu? How? Why?

16. What is driving these changes in TEK?

17. Is TEK supported by formal school curricula at the moment? Why or why not? 
18. Is vernacular language supported by formal school curricula at the moment? Why or why not? What is the prognosis?

19. Do you think TEK could be integrated into formal school curricula or taught at schools? Why or why not?

20. What might be some of the barriers to teaching TEK at school?

a. Do you think these are surmountable?

21. What kinds of subjects do you think are appropriate for inclusion in formal schools?

a. How would TEK inclusion work in with current curriculum design?

22. Would trained teachers be able to teach TEK in school?

23. Do you think there is political support and institutional capacity for this kind of move?

24. Do you think this would have support at a community level? 


\subsection{Semi-structured interview schedule 2009-2010}

N.B: Where interviewees were also part of the sample for Chapter Eight (Appendix 11.8), the two interview schedules were combined $(n=10)$

\subsubsection{Introduction}

1. Introduction, explanation of information and informed consent procedure

2. Verbal consent, questions

\subsubsection{Freelisting (interview creation for Chapters Five and Six)}

3. Ethnomedical illness domains (Chapter Five)

a. Please tell me all the illnesses you treat using kastom medicine in this community

4. TEK transmission (Chapter Six)

a. Please tell me all the skills in the domain of building you think will be important in this community in the community in the future

b. Please tell me all the skills in the domain of food gathering and preparation you think will be important in this community in the community in the future

c. Please tell me all the skills in the domain of carving you think will be important in this community in the community in the future

d. Please tell me all the skills in the domain of weaving you think will be important in this community in the community in the future

\subsubsection{TEK transmission (qualitative data for Chapter six)}

5. Do you think TEK is important in this community? Why?

6. Has TEK changed in this community? How? Why?

7. How did people learn TEK before? Who was doing the teaching? Where was it conducted?

a. Was the same for everyone (i.e. boys and girls?) 
b. Was this the same for different domains of TEK (i.e. carving and weaving?)

8. How do people learn TEK now? Who does most of the teaching? Where is TEK teaching conducted?
a. Is the same for everyone (i.e. boys and girls?)
b. Is this the same for different domains of TEK (i.e. carving and weaving?)

9. What has changed about the ways that people here teach and learn TEK?
a. Why has this happened (drivers of change)?
b. What have been the effects of this? 


\subsection{Semi-structured interview schedule (Chapter Eight)}

N.B: Where interviewees were also part of TEK expert sample (Appendix 11.7) the two interview schedules were combined $(\mathrm{n}=10)$

10. Introduction, explanation of information and informed consent procedure

11. Verbal consent, questions

12. Do you think TEK is important in this community? Why?

13. Has TEK changed in this community? How? Why?

14. Please describe the kastom school in this community.

a. [If not already covered]:

b. Who will attend the kastom school, at what age??

c. What will be taught at the kastom school, and by who?

d. How will the school be managed?

e. Will the 'curriculum' be the same for boys and girls?

15. Why do you think the kastom school is needed here?

16. How will the kastom school be funded?

17. How did the kastom school come about?

18. What have been some of the problems you have faced?

a. Do you think these problems can be overcome? Why or why not?

19. Do the community support the kastom schools? Why or why not?

20. What is your prognosis for the schools going into the future?

21. What is your relationship with the kastom schools?

22. How long have you been involved? 


\subsection{Structured interview schedule (2009-2010)}

\subsubsection{Freelists}

1. Plant uses: Tell me all the uses you have for... [N.B: Data from this question not presented in this thesis]
a. Coconut
b. Pandanus
c. Naos
d. Wilken
e. Breadfruit
f. Black palm

2. Tell me all the different plant species you use to build houses here... [N.B: Data from this question not presented in this thesis]

3. Tell me all the different plant species you use for carving here... [N.B: Data from this question not presented in this thesis]

4. Tell me all the different plant species you use for weaving here... [N.B: Data from this question not presented in this thesis]

5. Tell me all the plant species you use in this community to treat these sicknesses
a. Headache
b. Cough
c. Earache
d. Diarrhoea
e. Toothache
f. Cuts
g. Arthritis
h. Malaria 
i. Hepatitis

j. Conjunctivitis

6. Tell me all the different kinds of wild food you can eat here if a hurricane ruins your garden... [N.B: Data from this question not presented in this thesis]

7. Tell me all the fish that live on the reef here...

8. Tell me all the different ways you know to catch fish, from today and before... [N.B: Data from this question not presented in this thesis]

\subsubsection{Skills transmission}

9. Skills transmission [For every skill, participants asked to self-rate proficiency on a 7point Likert scale, who they learnt the skill, the age of first skill transmission, and major location of skill transmission]

a. Pinning thatch

b. Cutting posts for houses using an axe

c. Tying thatch using vines from the bush

d. Weaving bamboo

e. Make a 'laplap wota'

f. Catching freshwater crayfish using hands

g. Herbal medicine to make large yams

h. Poison for catching fish

i. Herbal medicine

j. Carving tamtam

k. Carving nalnal

1. Making a bow and arrow

m. Carving canoes 

n. Weaving a grass skirt
o. Weaving a mat of pandanus
p. Weaving a basket of pandanus
q. Weaving a fan
r. Weaving a rope of coconut fibre

\subsubsection{Ecological knowledge}

10. Plants (photos by author)
a. Naisete (scientific name unknown)
b. Pueraria lobata
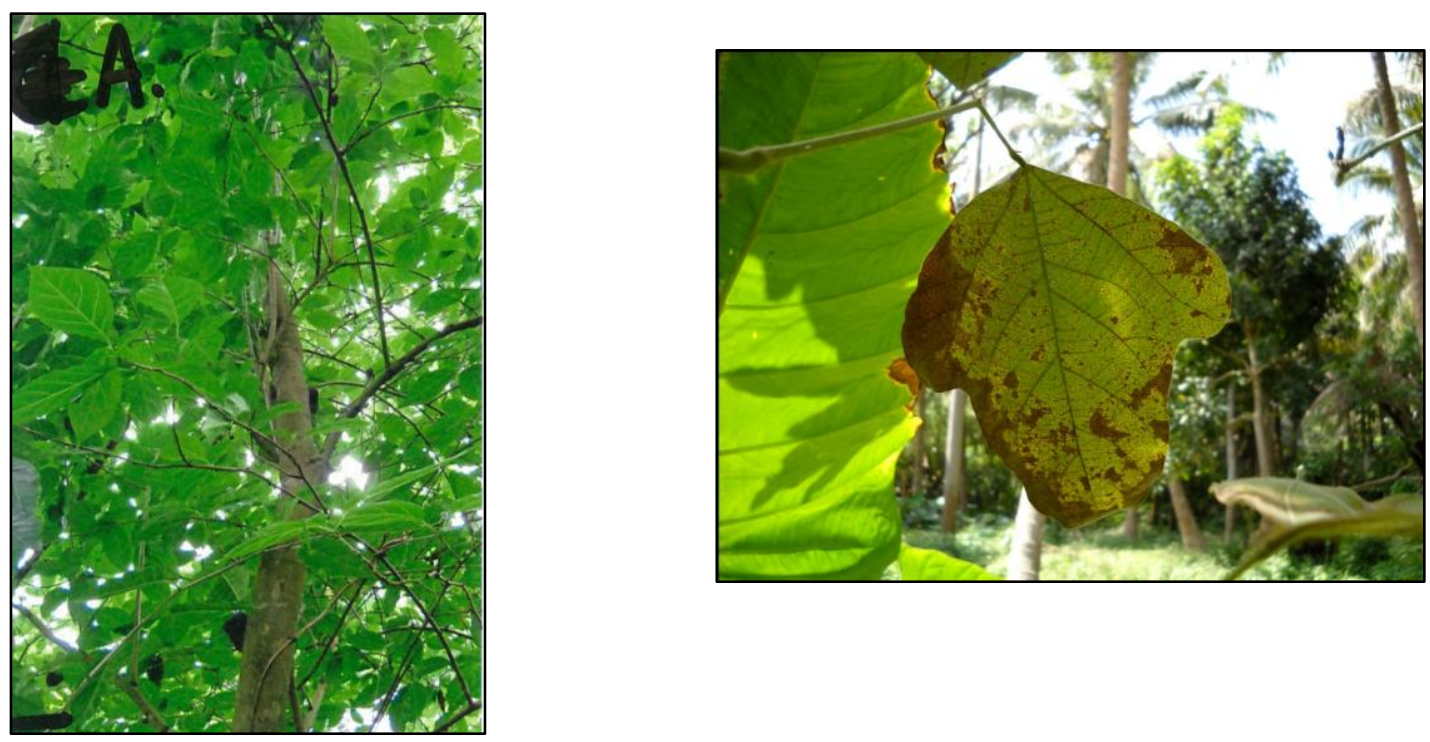

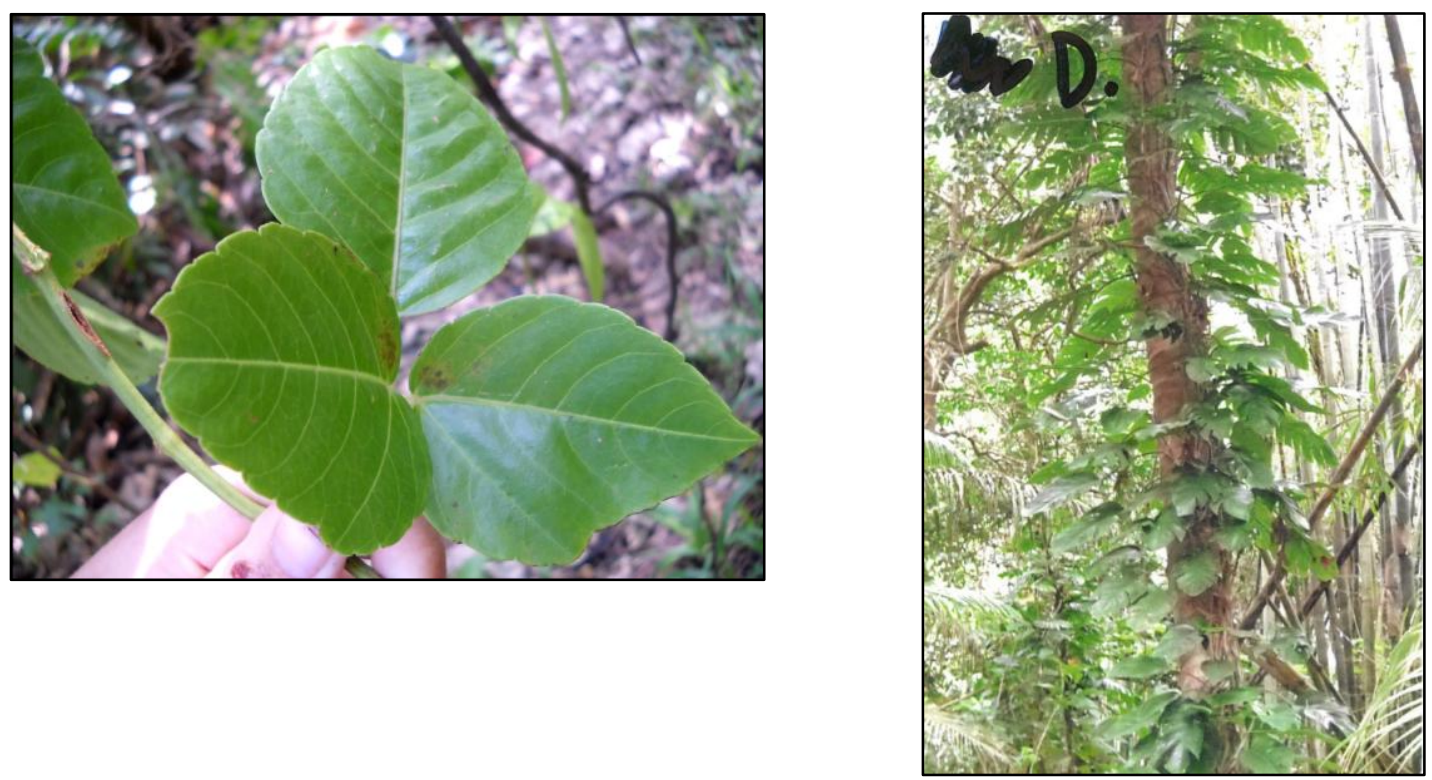

e. Acaphyla grandis

f. Macaranga divoica
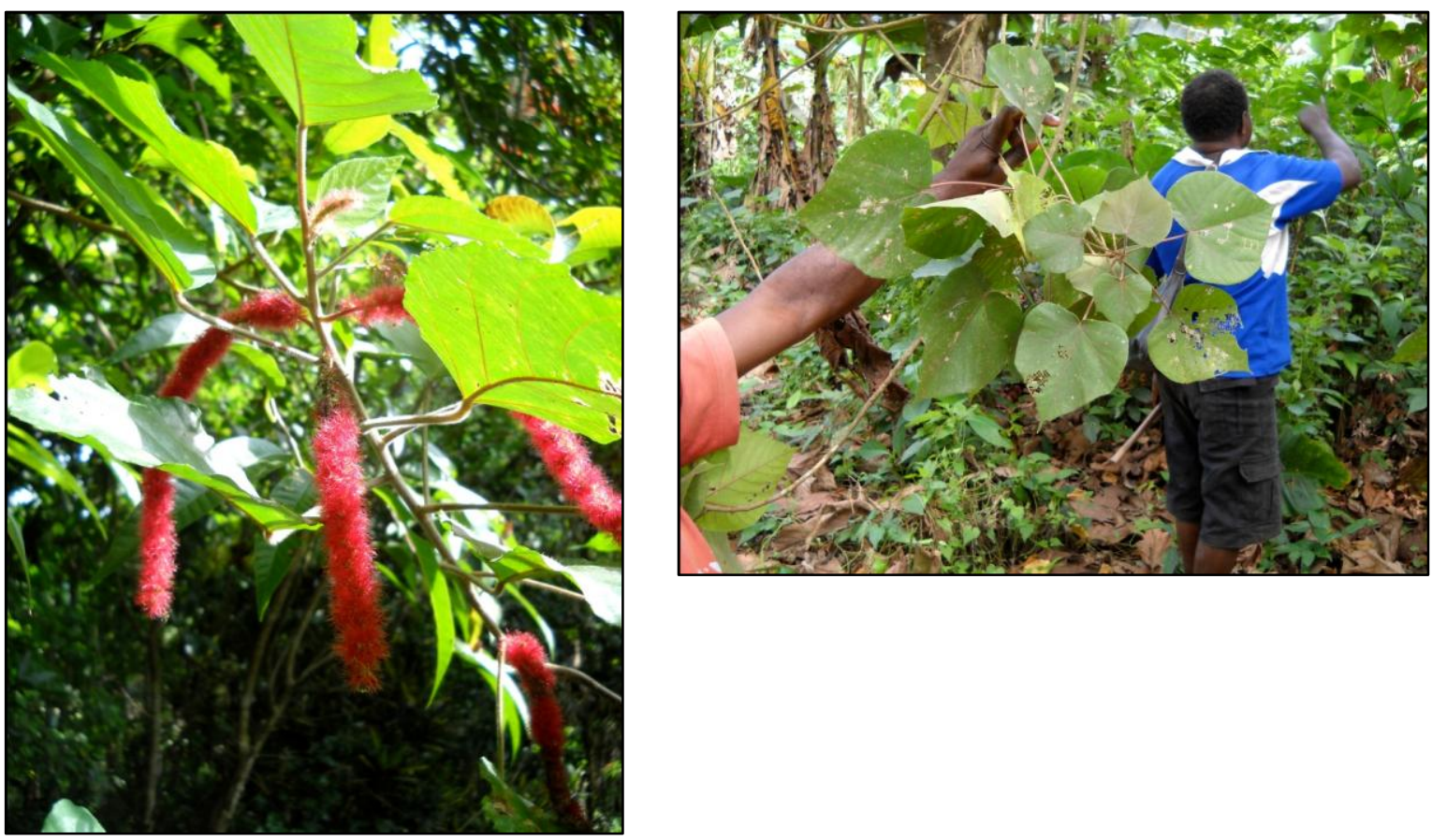
11. Birds (photos from Bregulla 1992)

a.Silver eared honeycatcher

b. Pacific imperial pigeon
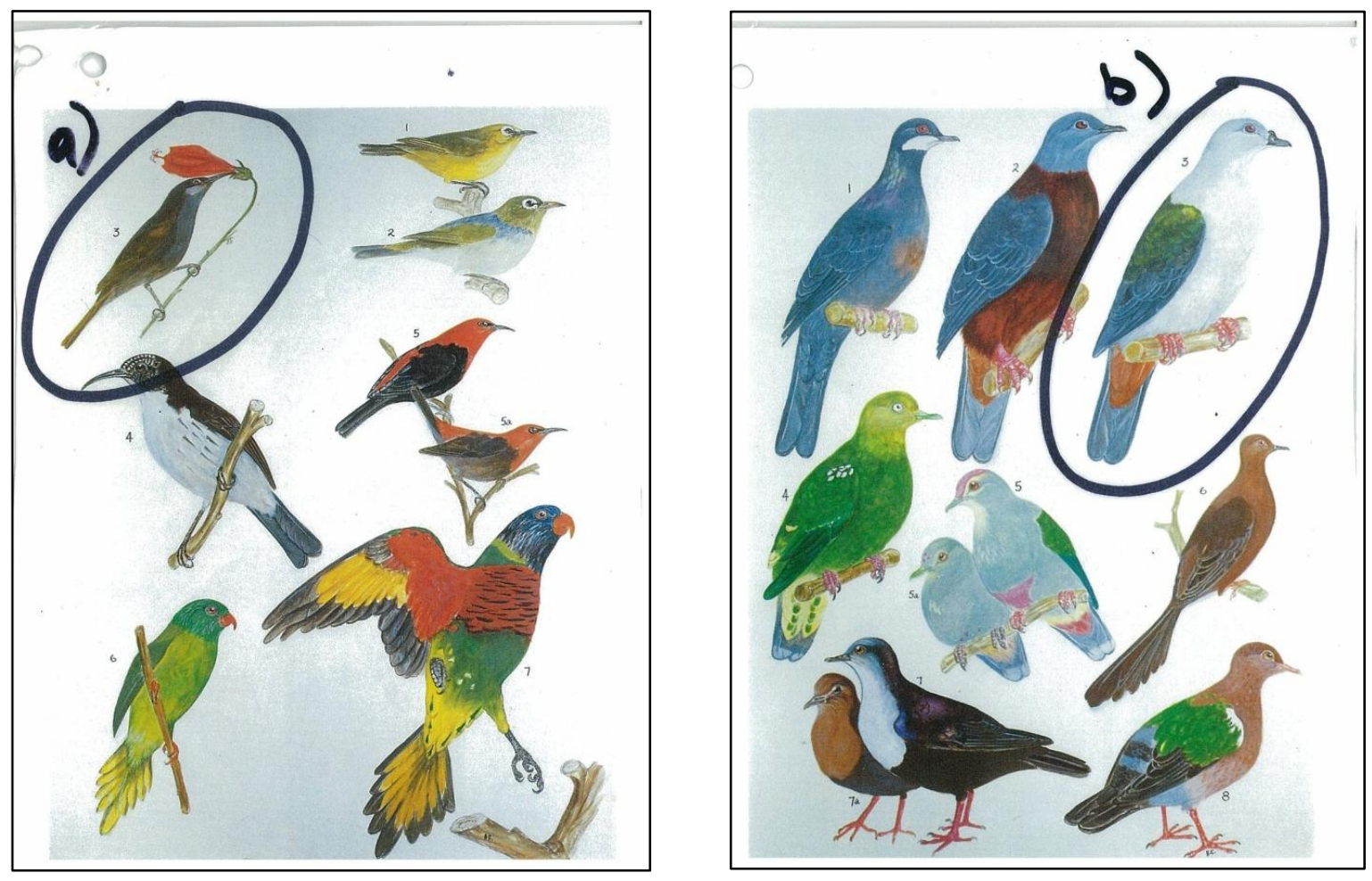
c.Spotted fantail

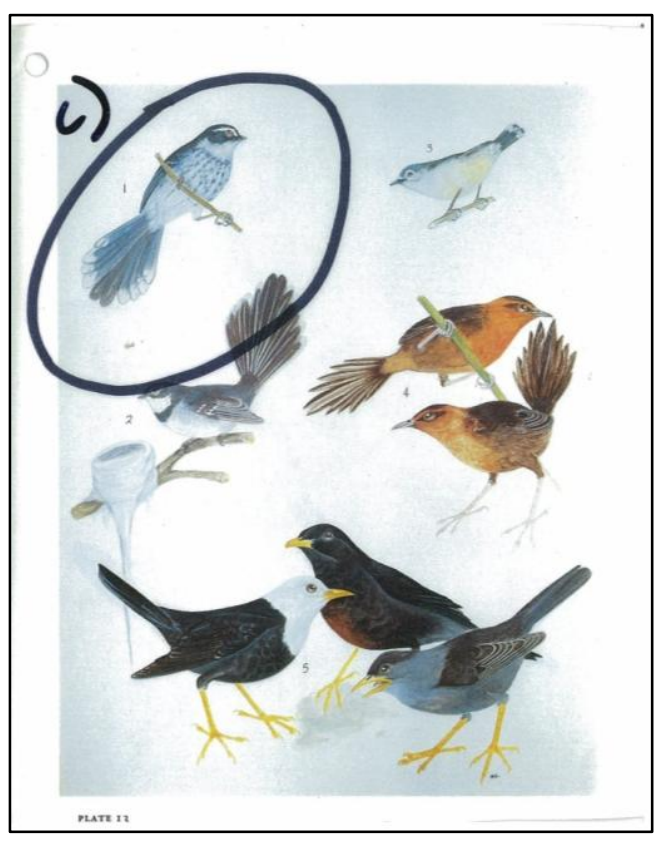

12. Shellfish (photos from Allen and Steene 1994)
a. Topshell
b. Nerite

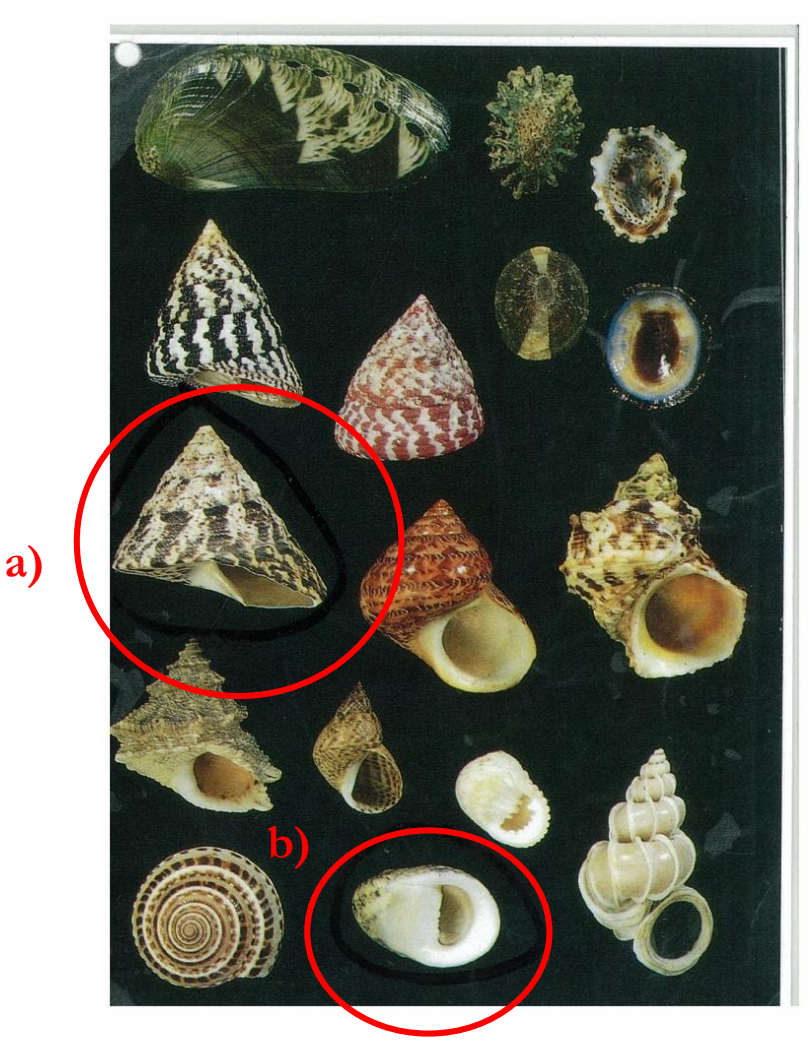


13. Fish (photos from Lieske and Myers 1999)

a. Reef needlefish

b. White spot soldierfish

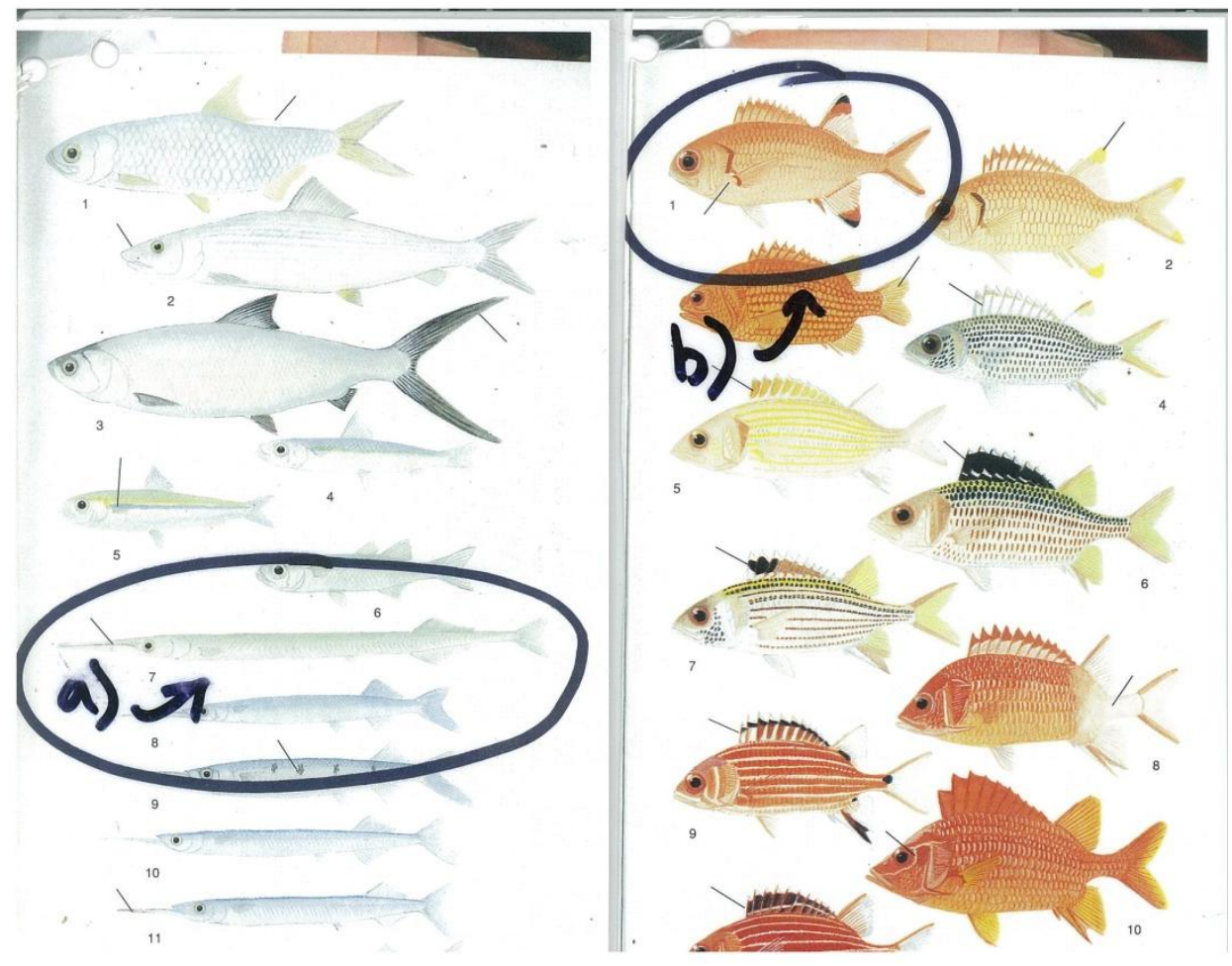


c. Black fin barracuda

d. Violet lipped parrotfish
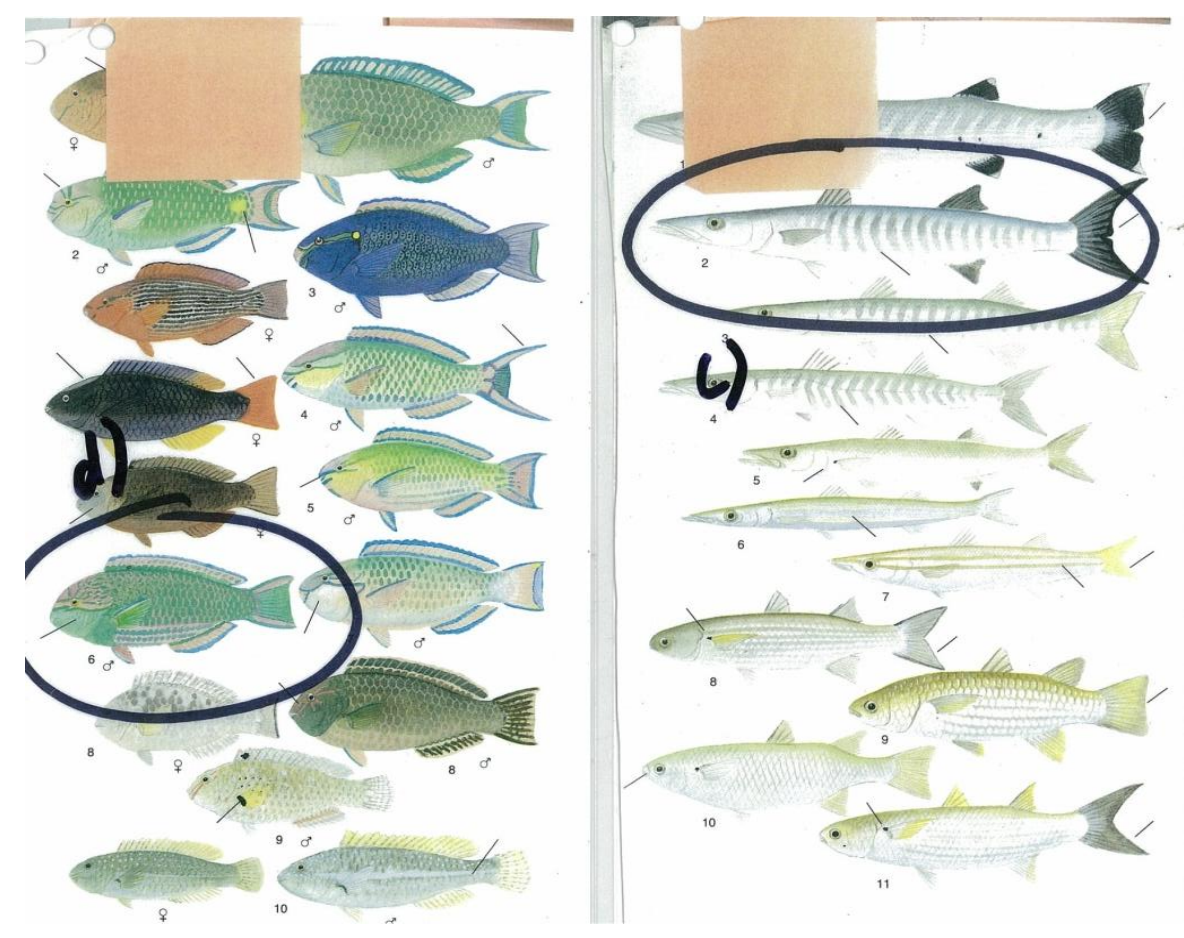

e. Racoon butterflyfish

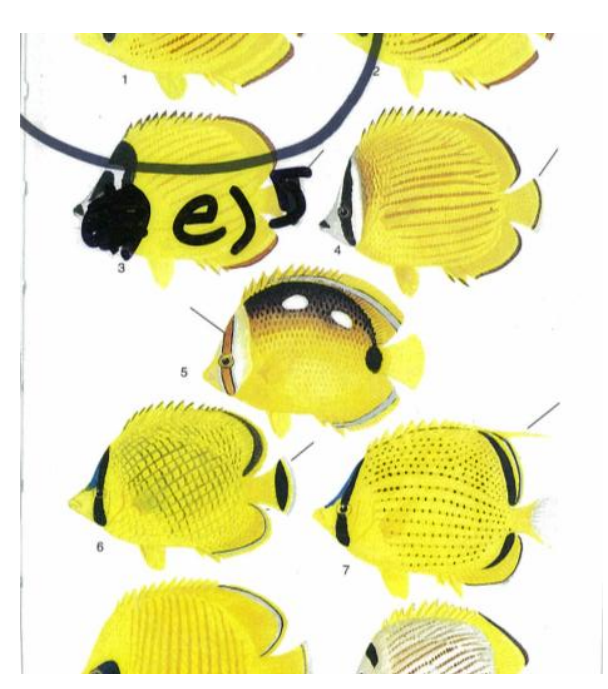

14. Ecology

a. What is the favourite food of the Pacific swallow: insects, fruit, or flower? 
b. The navenou tree flowers during which period: Nov-Jan, Feb-Mar or JunAug?

c. Are female black flying foxes larger than males?

d. What is the favourite food of the green sea turtle: coral, fish, or seagrass?

e. Do black crabs lay their eggs before white crabs every year?

\subsubsection{Socio-demographic information}

15. Gender

16. Age

17. Which year did you reach at school?

18. How many languages do you speak?

19. Which language do you prefer to use every day?

20. Which church do you belong to?

21. How many times have you been to church in the last four weeks?

22. Where were you born?

23. How long have you lived here?

24. When did your family arrive here?

25. How many times have you been to Lakatoro, Port Vila, or Luganville in the past year? 


\title{
11.10 Scoping study structured interview schedule
}

\author{
Interview Schedule - Structured
}

1. Do you think TEK is important now? Why?

2. Trend analysis

2.1. Please tell me about the change between these two places [indicate to t.a]

2.2. What is causing these changes?

2.3. Do people know the same things between here and here?

2.4. Do people use the same things between here and here?

2.5. Do people use tabu the same between here and here?

2.6. Do people respect tabu as much between here and here?

[Repeat 3 times]

3. Discussion of change

3.1. When your grand parents were children, how much TEK did their community have?

3.2. How did they manage and protect their environment?

3.3. When your parents were children, did their community have the same amount of TEK?

3.4. Did they know as many things?

3.5. Did they use as many things?

3.6. Did they respect tabu he same?

[Repeat for 'when you were a clild' and 'now']

4. Types of change [using a 5 point scale: No Change, Small Change, Medium Change, Big Change, and Gone]

4.1. Knowledge of different plant species

4.2. Knowledge of different fish species

4.3. Knowledge of plants that can be used for medicine

4.4. Fashion that you build houses

4.5. Fashion that you build canoes

4.6. Fashion that you work in the garden

4.7. Fashion that you make and place tabu

4.8. Fashion that tabu are respected

4.9. Fashion that the community here protects the environment

4.10. Fashion that people believe in the devils of the bush

5. How have the changes that you have been telling me about affected this place?

6. Teaching TEK

6.1. Are children learning enough TEK?

6.2. Where are the learning TEK?

6.3. Who is teaching TEK?

6.4. Where should children learn TEK? 
6.5. Whose job is it to teach TEK?

6.6. Does school teach TEK?

6.7. Should school teach TEK?

6.8. Does the community here make enough work teaching TEK?

6.9. Does school make children disrespect tabu?

6.10. What language should you teach TEK in?

7. Aspects of change [using a 7 point scale: Strongly Agree, Agree, Partially Agree, Don't Know/Don't Care, Partially Disagree, Disagree, Strongly Disagree

7.1. School helps children with TEK

7.2. School should teach TEK

7.3. Teachers at school cannot teach TEK

7.4. The chiefs here should be making more work teaching TEK

7.5. The community here helps with teaching TEK

7.6. Tourists are damaging TEK

7.7. Foreign goods [inadequate translation - 'ol samting blong waetman'] are damaging TEK

7.8. We can teach TEK in Bislama

7.9. We can teach TEK in English

7.10. Life in Port Vila is not good for TEK [leading, I think?]

7.11. The church(es) here are damaging TEK

7.12. School makes children respect tabu more

7.13. School can teach TEK and science together

7.14. Population growth makes TEK go down [leading, probably discard]

7.15. When the missionaries came they damaged TEK

8. Future

8.1. Trend analysis/further discussion

8.1.1. [if section 1 before] What do you think will change in the future? Why?

8.1.2. [if section 2 before] If we look $20 y$ into the future, how much

TEK do you think this community will have? Why?

8.2. What can we do to preserve and nurture TEK, for the children today and future generations?

8.3. Is TEK preservation work for the community or government? If government, what do you think it can do to help [as a discussion, good closing question, more than for future analysis]

9. Collection of age, birthplace, and level of schooling. 


\subsection{Scoping study methods}

\subsubsection{Sample}

In total 120 participants were interviewed in the five communities described in Chapter Four (Table 11.1). Participants were selected using snowball sampling (Bernard 1998). Participant selection was roughly stratified by age (when broken into three rough cohorts of 18-30; 31-60; and 61+) and gender, because TEK has been shown to be patterned by demographic variables such as these (Boster 1986; Phillips and Gentry 1993). However, it was not possible to get an even spread of participants in each community, and most notably there was a clear gender bias present in three of the five communities. This was a product of two factors: first, there is a bias inherent in snowball sampling, as one is dependent on the social networks of the original informants (Bernard 1998). As my primary informants were all men, they tended to recommend men as interview targets. Second, women were less comfortable with the interview process, and often refused to be interviewed.

Table 11.1: Participants in structured interview (Phase one)

\begin{tabular}{llccccc}
\hline Area & Community & $n$ & Male & Female & Median age & Range \\
\hline \multirow{2}{*}{ Urupiv } & Jimes & 24 & 12 & 12 & 57.5 & $20-80$ \\
\cline { 2 - 8 } & Tevri & 21 & 15 & 6 & 42 & $18-80$ \\
\hline \multirow{2}{*}{ SW Bay } & Lorlow & 26 & 16 & 10 & 48.5 & $18-80$ \\
\cline { 2 - 8 } & Verengeboas & 8 & 5 & 3 & 27.5 & $20-50$ \\
\hline \multirow{2}{*}{ Unmet } & Unmet & 41 & 26 & 15 & 48 & $22-90$ \\
\hline \multirow{2}{*}{ Total } & & $\mathbf{1 2 0}$ & $\mathbf{7 4}$ & $\mathbf{4 6}$ & $\mathbf{4 8}$ & $\mathbf{1 8 - 9 0}$ \\
\hline
\end{tabular}

This research was conducted under strictly ethical conditions according to according to the procedure outlined in Section 3.3.2. All participants were adult, fully informed, gave their verbal consent to the interview and publication process. All participants remain confidential.

\subsubsection{Field methods}

Data were gathered using structured interviews (Bernard 1998). This method is useful for gathering rigorous, repeatable and comparable data for use in intra- or inter-cultural comparisons. Structured interviews are also more suitable for maximising sample size, and so increasing the robustness of the findings. The interview covered a range of topics (included in Appendix 11.10) The first section discussed the nature of recent changes on TEK and asked about the primary drivers of these changes. I used two techniques to elucidate the timing of those changes, and specify which domains of knowledge had changed. 
To assess the timing and magnitude of changes in TEK (as well as participant projections into the future) I used trend analysis, a participatory technique described in Kumar (2002). Participants were asked to represent the level of TEK in their community across time by placing a certain number of units (here, I used dried beans) on five panels representing different time periods, from 'when your grandparents were children' to '20 years in the future' (Figure 11.1). This was conducted in 57 of the interviews in total: 43 on Urupiv, 7 in SW Bay, and 7 at Unmet. I 'anchored' the trend analysis (and so provided a reference point) by placing a set number of units on one of the panels. I used two methods here to check the validity of the results: for 29 of the interviews I placed 30 units on the panel describing when their grandparents were children, and for the other 28, I placed 10 units on the panel representing the present time.

Trend analysis provides an estimate of change across time, although it is clearly not an exact measure of diachronic changes in TEK in the community. Participants were of different ages, so were referring to different time periods, and may recall changes imperfectly (recall bias). However, the technique is a useful indicator of participant perceptions on the subject, and provides a focus for discussion of the drivers of TEK erosion (Kumar 2002).

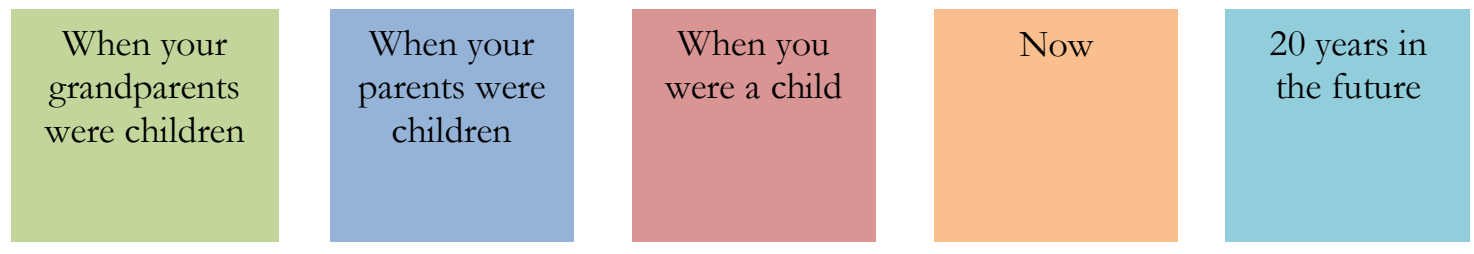

Figure 11.1: Layout of trend analysis panels

Next, to assess how TEK had changed across different domains and dimensions I asked participants to describe the degree of change in ten domains of TEK between the time when their grandparents were children and the present time. ${ }^{27}$ Participants were asked to rate the degree of change in each domain of knowledge on a five-point Likert scale. The points on the scale were named with the degree of change they represented: 'no change', 'small change', 'medium change', 'large change', and 'gone already'. ${ }^{28}$

The ten sampled domains were based on the heuristic description of TEK in Berkes (2008), which proposes that TEK be thought of as a complex comprising various dimensions, including knowledge, practice, and belief (Section 2.1). Questions were based on the literature and were checked with local representatives of the VCC and Malekula Cultural Centres. The 'knowledge' domains of the survey

\footnotetext{
${ }^{27}$ In Bislama: "Yu stap ting se [dimension of TEK] hemi bin jenis hamas [indicate to Likert scale] afta taem we ol bubu blong yu oli pikinini nomo..."

${ }^{28}$ In Bislama: i no gat jenis; smol jenis; medel jenis; bigfala jenis; gone finis
} 
asked participants to describe levels of theoretical knowledge of the surrounding flora and fauna. The 'practice' aspects were based around various practical skills that were likely to be still widely used in the community, especially the use of $t a b u$ (in this context, spatial and temporal restrictions on resource use (e.g. Hickey 2005). The belief dimension of TEK was represented by one question, which referred to the 'devils' of the bush. In this context, this referred to spirits which formed a critical part of pre-Christian spirituality of the island, and were especially associated with respect of tabu and the maintenance of social norms (Hickey 2005). The belief dimensions in knowledge are important in representing the worldview within which TEK is contained, and are often neglected from measures of TEK due to difficulty of measurement.

Table 11.2: Domains of knowledge for inclusion in structured interview

\begin{tabular}{cl}
\hline Domain & Dimension of TEK \\
\hline \multirow{3}{*}{ Knowledge } & Knowledge of all local plants \\
& Knowledge of local fish \\
& Knowledge of medicinal local plants \\
\hline & Style of house construction \\
& Style of canoe construction \\
& Style of creating garden \\
& Style of placing tabu \\
& Style of respecting tabu \\
& Style of protecting natural resources \\
\hline Belief & Belief in 'devils' of the bush
\end{tabular}

All of the interviews in Phase one were undertaken with the assistance of a translator, although my ability to conduct interviews independently in Bislama improved over the course of the research program. The use of a translator is not without its difficulties (Scheyvens and Storey 2003), and this initial scoping research was not immune to this concern. Whilst the data gleaned during this time do not appear to differ significantly from the remainder of the interviews, it would be appropriate to treat the results of this scoping study with some caution.

\subsubsection{Analysis}

Data were entered into SPSS 17.0 (SPSS Inc. 2008) and analysed using basic non-parametric statistics including Kruskal-Wallis (between multiple categories), Mann-Whitney U (between two categories), and Friedman's non-parametric ANOVA where appropriate (Field 2009). 


\subsection{Vascular plant families cited during freelisting}

\begin{tabular}{|c|c|c|c|c|c|c|c|}
\hline Family & $\begin{array}{c}\text { Number } \\
\text { of } \\
\text { genera }\end{array}$ & $\begin{array}{c}\text { Number } \\
\text { of } \\
\text { species }\end{array}$ & $\begin{array}{c}\text { Number } \\
\text { of } \\
\text { citations }\end{array}$ & Family & $\begin{array}{c}\text { Number } \\
\text { of } \\
\text { genera }\end{array}$ & $\begin{array}{c}\text { Number } \\
\text { of } \\
\text { species }\end{array}$ & $\begin{array}{c}\text { Number } \\
\text { of } \\
\text { citations }\end{array}$ \\
\hline Acathanaceae & 3 & 3 & 4 & Lauraceae & 3 & 3 & 2 \\
\hline Agaveaceae & 1 & 1 & 6 & Laxmanniaceae & 1 & 2 & 3 \\
\hline Alliaceae & 1 & 1 & 4 & Lecythidaceae & 1 & 3 & 30 \\
\hline Amaranthaceae & 1 & 1 & 5 & Lycopodiaceae & 1 & 1 & 3 \\
\hline Amarylldaceae & 1 & 1 & 3 & Lythraceae & 1 & 1 & 1 \\
\hline Anacardiaceae & 4 & 5 & 28 & Malvaceae & 10 & 13 & 110 \\
\hline Annonaceae & 2 & 2 & 9 & Marantaceae & 1 & 1 & 9 \\
\hline Apocynaceae & 6 & 7 & 27 & Marattiaceae & 1 & 1 & 3 \\
\hline Araceae & 2 & 4 & 64 & Meliaceae & 4 & 6 & 6 \\
\hline Araliaceae & 2 & 3 & 26 & Menispermaceae & 1 & 1 & 1 \\
\hline Arecaceae & 3 & 3 & 130 & Moraceae & 4 & 11 & 109 \\
\hline Asphodelaceae & 1 & 1 & 4 & Musaceae & 1 & 1 & 29 \\
\hline Aspleniaceae & 1 & 1 & 1 & Myristicaceae & 1 & 2 & 10 \\
\hline Asteraceae & 4 & 4 & 197 & Myrtaceae & 3 & 5 & 60 \\
\hline Bixaceae & 1 & 1 & 1 & Nyctaginaceae & 1 & 2 & 17 \\
\hline Boraginaceae & 2 & 5 & 46 & Oleaceae & 1 & 1 & 1 \\
\hline Burseraceae & 2 & 2 & 7 & Orchidaceae & 1 & 1 & 2 \\
\hline Cannabaceae & 3 & 3 & 2 & Pandanaceae & 1 & 2 & 10 \\
\hline Caricaceae & 1 & 1 & 51 & Phyllanthaceae & 6 & 7 & 43 \\
\hline Casuarinaceae & 1 & 1 & 1 & Phytolaccaceae & 1 & 1 & 5 \\
\hline Clusiaceae & 1 & 1 & 3 & Piperaceae & 1 & 3 & 50 \\
\hline Combretaceae & 1 & 3 & 31 & Poaceae & 10 & 10 & 70 \\
\hline Commelinaceae & 1 & 1 & 3 & Portulacaceae & 1 & 1 & 1 \\
\hline Connaraceae & 1 & 1 & 1 & Pteridaceae & 1 & 2 & 2 \\
\hline Convolvulaceae & 3 & 8 & 47 & Rhamnaceae & 2 & 2 & 5 \\
\hline Costaceae & 1 & 1 & 4 & Rubiaceae & 3 & 3 & 11 \\
\hline Cucurbitaceae & 3 & 3 & 24 & Rutaceae & 3 & 5 & 56 \\
\hline Cyatheaceae & 1 & 2 & 6 & Sapindaceae & 3 & 3 & 39 \\
\hline Cycadaceae & 1 & 1 & 10 & Sapotaceae & 4 & 5 & 2 \\
\hline Davailliaceae & 1 & 1 & 1 & Solanaceae & 3 & 3 & 55 \\
\hline Dioscoreaceae & 1 & 3 & 13 & Tectariaceae & 1 & 2 & 4 \\
\hline Ebenaceae & 1 & 1 & 1 & Urticaceae & 4 & 4 & 34 \\
\hline Euphorbiaceae & 12 & 15 & 126 & Vitaceae & 2 & 2 & 16 \\
\hline Fabaceae & 17 & 20 & 86 & Vittariaceae & 1 & 1 & 1 \\
\hline Goodeniaceae & 1 & 1 & 1 & Zingiberaceae & 3 & 6 & 33 \\
\hline Hernandiaceae & 1 & 1 & 7 & Total & 73 & 237 & 1845 \\
\hline Heliconiaceae & 1 & 1 & 10 & $\begin{array}{l}\text { Unidentified folk } \\
\text { species }\end{array}$ & & 26 & 129 \\
\hline Lamiaceae & 4 & 6 & 22 & Total & 73 & 263 & 1974 \\
\hline
\end{tabular}




\subsection{Resource book (Dixon Reef)}

This is one of four resource books made for communities for use in the kastom schools and other ethnobotanical teaching. Several hundred copies have been sent to the focus communities as one of the key outputs of this work. The books were produced in Bislama only.

\section{Page One}

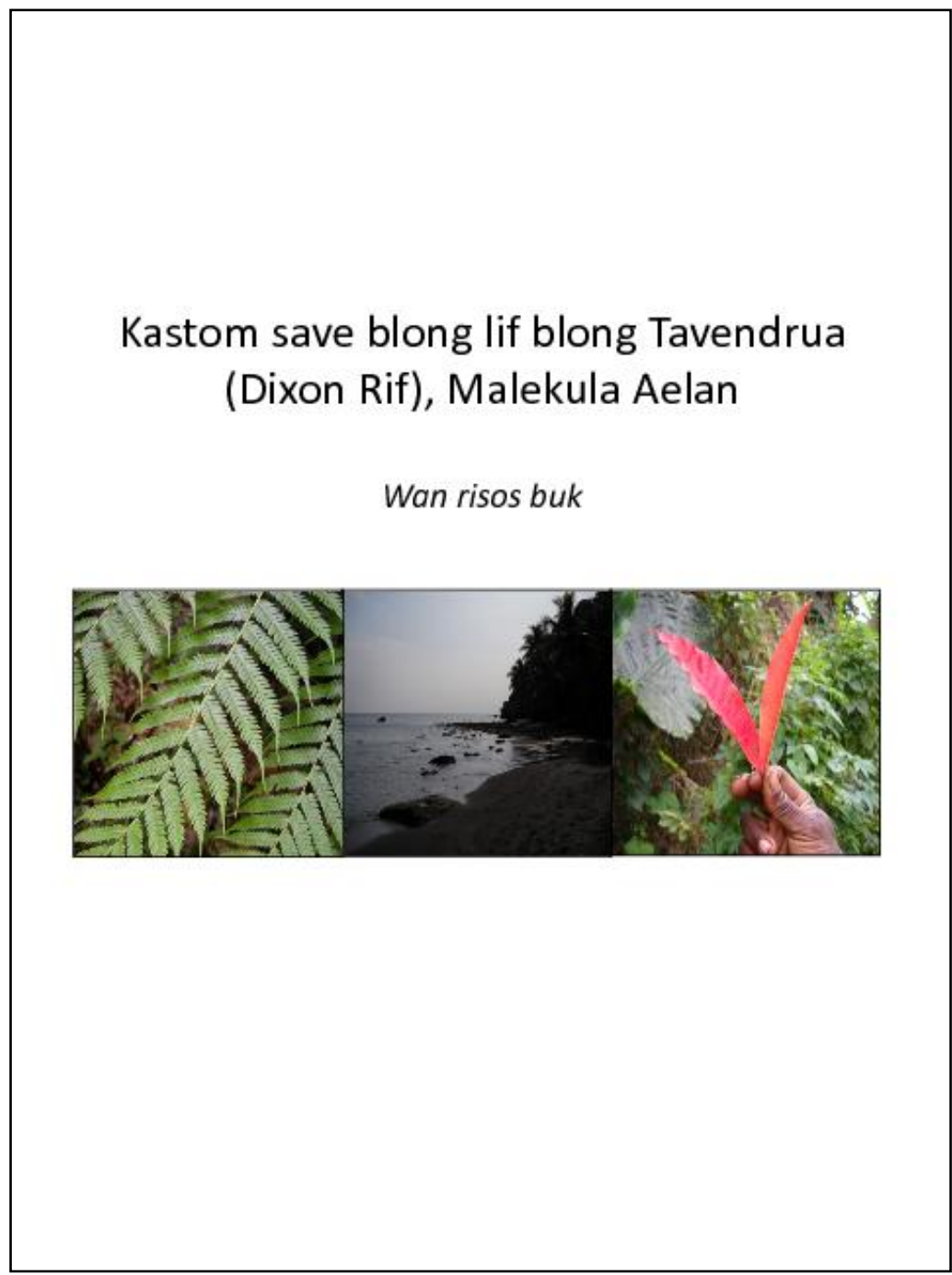


Pages Two and Three: information

Wanem ia kastom save blong envaeromen? Kasto $m$ save blong envaeromen I stap minim fulap samting:

- Ol save we ol bubu blong bifo I bin gat saed long ol lif, ol pijen, ol

fis, ol bebet, mo nara samting we I laef long graon mo wota

- Ol save we ol bubu blong bifo oli bin gat saed long hao nao blong lukaotem ol samting ia

- ol save blong bifo saed long ridim ol cloud mo weathe

- Ol bilif blong bifo we I b in lukaotem envaeromen

From wanem yumi nidim kastom save blong envaeromen? Kasto $m$ save blong envaeromen hemi important tumas long laef blong yumi. Ol save we ol oldfala I bin gat hemi save givhan long yu mi tedei blong stretem evri kaen problem wetem envaeromen-olsem mekem se yumi no spoilem envaeromen tumas. Mo too, I save givhan blong mekem se kastom mo kalja hemi strong long vilaj blong yumi. ol bubu oli bin waes tumas, mo bae yumi no wantem lusum waes ia.

\section{Kastom save I stap lus}

Taem blong ol bubu evri man I bin lanem ol kastom save blong envaeromen. Be naoia, fulap long ol oldfela I ded fin is, mo ol man mo woman tedei oli no lanem ol save blong bifo. Yumi mas lukaot se bambae ol kastom save blong envaeromen I no lus long fiuja.

\section{Wanem bukia?}

Buk ia bae I traem givhan blong mekem se kastom save blong ol lif I no lus. Ol waes blong bukia I kam long ol man mo woman long Tavend rua. Joe McCarter blong Victoria University (long Niu Zeal and), hemi bin raetem ol samting ia daon, mo Kaljoral Senta blong Vanuatu hemi bin givhan fulap.

\section{Risej blong Joe McCarter}

Risejia I stap traem faedema ot hao noa kastom save blone envaeromen I stap lus, mo hao nao yumi save leftemap ol save ia bakagen. Hemi bin startem work long 2008, mo hemi stap work long 4 ples long Malekula Aelan. Risos buk ia I kam aot long risej program ia.
Wanem insaed bukia?

Buk ia I gat sam smol infomesen saed long ol lif we ol man mo

woman long Lawa oli stap yusum. Bae vu go luk long buk ia mo traem luksave evri foto long ol lif mi evri nem we I stap long ol list. sipos yu no save, bae yu save go askem wan man o woman we oli save.

Insaed buk ia I gat:

Pej 4-7: Sam foto blong luk sipos yu luksave o no

Pej 8-11: Sam list long evri lif we man I telem long me long intervit

Pej 12: Sam waes we man I bin telem long saed lone tijim bakagen kastom save

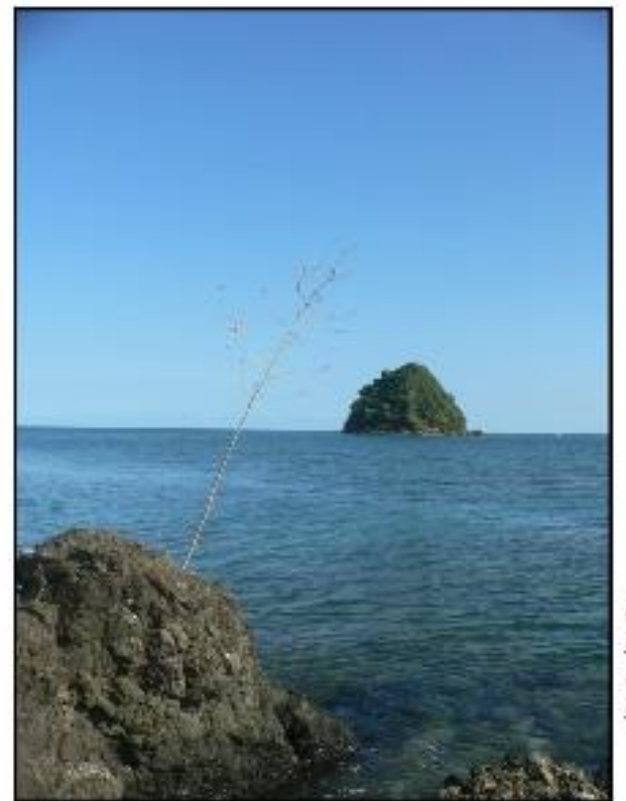

astom fasin blong bloken

solvota oli important tumas lon

Malekula. Hemio part long kastom save blong envoeromen. 
Pages Four and Five: photo identification

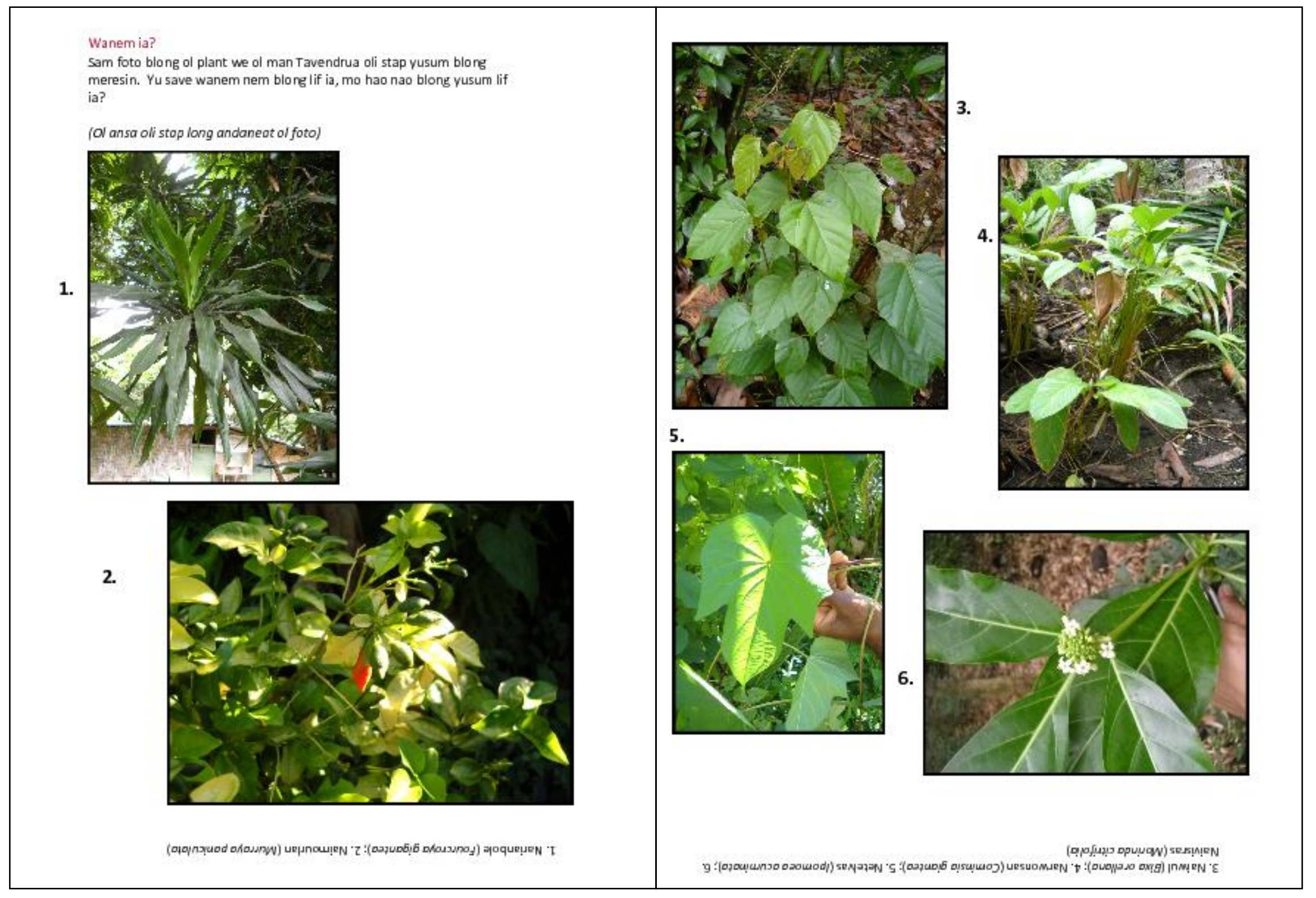


Pages Six and Seven : Photo identification

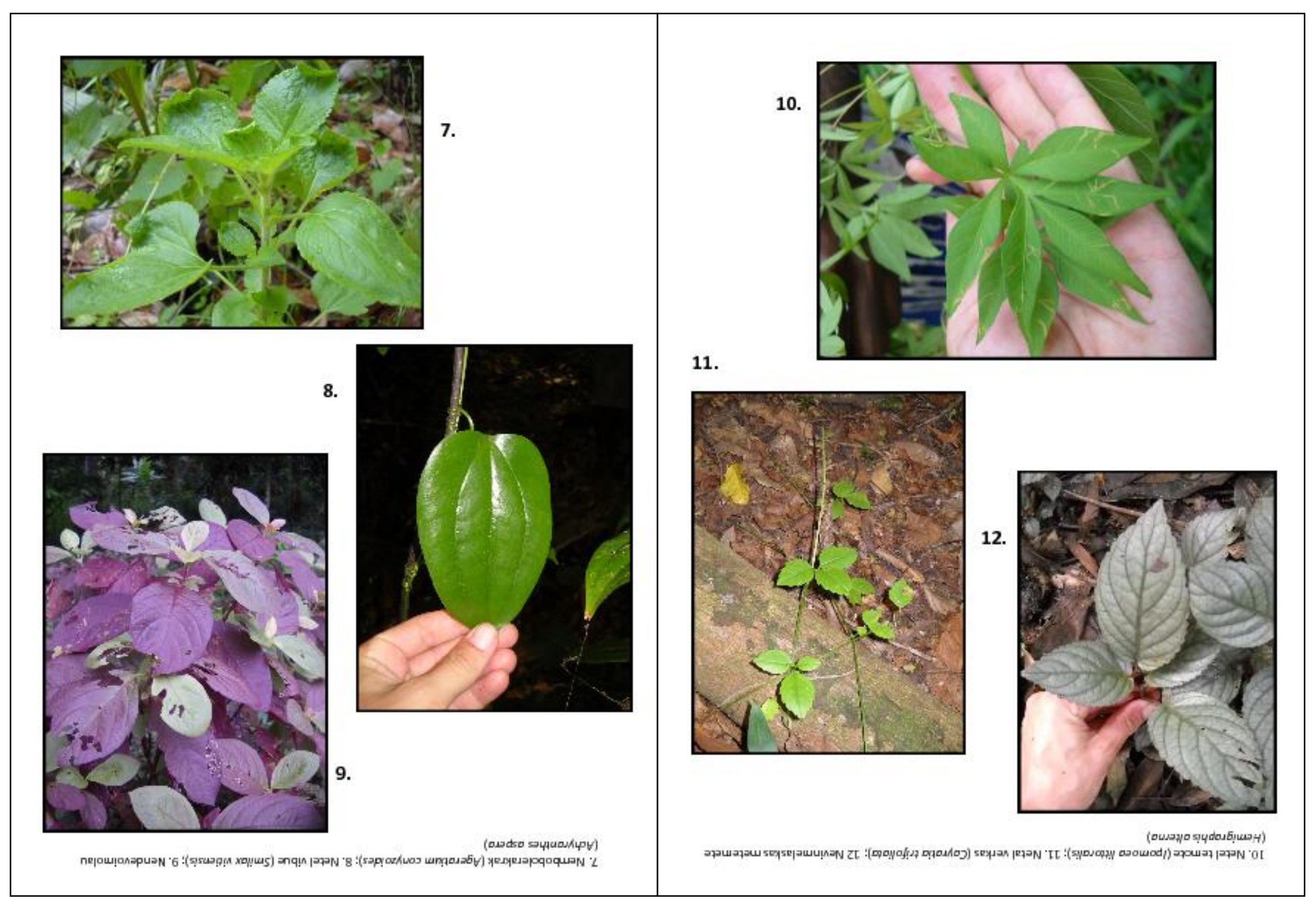


Pages Eight and Nine: Medicinal species checklists

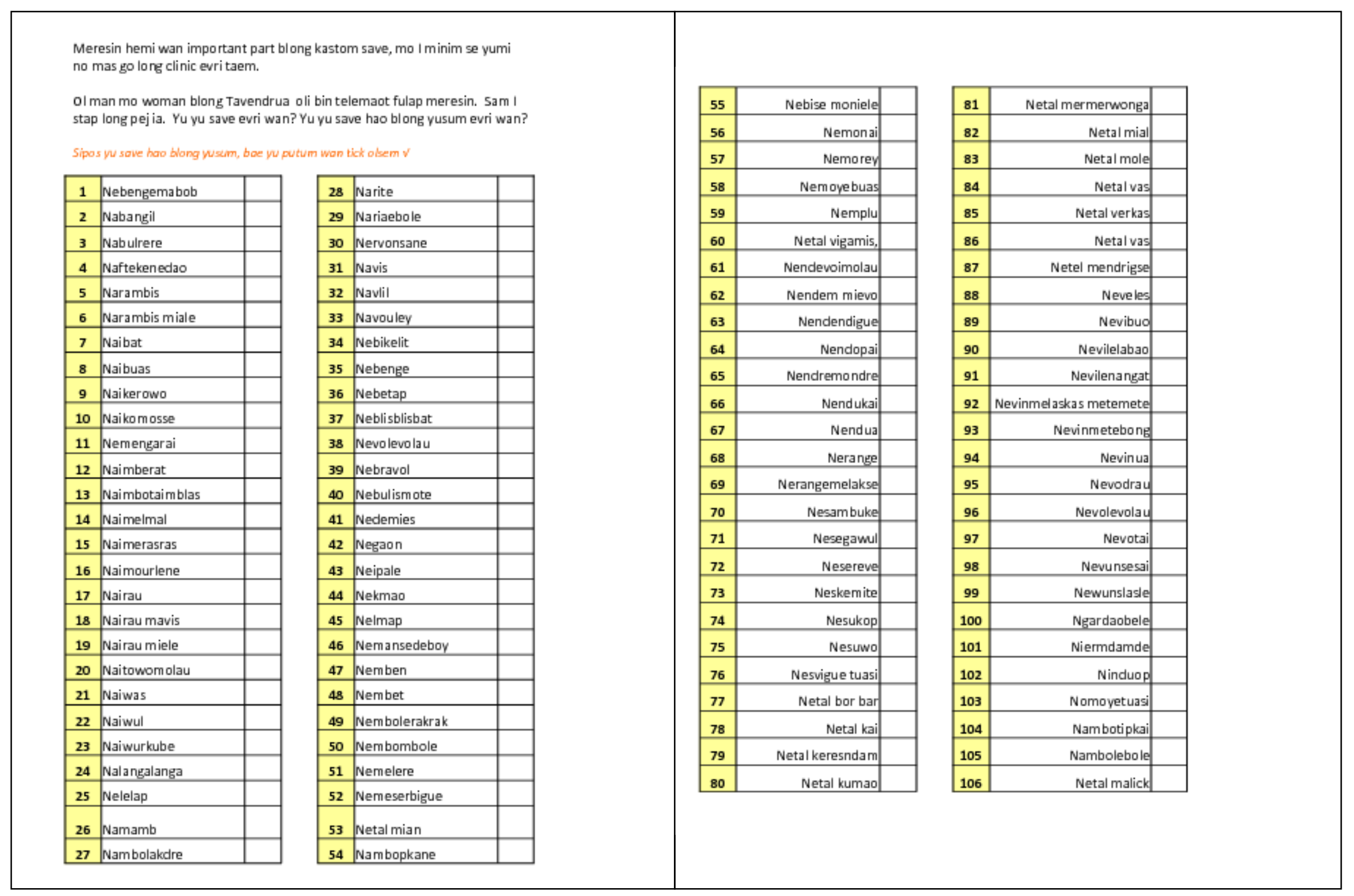


Pages Ten and Eleven: Other useful species checklist

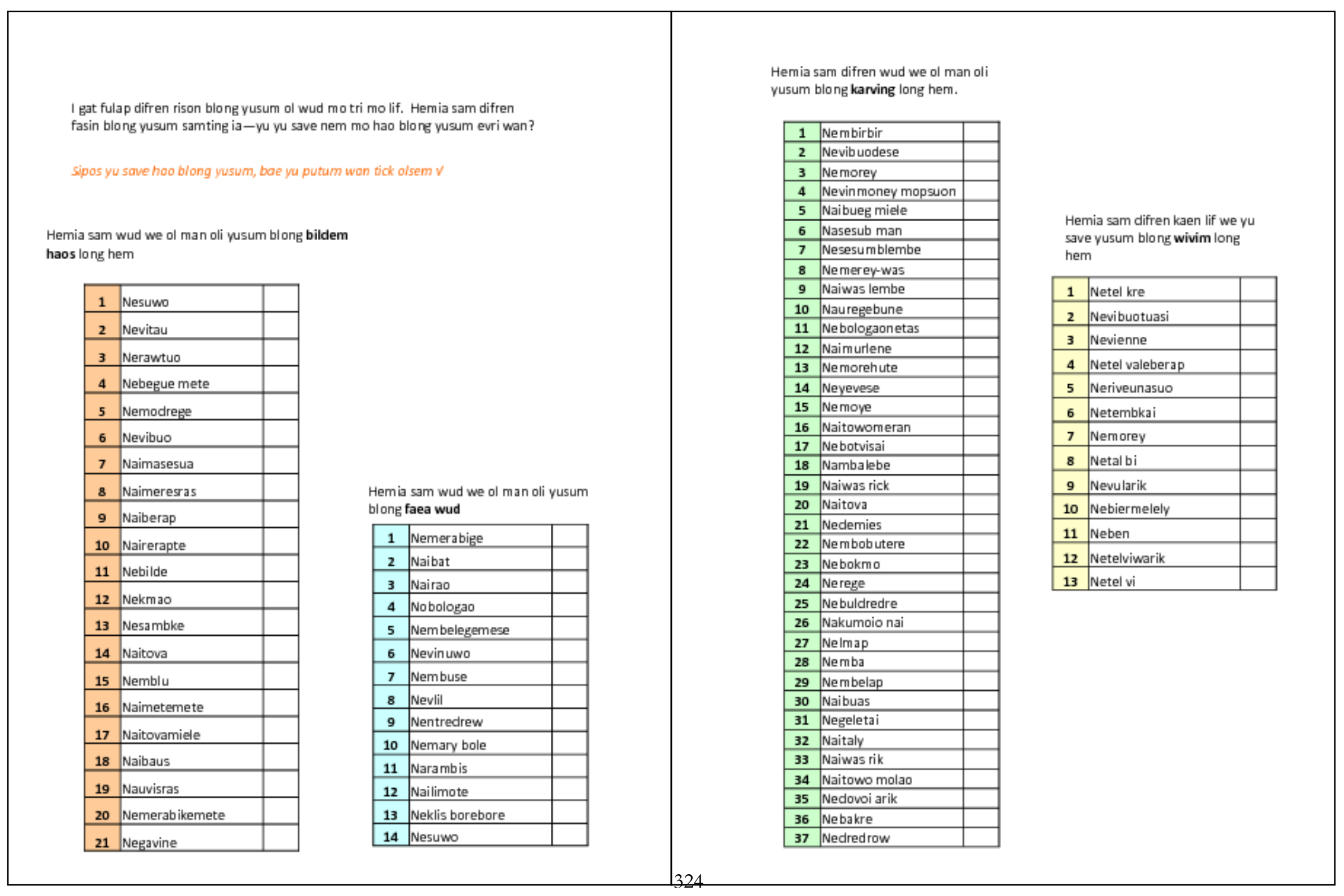


Page Twelve: Information

Buk ia I kaveremap wan smolsmol part nomo long ol kastom save blong Tavend rua. Sipos yu no wantem ol nara samting I lus, yumi mas go faendemaot ol infomesen ia.

Rememba-kastom save hemi laef blong yumi. Sipos I lus, bae I no save kam bak bakagen. I no gat nara ples long world we kastom save I semak olsem Tavendrua - bae yumi mas lukaotem hem ol taem.

ol ol dfela man mo woman long ples ia oli gat fulap save blong telemaot. sipos yu wantem lanem sam lif mo hao nao blong lanem, bae yu jes go askem.

Lanwis hemi stampa long evri kaen kastom save. Saed longol lif, sipos man I no tokto $\mathrm{k} N$ ovol bae hemi no save gud hao nao blong tokbaot ol lif, mo ol nem mo save blong yusum evri wan bae I lus.

Tankyu tumas long ol man mo woman we oli bin givim taem mo waes long ol interviu blong mekem buk io. 


\subsection{Dominant vegetation of Malekula}

Courtesy of the Vanuatu Department of Lands and Survey

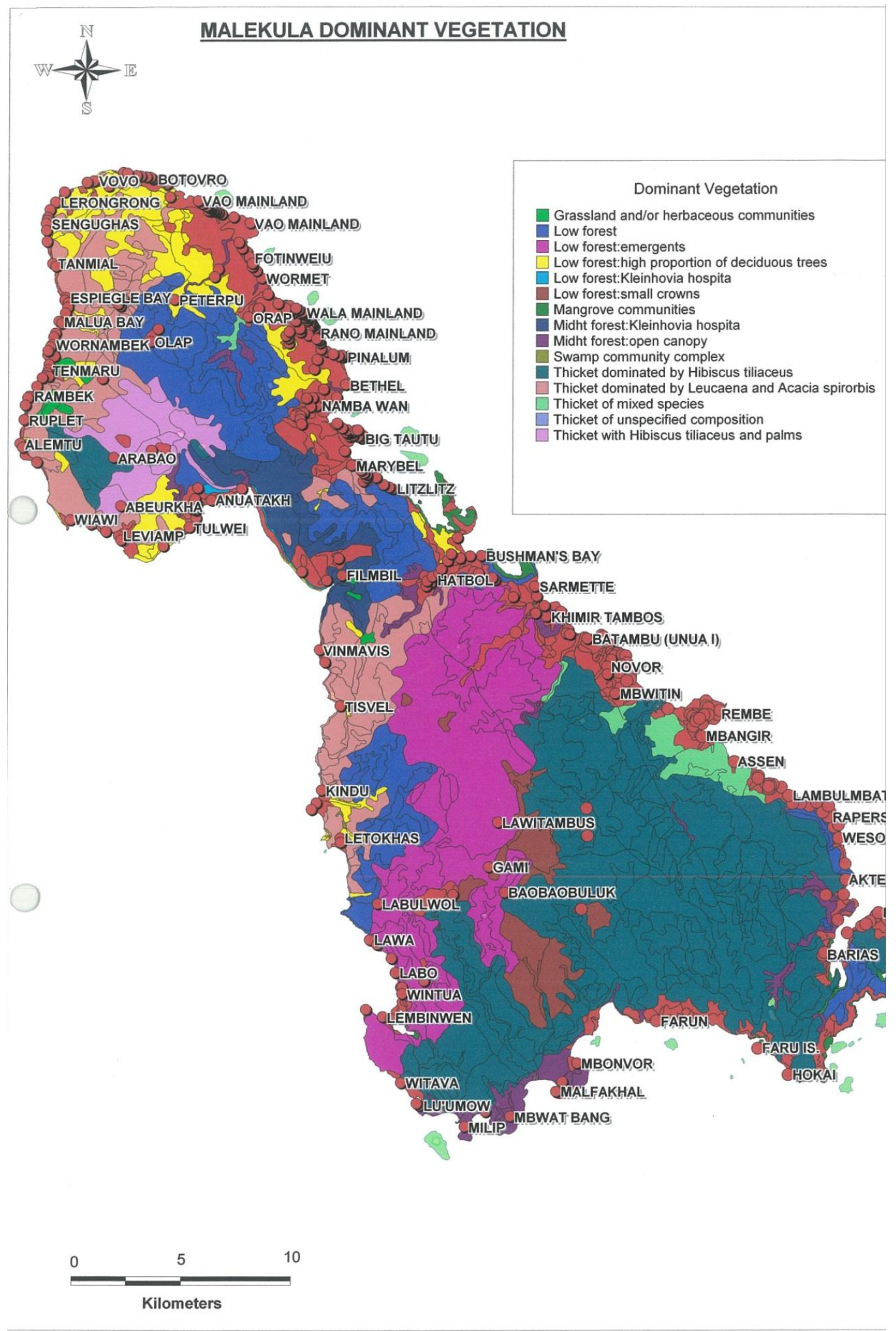

